ESTIMATION OF DETERMINISTIC AND STOCHASTIC IMU ERROR PARAMETERS

A THESIS SUBMITTED TO

THE GRADUATE SCHOOL OF NATURAL AND APPLIED SCIENCES OF

MIDDLE EAST TECHNICAL UNIVERSITY

BY

DERYA ÜNSAL

IN PARTIAL FULLFILLMENT OF THE REQUIREMENTS

FOR

THE DEGREE OF MASTER OF SCIENCE

IN

ELECTRICAL AND ELECTRONICS ENGINEERING

FEBRUARY 2012 
Approval of the thesis:

\section{ESTIMATION OF DETERMINISTIC AND STOCHASTIC IMU ERROR PARAMETERS}

submitted by DERYA UNSAL in partial fulfillment of the requirements for the degree of Master of Science in Electrical and Electronics Engineering Department, Middle East Technical University by,

Prof. Dr. Canan Özgen

Dean, Graduate School of Natural and Applied Sciences

Prof. Dr. İsmet Erkmen

Head of Department, Electrical and Electronics Engineering

Prof. Dr. Kerim Demirbaş

Supervisor, Electrical and Electronics Engineering Dept., METU

\section{Examining Committee Members}

Prof. Dr. Tolga Çiloğlu

Electrical and Electronics Engineering Dept., METU

Prof. Dr. Kerim Demirbaş

Electrical and Electronics Engineering Dept., METU

Assoc. Prof. Dr. Çağatay Candan

Electrical and Electronics Engineering Dept., METU

Assist. Prof. Dr.Mustafa Doğan

Control Engineering Dept., Doğuş University

M.Sc.Uğur Kayasal

Roketsan Missiles Industries Inc.

Date:

$\underline{03.02 .2012}$ 
I hereby declare that all information in this document has been obtained and presented in accordance with academic rules and ethical conduct. I also declare that, as required by these rules and conduct, $I$ have fully cited and referenced all material and results that are not original to this work.

Name, Last name : Derya ÜNSAL

Signature 


\author{
ABSTRACT \\ ESTIMATION OF DERTERMINISTIC AND IMU (INERTIAL MEASUREMENT \\ UNIT) ERROR PARAMETERS \\ Ünsal, Derya \\ M.Sc., Department of Electrical and Electronics Engineering \\ Supervisor: Prof Dr. Kerim Demirbaş
}

February 2012, 122 Pages

Inertial Measurement Units, the main component of a navigation system, are used in several systems today. IMU's main components, gyroscopes and accelerometers, can be produced at a lower cost and higher quantity. Together with the decrease in the production cost of sensors it is observed that the performances of these sensors are getting worse. In order to improve the performance of an IMU, the error compensation algorithms came into question and several algorithms have been designed. Inertial sensors contain two main types of errors which are deterministic errors like scale factor, bias, misalignment and stochastic errors such as bias instability and scale factor instability. Deterministic errors are the main part of error compensation algorithms. This thesis study explains the methodology of how the deterministic errors are defined by 27 state static and 60 state dynamic rate table calibration test data and how those errors are used in the error compensation model. In addition, the stochastic error parameters, gyroscope and bias instability, are also modeled with Gauss Markov Model and instant sensor bias instability values are estimated by Kalman Filter algorithm. Therefore, accelerometer and gyroscope bias instability can be compensated in real time. In conclusion, this thesis study explores how the IMU performance is improved by compensating the deterministic end stochastic errors. The simulation results are supported by a real IMU test data. 
Keywords: Inertial Measurement Unit, gyroscope, accelerometer, Kalman filter. 


\title{
ÖZ
}

\section{AÖB (ATALETSEL ÖLÇÜM BİRIMİ) DETERMINISTIIK VE OLASILIKSAL HATA PARAMETRELERINIIN KESTIRIMMI}

\author{
Ünsal, Derya \\ Yüksek Lisans, Elektrik ve Elektronik Mühendisliği Bölümü \\ Tez Yöneticisi: Prof. Dr. Kerim Demirbaş
}

Şubat 2012, 122 Sayfa

Seyrüsefer sistemlerinin temel bileşeni olan ataletsel ölçüm birimleri günümüzde bir çok değişik sistemde kullanılmaktadır. Ataletsel ölçüm biriminin ana bileşenleri olan ivmeölçerler ve dönüölçerler üretimdeki maliyetin düşmesi ile birlikte üretim sayılarında artış meydana gelmiştir. İvmeölçer ve dönüölçerlerin düşük maliyetle yüksek sayıda üretilmesi ile birlikte sensör performanslarında düşüş olduğu gözlemlenmektdir. Bu nedenle, ataletsel ölçüm birimlerinin performansını arrtıkmak amacıyla hata telafi algoritmaları gündeme gelmiş ve çok çeşitli algoritmalar tasarlanmıştır. Ataletsel sensörler iki türlü hata içerirler. Bunlar orantı katsayısı hatası, sabit kayma ve eksenel kaçıklık gibi deterministik hatalar ve sabit kayma hatası kararsızlığ1, orantı katsayısı hatası kararsızlığı gibi olasılıksal hatalardır. Deterministik hatalar hata telafi algoritmalarının temel parçalarını oluşturmaktadır. Bununla birlikte uzun süreli seyrüsefer sistemi kullanımlarında olasılıksal hatalar kritik önem taşımaktadır. Bu tez çalışması, dönü tablası kullanılarak gerçekleştirilen 27 durumlu statik pozisyon testi ve 60 durumlu dinamik kalibrasyon testi verilerinden deterministik hatların nasıl hesaplandığını ve belirlenen hataların hata telafi modeli içerisinde nasıl kullanıldığını anlatmaktadır. Ayrıca, olasılıksal hata parametrelerinin Gauss-Markov süreci ile modellenerek sensörlerin sabit hata 
kararsızlıklarının anlık olarak kestirimi Kalman süzgeci algoritması kullanılarak gerçekleştirilmiştir. İvmeölçerler ve dönüölçerlerin sabit hata kararsızlıklarının anlık olarak kestirilmesi ile birlikte ataletsel ölçüm biriminin olasılıksal hataları gerçek zamanlı olarak telafi edilebilecektir. Sonuç olarak, bu tez çalışmasında deterministik ve olasılıksal sensör hatalarının telafisi yapılarak ataletsel ölçüm birimi performansının nasıl arttırıldığı araştırılmıştır.

Anahtar Kelimeler: Ataletsel ölçüm birimi, dönüölçer, ivmeölçer, Kalman süzgeci. 
To My Family

viii 


\section{ACKNOWLEDGEMENTS}

I would like to express my sincere thanks to my supervisor Prof. Dr. Kerim Demirbaş for his guidance, advice and support throughout this thesis.

I am also thankful to my old advisor Assist. Prof. Dr. Mustafa Doğan for his endless support and encouragement.

I also wish to thank to my colleagues at ROKETSAN Inc. for their support, encouragements and friendship.

I would like to express my special thanks to my family for their love, endless support and patience.

Finally, the support provided by ROKETSAN Inc. to this thesis is greatly acknowledged. 


\section{TABLE OF CONTENTS}

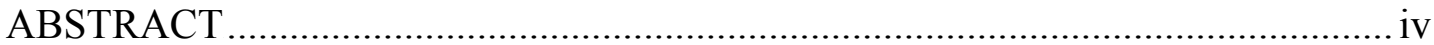

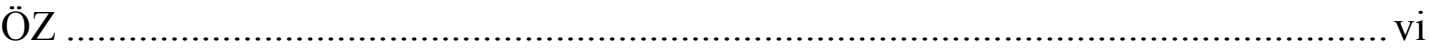

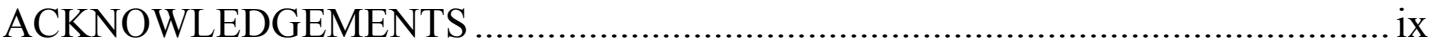

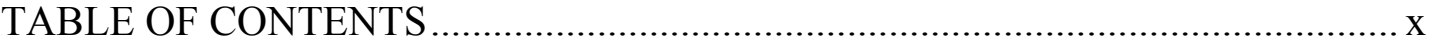

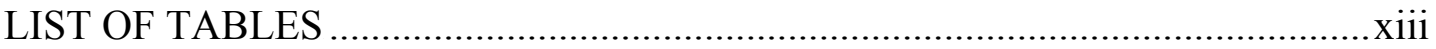

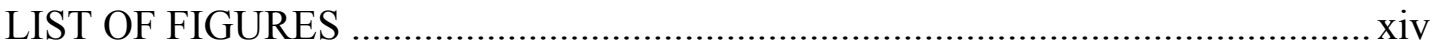

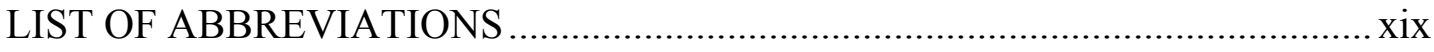

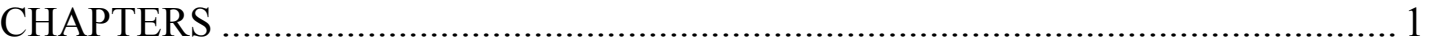

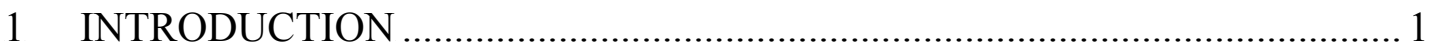

2 THEORETICAL BACKGROUND ABOUT IMU …..................................... 4

2.1 Inertial Measurement Unit................................................................... 4

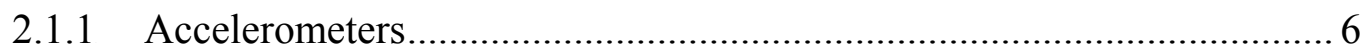

2.1.1.1 MEMS Accelerometers ........................................................... 8

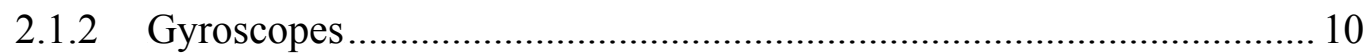

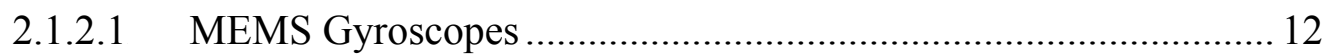

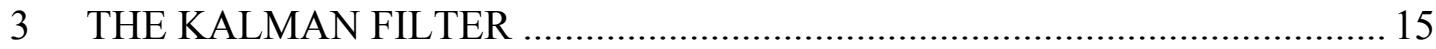

3.1 Properties of the Kalman Filter .............................................................. 15

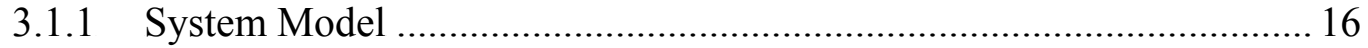

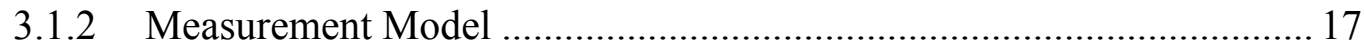

3.2 The Mathematical Model of The Kalman Filter........................................ 18

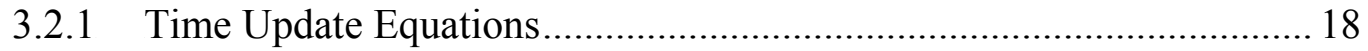

3.2.2 Measurement Update Equations …................................................. 19 


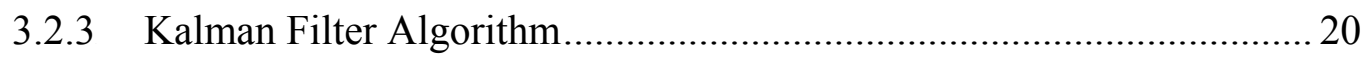

3.3 The Role of the Kalman Filter In This Thesis Study................................ 22

4 SENSOR ERROR PARAMETERS AND IMU ERROR MODELS ................. 24

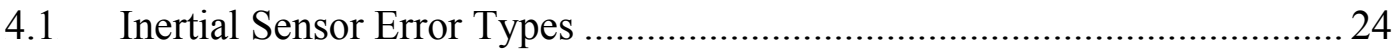

4.1.1 Deterministic Error Parameters of Accelerometers ............................ 25

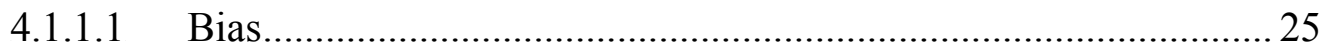

4.1.1.2 Scale Factor Error ............................................................ 26

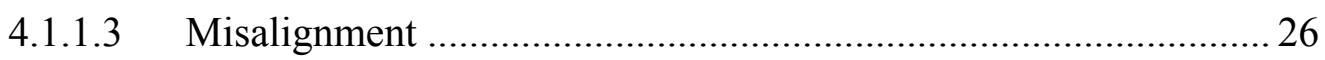

4.1.2 Deterministic Error Parameters of Gyroscopes................................... 27

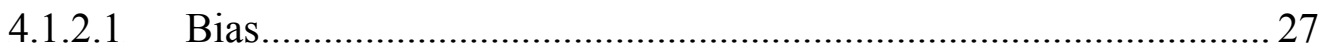

4.1.2.2 Scale Factor Error …........................................................... 28

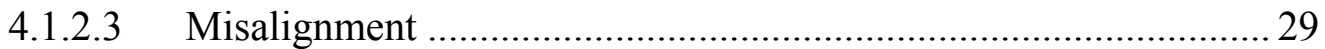

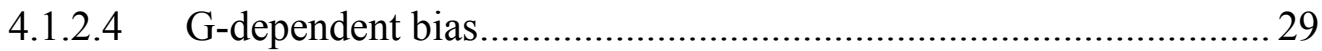

4.1.3 Stochastic Error Parameters of Accelerometers and Gyroscopes .......... 31

4.1.3.1 Bias Instability (Bias Drift) ..................................................... 31

4.1.3.2 Scale Factor Instability (Scale Factor Drift) ............................... 32

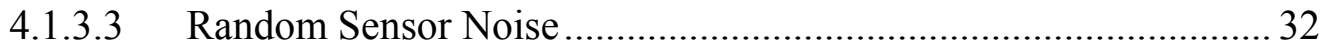

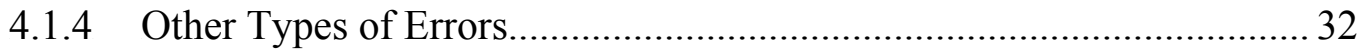

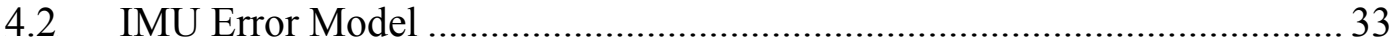

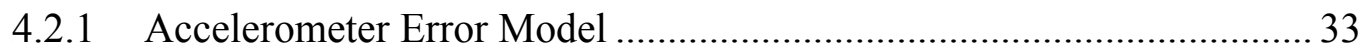

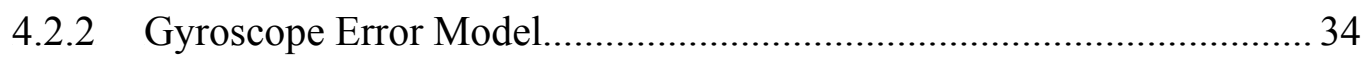

5 ESTIMATION OF DETERMINISTIC ERROR PARAMETERS .................... 36

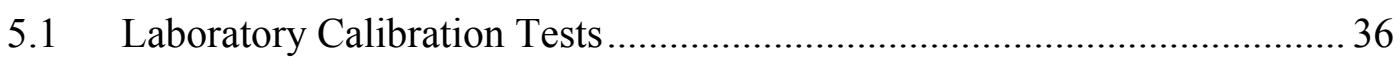

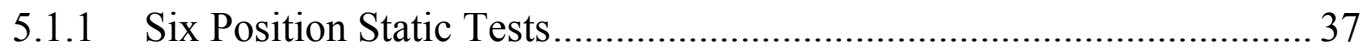

5.1.1.1 Data Processing Methods for Six Position Test............................ 38

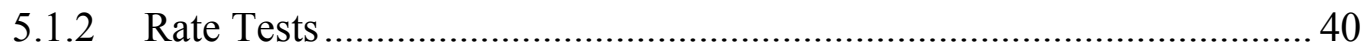




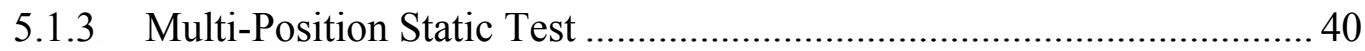

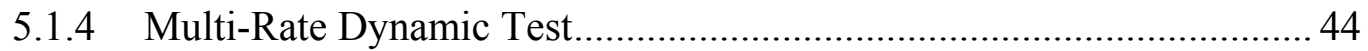

5.1.5 Data Processing Algorithm for Multi-Position Static and Multi-Rate

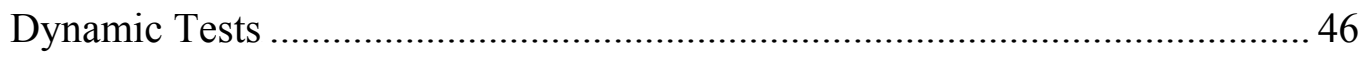

5.2 Deterministic Error Compensation Model ..................................................5 57

6 ESTIMATION OF STOCHASTIC ERROR PARAMETERS ......................... 59

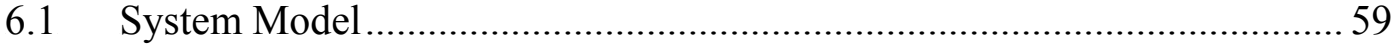

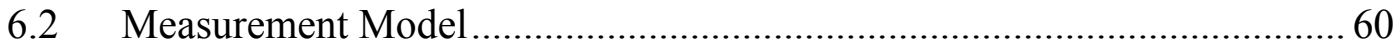

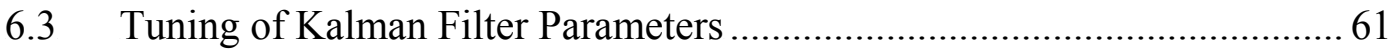

6.3.1 Error Covariance Matrix Initialization................................................. 61

6.3.2 Tuning of Measurement and Process Noise Covariance Matrix............ 62

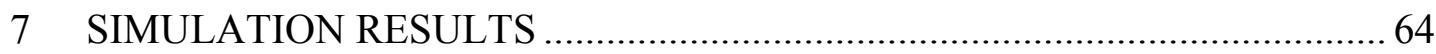

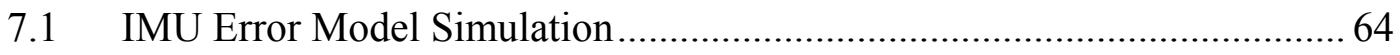

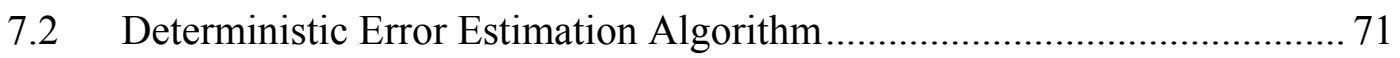

7.3 IMU Error Compensation (Calibration) Model Simulation ........................ 76

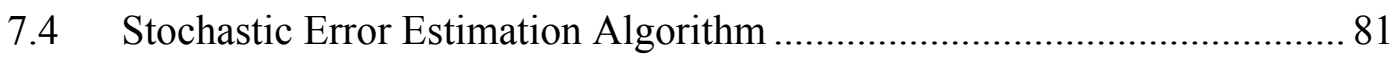

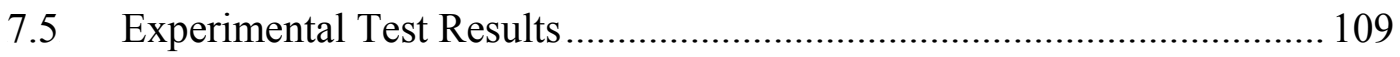

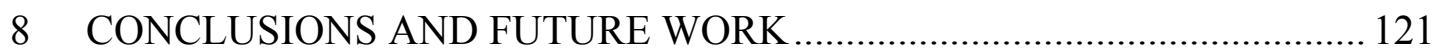

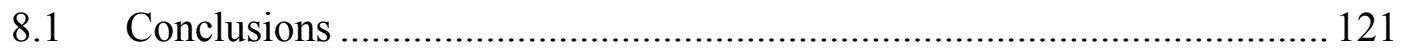

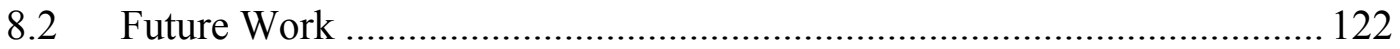

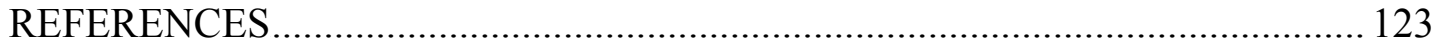

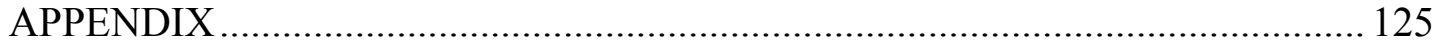

FLOWCHART OF THE SIMULATION AND EXPERIMENTAL STUDIES ..... 125 


\section{LIST OF TABLES}

\section{TABLES}

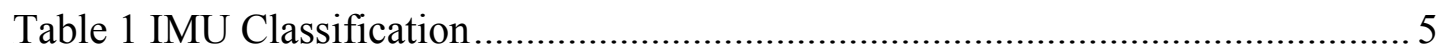

Table 2 System Model Parameters................................................................... 17

Table 3 Measurement Model Parameters................................................................ 18

Table 4 Time Update Equations Parameter Definitions ........................................... 19

Table 5 Measurement Update Equations Parameter Definitions .............................. 20

Table 6 Simulated IMU Deterministic Error Parameters......................................... 65

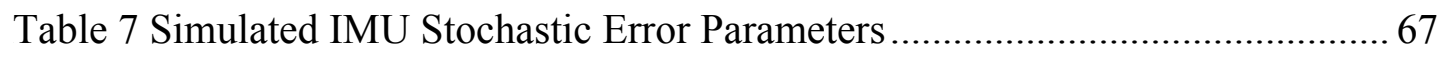

Table 8 Deterministic Error Estimation Algorithm Outputs (Gyroscope)................. 71

Table 9 Deterministic Error Estimation Algorithm Outputs (Accelerometer)........... 72

Table 10 Six position direct method results (gyroscopes) …................................ 76

Table 11 Six position direct method results (accelerometers) ............................... 76

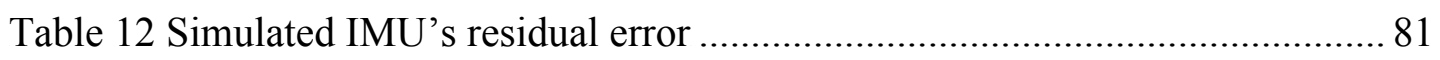

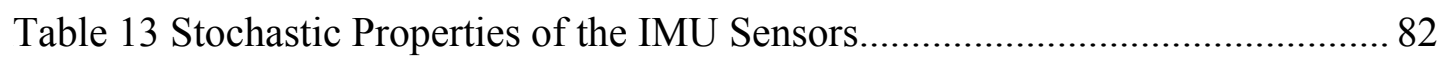

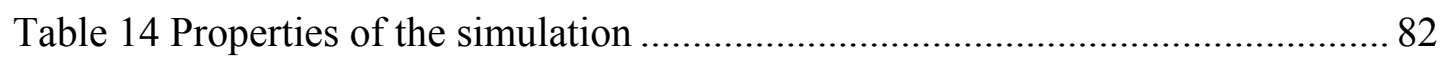

Table 13Deterministic Error Estimation Results (Real data)............................... 110

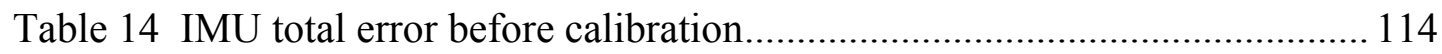

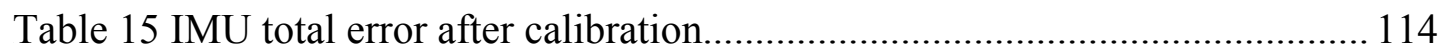




\section{LIST OF FIGURES}

\section{FIGURES}

Figure 1 Gimballed and Strapdown Accelerometers 5

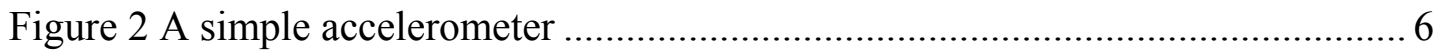

Figure 3 Current Accelerometer Technology Applications .................................... 7

Figure 4 Near-term accelerometer technology applications .................................... 8

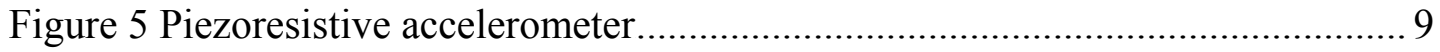

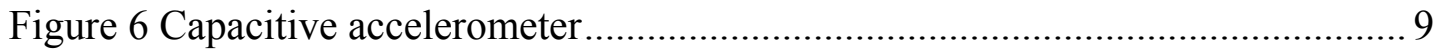

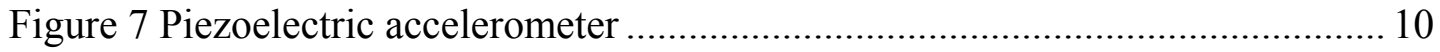

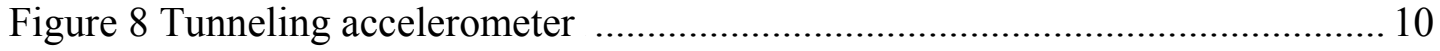

Figure 9 Current gyro technology applications................................................. 11

Figure 10 Near-term gyro technology applications ............................................ 12

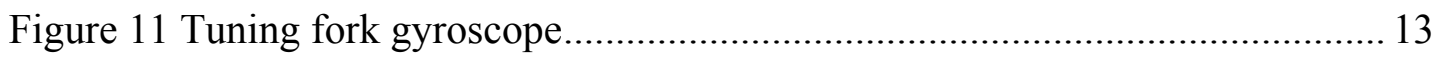

Figure 12 Vibrating wheel gyroscope .................................................................. 13

Figure 13 Ring Laser and Fiber Optic Gyroscope ............................................... 14

Figure 14 System and Measurement Model Implementation .................................. 18

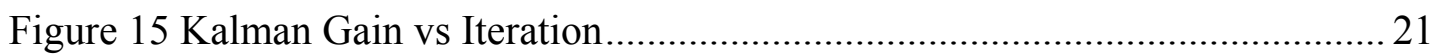

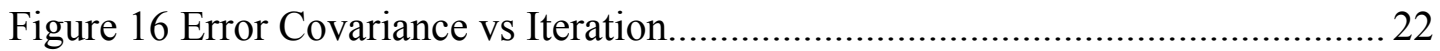

Figure 17 Recursive structure of the Kalman filter................................................ 22

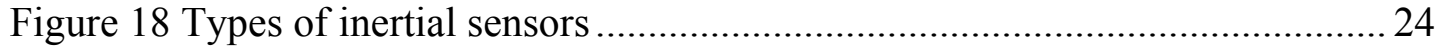

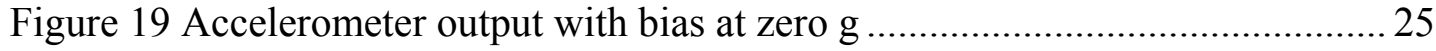

Figure 20 Accelerometer output without bias at zero g ..........................................2 26

Figure 21 Gyro output with bias at zero rotational input ........................................2 27

Figure 22 Gyro output without bias at zero rotational input .................................. 28

xiv 
Figure 23 Gyro output with bias and scale factor error at $200 \mathrm{deg} / \mathrm{sn}$

Figure 24 Gyro output without bias and scale factor error at $200 \mathrm{deg} / \mathrm{sn}$................. 29

Figure 25 Representation of Scale Factor and Bias ............................................. 30

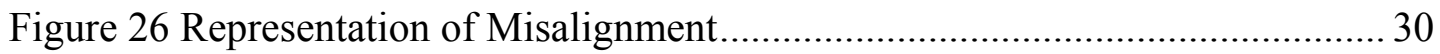

Figure 27 Simulated IMU measurement axis configuration ................................... 41

Figure 28 Multi-Position Test Configurations …............................................... 41

Figure 29 Multi Position Test Procedure …....................................................... 42

Figure 30 Multi-position static test $\mathrm{x}$ acc output................................................. 43

Figure 31 Multi-position static test y acc output.................................................... 43

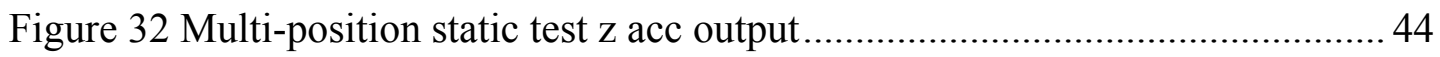

Figure 33 Multi-rate dynamic test $x$ gyro output ................................................. 45

Figure 34 Multi-rate dynamic test y gyro output ............................................... 45

Figure 35 Multi-rate dynamic test $\mathrm{z}$ gyro output ................................................... 46

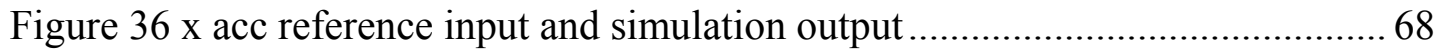

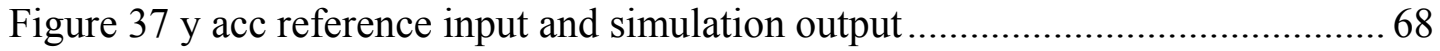

Figure $38 \mathrm{z}$ acc reference input and simulation output .........................................6 69

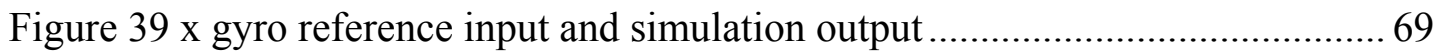

Figure $40 \mathrm{y}$ gyro reference input and simulation output ........................................ 70

Figure $41 \mathrm{z}$ gyro reference input and simulation output ....................................... 70

Figure $42 \times$ gyro reference input and simulation output (six position test) ...............73

Figure 43 y gyro reference input and simulation output (six position test) .............. 73

Figure $44 \mathrm{z}$ gyro reference input and simulation output (six position test) .............. 74

Figure $45 \mathrm{x}$ acc reference input and simulation output (six position test) ................ 74

Figure 46 y acc reference input and simulation output (six position test) ................ 75

Figure $47 \mathrm{z}$ acc reference input and simulation output (six position test) ................ 75

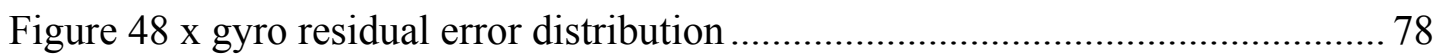




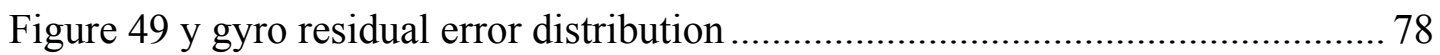

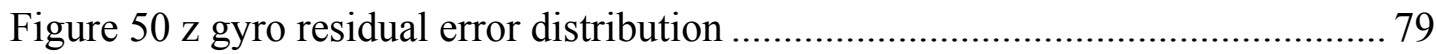

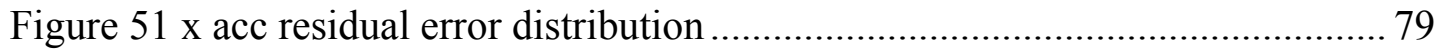

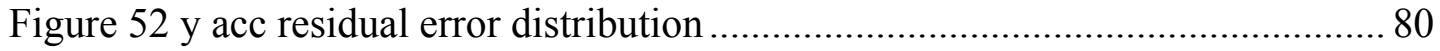

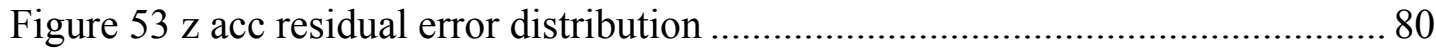

Figure 54 Gyroscope bias instability estimation Kalman gain variation .................. 84

Figure 55 Accelerometer bias instability estimation Kalman gain variation............. 85

Figure 56 Gyroscope bias instability estimation error covariance variation............... 85

Figure 57 Accelerometer bias instability estimation error covariance variation .......86 86

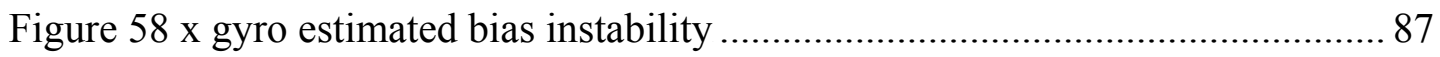

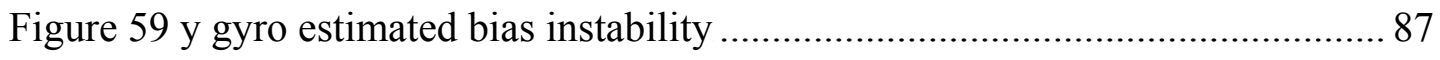

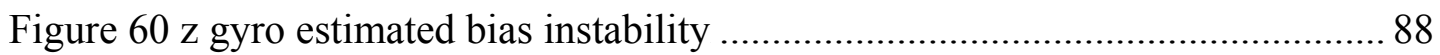

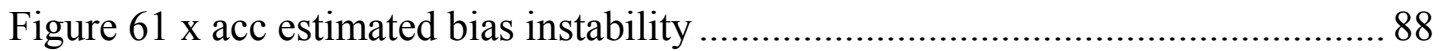

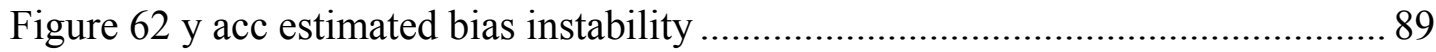

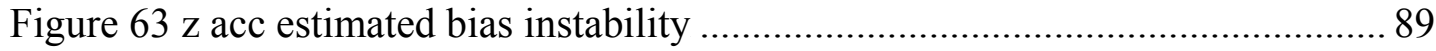

Figure 64 Gyroscpe bias instability estimation Kalman gain variation ( $\mathrm{R}$ effect)..... 91

Figure 65 Accelerometer bias instability estimation Kalman gain variation ( $\mathrm{R}$ effect)

Figure 66 Gyroscope bias instability estimation error covariance variation ( $\mathrm{R}$ effect)

Figure 67 Accelerometer bias instability estimation error covariance variation ( $R$

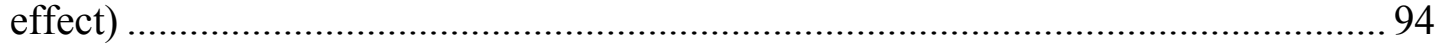

Figure $68 \mathrm{x}$ gyro estimated bias instability $(\mathrm{R}$ effect) .............................................. 95

Figure 69 y gyro estimated bias instability (R effect) ............................................95

Figure $70 \mathrm{z}$ gyro estimated bias instability (R effect) ........................................... 96

Figure $71 \mathrm{x}$ acc estimated bias instability ( $\mathrm{R}$ effect) ............................................. 96

Figure 72 y acc estimated bias instability ( $\mathrm{R}$ effect) ............................................ 97 
Figure $73 \mathrm{z}$ acc estimated bias instability (R effect)

Figure 74 Gyroscope bias instability estimation Kalman gain variation (correlation time effect)

Figure 75 Accelerometer bias instability estimation Kalman gain variation (correlation time effect).

Figure 76 Gyroscope bias instability estimation error covariance variation (correlation time effect).

Figure 77 Accelerometer bias instability estimation error covariance variation (correlation time effect).

Figure $78 \mathrm{x}$ gyro estimated bias instability (correlation time effect ).........

Figure 79 y gyro estimated bias instability (correlation time effect )..... 104

Figure $80 \mathrm{z}$ gyro estimated bias instability (correlation time effect) 105

Figure $81 \mathrm{x}$ acc estimated bias instability (correlation time effect) 106

Figure 82 y acc estimated bias instability (correlation time effect) 107

Figure $83 \mathrm{z}$ acc estimated bias instability (correlation time effect ) 108

Figure 84 Representation of MEMS IMU 109

Figure 85 Experimental test setup 109

Figure 86 uncalibrated and calibrated $\mathrm{x}$ acc output (real data)

Figure 87 uncalibrated and calibrated y acc output (real data)

Figure 88 uncalibrated and calibrated $\mathrm{z}$ acc output (real data)

Figure 89 uncalibrated and calibrated x gyro output (real data)

Figure 90 uncalibrated and calibrated y gyro output (real data)

Figure 91 uncalibrated and calibrated $\mathrm{z}$ gyro output (real data)

Figure 92 Gyroscope bias instability estimation Kalman gain variation (real data) 115

Figure 93 Accelerometer bias instability estimation Kalman gain variation (real data)

Figure 94 Gyroscope bias instability estimation error covariance variation (real data) 116 
Figure 95 Accelerometer bias instability estimation error covariance variation (real

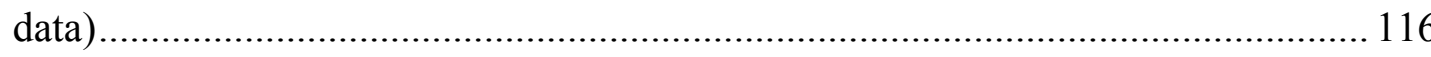

Figure 96 x gyro estimated bias instability (real data) ....................................... 117

Figure 97 y gyro estimated bias instability (real data) ........................................ 117

Figure $98 \mathrm{z}$ gyro estimated bias instability (real data) ......................................... 118

Figure $99 \mathrm{x}$ acc estimated bias instability (real data) .......................................... 118

Figure $100 \mathrm{y}$ acc estimated bias instability (real data).......................................... 119

Figure $101 \mathrm{z}$ acc estimated bias instability (real data) .......................................... 119 


\section{LIST OF ABBREVIATIONS}

ACC : ACCELEROMETER

FMS : FLIGHT MOTION SIMULATOR

GPS : GLOBAL POSITIONING SYSTEM

GYRO : GYROSCOPE

IMU : INERTIAL MEASUREMENT UNIT

INS: INERTIAL NAVIGATION SYSTEM

KF: KALMAN FILTER

LSF: LEAST SQUARES FITTING

RMS : ROOT MEAN SQUARE 


\section{CHAPTER 1}

\section{INTRODUCTION}

Navigation is the art of getting from one place to another, safely and efficiently [1]. From past to present, several tools and systems such as compasses, maps, sun, stars were used for navigation. In today's world these tools have been replaced by electronic equipments such as sensors, antennas, etc. These electronic equipments form the basis of the modern navigation systems. Inertial Navigation Systems and Global Positioning Systems can be shown as an example for the modern navigation systems. Nowadays several types of INS, GPS and integrated INS/GPS are used in different platforms such as aircrafts, ships, guided missiles and UAVs.

GPS acquire and process satellite signals to calculate navigation parameters such as position, velocity and attitude, according to the received signals. GPS always need satellite signals and this is the major drawback of GPS. However, INS use IMU outputs to construct position velocity and attitude by processing the navigation equations. Therefore IMUs are the major part of inertial navigation systems.

An inertial measurement unit is a device, which is used to measure linear acceleration and angular rate. Inertial measurement units contain two types of sensor, accelerometer and gyroscope. An accelerometer measures linear acceleration about its sensitivity axis and integrated acceleration measurements are used to calculate velocity and position. Besides a gyroscope measures angular rate about its sensitivity axis and gyroscope outputs are used to maintain orientation in space. In addition to these sensors IMU has a processor. IMU calibration algorithm runs on the processor and also the communication interface in the processor. 
Inertial measurement units are divided into various classes such as strategic, navigation, tactical, industrial and automotive grade. These classes are determined according to performance levels of sensors which are used in IMUs. The navigation grade IMUs are the highest grade systems and they are used on ships, ballistic missiles and aircrafts.

The cost of an IMU increases when the sensor performance requirements increase. The major reasons for the cost increase can be explained in two ways. The first reason is the highly skilled production line requirement and the second reason is the decrease in the percentage of utilizable sensor in the batch. Therefore, in order to improve the performance of inertial sensors, the calibration algorithms and the error compensation models were researched and developed. Thereby both low-cost and high-performance IMUs could be produced.

The main objective of this thesis work is to develop methods in order to estimate deterministic and stochastic error parameters of MEMS based inertial measurement units. Additionally, improving the performance of IMUs is aimed by using these estimated parameters. Therefore an error calibration algorithm is implemented and estimated parameters are used in this algorithm.

In this thesis, an error model which includes both deterministic and stochastic errors is constituted to simulate the behavior of a MEMS-based IMU. Outputs of the error model simulation are used as input for deterministic error estimation algorithm. After the estimation of deterministic error parameters, these parameters are embedded into the error compensation simulation to compensate the effects of deterministic errors. Also, the Kalman filter algorithm is developed according to the stochastic error model and used for estimating stochastic errors. After the determination of stochastic errors, outputs of the error compensation algorithm are corrected by subtracting estimated stochastic errors

This thesis study is composed of eight chapters . 
Chapter 1 provides introduction and summary about content of the chapters.

The second chapter of this thesis gives a theoretical background about structure of IMUs, types and production methods of inertial sensors.

In Chapter 3, the Kalman filter algorithm and its properties are presented. Additionally mathematical model of the Kalman filter is given. The role of the Kalman filter is also explained.

Chapter 4 gives general information about inertial sensor error types, error models and provides equations of the sensor outputs. Deterministic and stochastic error types are explained separately. Besides, the IMU error model is described in detail.

The fifth chapter of this thesis explains deterministic error estimation techniques. IMU calibration test procedures such as static and dynamic tests are introduced and the technique which is suggested in the scope of this thesis study is explained.

In Chapter 6 stochastic sensor error estimation techniques are explained. System model and measurement model are also provided.

Chapter 7 presents simulation results. These results include both deterministic and stochastic error estimation simulation and algorithm results. In addition, simulation results are evaluated and different simulation results which are carried under different conditions are discussed.

In Chapter 8 summary of this thesis study and suggestions for future are given 


\section{CHAPTER 2}

\section{THEORETICAL BACKGROUND ABOUT IMU}

Inertial measurement units typically contain three orthogonal gyroscopes a and three orthogonal accelerometers, measuring angular velocity and linear acceleration [2]. As mentioned in the previous chapter, inertial measurement units are divided into various classes and these classes are determined according to error limits of inertial sensors such as scale factor, bias error and sensor noise. These sensor limits vary depending on the types of sensors.

This chapter gives theoretical background about IMUs and inertial sensor technologies. Section 2.1 summarizes the properties of an IMU an Section 2.1.1 gives some information about accelerometers and their features. Similar to the Section 2.1.1, Section 2.1.2 discusses types of gyroscopes and their features.

\subsection{Inertial Measurement Unit}

The combination of gyros, accelerometers, and supporting structure assembly and electronics was referred to as the IMU.. There are two types of inertial measurement configuration: gimballed or stabilized platform, strapdown or analytical platform. The primary difference between the gimballed and strapdown system is the environment in which the accelerometers and gyroscopes must function. Gimballed IMUs, first version of IMU configurations, consist of a platform isolated from vehicle rotations by gimbals. Since the platform does not rotate with the vehicle, its orientation remains fixed. On the other hand, strapdown IMUs are attached rigidly to 
the body of the vehicle and IMUs move with the vehicle. Gimballed IMUs provide very accurate navigation data but mechanical complexities and costs of the gimballed IMUs are very high. Strapdown IMUs are mechanically simple, lower cost, more useful due to small size. Therefore, strapdown inertial measurement units are preferred today $[3,4,5]$.

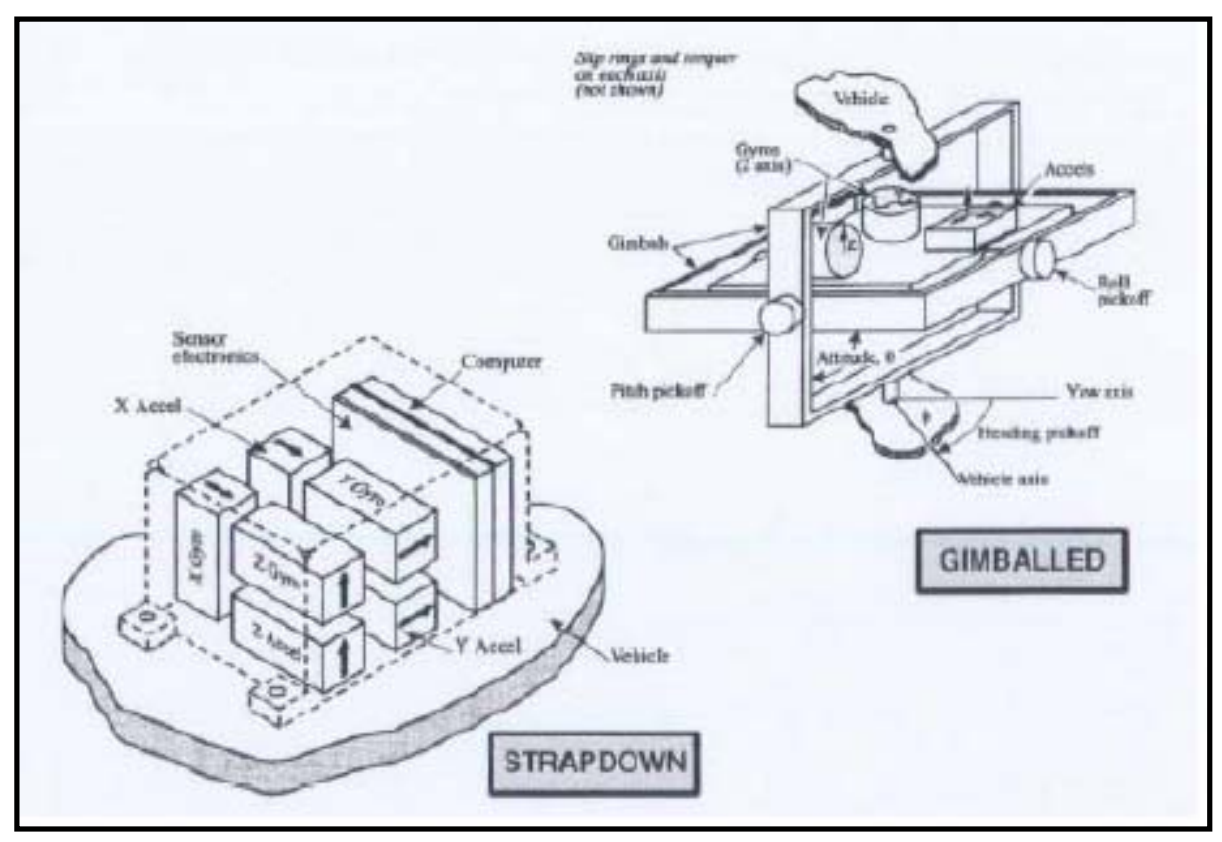

Figure 1 Gimballed and Strapdown Accelerometers

The major disadvantage of the strapdown inertial system is increase in computational complexity. However, recent advances in computer and sensor technology allow to overcome this problem [3].

As mentioned Chapter 1, according to their error performances, IMUs can be classified into different groups. Table-1 presents these groups and their error limitations.

Table 1 IMU Classification[8]

\begin{tabular}{|c|c|c|c|}
\hline Error & $\begin{array}{c}\text { Tactical Grade } \\
\quad<20 \mathrm{~km} / \mathrm{h}\end{array}$ & $\begin{array}{l}\text { Navigation Grade } \\
\quad<1 \mathbf{k m} / \mathbf{h}\end{array}$ & $\begin{array}{c}\text { Strategic Grade } \\
\quad<30 \mathrm{~m} / \mathrm{h}\end{array}$ \\
\hline Gyro Drift Rate & $1-10 \mathrm{deg} / \mathrm{h}$ & $0.015 \mathrm{deg} / \mathrm{h}$ & $0.0001 \mathrm{deg} / \mathrm{h}$ \\
\hline Acc Bias & $100-1000 \mu \mathrm{g}$ & $50-100 \mu g$ & $1 \mu \mathrm{g}$ \\
\hline Cost of IMU & $<10000 \$$ & $10000-70000 \$$ & $>200000 \$$ \\
\hline
\end{tabular}


The following sections explain the basic parts of inertial measurement units, accelerometers and gyroscopes.

\subsubsection{Accelerometers}

An accelerometer measures the acceleration in an inertial reference frame, which can be used to estimate the acceleration of moving body [6]. The integrated value of an accelerometer gives the velocity and second integration gives the distance travelled. These calculated parameters are required for inertial navigation.[7]

The main components of a typical accelerometer are a proof mass, a suspension to hold the mass, and a pickoff, which relates an output signal to the induced acceleration [6]

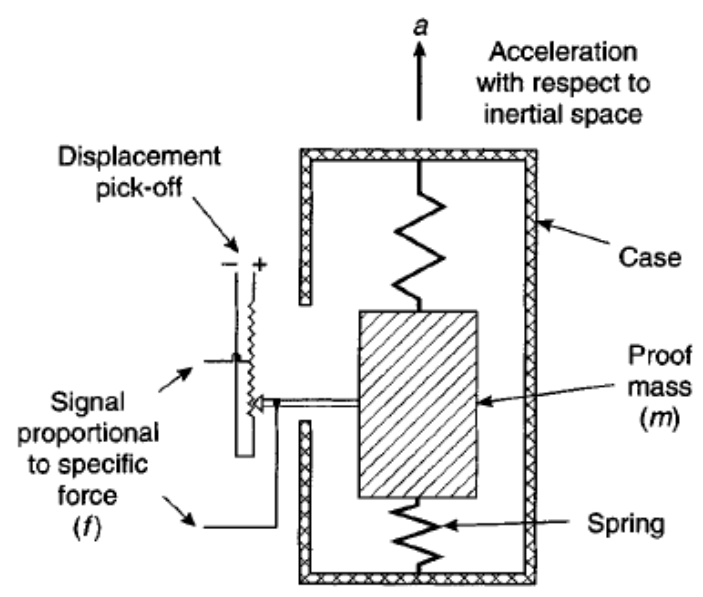

Figure 2 A simple accelerometer (Figure is taken from [3])

When the accelerometer is subjected to an acceleration along its sensitive axis, the proof mass tends to resist the change in movement owing to its inertia. As a result, the mass is displaced with respect to the body. The force acting on the mass will be balanced by the tension in the spring and the net extension gives a measure of the applied force[3].

$$
F=m a=m f+m g
$$


$F$ is the total force, $m$ is the mass, $g$ is the gravitational acceleration and $f$ is the specific force. An accelerometer can not measure gravitational force. Therefore, specific force $f$ gives the acceleration which is exerted on the sensor.

There are different types of accelerometers are used in different platforms and these types are classified according to production technology. All accelerometers exhibit several errors and these errors depend on the type of accelerometer. Fixed bias, scale factor error, misalignment and random bias can be listed as primary accelerometer errors.

Accelerometers can be divided into 3 main groups. These groups are mechanical accelerometers, quartz accelerometers and MEMS accelerometers. Each group of sensors have different error characteristics. Accordingly, each group has different application areas such as long-range guidance, tactical weapons and commercial applications. Figure 3 shows current accelerometer technology applications.[9]

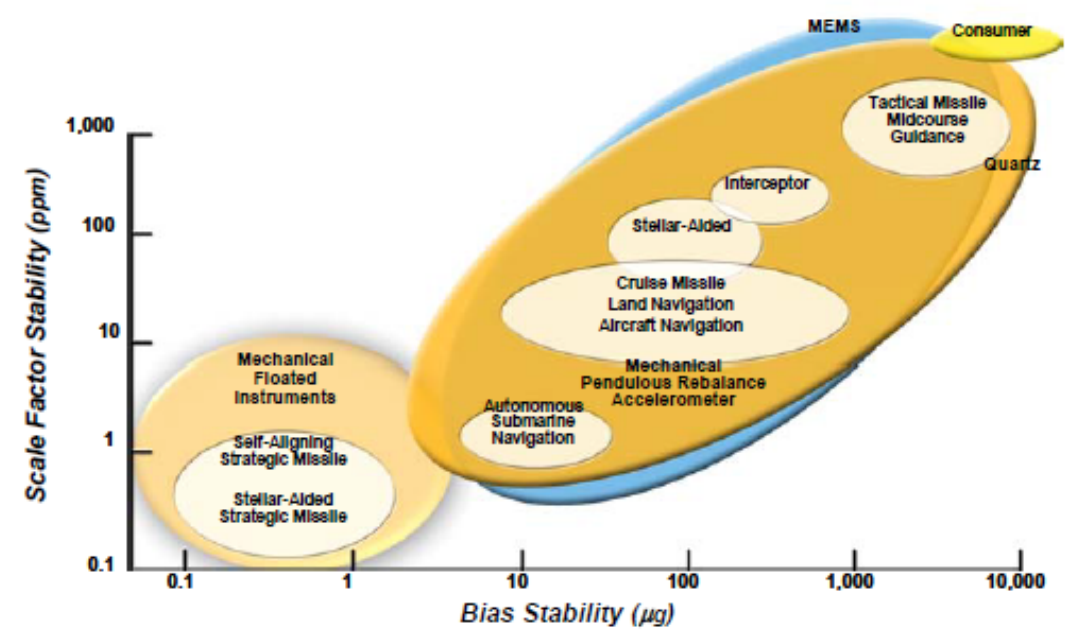

Figure 3 Current Accelerometer Technology Applications (Figure is taken from [9])

As the figure shows current applications are dominated by electromechanical sensors and quartz resonators [9]. Figure 4 presents that the tactical performance end of the accelerometer application spectrum will be dominated by micromechanical 
gyroscopes and higher performance applications will continue to use mechanical and quartz accelerometers.

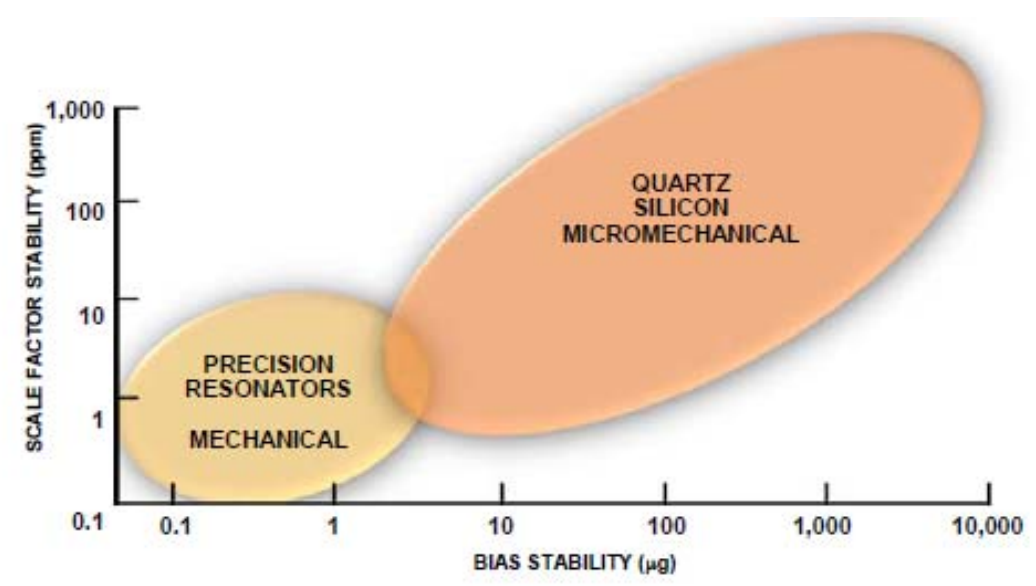

Figure 4 Near-term accelerometer technology applications (Figure is taken from [9])

MEMS accelerometer was used in this thesis study. In this context, MEMS accelerometer error model is given in more detail in Chapter 4. The following section includes structure and working principle of MEMS accelerometers. In addition, other types of accelerometers are also summarized in the following section.

\subsubsection{MEMS Accelerometers}

Types of MEMS accelerometers can be listed as piezoresistive, capacitive, piezoelectric and tunneling accelerometers.

Piezoresistive accelerometers are the first micromachined and commercialized inertial sensors [10]. This type of accelerometers incorporate silicon piezoresistors in their suspension beam. When the frame moves, suspension beams elongate or shorten, which changes the stress and resistivity of embedded piezoresistors The variation in acceleration causes the change in resistance. This change can be evaluated using standard bridge techniques. The simplicity of the structure and fabrication process is the main advantage of piezoresistive accelerometers. On the 
other hand, high temperature sensitivity is the main disadvantage of these sensors. . $[6,11]$

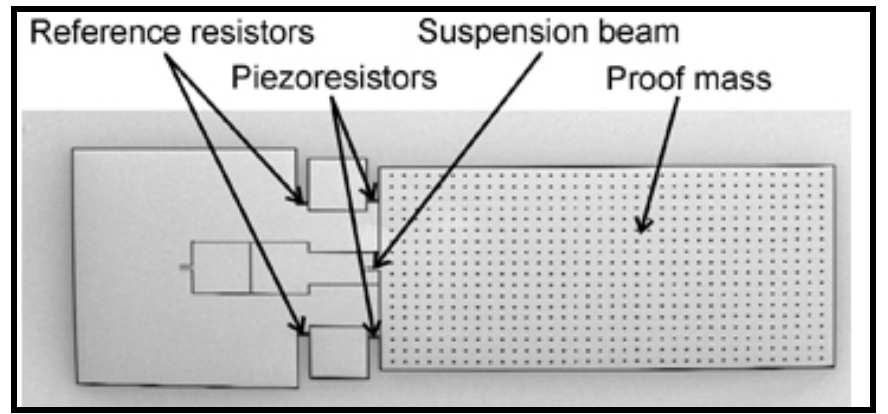

Figure 5 Piezoresistive accelerometer ( Figure is taken from [11])

Capacitive accelerometers use air damped, opposed-plate capacitor as sensing elements. The support frame of an accelerometer moves from its rest position, when the acceleration is applied to the sensor. This movement creates the change in the capacitance between the proof mass and fixed electrodes. In contrast to the piezoresistive accelerometers, temperature sensitivity of the capacitive accelerometers is low $[6,11]$.

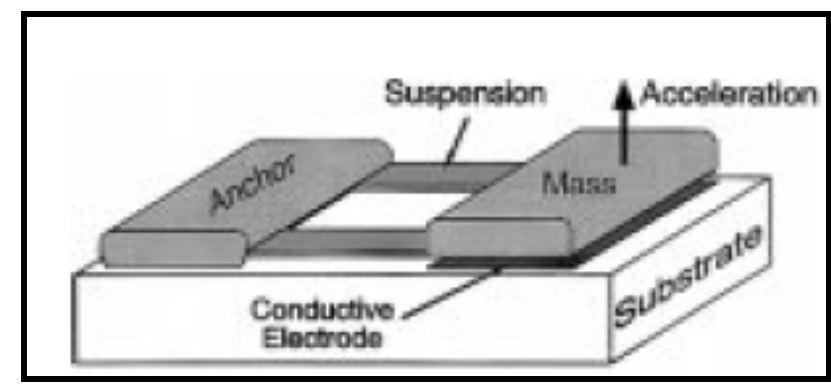

Figure 6 Capacitive accelerometer ( Figure is taken from [11])

Piezorelectric accelerometer use piezoelectric material to sense applied acceleration. Piezoelectric material converts the measured acceleration to electrical signal. Therefore, sensor structure does not need any conversion electronics [6]. 


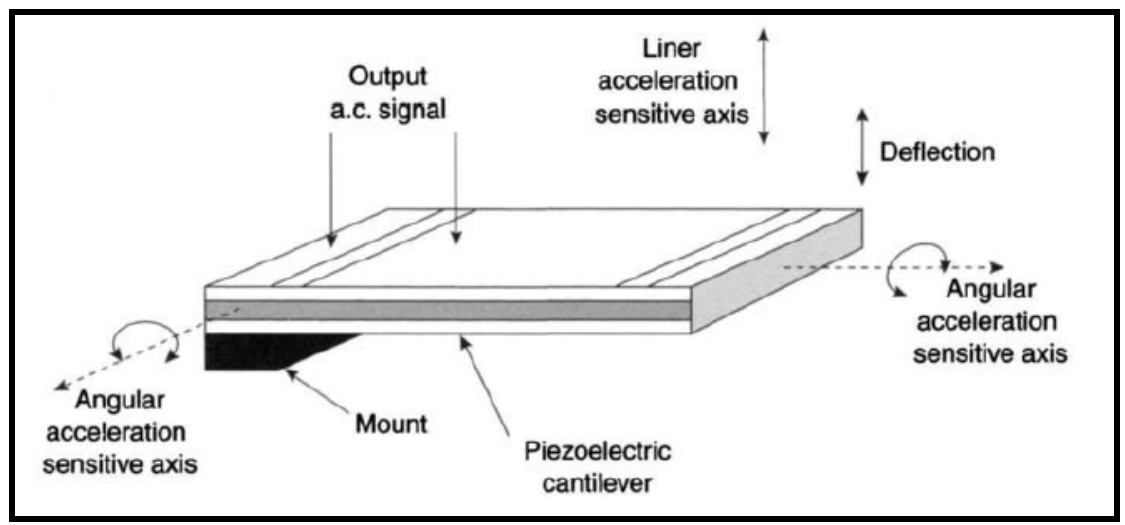

Figure 7 Piezoelectric accelerometer (Figure is taken from [11])

Tunneling accelerometers use a constant tunneling current one tunneling tip and its counterelectrode to sense displacement. When displacement occurs, a voltage is adjusted to maintain the current at a constant level. The measured voltage presents magnitude of the applied acceleration. Resolution of the tunneling accelerometers is very high. [6,11]

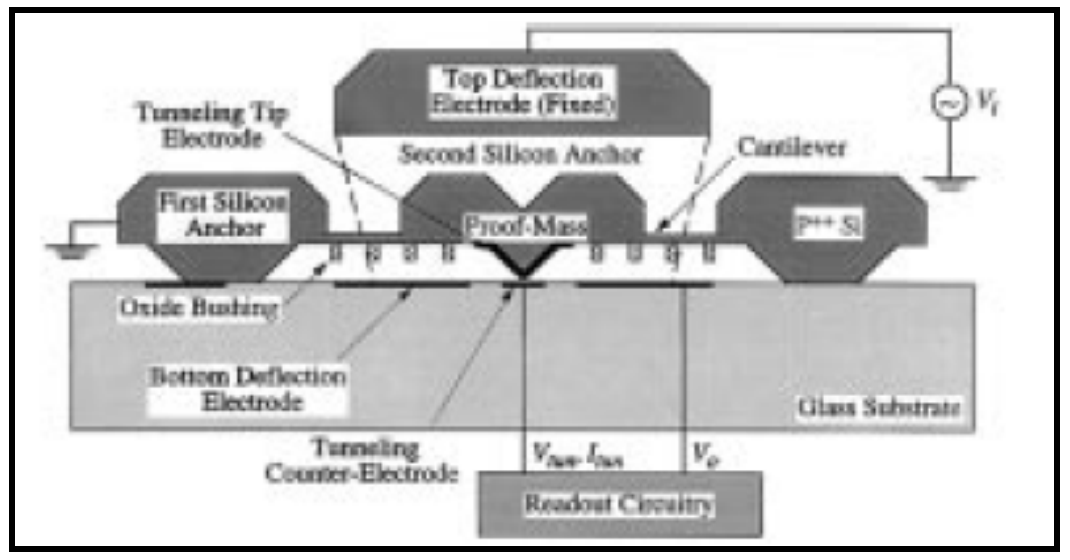

Figure 8 Tunneling accelerometer (Figure is taken from [11])

\subsubsection{Gyroscopes}

A gyroscope measures angular rates with respect to an inertial frame to fully describe the motion of a body in $3 \mathrm{D}$ space. The gyroscopes are used in a variety of roles such as stabilization, autopilot feedback, flight path sensor or platform stabilization and navigation[3]. 
Similar to accelerometers there are different types of gyroscopes. Mechanical gyros, optical gyros and MEMS gyros can be shown as an example. MEMS gyroscopes are used in this work and gyroscope error model is explained in more detail in Chapter 4.

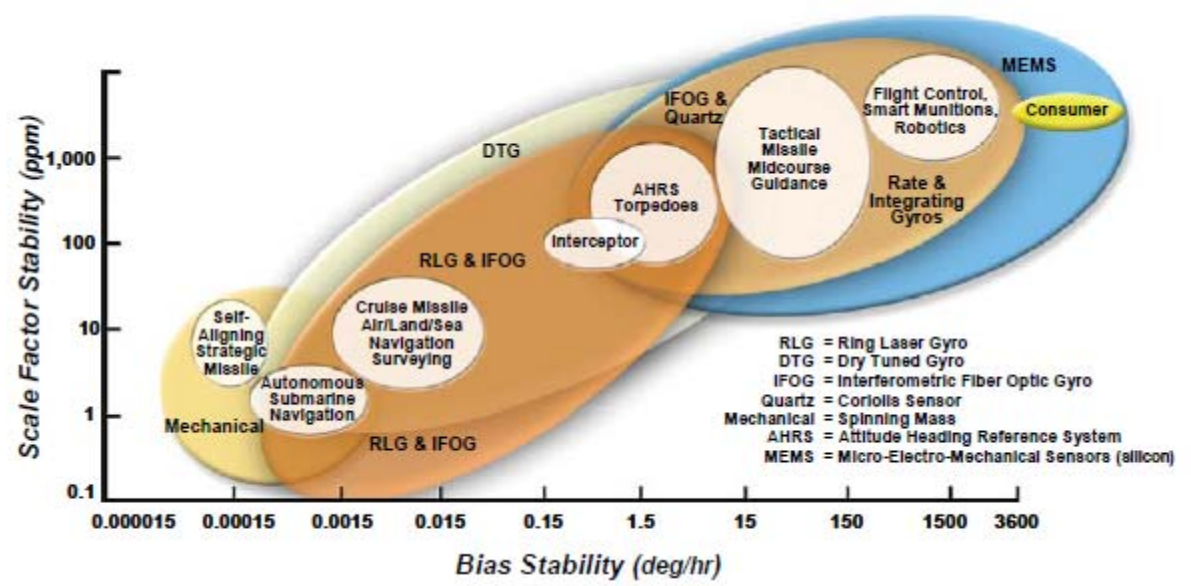

Figure 9 Current gyro technology applications (Figure is taken from [9])

MEMS sensors have potentially significant cost, size and weight advantages. Therefore, MEMS gyroscopes are widely used in tactical grade IMUs, robotics, smart munitions, etc. Scale factor stability and bias stability performance of optical gyros are significantly higher than MEMS gyros. Thus, optical gyroscopes, RLG and FOG, are used in long term navigation applications, such as cruise missiles, air/land/sea navigation surveying, etc. Some applications, strategic grade IMUs and self aligning missiles, that require high stability and low error use mechanical gyros. [9] 


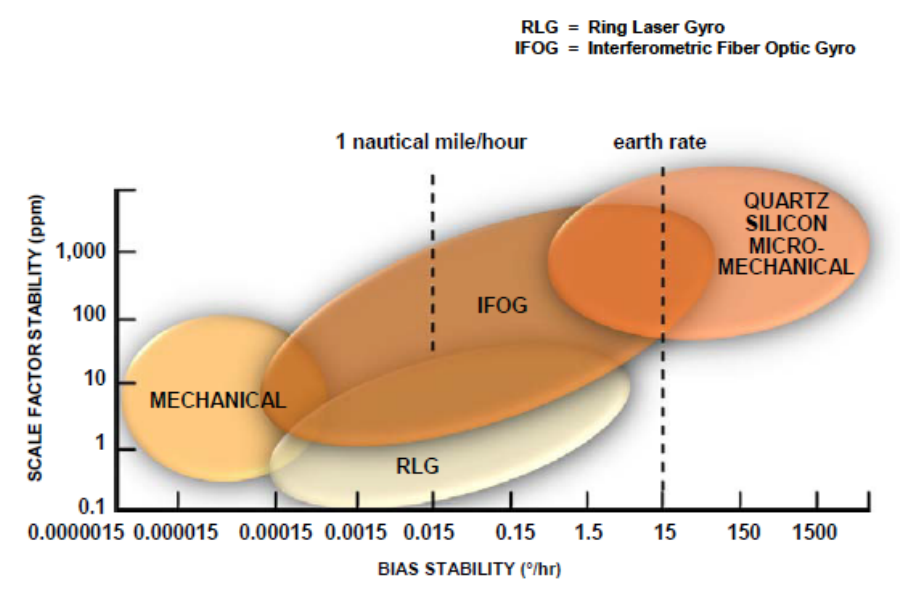

Figure 10 Near-term gyro technology applications (Figure is taken from [9])

Figure 10 shows that the MEMS and FOG gyroscope technologies are expected to replace many current systems. The performance of MEMS gyroscopes is continually improving, and they are currently being developed for many applications.

A MEMS gyroscope was used in this work. Therefore, MEMS gyroscope error model is given in more detail in Chapter 4. The following section gives detailed information about structure and working principle of MEMS gyroscopes. In addition, other types of gyroscopes are also summarized in the following section.

\subsubsection{MEMS Gyroscopes}

MEMS gyroscopes use Coriolis acceleration effect on a proof mass to detect inertial angular motion. MEMS gyroscope rely on the detection of the force acting on a mass that is subject to linear vibratory motion in a frame of reference which is rotating about an axis perpendicular to the axis of linear motion. The resulting force, acts in a direction, that is perpendicular to the both axis of vibration and the axis about which is the rotation applied [3]

MEMS gyroscopes are divided into three groups and almost all of these groups use Coriolis effect to sense angular rate. 
Structure of tuning fork gyroscope consists of two tines, which are connected to a junction bar. The tines are differentially resonated to a fixed amplitude. When the gyroscope is rotated, Coriolis force causes a differential sinusoidal force to develop on the individual tines, orthogonal to the main vibration $[6,11]$.

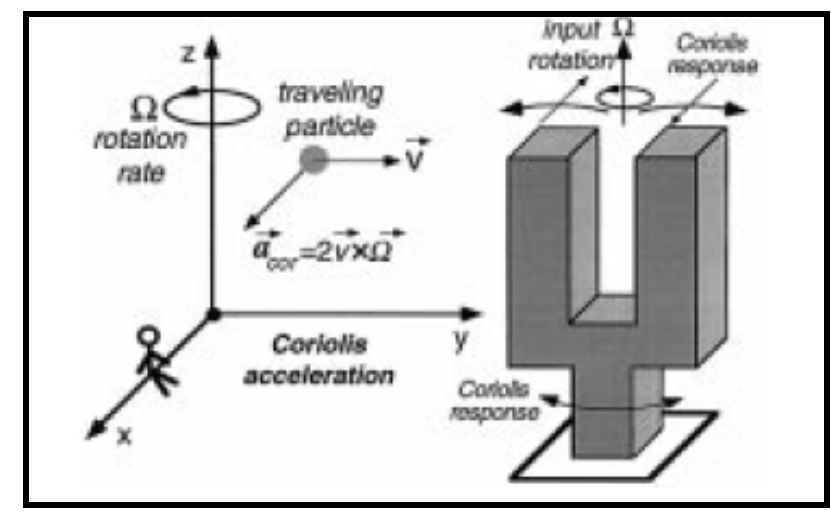

Figure 11 Tuning fork gyroscope ( Figure is taken from [11])

Vibrating wheel gyroscope includes a wheel that vibrates about of axis symmetry. Vibrating wheels operate much like the macroscopic spinning wheel gyroscope but use capacitive sensors to determine changes in attitude. The wheel rotation about the symmetry axis, results in the wheel tilting and the occurred tilt produce a change in the angular rate measurement $[6,22]$.

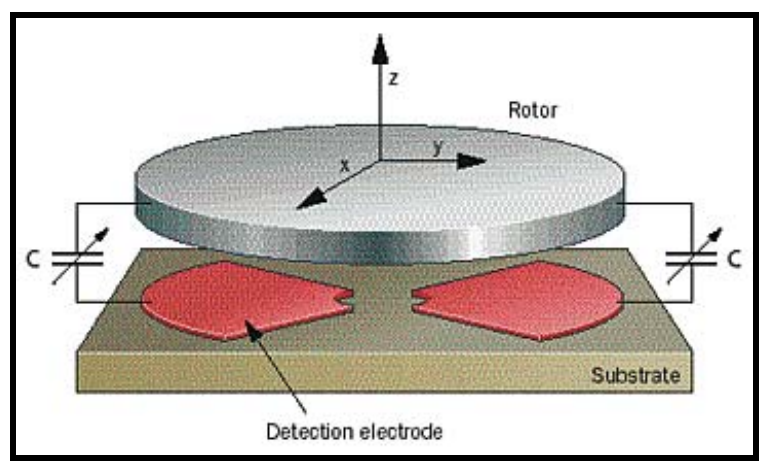

Figure 12 Vibrating wheel gyroscope (Figure is taken from [22])

In a wine glass resonator gyroscope, a wine glass resonator makes use of a hemisphere driven to resonance, the nodal points of which are measured to detect rotation $[6,22]$. Fused silica is used in the production process of wine glass resonator gyroscope. 
Other types of gyroscope technologies can be listed as mechanical gyroscopes, rate gyroscopes and optical gyroscopes (Ring laser, fiber optic) . Ring laser gyro (RLG) and fiber optic gyro (FOG) are the most popular and widely used sensors with MEMS gyroscopes. But the performance and cost of FOG and RLG are higher than the MEMS gyroscopes. According to inertial navigation system's performance requirements FOG, RLG or MEMS sensors are selected to design inertial measurement unit.
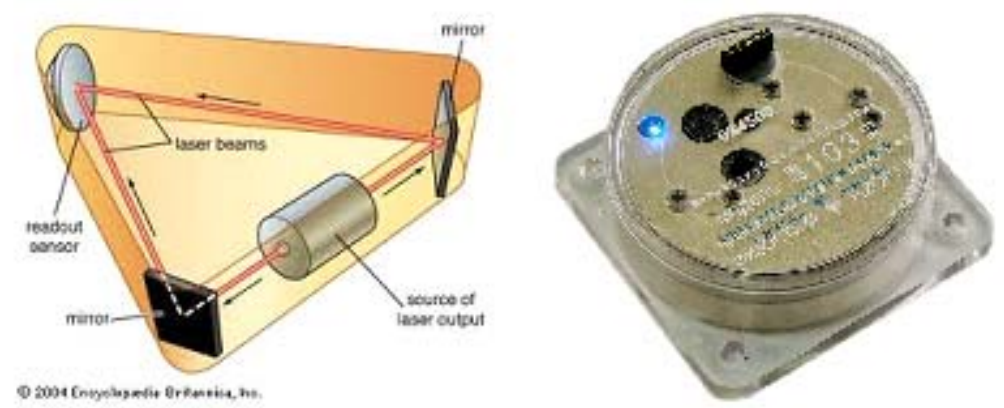

Figure 13 Ring Laser and Fiber Optic Gyroscope 


\section{CHAPTER 3}

\section{THE KALMAN FILTER}

One of the most common problems in science and engineering is the estimation of various quantities based on collecting measurements [12]. There are many ways to estimate an unknown quantity from available data such as mean square estimation, maximum-likelihood estimation, recursive estimation, wiener filtering and Kalman filter [13]. The Kalman filter is the most widely used the most advanced technique within these estimation techniques. Additionally Kalman filter has extended and improved types.

This chapter basically covers an introduction to the Kalman filter. Section 3.1 provides the basic features of the Kalman filter and Section 3.2 defines the mathematical models, filter parameters and tuning. Finally, Section 3.3 gives the role of the Kalman filter in this thesis work.

\subsection{Properties of the Kalman Filter}

The Kalman filter is a set of mathematical equations that provides an efficient computational (recursive) means to estimate the state of a process, in a way that minimizes the mean of the squared error [14]. In general, the Kalman filter models the state dynamics as a linear function of the previous state, the control input and system dynamics zero-mean Gaussian noise. In addition to that the Kalman filter can be explained as a data processing algorithm by using limited number of variables. Only the new measurement data need to be processes on each iteration [15]. Therefore the memory requirement was reduced with the use of KF algorithm and 
this is the one of the most important feature of the KF. Besides, the Kalman filter is preferred for real-time applications such as navigation systems, radar systems, GPS, etc....

- The KF includes five major elements. These are system model, measurement model, state vector, measurement vector and error covariances [15].

- The state vector is a group of parameters which are estimated by Kalman filter and the state vector cannot be measured directly.

- The system model describes how the KF states and error covariance matrix vary with time.

- The measurement model specifies the mathematical relationship between the system state and the measurements.

- The measurement vector contains instantaneous measurement values according to the measurement model.

- Error covariances represent the uncertainties in the state estimates and the measurements.

\subsubsection{System Model}

The system model equation describes a system with noise and this equation called as discrete stochastic dynamical equation [13].

$$
x_{k}=A x_{k-1}+B u_{k-1}+w_{k-1}
$$

The state transition matrix , $A$, defines how the state vector changes with time. Similarly, the transition matrix for control input, $B$, determines transition between the system and control input. The random variable $w_{k}$ represent the process noise and it is assumed to be white and with normal probability distribution [13, 14]. Detailed information about system model equation parameters are given in Table-2. 
Table 2 System Model Parameters

\begin{tabular}{|c|c|}
\hline Parameters & Definitions \\
\hline$A$ & State transition matrix \\
\hline$B$ & Transition matrix for control input \\
\hline$x_{k}$ & State vector \\
\hline$u_{k-1}$ & Control input \\
\hline$w_{k-1}$ & Process noise component \\
\hline
\end{tabular}

$$
p(w) \sim N(0, Q)
$$

Q: Process noise covariance

\subsubsection{Measurement Model}

The measurement model equation which is given in equation (3.3) describes the relation between states and observable system output.

$$
z_{k}=C x_{k}+v_{k}
$$

The conversion matrix , $C$, maps the state into the measurement. The measurement noise, $v_{k}$, is caused by instrumentation errors and it is assumed to be white and with normal probability distribution. $[12,13]$

$$
\begin{gathered}
p(v) \sim N(0, R) \\
R: \text { Measurement noise covariance }
\end{gathered}
$$

Detailed information about system model equation parameters are given in Table-3. 
Table 3 Measurement Model Parameters

\begin{tabular}{|c|c|}
\hline Parameters & Definitions \\
\hline$C$ & $\begin{array}{c}\text { Conversion matrix between the system } \\
\text { and measurement }\end{array}$ \\
\hline$z_{k}$ & Measurement \\
\hline$v_{k}$ & Measurement noise \\
\hline
\end{tabular}

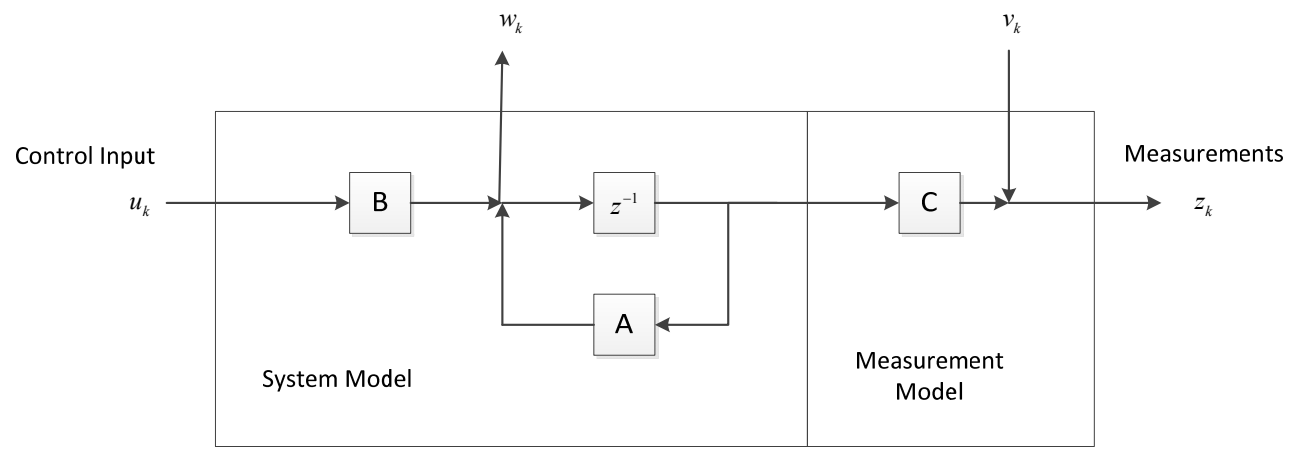

Figure 14 System and Measurement Model Implementation [13]

\subsection{The Mathematical Model of The Kalman Filter}

Basically, the equations for the Kalman filter divided into two groups. These groups are time update equations and measurement update equations. The Kalman filter uses time update and measurement update equations recursively to estimate the system state.

\subsubsection{Time Update Equations}

Prediction of the next state of the system is calculated by processing the time update equations. Moreover, error covariance which defines the probable error in the algorithm's estimate of the state vector is also calculated by using time update equations[16]. In summary, time update equations compute a priori quantities for the next time step in absence of measurements. 


$$
\begin{aligned}
& \hat{x}_{k}^{-}=A \hat{x}_{k-1}+B u_{k-1} \\
& P_{k}^{-}=A P_{k-1} A^{T}+Q
\end{aligned}
$$

Table 4 Time Update Equations Parameter Definitions

\begin{tabular}{|c|c|}
\hline Parameters & Definitions \\
\hline$\hat{x}_{k}^{-}$ & A priori state estimate \\
\hline$P_{k}^{-}$ & A priori error covariance \\
\hline
\end{tabular}

Equation (3.5) and (3.6) constitute time update equations. Outputs of these equations are used as input for the measurement update equations.

\subsubsection{Measurement Update Equations}

The measurement equations provide a correction based on measurements. The purpose of these equations is to correct a priori estimates with measurements and compute a posteriori state estimate.

$$
\begin{gathered}
K_{k}=P_{k}^{-} C^{T}\left(C P_{k}^{-} C^{T}+R\right)^{-1} \\
\hat{x}_{k}=\hat{x}_{k}^{-}+K_{k}\left(z_{k}-C \hat{x}_{k}^{-}\right) \\
P_{k}=\left(I-K_{k} C\right) P_{k}^{-}
\end{gathered}
$$

Detailed information about parameters of measurement update equations are given in Table-5. 
Table 5 Measurement Update Equations Parameter Definitions

\begin{tabular}{|c|c|}
\hline Parameters & Definitions \\
\hline$K_{k}$ & Kalman gain \\
\hline$\hat{x}_{k}$ & A posteriori state estimate \\
\hline$P_{k}$ & A posteriori error covariance \\
\hline
\end{tabular}

Equation (3.7) represents the Kalman gain. The function of the Kalman gain is to minimize the error caused by the difference between prediction and measurement. In other words, Kalman gain is the weighting which determines the influence of the residual in updating the estimate [13]. Equation (3.8) gives the updated version of the state estimation. The output of the equation (3.5) is corrected by acquired measurements and corrected a posteriori state estimation is calculated. Similarly, equation (3.9) shows the correction method of error covariance matrix $P_{k}$.

\subsubsection{Kalman Filter Algorithm}

The Kalman filter algorithm consists of merely predicting the errors in the state, and then once new observations have been taken, correcting these state errors to obtain an optimal solution. Basically, the Kalman filter algorithm depends on predictioncorrection loop and initial conditions. The time update equations need previous time step's state estimation and error covariance to produce a priori estimates for the next

time step. Therefore initial conditions for state and error covariance $\left(\hat{x}_{0}, P_{0}\right)$ must be determined before starting the algorithm.

The diagonal elements of error covariance matrix are the variances of the each state estimate error, while their roots are uncertainties in the estimate of the state vector. The off diagonal elements are the covariances, represents the correlations between the errors $[12,13,16]$. 
If elements of the $P$ matrix are large, the Kalman gain will be large. It means that the state uncertainties must be reduced by weighting the state estimates toward the new measurement data.

The Kalman filter estimates will change quickly as they converge with the true values of the states, so the state uncertainty will drop rapidly. However, measurement noise covariance causes the the Kalman gain drop, weighting the state estimates more toward their previous sates. This reduces the rate at which states change, so the reduction in the state uncertainty slows. Eventually, the Kalman filter will approach equilibrium. At equilibrium, the state estimates may still vary, but the level of confidence in those estimates, reflected by the state uncertainty, will be more or less fixed $[14,15,16]$. The equilibrium behavior of the Kalman gain and the error covariance matrix can be observed from equation (3.10) and (3.11). Additionally Figure 15 represents the typical algorithm behavior of the Kalman gain and Figure 16 shows the typical algorithm behavior of the the error covariance matrix. Finally, Figure 17 summarizes the recursive structure of the Kalman filter.

$$
\begin{gathered}
K_{k}=\frac{P_{k}^{-} C^{T}}{C P_{k}^{-} C^{T}+R} \\
\lim _{P_{k}^{-} \rightarrow 0} K_{k}=0
\end{gathered}
$$

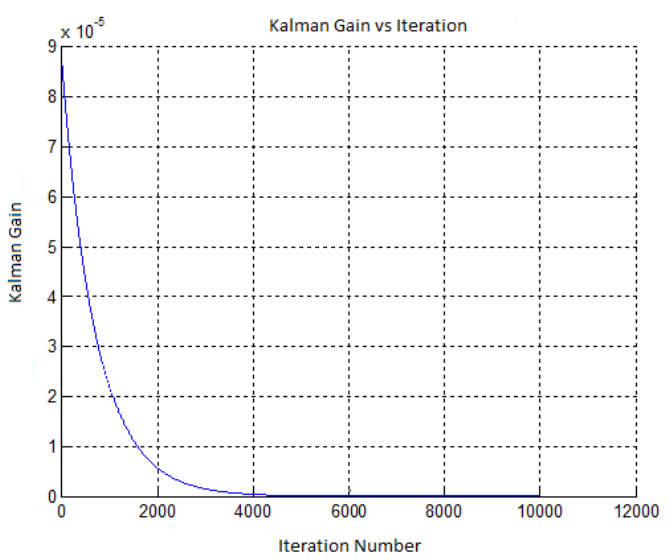

Figure 15 Kalman Gain vs Iteration 


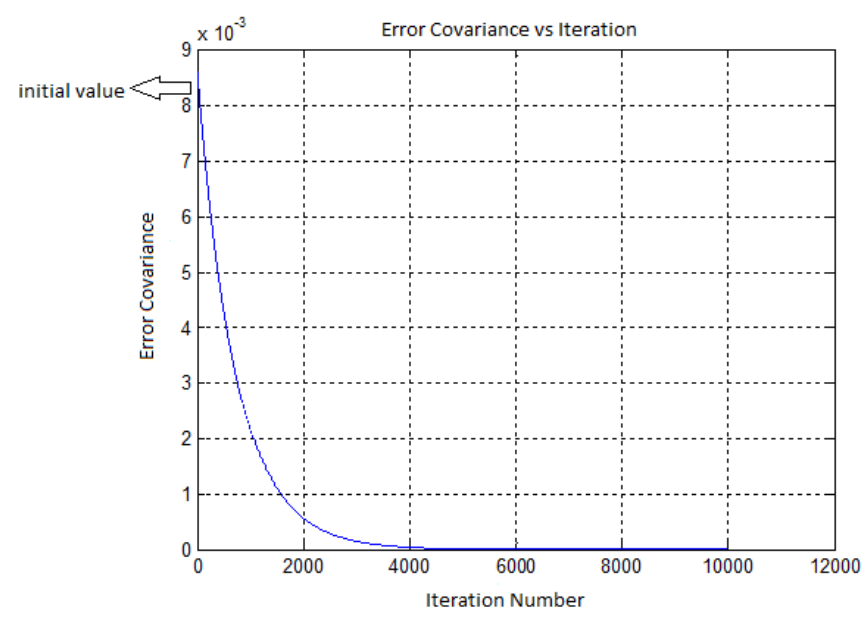

Figure 16 Error Covariance vs Iteration

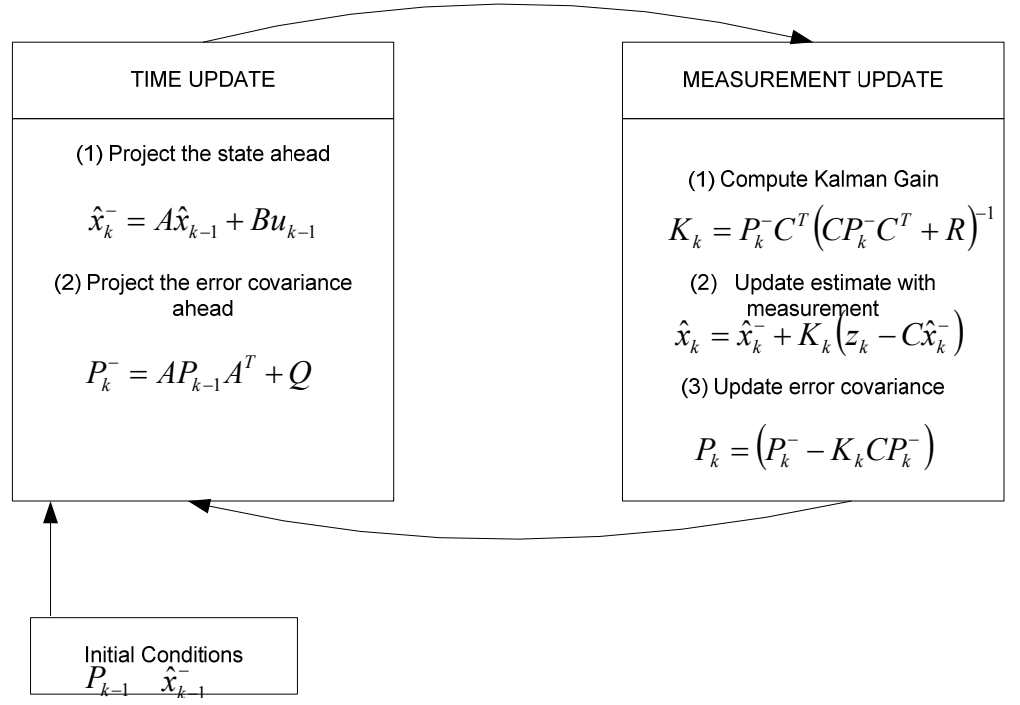

Figure 17 Recursive structure of the Kalman filter [14]

\subsection{The Role of the Kalman Filter In This Thesis Study}

As mentioned in the previous chapters, inertial measurement units have two types of error. Deterministic errors can be determined by performing the calibration test procedure and processing the test data. Furthermore, stochastic errors of the sensors should be estimated by using some techniques. 
The Kalman filter method is commonly used in real time applications and the Kalman filter estimation technique is the most appropriate technique to estimate gyroscope and accelerometer bias instability due to its properties such as low memory requirement, optimal estimation and high performance in noisy measurements. (Assuming the system is linear.)

Gyroscope and accelerometer time constant and data sampling interval were defined constant in this thesis study. Additionally, gyroscope and accelerometer bias instabilities were modeled with discrete Gauss-Markov Model. The Gauss Markov model becomes linear when the time constant and sampling interval are constant. Therefore the Kalman filter algorithm was used as estimation algorithm in this thesis study. Furthermore, this thesis study will be adapted to practical applications. Thus, computational complexity becomes very important design parameter. In addition to other advantages of the Kalman filter, computational complexity of the algorithm is not high. For this reason, Kalman filter algorithm is suitable for practical and real applications. 


\section{SENSOR ERROR PARAMETERS AND IMU ERROR MODELS}

This chapter explains MEMS accelerometer and MEMS gyroscope error parameters. Section 4.1 covers accelerometer error parameters and MEMS accelerometer error model. Section 4.2 discusses gyroscope error parameters and MEMS gyroscope error model. Additionally, IMU error model is also given in this chapter.

\subsection{Inertial Sensor Error Types}

The inertial sensor errors can be classified into two groups, deterministic (systematic) and stochastic (random) errors [17]. The deterministic errors are defined by static and dynamic calibration tests. Besides, stochastic errors can be estimated by using several estimation techniques. Estimation methodology of the deterministic errors, calibration tests and least squares fitting method, are discussed in more detail in Chapter 5. Modeling and estimation of the stochastic errors are also explained in Chapter 6.

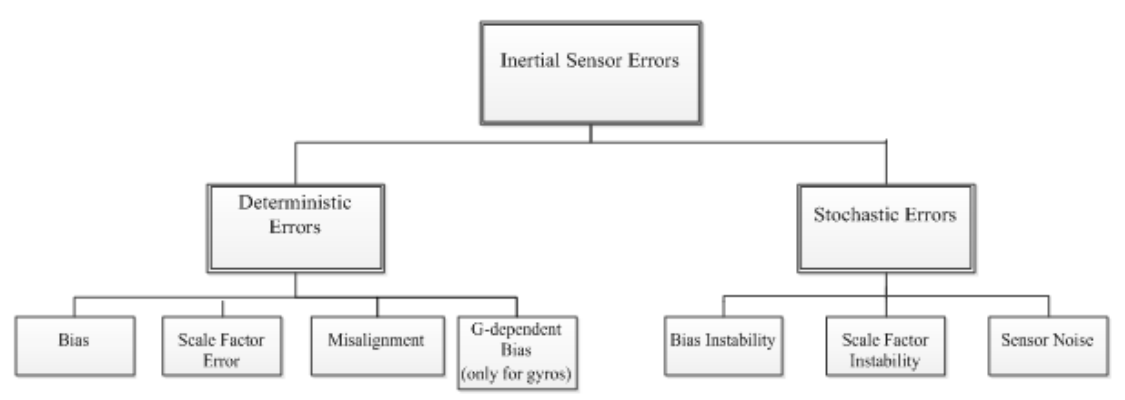

Figure 18 Major types of inertial sensors (Figure is taken from[6]) 


\subsubsection{Deterministic Error Parameters of Accelerometers}

Accelerometers have three dominant deterministic errors which are scale factor error, bias and misalignment.

\subsubsection{Bias}

Bias (offset) is the accelerometer output at zero $g$ [18]. It means that when no input acceleration is applied to the sensor, measured acceleration presents bias. Unit of accelerometer bias is mili-g. The bias includes fixed terms, temperature induced variations, turn-on to turn-on variations and in-run variations [19]. Fixed terms of bias and temperature induced variations in bias can be estimated by laboratory calibration tests and estimated fixed bias and temperature induced variations are used as an input of error compensation algorithms. If the accelerometer bias is not compensated, error in velocity and position grow with time.

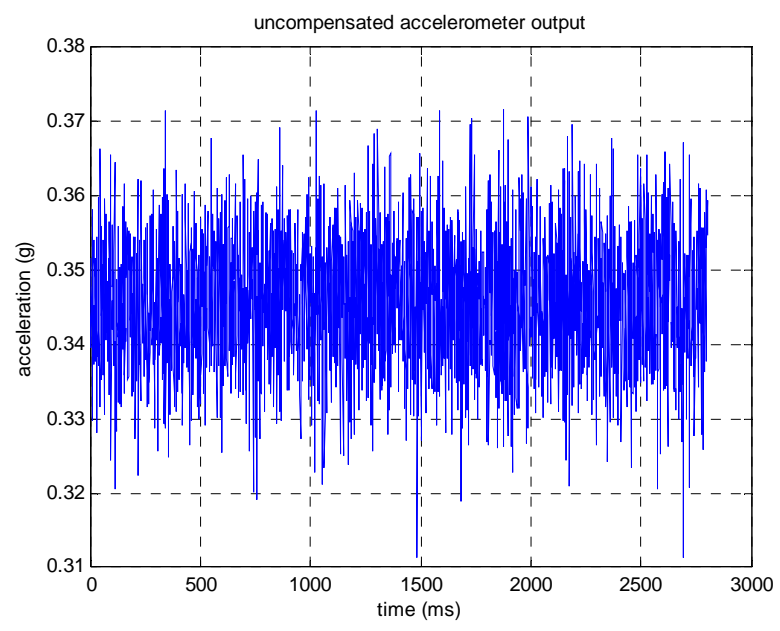

Figure 19 Accelerometer output with bias at zero $g$ 


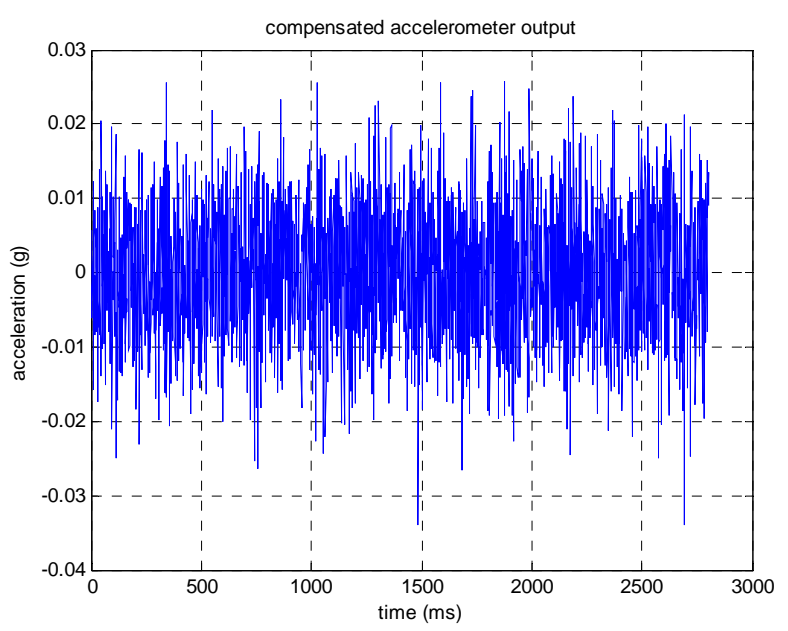

Figure 20 Accelerometer output without bias at zero $g$

\subsubsection{Scale Factor Error}

Scale factor error is errors in the ratio of a change in the output signal to a change in the input acceleration which is to be measured [3]. The magnitude of the scale factor is expressed in parts per million (ppm) or percent. The scale factor error includes fixed terms, temperature induced variations, asymmetry and nonlinearity error parts. The major parts of scale factor error are fixed term and temperature induced variations. Similar to the bias, scale factor errors can be estimated by laboratory calibration tests and estimated error parameters constitute input of error compensation or calibration algorithms.

\subsubsection{Misalignment}

Orthogonality error. Accelerometers should be mounted orthogonal to observe true measurements about its sensitivity axis. But mechanical components cannot be produced perfectly and these components cannot be mounted perfectly. This will cause a nonorthogonality between the IMU axes and this nonorthogonality creates a scale factor effect on measurements. Any movements in any axis causes a change in the other axes depending on the magnitude of misalignment. The unit of 
misalignment is mili-radian and the misalignment error can be determined by laboratory calibration tests and used in error compensation algorithms.

\subsubsection{Deterministic Error Parameters of Gyroscopes}

Gyroscopes have four dominant deterministic errors which are scale factor error, bias, misalignment and g-dependent bias (acceleration dependent bias).

\subsubsection{Bias}

The gyroscope bias can be defined as the gyroscope output in the absence of an applied angular rate. It means that when no input angular rate is applied to the sensor, measured angular rate presents bias. Unit of gyroscope bias is deg/h. Gyro bias and accelerometer bias have similar properties. For example, contents of gyroscope and accelerometer bias are the same. The gyro bias includes fixed terms, temperature induced variations, turn-on to turn-on variations and in-run variations like accelerometer bias. In-run bias variations represent stochastic error part of the bias. Fixed terms of bias and temperature induced variations in bias can be estimated by dynamic laboratory calibration tests and estimated fixed bias and temperature induced variations are used as an input of error compensation algorithms.

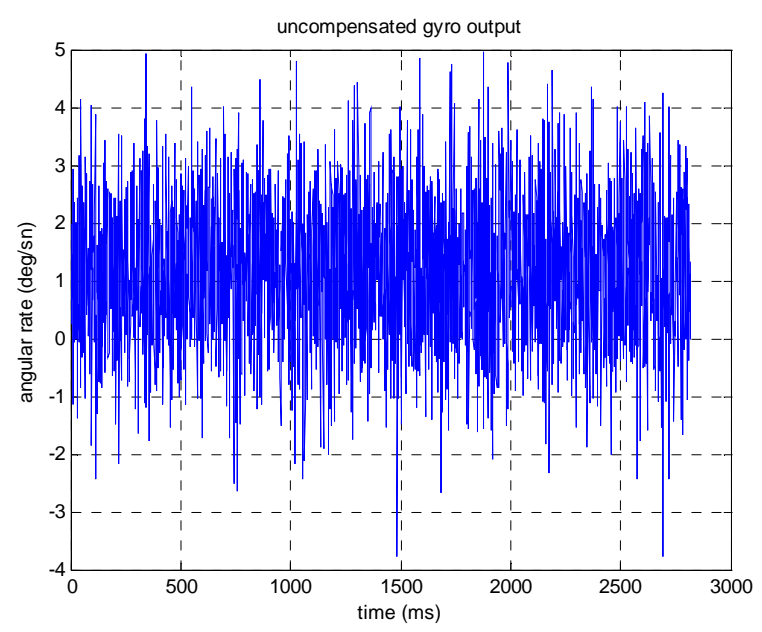

Figure 21 Gyro output with bias at zero rotational input 


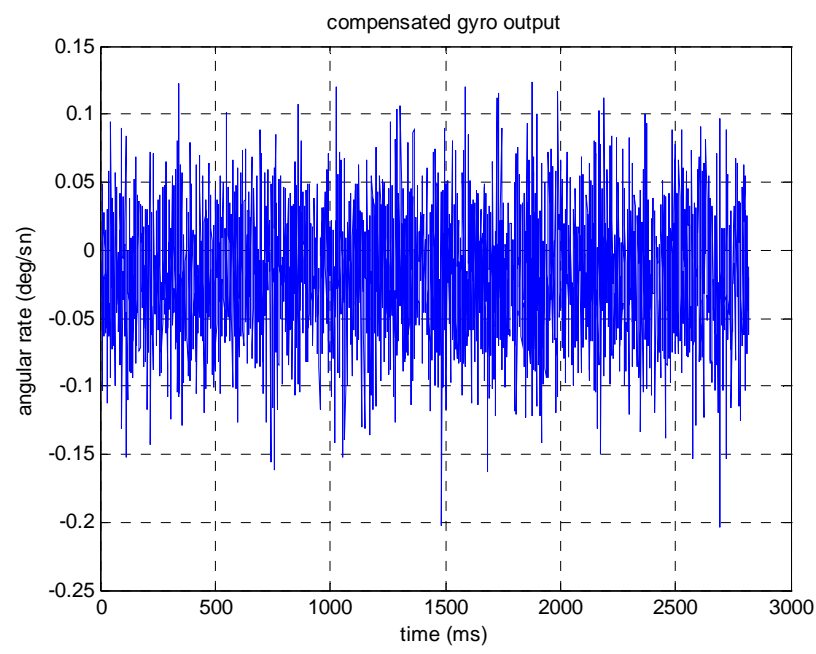

Figure 22 Gyro output without bias at zero rotational input

\subsubsection{Scale Factor Error}

Scale factor is the ratio between input and output. The scale factor value for the perfect sensor is 1. But the scale factor which is calculated from real sensor measurements has a difference between the ideal value 1 and this difference represents scale factor error. The magnitude of the scale factor is expressed in parts per million (ppm). The gyroscope scale factor error includes fixed terms, temperature induced variations, asymmetry and nonlinearity error parts. The major parts of the gyroscope scale factor error are fixed term and temperature induced variations. Similar to the bias, scale factor errors can be estimated by laboratory calibration tests and estimated error parameters constitute input of error compensation or calibration algorithms. In addition to that effect of the scale factor error becomes observed at high rates.

It can be observed from Figure 21 total gyroscope error is approximately $1.5 \mathrm{deg} / \mathrm{sn}$. According to Figure 23 total gyroscope error is $3 \mathrm{deg} / \mathrm{sn}$. The difference between Figure 21 and Figure 23 comes from the observability of scale factor error. 


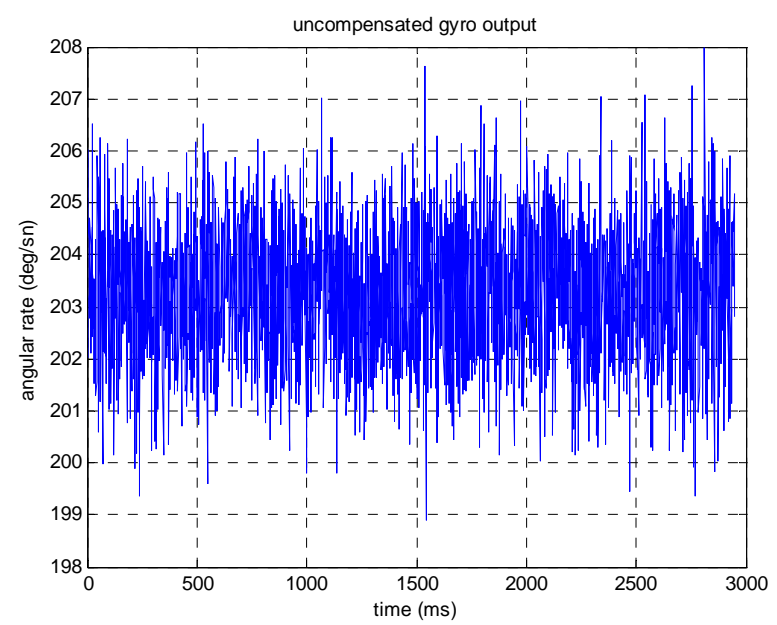

Figure 23 Gyro output with bias and scale factor error at $200 \mathrm{deg} / \mathrm{sn}$

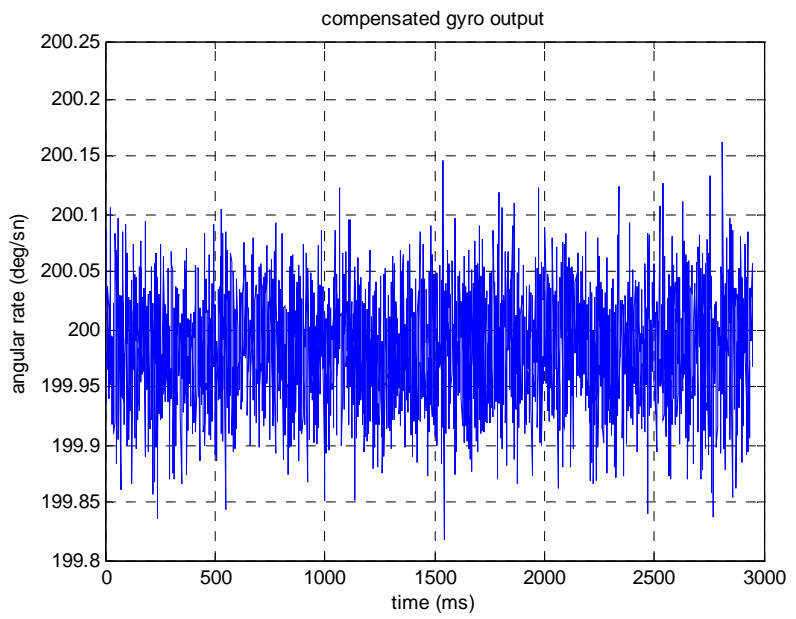

Figure 24 Gyro output without bias and scale factor error at $200 \mathrm{deg} / \mathrm{sn}$

\subsubsection{Misalignment}

The explanation given for the accelerometer is valid for gyros.

\subsubsection{G-dependent bias}

Acceleration sensitive bias. Structure of the MEMS gyroscope is affected by acceleration. 
Therefore this effect causes an offset in the output signal. This bias component is proportional to the acceleration magnitude which is applied about the measurement axis [3]. Therefore there is a relationship between acceleration and gyroscope measurement and this magnitude of this relationship is determined with g-dependent bias coefficient. Like other deterministic errors, g-dependent bias coefficients can be determined by laboratory tests and these coefficients are used in calibration algorithms. Unit of the g-dependent bias is $\mathrm{deg} / \mathrm{h} / \mathrm{g}$.
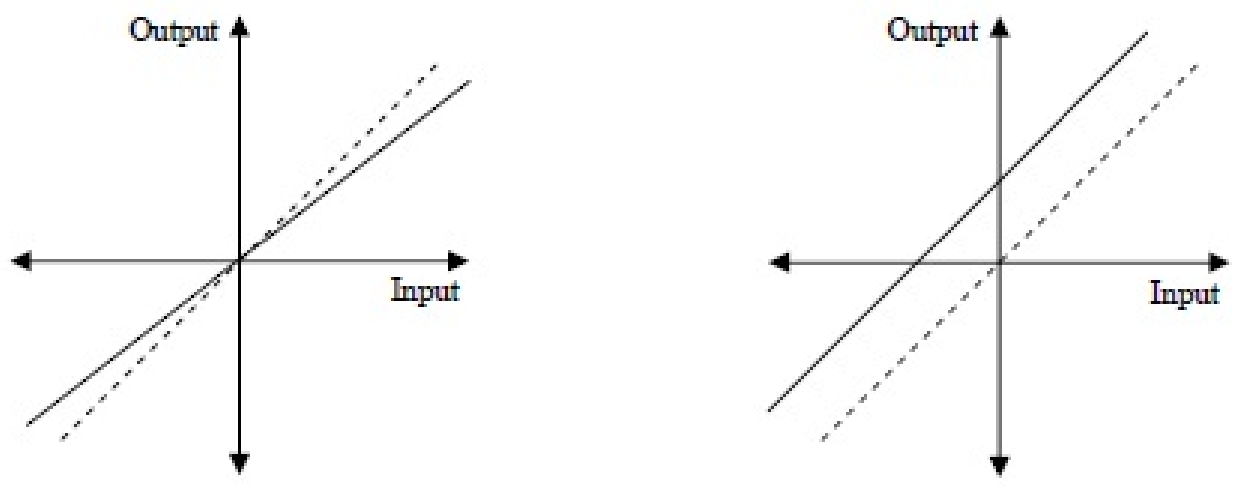

Figure 25 Representation of Scale Factor and Bias (Figure is taken from[20])

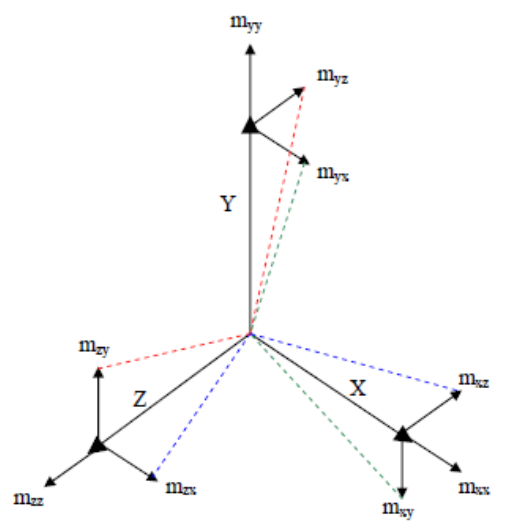

Figure 26 Representation of Misalignment (Figure is taken from[20]) 


\subsubsection{Stochastic Error Parameters of Accelerometers and Gyroscopes}

Stochastic errors are the random errors that occur due to random variations of bias or scale factor drift over time and random sensor noise [6]. Random variations in bias and scale factor are the low frequency components of the stochastic errors. The sensor noise is also high frequency components of the stochastic errors. The most important feature of stochastic errors is there may not be any direct relationship between input and output [21]. The source of the stochastic errors are flicker noise in the electronics and interference effects on signals. Allan variance tests and autocorrelation analysis are performed to determine the stochastic characterization of inertial sensors. In addition to that several random processes exist for modelling stochastic errors.

\subsubsection{Bias Instability (Bias Drift)}

This error occurs due to change in bias during a run [3]. In other words, bias instability represents the variations in bias which change with time. Bias instability can be characterized by Allan variance and autocorrelation analysis and modeled by using results of these tests and analysis. Various methods (random processes) such as Random Walk Model, Gauss-Markov Model, Random Constant Model and Autoregressive Model are used to model stochastic errors. First order Gauss-Markov Model is the most selected and the most appropriate random process for modeling bias instability [20,21]. Therefore the random process Gauss- Markov Model was used to model gyro and accelerometer bias instability in this thesis work. Stochastic model of the bias instability is presented in equation (4.1), (4.2) and (4.3). The bias instability of sensors is denoted by $1 \sigma$ value. It means that this error type has Gaussian distribution.

$$
\begin{gathered}
x_{k}=e^{-d t / T_{c}} x_{k-1}+w_{k} \\
d t: \text { sampling period } \\
T_{c}: \text { sensor time constant } \\
w_{k}: \text { driven noise }
\end{gathered}
$$




$$
\begin{gathered}
\sigma_{x_{k}}^{2}=\frac{\sigma_{w_{k}}^{2}}{1-e^{-2 d t / T_{c}}}, \text { bias instability variance } \\
\sigma_{w_{k}}^{2}=\sigma_{x_{k}}^{2}\left(1-e^{-2 d t / T_{c}}\right), \text {, driven noise variance }
\end{gathered}
$$

\subsubsection{Scale Factor Instability (Scale Factor Drift)}

Scale factor instability represents the variations in scale factor which change with time. Scale factor instability characterization test is different from the bias instability characterization test method. Scale factor instability characterization needs long term dynamical rate test and the effect of the scale factor instability is not very observable and quite negligible. Therefore, this error component excluded from the scope of this thesis study.

\subsubsection{Random Sensor Noise}

High frequency component of the stochastic errors. Effect of sensor noise can be reduced by filtering. Low pass filters are designed according to sensor noise bandwidth and noise power. Random sensor noise is modeled as a zero mean white noise in the IMU error models. And these system specific filters are used in error compensation algorithms. Unit of the random sensor noise density is $\mathrm{deg} / \mathrm{h} / \sqrt{\mathrm{Hz}}$ or $\mathrm{deg} / \mathrm{s} / \sqrt{\mathrm{Hz}}$ and the random noise density is represented by $1 \sigma$ value.

\subsubsection{Other Types of Errors}

Temperature dependent bias and scale factor variations, scale factor asymmetry, scale factor linearity error and sensor based misalignment errors are the other types of inertial sensor types of errors. Temperature dependent variations can be determined by temperature calibration tests and drift in bias and scale factor, caused by temperature changes, are modeled with second or higher order equations. These 
equations are added to error compensation algorithms to correct temperature dependent variations. Scale factor asymmetry represents the difference between the scale factor measured with positive input and negative input. And scale factor linearity error represents difference between the scale factor measured with high rate or acceleration and low rate or acceleration.

It was assumed that, inertial sensor errors, bias and scale factor, did not include temperature dependent variations, scale factor asymmetry and scale factor linearity.

\subsection{IMU Error Model}

Inertial navigation systems need acceleration and angular rate measurements in the $\mathrm{x}$, $\mathrm{y}$ and $\mathrm{z}$ - directions to calculate attitude, position and velocity. Therefore, inertial measurement units contain three accelerometer and three gyroscope. For this reason, IMU error model is determined with equation (4.5) and (4.7).

\subsubsection{Accelerometer Error Model}

The error model of the single axis accelerometer is given by:

$$
\tilde{a}_{x}=\left(1+S_{x}+\delta S_{x}\right) a_{x}+B_{x}+\delta B_{x}+n_{x}
$$

$$
\begin{aligned}
& \tilde{a}_{x}: \text { accelerometer output } a_{x}: \text { actual acceleration } \\
& S_{x}: \text { scale factor error } \\
& B_{x}: \text { bias } \\
& \delta S_{x}: \text { scale factor instability } \\
& \delta B_{x}: \text { bias instability } \\
& n_{x}: \text { sensor noise }
\end{aligned}
$$

The error model of three accelerometer which are mounted on $\mathrm{x}, \mathrm{y}$ and $\mathrm{z}$ axis of an IMU is represented by equation (4.5). 


$$
\left[\begin{array}{c}
\tilde{a_{x}} \\
\tilde{a_{y}} \\
\tilde{a_{z}}
\end{array}\right]=\left[\begin{array}{ccc}
1+S_{x}+\delta S_{x} & M_{x y} & M_{x z} \\
M_{y x} & 1+S_{y}+\delta S_{y} & M_{y z} \\
M_{z x} & M_{z y} & 1+S_{y}+\delta S_{y}
\end{array}\right]\left[\begin{array}{l}
a_{x} \\
a_{y} \\
a_{z}
\end{array}\right]+\left[\begin{array}{l}
B_{x}+\delta B_{x} \\
B_{y}+\delta B_{y} \\
B_{z}+\delta B_{z}
\end{array}\right]+\left[\begin{array}{l}
n_{x} \\
n_{y} \\
n_{z}
\end{array}\right]
$$

$$
M_{x y}, M_{x z}, M_{y x}, M_{y z}, M_{z x}, M_{z y}: \text { Misalignment Errors }
$$

\subsubsection{Gyroscope Error Model}

The error model of the single axis gyroscope is given by:

$$
\tilde{w}_{x}=\left(1+S_{x}+\delta S_{x}\right) w_{x}+B_{x}+\delta B_{x}+B_{G x} a_{x}+n_{x}
$$

$$
\begin{aligned}
& \tilde{w}_{x}: \text { gyroscope output } w_{x}: \text { actual angular rate } \\
& S_{x}: \text { scale factor error } \\
& B_{x}: \text { bias } \\
& \delta S_{x}: \text { scale factor instability } \\
& \delta B_{x}: \text { bias instability } \\
& B_{G x}: \text { g-dep bias coeff. } \\
& n_{x}: \text { sensor noise }
\end{aligned}
$$

The error model of three gyroscope which are mounted on $\mathrm{x}, \mathrm{y}$ and $\mathrm{z}$ axis of an IMU is represented by:

$$
\begin{gathered}
{\left[\begin{array}{c}
\tilde{w_{x}} \\
\tilde{w_{y}} \\
\tilde{w_{z}}
\end{array}\right]=\left[\begin{array}{ccc}
1+S_{x}+\delta S_{x} & M_{x y} & M_{x z} \\
M_{y x} & 1+S_{y}+\delta S_{y} & M_{y z} \\
M_{z x} & M_{z y} & 1+S_{y}+\delta S_{y}
\end{array}\right]\left[\begin{array}{l}
w_{x} \\
w_{y} \\
w_{z}
\end{array}\right]+\left[\begin{array}{c}
B_{x}+\delta B_{x} \\
B_{y}+\delta B_{y} \\
B_{z}+\delta B_{z}
\end{array}\right]+\left[\begin{array}{ccc}
B_{g x} & 0 & 0 \\
0 & B_{g y} & 0 \\
0 & 0 & B_{g z}
\end{array}\right]\left[\begin{array}{l}
a_{x} \\
a_{y} \\
a_{z}
\end{array}\right]+\left[\begin{array}{l}
n_{x} \\
n_{y} \\
n_{z}
\end{array}\right]} \\
M_{x y}, M_{x z}, M_{y x}, M_{y z}, M_{z x}, M_{z y}: \text { Misalignment Errors }
\end{gathered}
$$


Equation (4.5) and (4.7) give the error characterization of inertial measurement units. An IMU Error Model was developed and implemented within the scope of this thesis work. MATLAB Simulink software was used to construct equation (4.5) and (4.7). Effects of inertial sensor errors were simulated by using constructed equations. Simulation outputs were processed by least squares estimation algorithm, which is explained in Chapter 5, to estimate IMU deterministic error parameters. Additionally, IMU Error Model's simulation outputs were used as input for Deterministic Error Compensation algorithm/model. 


\section{CHAPTER 5}

\section{ESTIMATION OF DETERMINISTIC ERROR PARAMETERS}

This chapter suggests some test methods about estimation of deterministic error parameters. Sections 5.1.1 and 5.1.2 summarize basic laboratory calibration test methods and data processing procedure. Sections 5.1.3, 5.1.4 and 5.1.5 explain the improved calibration test procedure which is implemented in the scope of this thesis work. Compensation algorithms aim of compensation algorithms. Finally, deterministic error compensation algorithm is provided in Section 5.2.

\subsection{Laboratory Calibration Tests}

Calibration is the process of comparing inertial sensor outputs with known input motion and determining the deterministic error parameters $[3,6]$. The output signals from the IMU are recorded during the test period. Then the collected data are processed by various methods and deterministic error parameters are determined. The six position static method and rate tests are usually described in the literature. (static acceleration test, static rate tests, multi-position tests). In addition to these test methods multi-position static and multi-rate tests are developed and explained in this thesis work. 


\subsubsection{Six Position Static Tests}

The local gravity and the Earth's rotational rate are the reference inputs for this test method. The six position static test data is used to extract accelerometer bias and scale factor. Also, according to the sensor quality, six position static test can be sufficient to estimate gyroscope bias and scale factor. For example, tactical grade IMU's gyroscopes can sense Earth's rotational rate, for this reason six position static test can be used to estimate gyroscope scale factor and bias. However automotivegrade gyroscope's bias instability and noise levels mask the reference signal, Earth's rotational rate [6]. Therefore this calibration method is not suitable for automotivegrade and some tactical grade MEMS gyroscopes[21].

The procedure of six position static method is given in the following steps :

First step: The IMU is mounted on a leveled table with each axis pointing alternately up and down position.

Second step: The gyroscope and accelerometer data is collected and recorded when the $\mathrm{x}$-axis is pointed upward. $\left(\tilde{w_{x}^{u p}}, \tilde{a_{x}^{u p}}\right)$

Third step: The gyroscope and accelerometer data is collected and recorded when the $\mathrm{x}$-axis is pointed downward. $\left(w_{x}^{\tilde{\text { down }}}, \tilde{a}_{x}^{\tilde{\text { down }}}\right)$

Fourth step: Repeat the second step for y- axis. $\left(\tilde{w_{y}^{u p}}, \tilde{a_{y}^{u p}}\right)$

Fifth step: Repeat the third step for y-axis. $\left(w_{y}^{\tilde{\text { down }}}, a_{y}^{\tilde{d o w n}}\right)$

Sixth step: Repeat the second step for $\mathrm{z}-$ axis. $\left(\tilde{w_{z}^{u p}}, \tilde{a_{z}^{u p}}\right)$

Seventh step: Repeat the third step for z-axis. $\left(w_{z}^{\tilde{d o w n}}, a_{z}^{\tilde{d o w n}}\right)$ 


\subsubsection{Data Processing Methods for Six Position Test}

Gyroscope errors can be determined by the following equations in basic processing:

$$
\begin{gathered}
B_{x}=\frac{A v\left(\tilde{w_{x}^{u p}}\right)+A v\left(w_{x}^{\tilde{d o w n}}\right)}{2} \\
S_{x}=\frac{A v\left(\tilde{w_{x}^{u p}}\right)-A v\left(w_{x}^{\tilde{d} w n}\right)-2 * w_{\text {earth }}}{2 * w_{\text {earth }}}
\end{gathered}
$$

In the similar way accelerometer errors can be determined by the following equations:

$$
\begin{gathered}
B_{x}=\frac{A v\left(\tilde{a_{x}^{u p}}\right)+A v\left(\tilde{a_{x}^{\text {down }}}\right)}{2} \\
S_{x}=\frac{A v\left(\tilde{a_{x}^{u p}}\right)-A v\left(\tilde{a_{x}^{\text {down }}}\right)-2 * g}{2 * g}
\end{gathered}
$$

Basic processing is simple but gyroscope and accelerometer misalignment errors cannot be calculated by basic processing. The Least Squares Fitting Method is used to overcome this problem and the LSF method is explained in the following part :

\section{Least Squares Fitting Method:}

All error parameters are estimated at the same time by using error equations (4.4) and (4.6). in matrix form. Least squares fitting method implementation for accelerometers is shown below: 


$$
P_{a c c}=M_{a c c} T_{a c c}{ }^{T}\left(T_{a c c} T_{a c c}{ }^{T}\right)^{-1}
$$

$P_{a c c}=\left[\begin{array}{cccc}1+S_{x} & M_{x y} & M_{x z} & B_{x} \\ M_{y x} & 1+S_{y} & M_{y z} & B_{y} \\ M_{z x} & M_{z y} & 1+S_{z} & B_{z}\end{array}\right]$, accelerometer error parameter matrix

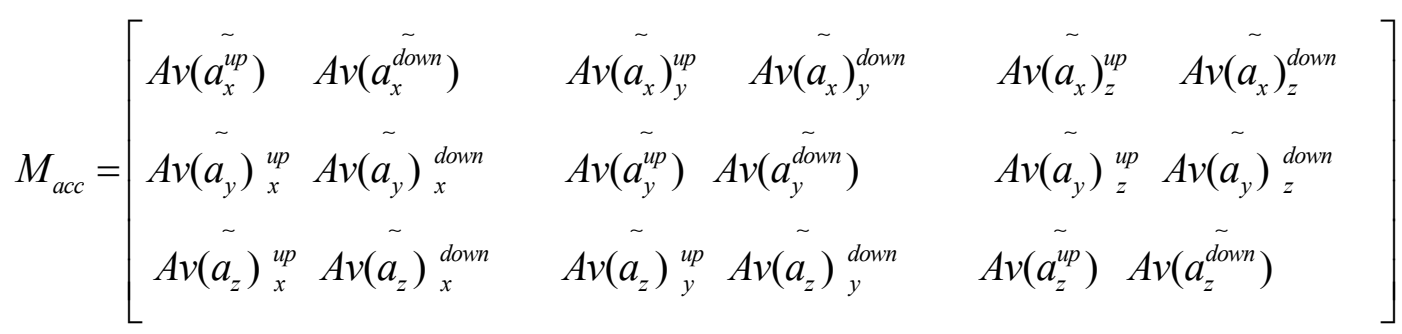

accelerometer measurement matrix

$T_{a c c}=\left[\begin{array}{rrrrrr}g & -g & 0 & 0 & 0 & 0 \\ 0 & 0 & g & -g & 0 & 0 \\ 0 & 0 & 0 & 0 & g & -g \\ 1 & 1 & 1 & 1 & 1 & 1\end{array}\right] \quad$, reference input matrix (local gravity)

Least squares fitting method implementation for gyroscopes is shown below:

$$
\begin{aligned}
& P_{g y r}=M_{g y r} T_{g y r}{ }^{T}\left(T_{g y r} T_{g y r}{ }^{T}\right)^{-1} \\
& P_{g y r}=\left[\begin{array}{cccc}
1+S_{x} & M_{x y} & M_{x z} & B_{x} \\
M_{y x} & 1+S_{y} & M_{y z} & B_{y} \\
M_{z x} & M_{z y} & 1+S_{z} & B_{z}
\end{array}\right] \quad \text {, gyroscope error parameter matrix }
\end{aligned}
$$

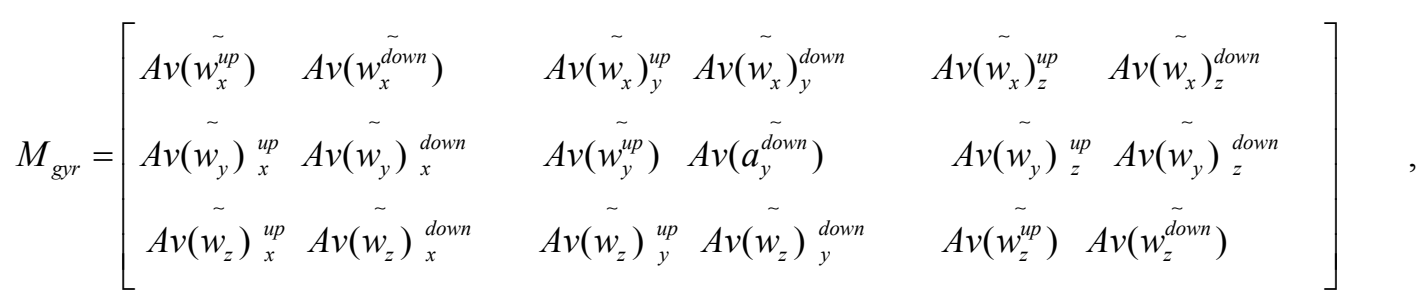

gyroscope measurement matrix 
$T_{g y r}=\left[\begin{array}{cccccl}w_{\text {earth }} & -w_{\text {earth }} & 0 & 0 & 0 & 0 \\ 0 & 0 & w_{\text {earth }} & -w_{\text {earth }} & 0 & 0 \\ 0 & 0 & 0 & 0 & w_{\text {earth }} & -w_{\text {earth }} \\ 1 & 1 & 1 & 1 & 1 & 1\end{array}\right] \quad$, reference input matrix (Earth's rotational rate)

\subsubsection{Rate Tests}

Rate tests are performed to extract the scale factor error, misalignment error and bias of lower grade gyroscopes. Performing rate tests require special test equipments such as rate table and flight motion simulator.

The procedure of rate tests is given in the following steps :

First step : The IMU is mounted on a rate table with mounting fixture.

Second step: The gyroscope data is collected and recorded when the rate table is rotated according to reference inputs in the sensitive axes $\mathrm{x}, \mathrm{y}$ and $\mathrm{z}$.

The collected rate test data can be processed by least squares fitting method and gyroscope error parameters can be extracted.

\subsubsection{Multi-Position Static Test}

This test method was developed and implemented within the scope of this thesis work. Multi-position test is an improved version of six-position static test and this test is performed to extract accelerometer scale factor error, misalignment and bias. In addition to that gyroscope bias and g-dependent bias coefficients can be extracted by using multi-position static tests.

IMU is placed on the rate table or flight motion simulator with a fixture. The accelerometer and gyroscope data is collected about 3 axis at 27 different position for 3 seconds. Local gravity is the reference input in this tests and the magnitude of acceleration which is sensed by the accelerometer is changed in each position by 
changing the axis position relative to local gravity. The angle between the measurement axis and the gravity vector is changed with 22.5 degree. Therefore the components of gravity vector can be observed on the other axes. This test method supplies to scan $1 \mathrm{~g}$ to $-1 \mathrm{~g}$ acceleration range with more input. In this way estimated error parameters which are close to actual error parameters can be obtained. Furthermore g-dependent bias coefficients of gyroscopes can be determined more accurately.

IMU is turned about $\mathrm{z}$ axis when $\mathrm{x}$ axis multi position static test is performed. Similarly, IMU is turned around $\mathrm{x}$ axis when $\mathrm{y}$ axis multi position static test is performed and, IMU is turned about/around y axis when $\mathrm{z}$ axis multi position static test is performed.

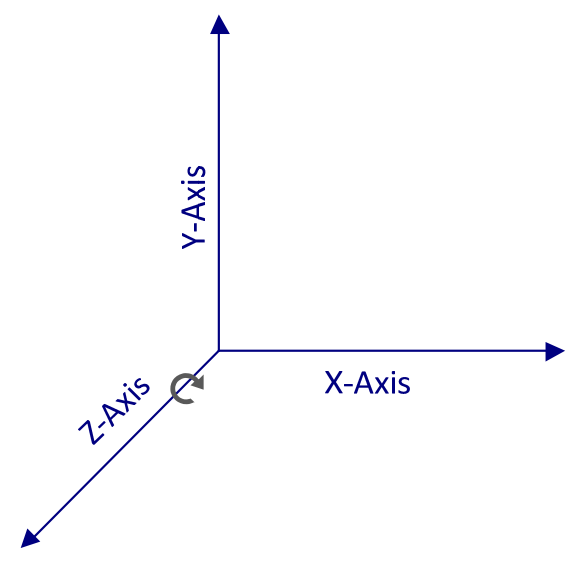

Figure 27 Simulated IMU measurement axis configuration
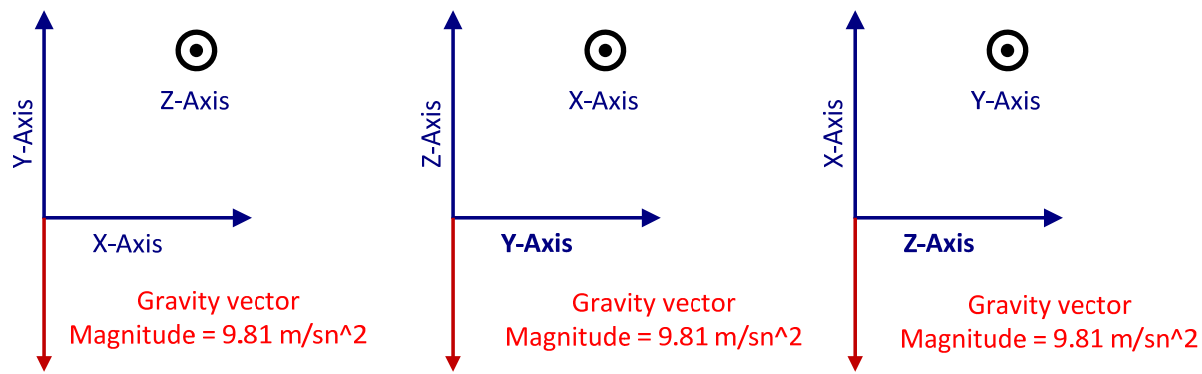

Figure 28 Multi-Position Test Configurations 
Figure 29 visualizes the axial motions by showing the test procedure. Figure 29 represents the test which is performed around $\mathrm{z}$ axis. This test is performed around all three axis to scan all accelerometers and gyroscopes at all positions.

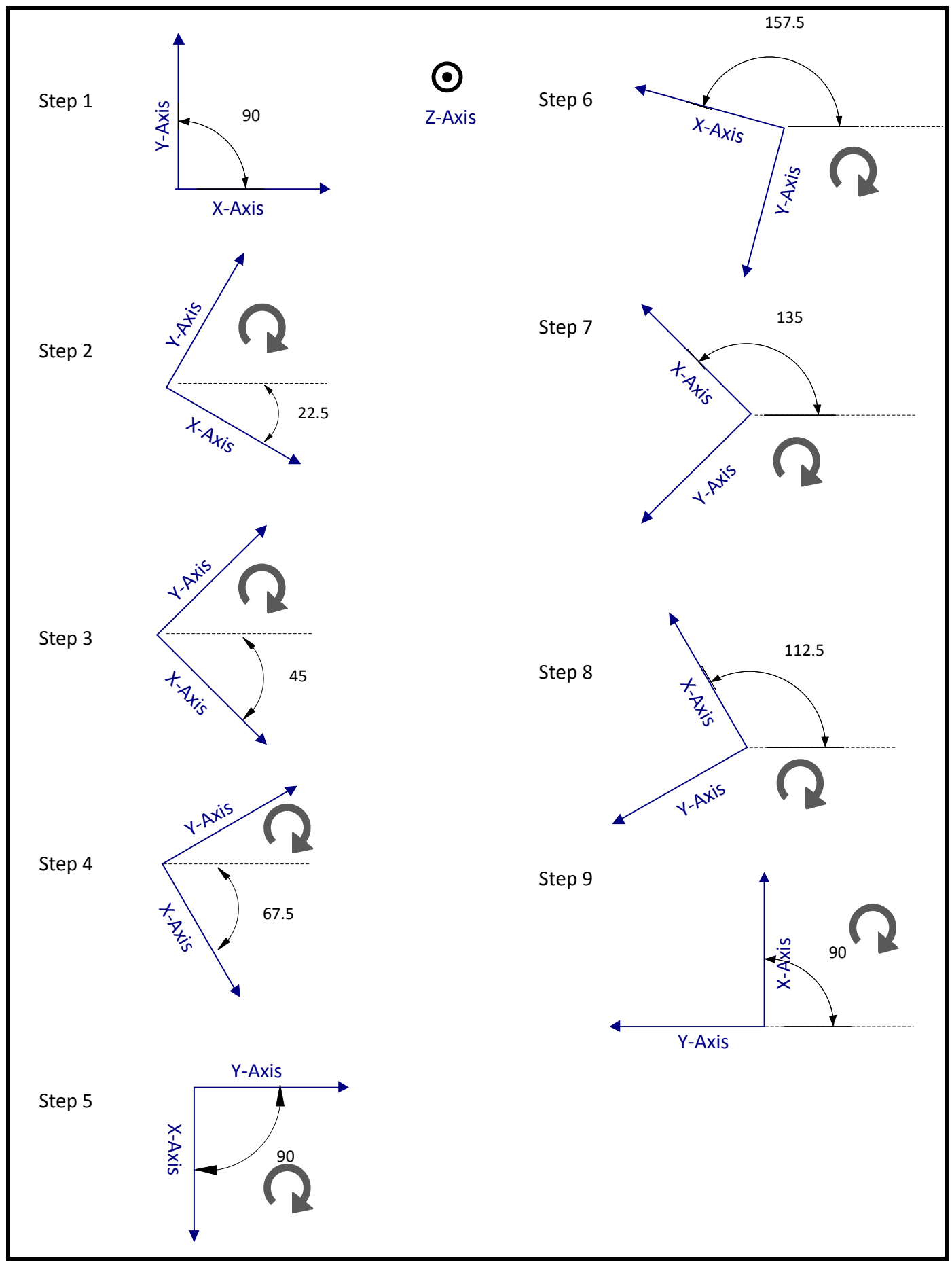

Figure 29 Multi Position Test Procedure 


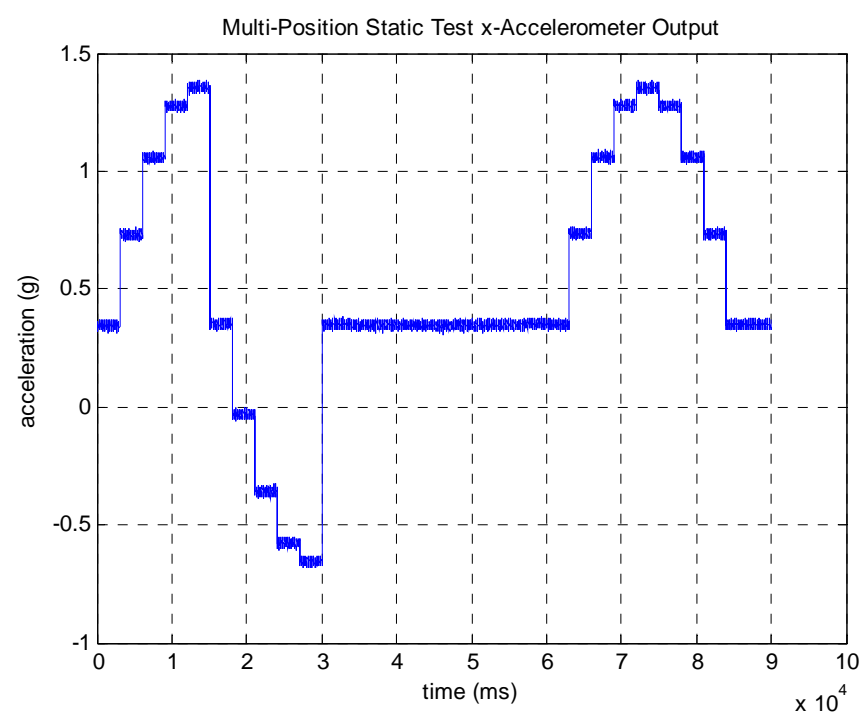

Figure 30 Multi-position static test $x$ acc output

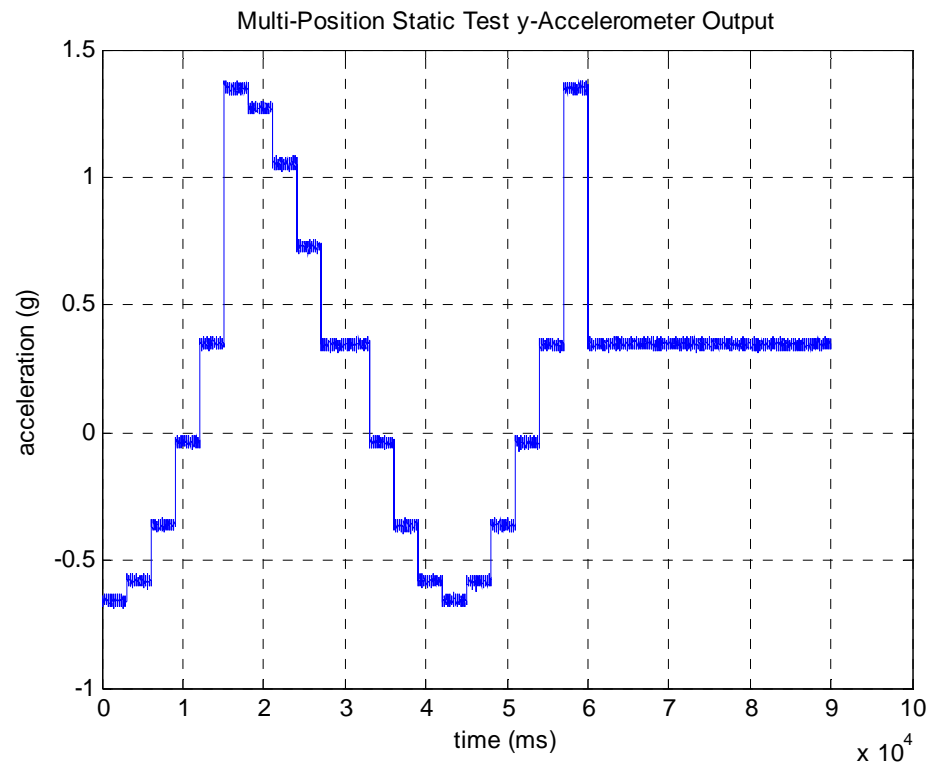

Figure 31 Multi-position static test y acc output 


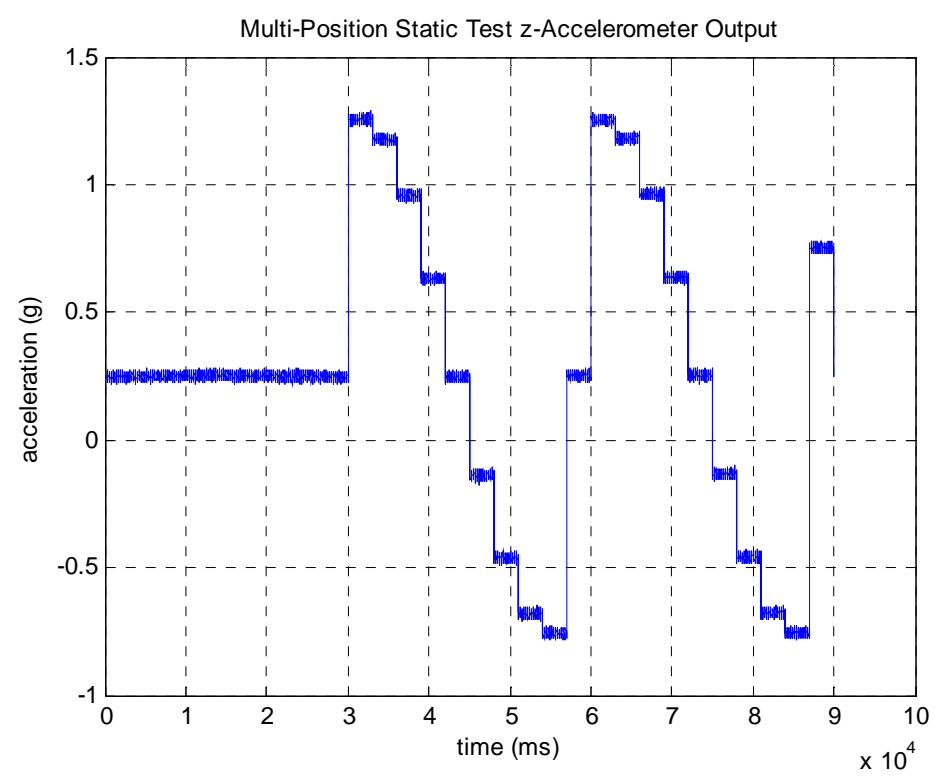

Figure 32 Multi-position static test z acc output

Figures 30 to 32 show the accelerometer measurements which are observed during the test period.

\subsubsection{Multi-Rate Dynamic Test}

This test method was developed and implemented within the scope of this thesis work. This rest method is performed to extract gyroscope scale factor error and misalignment.

Like multi-position static test, special test equipments like rate table, FMS are required to perform multi-rate dynamic test. IMU is placed on the rate table or flight motion simulator with a fixture. 3 axis gyroscope data is collected during the test period. Each axis test procedure has 20 different states. All states represent different angular rate. These states include 10 positive and 10 negative angular rate and vary between 0 and $200 \mathrm{deg} / \mathrm{s}$. Figure 33,34 and 35 present multi-rate dynamic test gyroscope measurements. 


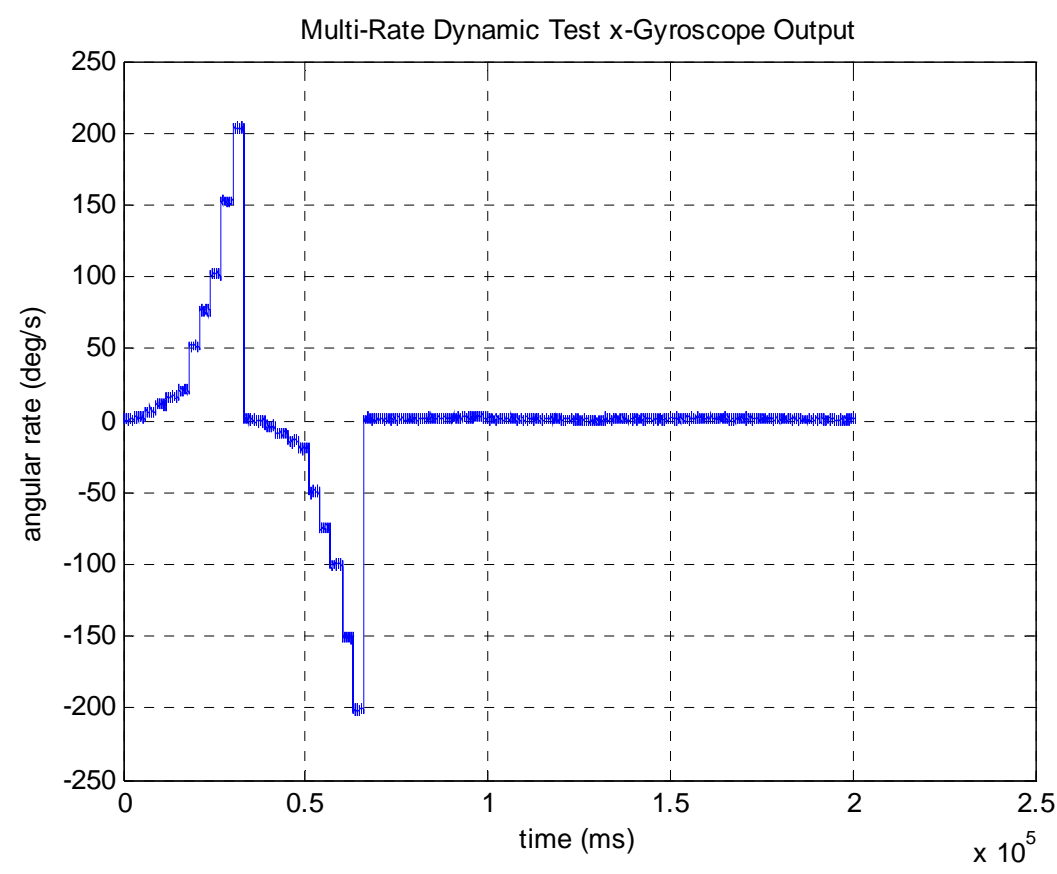

Figure 33 Multi-rate dynamic test $x$ gyro output

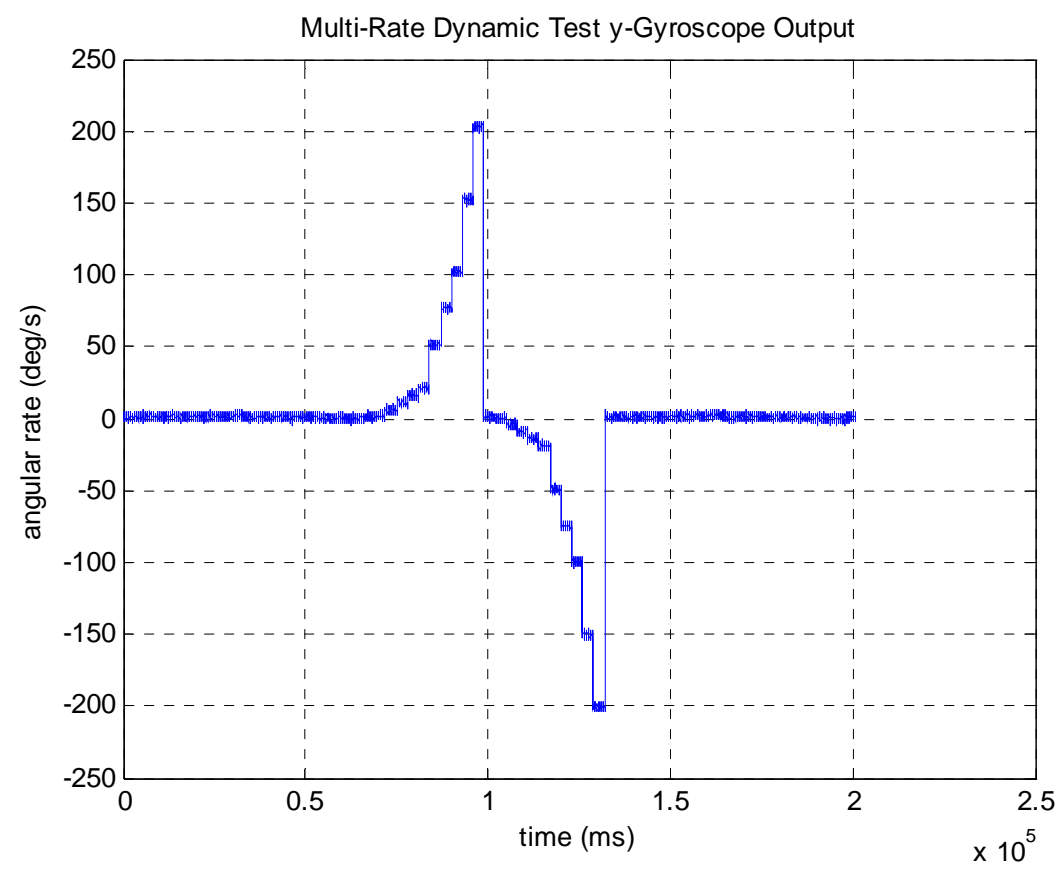

Figure 34 Multi-rate dynamic test y gyro output 


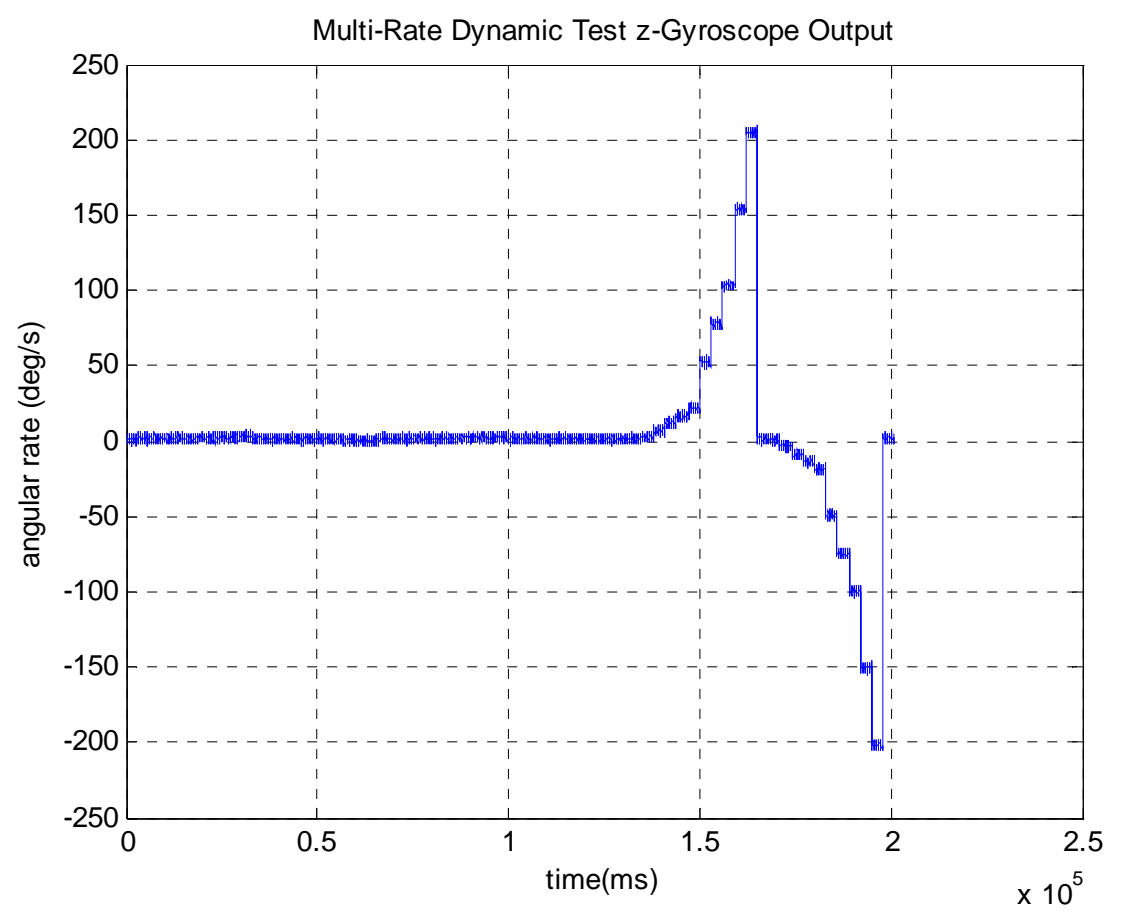

Figure 35 Multi-rate dynamic test z gyro output

\subsubsection{Data Processing Algorithm for Multi-Position Static and Multi-Rate Dynamic Tests}

IMU complete calibration test which is used in this thesis work consists of multiposition static test and multi-rate dynamic test. The complete calibration test procedure is summarized in the following paragraph:

An IMU is mounted on 3 axis flight motion simulator with a fixture. Multi-position static test and multi-rate dynamic test are performed. Data is collected at each position and each rate for 3 seconds. (Sampling rate is $1 \mathrm{kHz}$ and approximately 3000 data points are collected in each step).

The collected data is processed by using deterministic error estimation codes.

Processing steps are explained below:

First step : Decomposing position states and angular rates from test data 
Second step : Taking the average value of each position and rate.

Third step : Creating the true and measurement matrices for least square fitting. (Each measurement has equal weight to calculate calibration parameters)

Fourth step : Implementing least square fitting and calculating error (calibration) parameters.

Deterministic error estimation codes were developed by using MATLAB software.

Deterministic error codes depend on least squares fitting method implementation. Multi position test data is used to determine accelerometer bias, scale factor error and misalignment parameters. Additionally, gyroscope bias and g-dependent bias parameters are also extracted from multi-position static test data.

Least squares fitting formulation for accelerometer is similar with equation (5.5). Contents of the matrices are updated according to multi-position static test states. Matrices, which are updated according to the states are given below.

$P_{a c c}=M_{a c c} T_{a c c}{ }^{T}\left(T_{a c c} T_{a c c}{ }^{T}\right)^{-1} \quad$ (for acc bias, misalignment and scale factor)

$P_{a c c}=\left[\begin{array}{cccc}1+S_{x} & M_{x y} & M_{x z} & B_{x} \\ M_{y x} & 1+S_{y} & M_{y z} & B_{y} \\ M_{z x} & M_{z y} & 1+S_{z} & B_{z}\end{array}\right]$, matrix of estimated accelerometer error parameters (this matrix is taken from equation (5.5))

Reference input matrix and measured data matrices are also updated according to multi-position static test states. These matrices are given in the following equations. 


\begin{tabular}{|c|c|c|c|c|}
\hline \multirow{3}{*}{\multicolumn{2}{|c|}{$\left[\begin{array}{l}g * \cos (270) \\
g * \cos (292.5) \\
g * \cos (315)\end{array}\right.$}} & $g * \cos (180)$ & 0 & 1 \\
\hline & & $g * \cos (202.5)$ & 0 & 1 \\
\hline & & $g * \cos (225)$ & 0 & 1 \\
\hline \multirow{24}{*}{$T_{a c c}=$} & $g * \cos (337.5)$ & $g * \cos (247.5)$ & 0 & 1 \\
\hline & $g^{*} \cos (0)$ & $g * \cos (270)$ & 0 & 1 \\
\hline & $g * \cos (112.5)$ & $g * \cos (22.5)$ & 0 & 1 \\
\hline & $g * \cos (135)$ & $g * \cos (45)$ & 0 & 1 \\
\hline & $g * \cos (157.5)$ & $g * \cos (67.5)$ & 0 & 1 \\
\hline & $g * \cos (180)$ & $g * \cos (270)$ & 0 & 1 \\
\hline & 0 & $g * \cos (270)$ & $g * \cos (0)$ & 1 \\
\hline & 0 & $g * \cos (247.5)$ & $g * \cos (22.5)$ & 1 \\
\hline & 0 & $g * \cos (225)$ & $g * \cos (45)$ & 1 \\
\hline & 0 & $g * \cos (202.5)$ & $g * \cos (67.5)$ & 1 \\
\hline & 0 & $g * \cos (180)$ & $g * \cos (90)$ & 1 \\
\hline & 0 & $g * \cos (157.5)$ & $g * \cos (112.5)$ & 1 \\
\hline & 0 & $g * \cos (135)$ & $g * \cos (135)$ & 1 \\
\hline & 0 & $g * \cos (112.5)$ & $g * \cos (157.5)$ & 1 \\
\hline & 0 & $g * \cos (90)$ & $g^{*} \cos (180)$ & 1 \\
\hline & $g * \cos (270)$ & 0 & $g^{*} \cos (0)$ & 1 \\
\hline & $g * \cos (292.5)$ & 0 & $g * \cos (22.5)$ & 1 \\
\hline & $g * \cos (315)$ & 0 & $g * \cos (45)$ & 1 \\
\hline & $g * \cos (337.5)$ & 0 & $g * \cos (67.5)$ & 1 \\
\hline & $g * \cos (360)$ & 0 & $g * \cos (90)$ & 1 \\
\hline & $g^{*} \cos (22.5)$ & 0 & $g * \cos (112.5)$ & 1 \\
\hline & $g * \cos (45)$ & 0 & $g * \cos (135)$ & 1 \\
\hline & $g * \cos (67.5)$ & 0 & $g * \cos (157.5)$ & 1 \\
\hline & $g * \cos (90)$ & 0 & $g * \cos (180)$ & 1 \\
\hline
\end{tabular}

Equation (5.7) represents the accelerometer reference inputs and the following equation, (5.8) gives the average value of accelerometer outputs. 


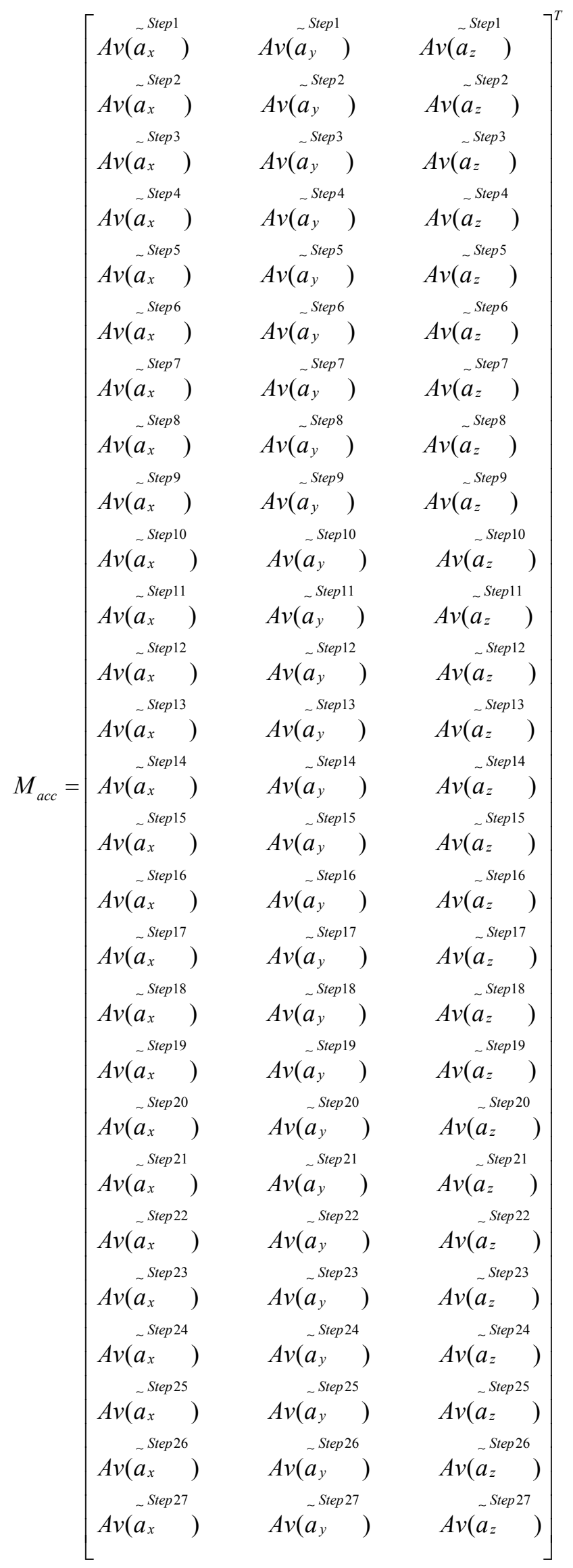


Equation (5.6) was arranged to adapt least square fitting method to multi position test states. Gyroscope data, which is collected during the multi-position static test is used to determine gyroscope bias and g-dependent bias.

It is assumed that there is not any angular rate input at any static position. Therefore, gyroscope outputs contain only bias and g-dependent bias effects and bias and gdependent bias coefficients can be acquired from multi-position test gyroscope outputs.

Reference input matrix is identical with the accelerometer reference input matrix.

$P_{g y r}=M_{g y r} T_{g y r}{ }^{T}\left(T_{g y r} T_{g y r}{ }^{T}\right)^{-1} \quad, \quad P_{g y r}=\left[\begin{array}{cccc}B_{g x} & 0 & 0 & B_{x} \\ 0 & B_{g y} & 0 & B_{y} \\ 0 & 0 & B_{g z} & B_{z}\end{array}\right] \quad, \quad T_{g y r}=T_{a c c}$

Updated measurement matrix is given in equation (5.9). 


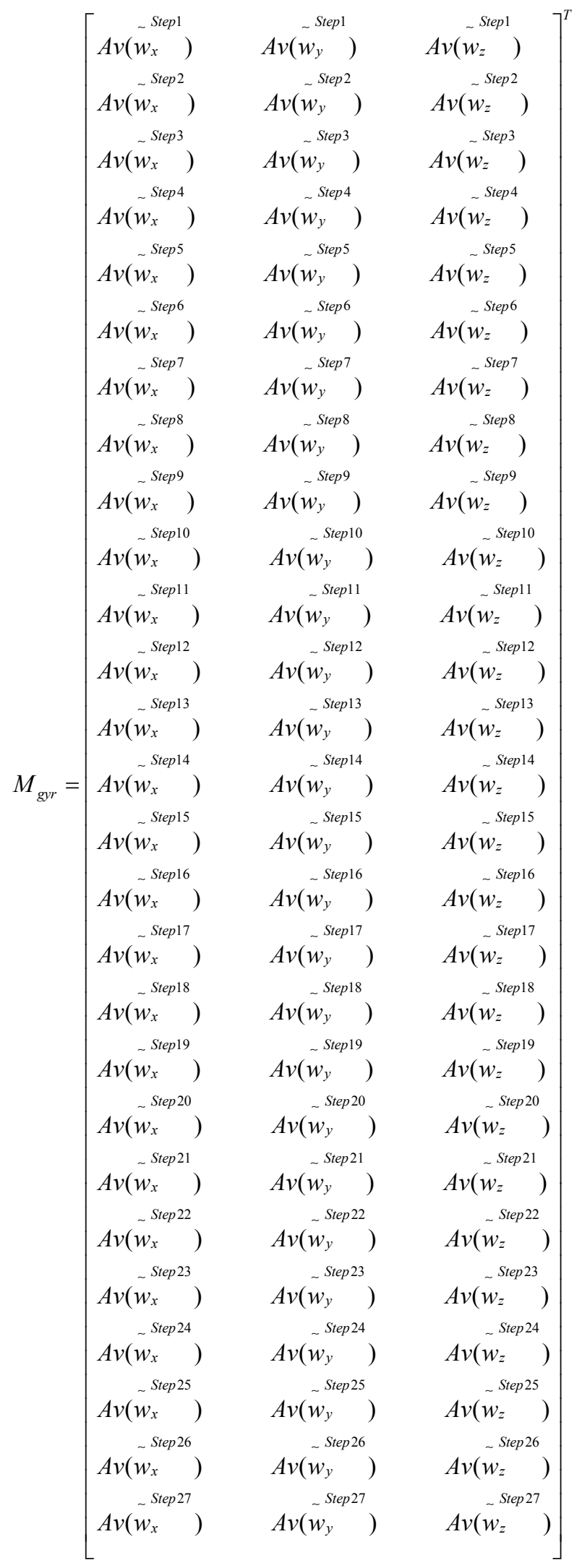


Multi rate dynamic test data is used to determine gyroscope scale factor error and misalignment parameters. In order to extract gyroscope scale factor and misalignment equation (5.6) is arranged according to multi-rate dynamic test states.

$$
P_{g y r}=M_{g y r} T_{g y r}{ }^{T}\left(T_{g y r} T_{g y r}{ }^{T}\right)^{-1} \quad, \quad P_{g y r}=\left[\begin{array}{cccc}
1+S_{x} & M_{x y} & M_{x z} & B_{x} \\
M_{y x} & 1+S_{y} & M_{y z} & B_{y} \\
M_{z x} & M_{z y} & 1+S_{z} & B_{z}
\end{array}\right]
$$

$$
\left.T_{g y r_{x}}=\left[\begin{array}{rrr}
001 & 0 & 0 \\
005 & 0 & 0 \\
010 & 0 & 0 \\
015 & 0 & 0 \\
020 & 0 & 0 \\
050 & 0 & 0 \\
075 & 0 & 0 \\
100 & 0 & 0 \\
150 & 0 & 0 \\
-001 & 0 & 0 \\
-005 & 0 & 0 \\
-010 & 0 & 0 \\
-015 & 0 & 0 \\
-020 & 0 & 0 \\
-050 & 0 & 0 \\
-075 & 0 & 0 \\
-100 & 0 & 0 \\
-150 & 0 & 0 \\
-200 & 0 & 0
\end{array}\right]^{T}\right]_{g y r_{y}}=\left[\begin{array}{rrr}
0 & 001 & 0 \\
0 & 005 & 0 \\
0 & 010 & 0 \\
0 & 015 & 0 \\
0 & 020 & 0 \\
0 & 050 & 0 \\
0 & 075 & 0 \\
0 & 100 & 0 \\
0 & 150 & 0 \\
0 & 200 & 0 \\
0 & -005 & 0 \\
0 & -010 & 0 \\
0 & -015 & 0 \\
0 & -020 & 0 \\
0 & -050 & 0 \\
0 & -075 & 0 \\
0 & -100 & 0 \\
0 & -150 & 0 \\
0 & -200 & 0
\end{array}\right]^{T}
$$




$$
\left.T_{g y r_{z}}=\left[\begin{array}{rrr}
0 & 0 & 001 \\
0 & 0 & 005 \\
0 & 0 & 010 \\
0 & 0 & 015 \\
0 & 0 & 020 \\
0 & 0 & 050 \\
0 & 0 & 075 \\
0 & 0 & 100 \\
0 & 0 & 150 \\
0 & 0 & 200 \\
0 & 0 & -001 \\
0 & 0 & -010 \\
0 & 0 & -015 \\
0 & 0 & -020 \\
0 & 0 & -050 \\
0 & 0 & -075 \\
0 & 0 & -100 \\
0 & 0 & -150 \\
0 & 0 & -200
\end{array}\right]_{g y r_{y}} T_{g y r_{z}}\right]
$$




\begin{tabular}{|c|c|c|c|}
\hline & $\sim 001$ & ح001 & $\sim$ ح001 \\
\hline & $A v\left(w_{x}\right)$ & $A v\left(w_{y}\right)$ & $A v\left(w_{z}\right)$ \\
\hline & $\sim 005$ & $\sim x 005$ & $\sim 005$ \\
\hline & $A v\left(w_{x}\right)$ & $A v(w y \quad)$ & $A v\left(w_{z}\right)$ \\
\hline & $\sim$ $>10$ & $\sim x 010$ & $\sim x 010$ \\
\hline & $A v\left(w_{x}\right)$ & $A v\left(w_{y}\right)$ & $A v\left(w_{z}\right)$ \\
\hline & ح015 & $\sim 015$ & $\sim 015$ \\
\hline & $A v\left(w_{x}\right)$ & $A v\left(w_{y}\right)$ & $A v\left(w_{z}\right)$ \\
\hline & $\sim x 020$ & $\sim x 020$ & $\sim x 020$ \\
\hline & $A v\left(w_{x}\right)$ & $A v\left(w_{y} \quad\right)$ & $A v\left(w_{z}\right)$ \\
\hline & $\sim \times 050$ & $\sim \times 050$ & $\sim x 050$ \\
\hline & $A v\left(w_{x} \quad\right)$ & $A v\left(w_{y}\right)$ & $A v\left(w_{z}\right)$ \\
\hline & $\sim 075$ & $\sim \times 075$ & $\sim$ x075 \\
\hline & $A v\left(w_{x}\right)$ & $A v\left(w_{y}\right)$ & $A v\left(w_{z}\right)$ \\
\hline & $\sim x 100$ & $\sim x 100$ & $\sim x 100$ \\
\hline & $A v\left(w_{x}\right)$ & $A v\left(w_{y}\right)$ & $A v\left(w_{z} \quad\right)$ \\
\hline & $\sim \times 150$ & $\sim x 150$ & $\sim$ x150 \\
\hline & $A v\left(w_{x}\right)$ & $A v\left(w_{y}\right)$ & $A v\left(w_{z}\right)$ \\
\hline & $\sim x 200$ & $\sim x 200$ & $\sim x 200$ \\
\hline & $A v\left(w_{x}\right)$ & $A v\left(w_{y}\right)$ & $A v\left(w_{z}\right)$ \\
\hline$V_{g} g p_{x}-$ & $\sim-x 001$ & $\sim-x 001$ & $\sim-x 001$ \\
\hline & $A v\left(w_{x} \quad\right)$ & $A v\left(w_{y} \quad\right)$ & $A v\left(w_{z} \quad\right)$ \\
\hline & $\sim^{-x 005}$ & $\sim-x 005$ & $\sim \sim^{-x 005}$ \\
\hline & $A v\left(w_{x}\right)$ & $A v\left(w_{y} \quad\right)$ & $A v\left(w_{z}\right)$ \\
\hline & $\sim^{-x 010}$ & $\sim^{-x 010}$ & $\sim^{-x 010}$ \\
\hline & $A v\left(w_{x} \quad\right)$ & $A v\left(w_{y} \quad\right)$ & $A v\left(w_{z} \quad\right)$ \\
\hline & $\sim^{-x 015}$ & $\sim^{-x 015}$ & $\sim^{-x 015}$ \\
\hline & $A v\left(w_{x} \quad\right)$ & $A v\left(w_{y} \quad\right)$ & $A v\left(w_{z} \quad\right)$ \\
\hline & $\sim^{-x 020}$ & $\sim^{-x 020}$ & $\sim^{-x 020}$ \\
\hline & $A v\left(w_{x}\right)$ & $A v\left(w_{y} \quad\right)$ & $A v\left(w_{z} \quad\right)$ \\
\hline & $\sim^{-x 050}$ & $\sim-x 050$ & $\sim^{-x 050}$ \\
\hline & $A v\left(w_{x}\right)$ & $A v\left(w_{y}\right)$ & $A v\left(w_{z}\right)$ \\
\hline & $\sim^{-x 075}$ & $\sim^{-x 075}$ & $\sim^{-x 075}$ \\
\hline & $A v\left(w_{x}\right)$ & $A v\left(w_{y} \quad\right)$ & $A v\left(w_{z} \quad\right)$ \\
\hline & $\sim-x 100$ & $\sim-x 100$ & $\sim^{-x 100}$ \\
\hline & $A v\left(w_{x}\right)$ & $A v\left(w_{y} \quad\right)$ & $A v\left(w_{z} \quad\right)$ \\
\hline & $\sim-x 150$ & $\sim-x 150$ & $\sim-x 150$ \\
\hline & $A v\left(w_{x}\right)$ & $A v\left(w_{y}\right)$ & $A v\left(w_{z} \quad\right)$ \\
\hline & $\sim^{-x 200}$ & $\sim-x 200$ & $\sim^{-x 200}$ \\
\hline & $A v\left(w_{x} \quad\right)$ & $A v\left(w_{y} \quad\right)$ & $\left.A v\left(w_{z} \quad\right)\right]$ \\
\hline
\end{tabular}




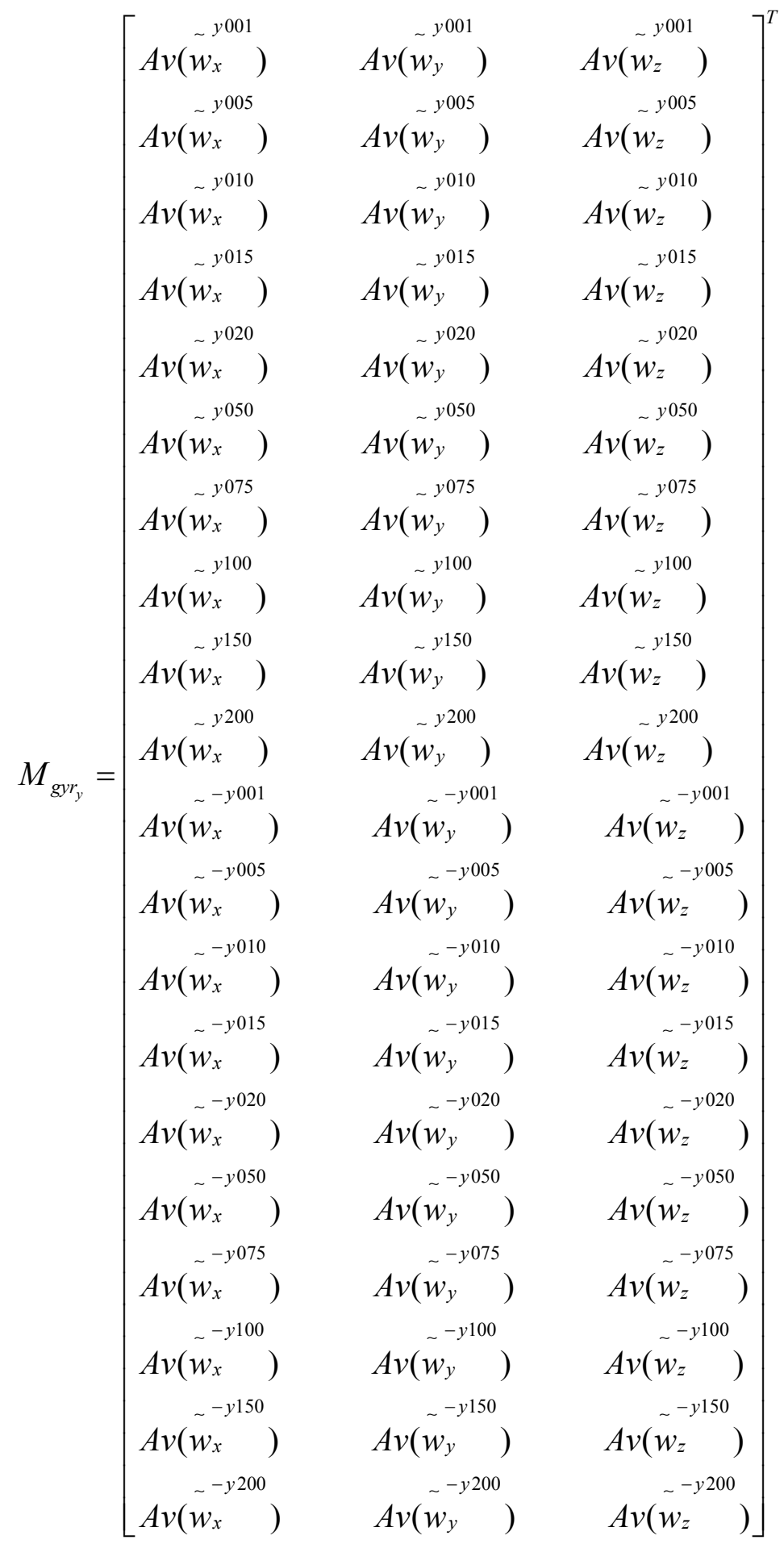




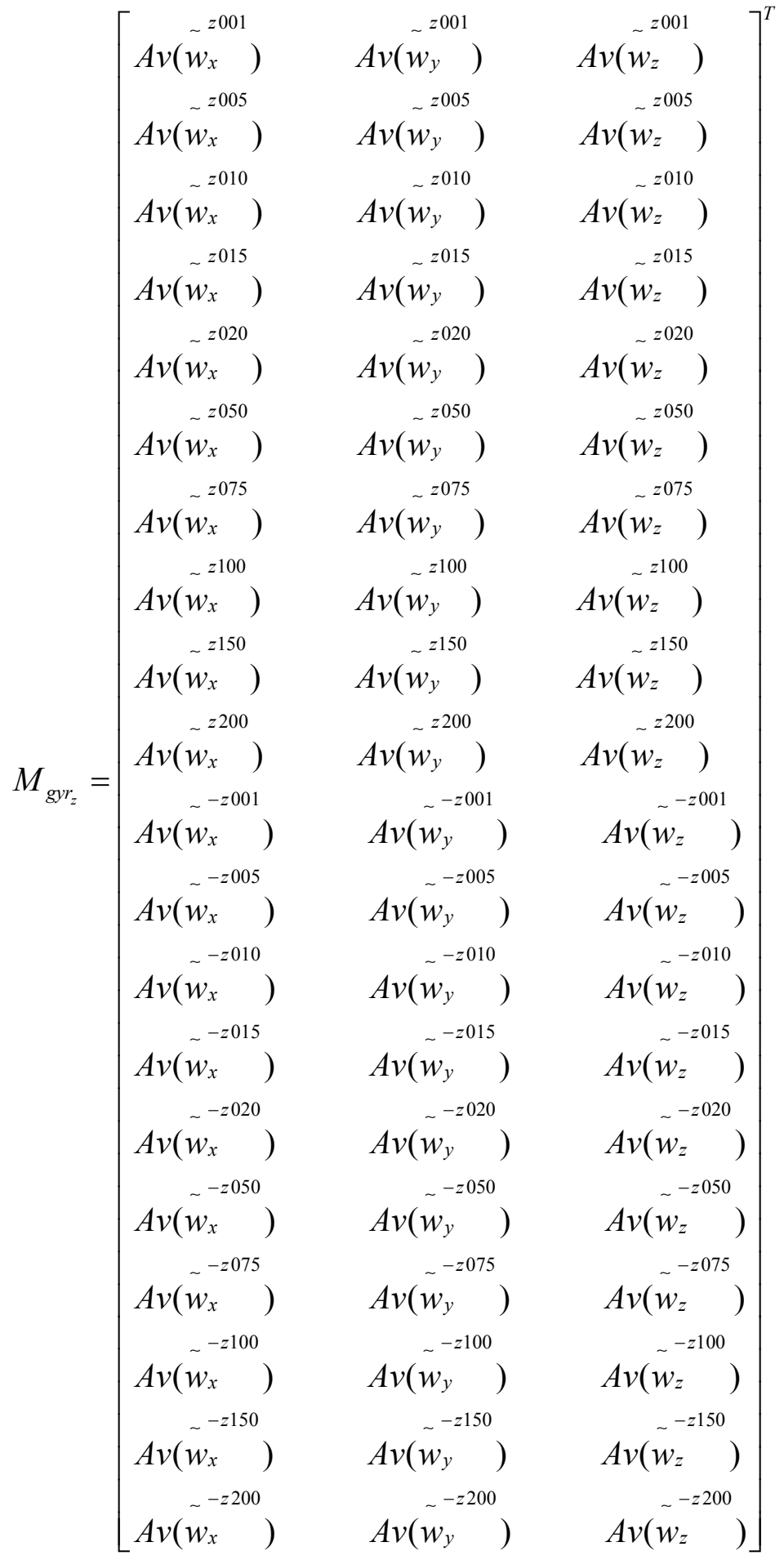

$$
\begin{aligned}
& M_{g y r}=\left[\begin{array}{lll}
M_{g y r_{x}} & M_{g y r_{y}} & M_{g y r_{z}}
\end{array}\right]
\end{aligned}
$$




\subsection{Deterministic Error Compensation Model}

The aim of deterministic error compensation algorithm is compensating the effect of deterministic error parameters of an IMU. A deterministic error compensation algorithm was developed and implemented within the scope of this thesis work. Output of the compensation model was used as input of the stochastic error estimation algorithm.

The error model of the single axis accelerometer and gyroscope were given in equation (4.1) and (4.2). IMU deterministic error compensation algorithms are based on inverse of error models.

$$
\hat{a}_{x}=\left(1+S_{x}\right)^{-1}\left(\tilde{a}_{x}-B_{x}\right)
$$

$\tilde{a}_{x}-\hat{a}_{x}=$ residual accelerometer error $=$ accelerometer stochastic error

$$
\hat{w}_{x}=\left(1+S_{x}\right)^{-1}\left(\tilde{a}_{x}-B_{x}-B_{g x} a_{x}\right)
$$

$\tilde{w}_{x}-\hat{w}_{x}=$ residual gyroscope error $=$ gyroscope stochastic error

$$
\begin{aligned}
& {\left[\begin{array}{c}
\hat{a_{x}} \\
\hat{a_{y}} \\
\hat{a}_{z}
\end{array}\right]=\left[\begin{array}{ccc}
1+S_{x} & M_{x y} & M_{x z} \\
M_{y x} & 1+S_{y} & M_{y z} \\
M_{z x} & M_{z y} & 1+S_{z}
\end{array}\right]^{-1}\left[\begin{array}{c}
\tilde{a_{x}}-B_{x} \\
\tilde{a_{y}}-B_{y} \\
\tilde{a}_{z}-B_{z}
\end{array}\right]} \\
& \left.\left[\begin{array}{c}
\hat{w_{x}} \\
\hat{w_{y}} \\
\hat{w_{z}}
\end{array}\right]=\left[\begin{array}{ccc}
1+S_{x} & M_{x y} & M_{x z} \\
M_{y x} & 1+S_{y} & M_{y z} \\
M_{z x} & M_{z y} & 1+S_{z}
\end{array}\right]\left[\begin{array}{c}
\tilde{w}_{x}-B_{x} \\
\tilde{w}_{y}-B_{y} \\
\tilde{w}_{z}-B_{z}
\end{array}\right]-\left[\begin{array}{ccc}
B_{g x} & 0 & 0 \\
0 & B_{g y} & 0 \\
0 & 0 & B_{g z}
\end{array}\right]\left[\begin{array}{c}
\hat{a_{x}} \\
\hat{a_{y}} \\
\hat{a}_{z}
\end{array}\right]\right]
\end{aligned}
$$


Deterministic error compensation simulation model was developed by using MATLAB Simulink software. Equation (5.17) and (5.18) were generated in Simulink model. The error compensation model was used to process collected calibration data to observe true measurement values. Sensor error parameters were extracted by the deterministic error estimation codes and the extracted parameters were embedded to the deterministic error compensation model.

In real applications, error compensation algorithms are designed according to the types of sensors and converted to embedded software. Then this software is installed on the IMU processor. 


\section{ESTIMATION OF STOCHASTIC ERROR PARAMETERS}

This chapter suggests a method for estimation of stochastic sensor error parameters. The Kalman Filter algorithm which is explained in Chapter 3, was used as estimation technique. This technique needs a system model and a measurement model. Therefore, Section 6.1 explains the system model and Section 6.2 gives the measurement model properties.

\subsection{System Model}

As mentioned in Chapter 4 gyroscope and accelerometer bias instabilities are modeled with first order Gauss-Markov process. An IMU has three accelerometers and three gyroscopes for measuring linear acceleration and angular rate about $\mathrm{x}, \mathrm{y}$ and $z$ axis. Therefore six sensors' bias instabilities were estimated by using Kalman filter. For this reason, the state vector contains six states and these states represent the bias instability of $\mathrm{x}$ gyroscope, $\mathrm{y}$ gyroscope, $\mathrm{z}$ gyroscope, $\mathrm{x}$ accelerometer, $\mathrm{y}$ accelerometer and $\mathrm{z}$ accelerometer respectively. The bias instability model of an inertial sensor was given with equation (4.1). That equation was developed for six sensor (3 gyroscope and 3 accelerometer) implementation and developed system model is presented in equation (6.1). 


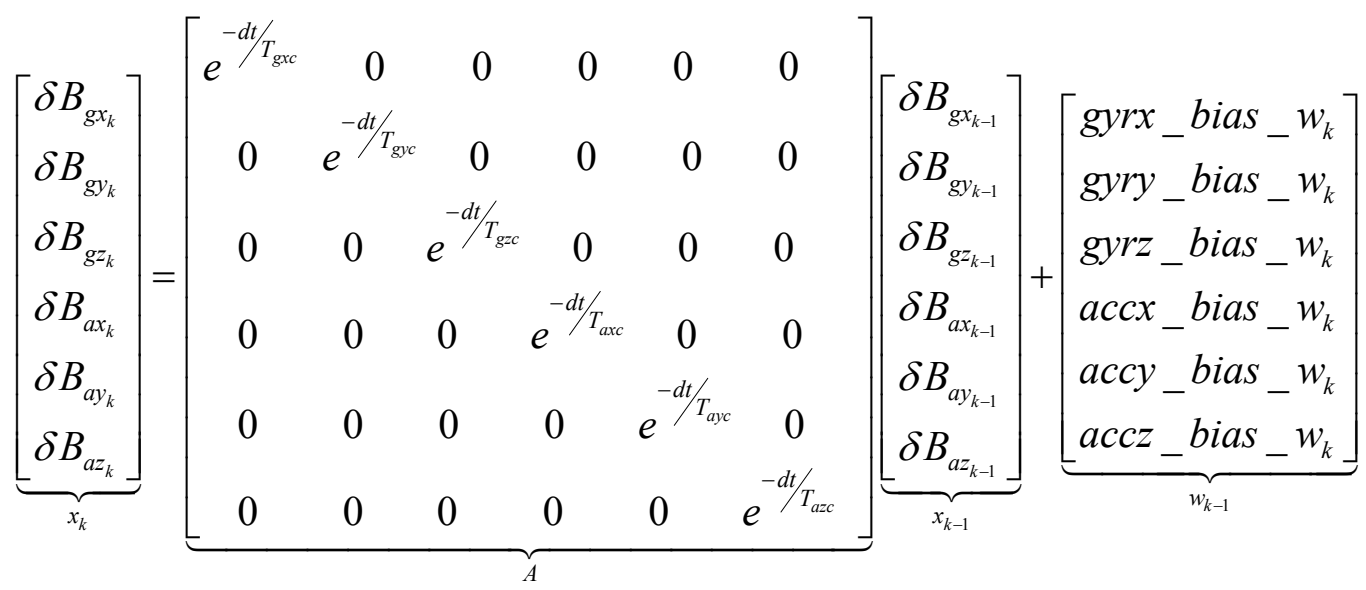

$\delta B_{g x_{k}}: x$ gyro instability at time $k, T_{g x c}: x$ gyro time constant $\delta B_{g y_{k}}: y$ gyro instability at time $k, T_{g y c}: y$ gyro time constant $\delta B_{g z_{k}}: z$ gyro instability at time $k, T_{g z c}: z$ gyro time constant $\delta B_{a x_{k}}: x$ acc instability at time $k, T_{a x c}: x$ acc time constant $\delta B_{a y_{k}}: x$ acc instability at time $k, T_{a y c}: y$ acc time constant $\delta B_{a z_{k}}: x$ acc instability at time $k, T_{a z c}: z$ acc time constant

$\delta B_{g_{k-1}}: x$ gyro instability at time $k-1, \quad$ gyrx_bias_ $w_{k}: x$ gyro driven noise $\delta B_{g y_{k-1}}: y$ gyro instability at time $k-1$, gyry_bias_ $w_{k}: y$ gyro driven noise $\delta B_{g z_{k-1}}: z$ gyro instability at time $k-1, \quad$ gyrz_bias_ $w_{k}: z$ gyro driven noise $\delta B_{a x_{k-1}}: x$ acc instability at time $k-1, \quad$ accx_bias_ $w_{k}: x$ acc driven noise $\delta B_{a y_{k-1}}: x$ acc instability at time $k-1$, accy_bias_ $w_{k}: y$ acc driven noise $\delta B_{a z_{k-1}}: x$ acc instability at time $k-1, \quad$ accz_bias_ $w_{k}: z$ acc driven noise

\subsection{Measurement Model}

The measurement model was constituted according to equation (3.3). Sensor measurements represent and random sensor noises represent the measurement noise vector. After the compensation of deterministic errors, gyroscope and accelerometer bias instabilities can be observed from sensor measurements directly. Therefore, measurement conversion matrix $C$ tuned as an identity matrix. 
The error compensation model's outputs were used as measurements in Kalman filter algorithm. Therefore measurement matrix was selected as an identity matrix.

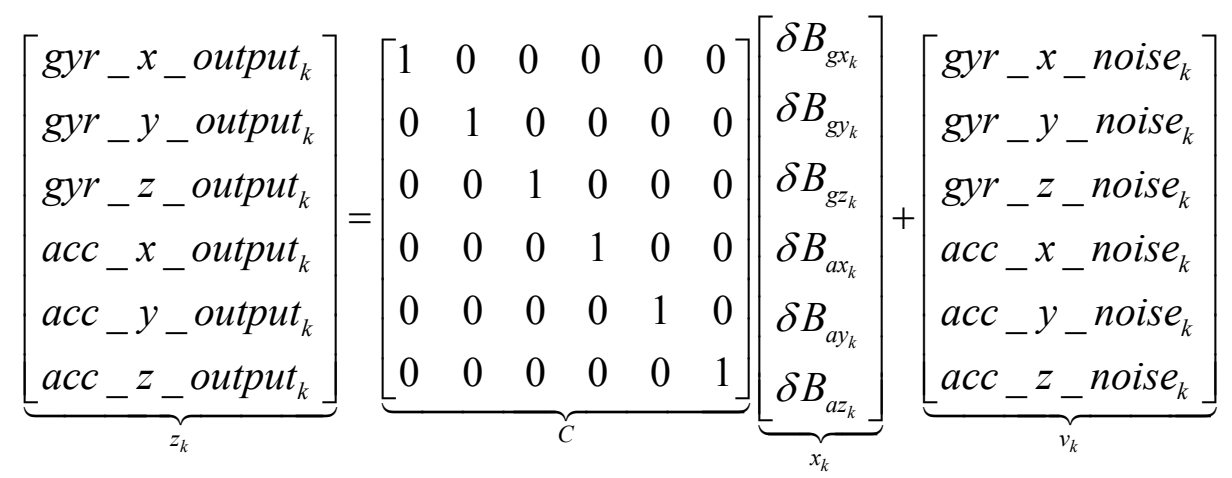

\subsection{Tuning of Kalman Filter Parameters}

All inertial sensors have some characteristic parameters such as time constant, random noise density and bias instability. In this thesis study these parameters are very useful inputs for Kalman filter parameter tuning and algorithm initialization. For example, gyroscope and accelerometer random noise densities were used to determine measurement noise covariance, $R$. Similarly, $3 \sigma$ value of gyroscope and accelerometer bias instabilities were used to constitute initial value of error covariance matrix, $P$.

\subsubsection{Error Covariance Matrix Initialization}

It was assumed that $\delta B_{g x_{k}}=\delta B_{g y_{k}}=\delta B_{g z_{k}}=\delta B_{a x_{k}}=\delta B_{a y_{k}}=\delta B_{a z_{k}}=0$ for $\mathrm{k}=0$.

As mentioned in Chapter 3, error covariance represents the uncertainty in the state estimate. The estimate error, uncertainty in the states estimate, and the simplest representation of error covariance is given in equation (6.3) and (6.4).

$$
\begin{gathered}
e_{k}=x_{k}-\hat{x_{k}} \\
x_{k} \text { :true state, } \quad \hat{x_{k}} \text { :estimated state }
\end{gathered}
$$




$$
P_{k}=E\left[e_{k} e_{k}^{T}\right]
$$

The maximum value of the true state is $3 \sigma$ value of bias instability. Therefore, if the initial states are equal to zero, on the basis of equation (6.4), the maximum value of error can be $3 \sigma$ value of bias instability. For this reason initial value of the error covariance $P_{k}$ 's diagonal elements become $(3 \sigma)^{2}$ value of gyroscope and accelerometer bias instabilities. There is no correlation between the states and errors. Therefore, the off diagonal elements of $P_{k}$ are equal to zero.

$$
P_{0}=\left[\begin{array}{cccccc}
\left(3 \sigma_{\delta B_{g x}}\right)^{2} & 0 & 0 & 0 & 0 & 0 \\
0 & \left(3 \sigma_{\delta B_{g y}}\right)^{2} & 0 & 0 & 0 & 0 \\
0 & 0 & \left(3 \sigma_{\delta B_{g z}}\right)^{2} & 0 & 0 & 0 \\
0 & 0 & 0 & \left(3 \sigma_{\delta B_{a x}}\right)^{2} & 0 & 0 \\
0 & 0 & 0 & 0 & \left(3 \sigma_{\delta B_{a y}}\right)^{2} & 0 \\
0 & 0 & 0 & 0 & 0 & \left(3 \sigma_{\delta B_{a z}}\right)^{2}
\end{array}\right]
$$

\subsubsection{Tuning of Measurement and Process Noise Covariance Matrix}

Like sensor bias instability, random sensor noise density is represented by $1 \sigma$ value. In simulations and calculations, the noise density is multiplied by square root of sampling frequency to convert the noise density to angular rate noise and acceleration noise. Similar to the error covariance initialization, $(3 \sigma)^{2}$ value of angular rate noise and acceleration noise were used to tuning of measurement noise covariance matrix diagonal elements. The $3 \sigma$ value of angular rate noise and acceleration noise denote the maximum values of the noise components. There is no correlation between measurements. Therefore, the off diagonal elements of $R$ are equal to zero. 


$$
R=\left[\begin{array}{cccccc}
\left(3 \sigma_{n_{g y x x}}\right)^{2} & 0 & 0 & 0 & 0 & 0 \\
0 & \left(3 \sigma_{n_{g y y y}}\right)^{2} & 0 & 0 & 0 & 0 \\
0 & 0 & \left(3 \sigma_{n_{g y y z}}\right)^{2} & 0 & 0 & 0 \\
0 & 0 & 0 & \left(3 \sigma_{n_{a c c x}}\right)^{2} & 0 & 0 \\
0 & 0 & 0 & 0 & \left(3 \sigma_{n_{a c c y}}\right)^{2} & \\
0 & 0 & 0 & 0 & 0 & \left(3 \sigma_{n_{a c c z}}\right)^{2}
\end{array}\right]
$$

The $1 \sigma$ value of the driven noise was calculated by using equation (4.3). After that, process noise covariance matrix was tuned by using $(3 \sigma)^{2}$ value of the driven noise.

$$
Q=\left[\begin{array}{cccccc}
\left(3 \sigma_{n_{\text {gyvw }}}\right)^{2} & 0 & 0 & 0 & 0 & 0 \\
0 & \left(3 \sigma_{n_{\text {gyvw }}}\right)^{2} & 0 & 0 & 0 & 0 \\
0 & 0 & \left(3 \sigma_{n_{\text {gyrw }}}\right)^{2} & 0 & 0 & 0 \\
0 & 0 & 0 & \left(3 \sigma_{n_{\text {accw }}}\right)^{2} & 0 & 0 \\
0 & 0 & 0 & 0 & \left(3 \sigma_{n_{\text {accw }}}\right)^{2} & \\
0 & 0 & 0 & 0 & 0 & \left(3 \sigma_{n_{\text {accw }}}\right)^{2}
\end{array}\right]
$$




\section{CHAPTER 7}

\section{SIMULATION RESULTS}

The main motivation of this chapter is explaining the simulation models and algorithms which were designed and developed within the scope of this thesis study.

\subsection{IMU Error Model Simulation}

IMU error model was built by using Simulink software. Sensor error parameters, gyroscope and accelerometer bias, scale factor, misalignment, random noise error, bias instability and gyroscope g-sensitive bias coefficients were fed into the simulation. These parameters were chosen from actual accelerometer and gyroscope parameters. Deterministic and stochastic sensor error parameters which were used in IMU error model simulation are presented in Table 6 and Table 7.

Purpose of simulation: Observing sensor error effects and generating inputs for deterministic and stochastic error estimation algorithm.

Simulation input: Reference (true) angular rate and acceleration which were used to simulate multi-rate dynamic test and multi-position static test.

Simulation output: Simulated IMU outputs were generated according to the gyroscope and accelerometer error model. The raw IMU measurements were formed by adding errors according to equation (4.5) and (4.7). 
Three different IMU error model simulations were performed within the scope of this thesis study. First of all, the reference state values of multi-position static test and multi-position dynamic test were generated. (States of multi-position static test and multi-rate dynamic test are explained in Chapter 5) After that, multi-position test was simulated in order to create input for deterministic error estimation algorithm.

These simulation outputs were used to determine accelerometer bias, scale factor and misalignment error. Additionally gyroscope bias and g-dependent bias coefficient were estimated from multi-position test simulation output. Similarly, multi-rate dynamic test was simulated. Gyroscope scale factor and misalignment errors were obtained by processing this simulation's outputs.

Finally, IMU error model simulation was used to create a measurement data for Kalman filter algorithm. Outputs of this simulation were corrected by performing IMU error compensation simulation and then corrected outputs fed to the Kalman filter algorithm as measurement.

Table 6 Simulated IMU Deterministic Error Parameters

\begin{tabular}{|c|c|}
\hline $\begin{array}{c}\text { Gyroscope Error } \\
\text { Parameters }\end{array}$ & Value of the parameter \\
\hline x axis scale factor error & $10817 \mathrm{ppm}$ \\
\hline y axis scale factor error & $9124 \mathrm{ppm}$ \\
\hline z axis scale factor error & $15529 \mathrm{ppm}$ \\
\hline x axis bias & $1.1464 \mathrm{deg} / \mathrm{s}$ \\
\hline y axis bias & $1.1241 \mathrm{deg} / \mathrm{s}$ \\
\hline z axis bias & $1.6820 \mathrm{deg} / \mathrm{s}$ \\
\hline $\mathrm{x}$ axis acceleration sensitivity & $0.05 \mathrm{deg} / \mathrm{s} / \mathrm{g}$ \\
\hline y axis acceleration sensitivity & $0.05 \mathrm{deg} / \mathrm{s} / \mathrm{g}$ \\
\hline
\end{tabular}


Table 6 continued

\begin{tabular}{|c|c|}
\hline $\mathrm{z}$ axis acceleration sensitivity & $0.05 \mathrm{deg} / \mathrm{s} / \mathrm{g}$ \\
\hline $\mathrm{x}$ axis to $\mathrm{y}$ axis misalignment & $7.5879 \mathrm{mrad}$ \\
\hline $\mathrm{x}$ axis to $\mathrm{z}$ axis misalignment & $3.3769 \mathrm{mrad}$ \\
\hline $\mathrm{y}$ axis to $\mathrm{x}$ axis misalignment & $5.6617 \mathrm{mrad}$ \\
\hline $\mathrm{y}$ axis to $\mathrm{z}$ axis misalignment & $6.3705 \mathrm{mrad}$ \\
\hline $\mathrm{z}$ axis to $\mathrm{x}$ axis misalignment & $7.5009 \mathrm{mrad}$ \\
\hline $\mathrm{z}$ axis to $\mathrm{y}$ axis misalignment & $3.8084 \mathrm{mrad}$ \\
\hline $\begin{array}{c}\text { Accelerometer Error } \\
\text { Parameters }\end{array}$ & \\
\hline $\mathrm{x}$ axis scale factor error & $3916 \mathrm{ppm}$ \\
\hline $\mathrm{y}$ axis scale factor error & 3284 ppm \\
\hline $\mathrm{z}$ axis scale factor error & $4733 \mathrm{ppm}$ \\
\hline $\mathrm{x}$ axis bias & $349.66 \mathrm{mg}$ \\
\hline $\mathrm{y}$ axis bias & $346.36 \mathrm{mg}$ \\
\hline $\mathrm{z}$ axis bias & $250.18 \mathrm{mg}$ \\
\hline $\mathrm{x}$ axis to $\mathrm{y}$ axis misalignment & $3.9824 \mathrm{mrad}$ \\
\hline $\mathrm{x}$ axis to $\mathrm{z}$ axis misalignment & $0.6601 \mathrm{mrad}$ \\
\hline $\mathrm{y}$ axis to $\mathrm{x}$ axis misalignment & $1.6875 \mathrm{mrad}$ \\
\hline $\mathrm{y}$ axis to $\mathrm{z}$ axis misalignment & $0.8487 \mathrm{mrad}$ \\
\hline $\mathrm{z}$ axis to $\mathrm{x}$ axis misalignment & $2.5776 \mathrm{mrad}$ \\
\hline $\mathrm{z}$ axis to $\mathrm{y}$ axis misalignment & $2.7814 \mathrm{mrad}$ \\
\hline
\end{tabular}


Table 7 Simulated IMU Stochastic Error Parameters

\begin{tabular}{|c|c|}
\hline $\begin{array}{l}\text { Gyroscope Error } \\
\text { Paramaters }\end{array}$ & \\
\hline $\mathrm{x}$ axis time constant & 15 seconds \\
\hline $\mathrm{y}$ axis time constant & 15 seconds \\
\hline $\mathrm{z}$ axis time constant & 15 seconds \\
\hline $\mathrm{x}$ axis bias instability $(1 \sigma)$ & $0.005 \mathrm{deg} / \mathrm{s}$ \\
\hline $\mathrm{y}$ axis bias instability $(1 \sigma)$ & $0.005 \mathrm{deg} / \mathrm{s}$ \\
\hline $\mathrm{z}$ axis bias instability $(1 \sigma)$ & $0.005 \mathrm{deg} / \mathrm{s}$ \\
\hline $\mathrm{x}$ axis noise density $(1 \sigma)$ & $0.0015 \mathrm{deg} / \mathrm{s} / \sqrt{H z}$ \\
\hline $\mathrm{y}$ axis noise density $(1 \sigma)$ & $0.0015 \mathrm{deg} / \mathrm{s} / \sqrt{H z}$ \\
\hline $\mathrm{z}$ axis noise density $(1 \sigma)$ & $0.0015 \mathrm{deg} / \mathrm{s} / \sqrt{H z}$ \\
\hline $\begin{array}{c}\text { Accelerometer Error } \\
\text { Parameters }\end{array}$ & \\
\hline $\mathrm{x}$ axis time constant & 1 second \\
\hline $\mathrm{y}$ axis time constant & 1 second \\
\hline $\mathrm{z}$ axis time constant & 1 second \\
\hline $\mathrm{x}$ axis bias instability $(1 \sigma)$ & $0.5 \mathrm{mg}$ \\
\hline $\mathrm{y}$ axis bias instability $(1 \sigma)$ & $0.5 \mathrm{mg}$ \\
\hline $\mathrm{z}$ axis bias instability $(1 \sigma)$ & $0.5 \mathrm{mg}$ \\
\hline $\mathrm{x}$ axis noise density $(1 \sigma)$ & $0.00027 \mathrm{~g} / \sqrt{H z}$ \\
\hline $\mathrm{y}$ axis noise density $(1 \sigma)$ & $0.00027 \mathrm{~g} / \sqrt{\mathrm{Hz}}$ \\
\hline $\mathrm{z}$ axis noise density $(1 \sigma)$ & $0.00027 \mathrm{~g} / \sqrt{\mathrm{Hz}}$ \\
\hline
\end{tabular}

Multi-rate dynamic test and multi-position static test reference inputs are represented with IMU error model simulation outputs in the following figures. 


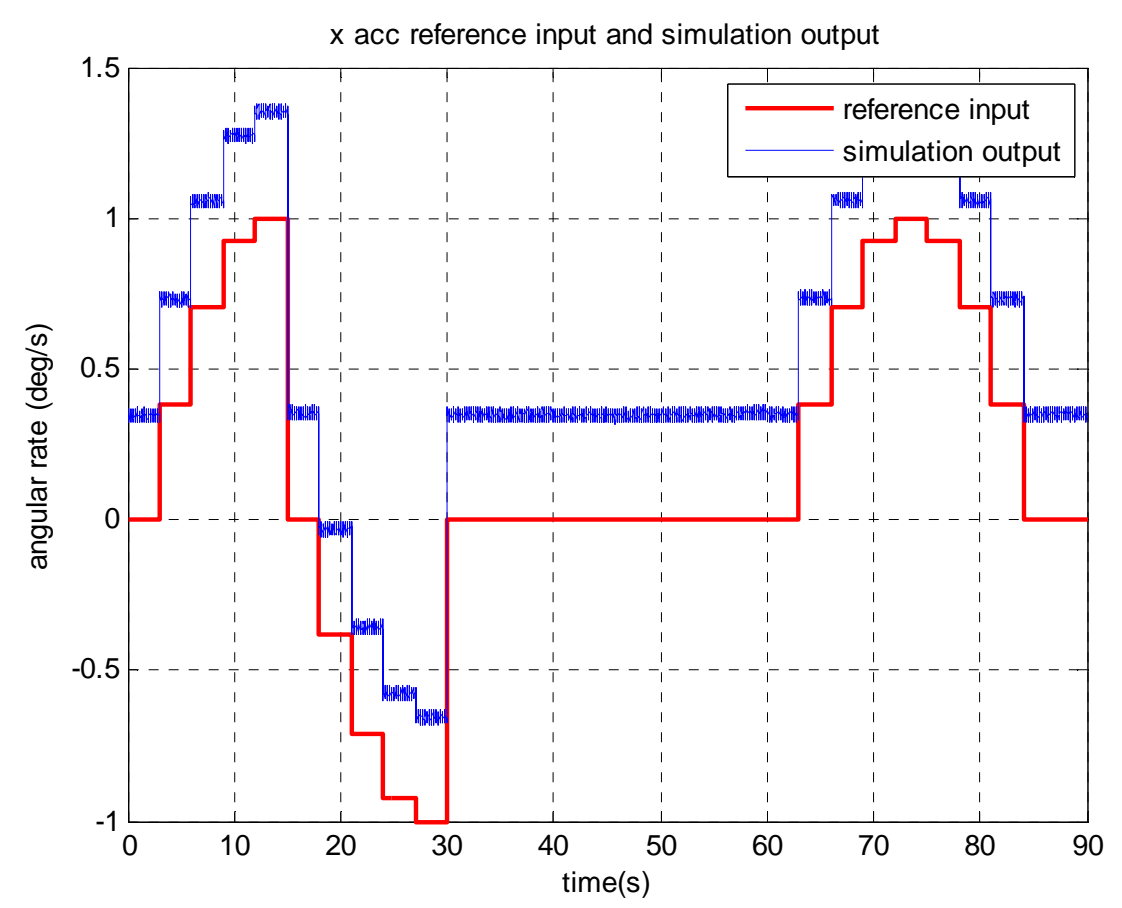

Figure $36 x$ acc reference input and simulation output

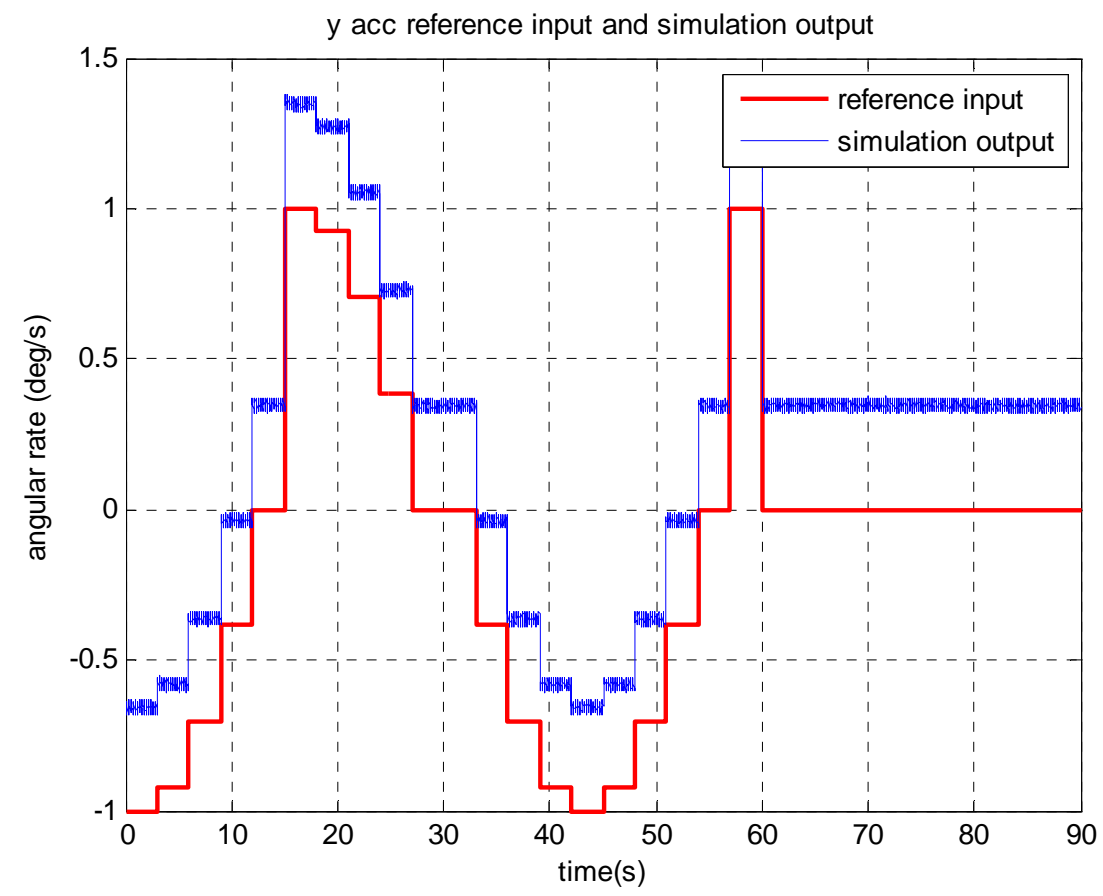

Figure 37 y acc reference input and simulation output 


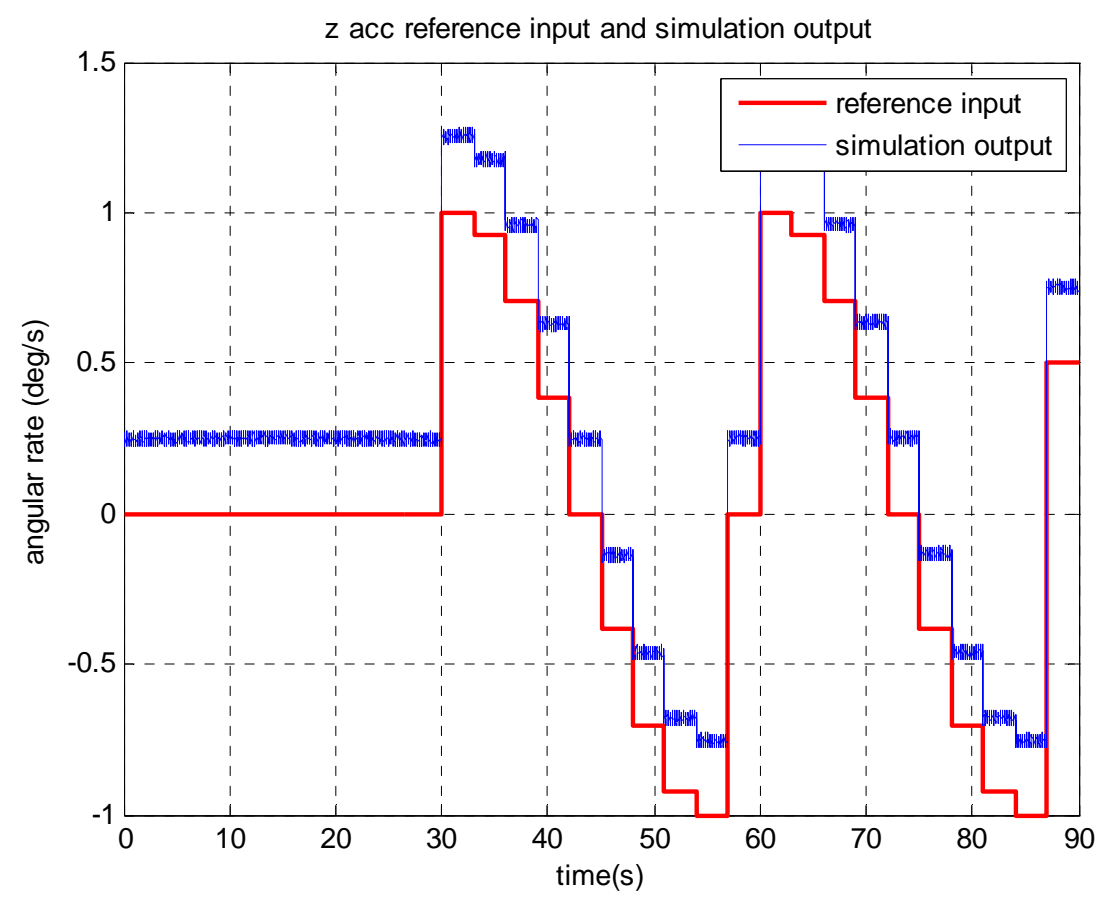

Figure 38 z acc reference input and simulation output

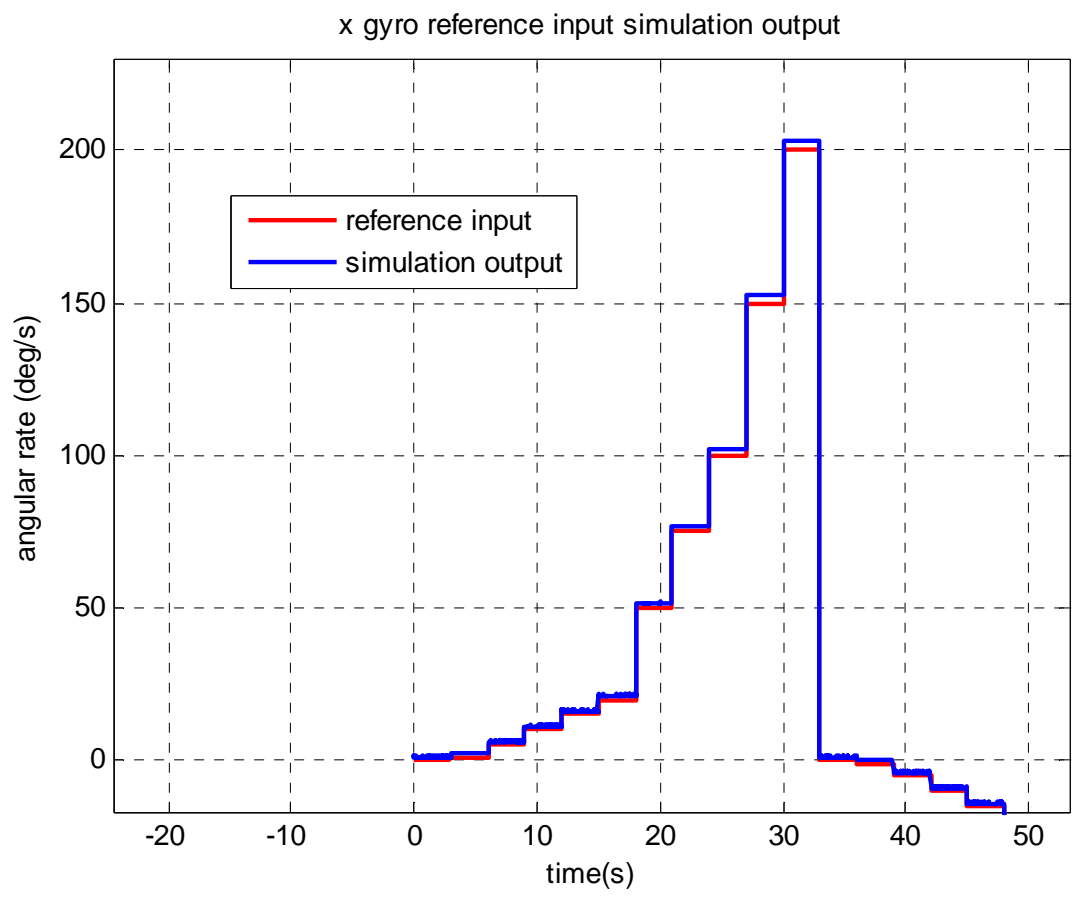

Figure $39 x$ gyro reference input and simulation output 


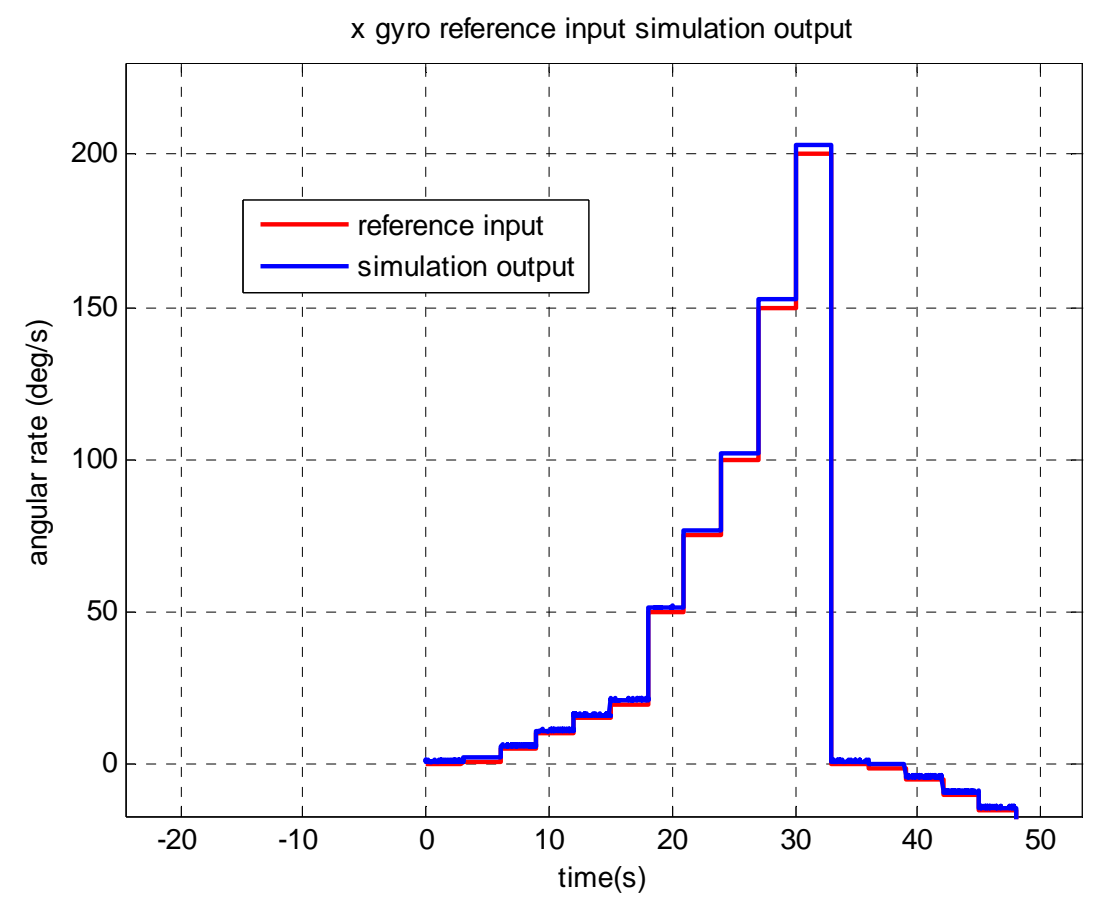

Figure 40 y gyro reference input and simulation output

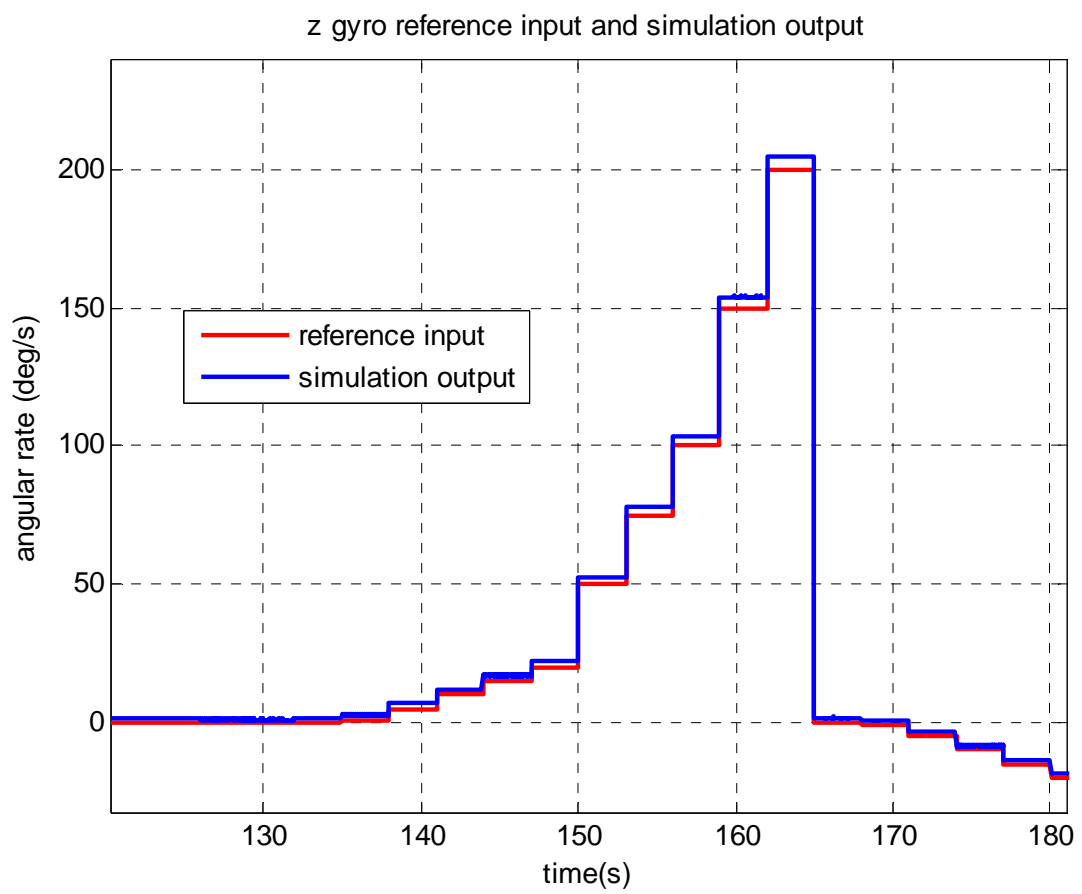

Figure 41 z gyro reference input and simulation output 


\subsection{Deterministic Error Estimation Algorithm}

Deterministic error estimation algorithm was developed by using MATLAB software. Detailed information about data processing steps and algorithm are given in Chapter 5.

Purpose of algorithm: Determination of deterministic IMU error parameters.

Algorithm input: Raw calibration test data. IMU error model simulation outputs (multi-position and multi-rate test simulation) were used as an input for deterministic error estimation algorithm. Furthermore, collected real IMU calibration data can be used as input for the algorithm.

Algorithm output: Estimated accelerometer and gyroscope deterministic error parameters. Comparative table of real and estimated error parameters are presented in Table 8 and Table 9 .

Table 8 Deterministic Error Estimation Algorithm Outputs (Gyroscope)

\begin{tabular}{|l|c|c|c|c|c|c|}
\hline Gyro & $\begin{array}{c}\mathbf{x} \\
\text { real }\end{array}$ & $\begin{array}{c}\mathbf{x} \\
\text { estimated }\end{array}$ & $\begin{array}{c}\mathbf{y} \\
\text { real }\end{array}$ & $\begin{array}{c}\mathbf{y} \\
\text { estimated }\end{array}$ & $\begin{array}{c}\mathbf{z} \\
\text { real }\end{array}$ & $\begin{array}{c}\mathbf{z} \\
\text { estimated }\end{array}$ \\
\hline Bias & 1.1464 & 1.1457 & 1.1241 & 1.1210 & 1.6820 & 1.6756 \\
& $\mathrm{deg} / \mathrm{s}$ & $\mathrm{deg} / \mathrm{s}$ & $\mathrm{deg} / \mathrm{s}$ & $\mathrm{deg} / \mathrm{s}$ & $\mathrm{deg} / \mathrm{s}$ & $\mathrm{deg} / \mathrm{s}$ \\
\hline G-dep bias & 0.05 & 0.0522 & 0.05 & 0.0488 & 0.05 & 0.0526 \\
Coefficient & $\mathrm{deg} / \mathrm{s} / \mathrm{g}$ & $\mathrm{deg} / \mathrm{s} / \mathrm{g}$ & $\mathrm{deg} / \mathrm{s} / \mathrm{g}$ & $\mathrm{deg} / \mathrm{s} / \mathrm{g}$ & $\mathrm{deg} / \mathrm{s} / \mathrm{g}$ & $\mathrm{deg} / \mathrm{s} / \mathrm{g}$ \\
\hline Scale Factor & 10817 & 10802 & 9124 & 9079 & 15529 & 15584 \\
& $\mathrm{ppm}$ & $\mathrm{ppm}$ & $\mathrm{ppm}$ & $\mathrm{ppm}$ & $\mathrm{ppm}$ & $\mathrm{ppm}$ \\
\hline Mrror & $\mathrm{xy}$ & $\mathrm{xy}$ & $\mathrm{yx}$ & $\mathrm{yx}$ & $\mathrm{zx}$ & $\mathrm{zx}$ \\
& 7.5879 & 7.5812 & 5.6617 & 5.6634 & 7.5009 & 7.5156 \\
& $\mathrm{mrad}$ & $\mathrm{mrad}$ & $\mathrm{mrad}$ & $\mathrm{mrad}$ & $\mathrm{mrad}$ & $\mathrm{mrad}$ \\
& $\mathrm{xz}$ & $\mathrm{xz}$ & $\mathrm{yz}$ & $\mathrm{yz}$ & $\mathrm{zy}$ & $\mathrm{zy}$ \\
& 3.3769 & 3.3749 & 6.3705 & 6.3827 & 3.8084 & 3.7935 \\
& $\mathrm{mrad}$ & $\mathrm{mrad}$ & $\mathrm{mrad}$ & $\mathrm{mrad}$ & $\mathrm{mrad}$ & $\mathrm{mrad}$ \\
\hline
\end{tabular}


Table 9 Deterministic Error Estimation Algorithm Outputs (Accelerometer)

\begin{tabular}{|l|c|c|c|c|c|c|}
\hline Acc & $\mathbf{x}$ real & $\begin{array}{c}\mathbf{x} \\
\text { estimated }\end{array}$ & $\mathbf{y}$ real & $\begin{array}{c}\mathbf{y} \\
\text { estimated }\end{array}$ & $\mathbf{z}$ real & $\begin{array}{c}\mathbf{z} \\
\text { estimated }\end{array}$ \\
\hline Bias & 349.66 & 349.6557 & 346.36 & 346.2070 & 250.18 & 249.9346 \\
& $\mathrm{mg}$ & $\mathrm{mg}$ & $\mathrm{mg}$ & $\mathrm{mg}$ & $\mathrm{mg}$ & $\mathrm{mg}$ \\
\hline Scale Factor & 3916 & 4003 & 3284 & 3106 & 4733 & 4964 \\
Error & $\mathrm{ppm}$ & $\mathrm{ppm}$ & $\mathrm{ppm}$ & $\mathrm{ppm}$ & $\mathrm{ppm}$ & $\mathrm{ppm}$ \\
\hline Misalignment & $\mathrm{xy}$ & $\mathrm{xy} 3.8995$ & $\mathrm{yx}$ & $\mathrm{yx}$ & $\mathrm{zx}$ & $\mathrm{zx} 2.5641$ \\
& 3.9824 & $\mathrm{mrad}$ & 1.6875 & 1.4454 & 2.5776 & $\mathrm{mrad}$ \\
& $\mathrm{mrad}$ & $\mathrm{xz} 0.5115$ & $\mathrm{mrad}$ & $\mathrm{mrad}$ & $\mathrm{mrad}$ & $\mathrm{zy} 2.7139$ \\
& $\mathrm{xz}$ & $\mathrm{mrad}$ & $\mathrm{yz}$ & $\mathrm{yz}$ & $\mathrm{zy}$ & $\mathrm{mrad}$ \\
& 0.6601 & & 0.8487 & 0.9496 & 2.7814 & \\
& $\mathrm{mrad}$ & & $\mathrm{mrad}$ & $\mathrm{mrad}$ & $\mathrm{mrad}$ & \\
\hline
\end{tabular}

The difference between the actual error parameters and the estimated parameters comes from the effect of the sensor noise and the stochastic errors such as bias instability.

In addition to the multi-position static and multi-rate dynamic test method, six position direct method was performed to estimate deterministic error parameters. Results of the six-position direct method were compared with the multi-position and multi-rate method. IMU error model simulation was run with six-position direct method reference input (Reference angular rate is equal to $10 \mathrm{deg} / \mathrm{s}$ and reference acceleration is equal to gravity.). Simulation outputs are given in the following figures. Deterministic errors of simulated IMU were estimated by using equations (5.1), (5.2), (5.3) and (5.4). IMU error model simulation outputs of six-position direct method are presented with reference inputs of six-position direct method in Figure 42 to Figure 47. 


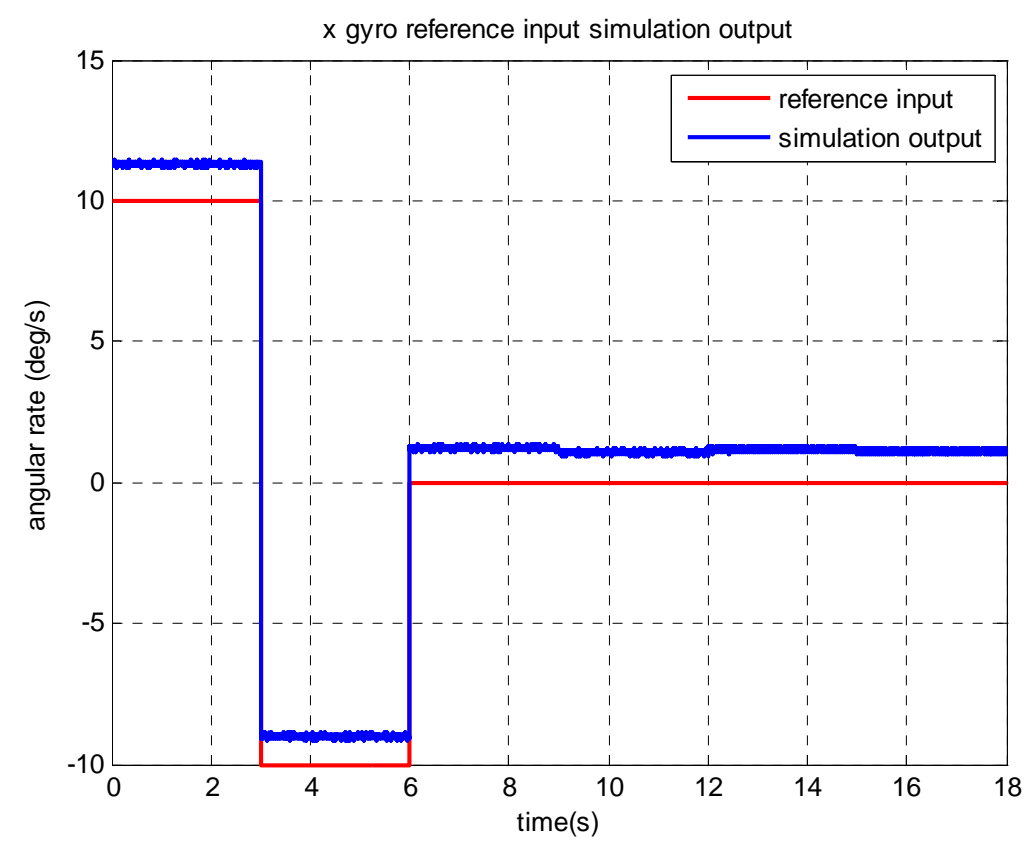

Figure $42 x$ gyro reference input and simulation output (six position test)

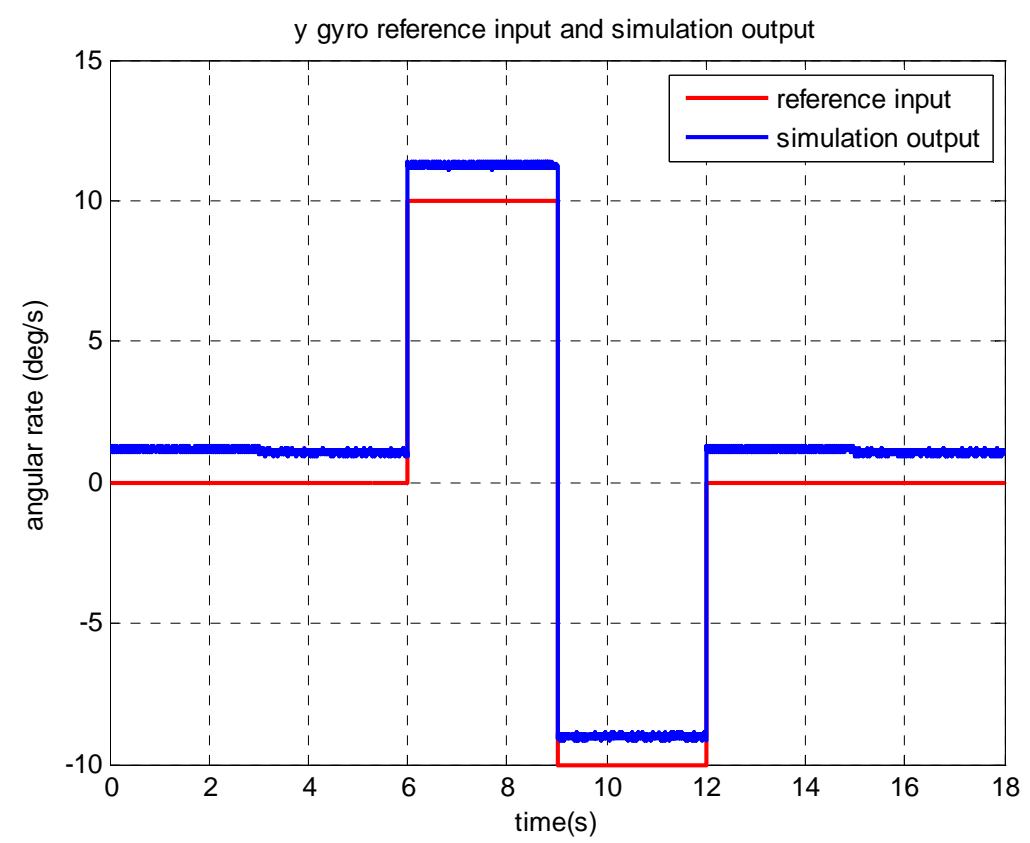

Figure 43 y gyro reference input and simulation output (six position test) 


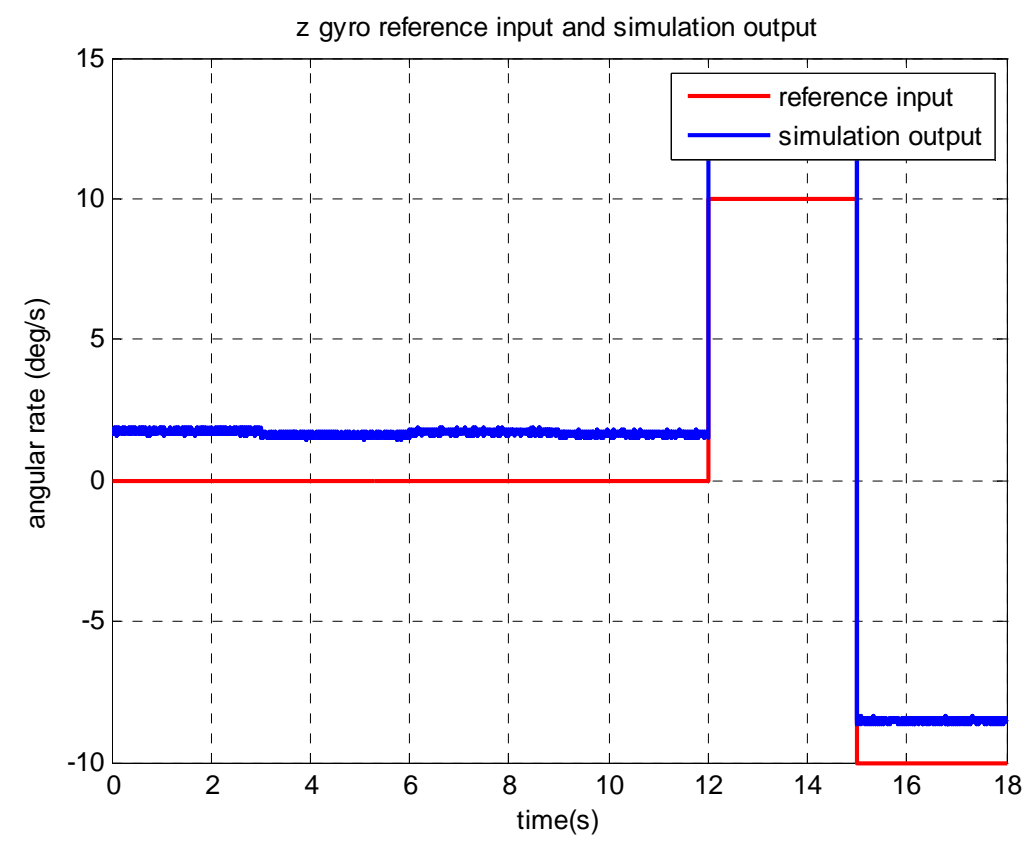

Figure 44 z gyro reference input and simulation output (six position test)

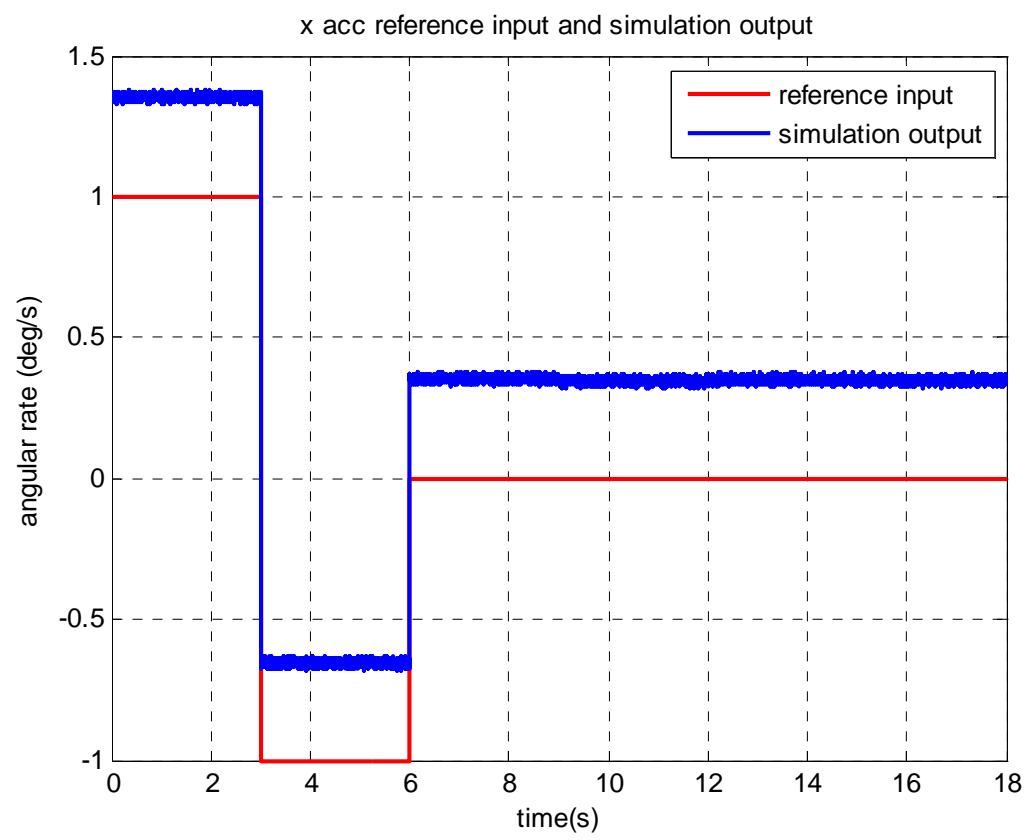

Figure $45 x$ acc reference input and simulation output (six position test) 


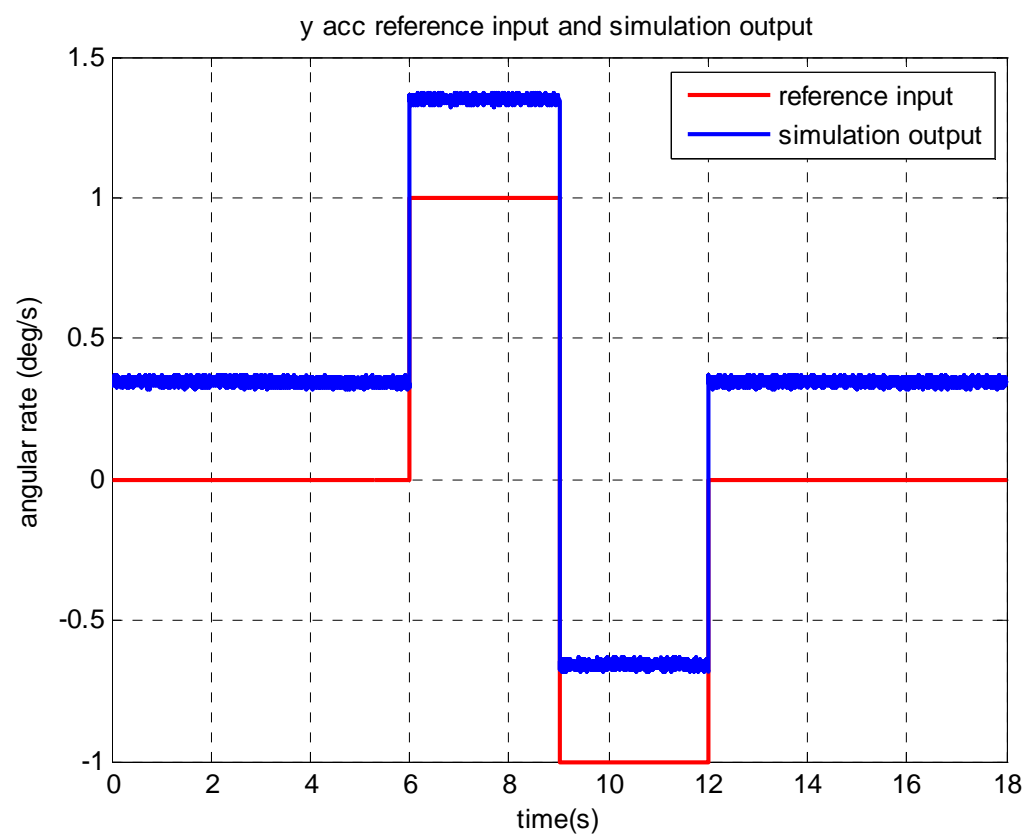

Figure 46 y acc reference input and simulation output (six position test)

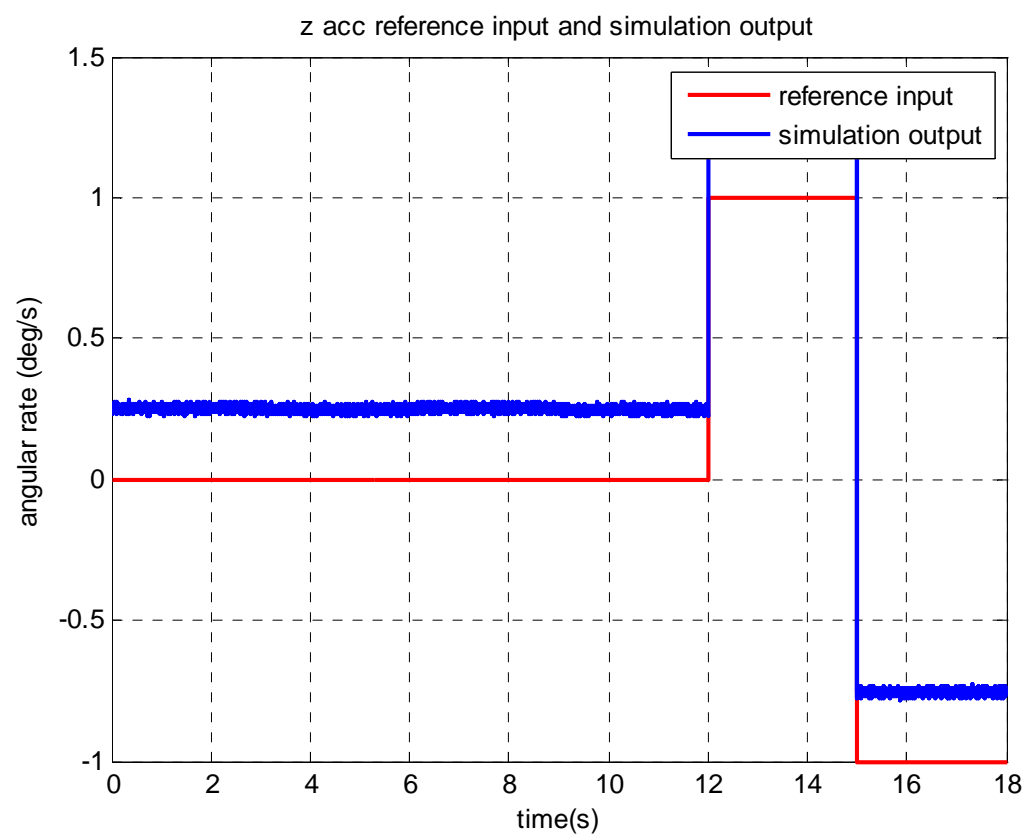

Figure 47 z acc reference input and simulation output (six position test) 
Table 10 Six position direct method results (gyroscopes)

\begin{tabular}{|l|c|c|c|c|c|c|}
\hline Gyro & $\mathbf{x}$ real & $\begin{array}{c}\mathbf{x} \\
\text { estimated }\end{array}$ & $\mathbf{y}$ real & $\begin{array}{c}\mathbf{y} \\
\text { estimated }\end{array}$ & $\mathbf{z}$ real & $\begin{array}{c}\mathbf{z} \\
\text { estimated }\end{array}$ \\
\hline Bias & 1.1464 & 1.1449 & 1.1241 & 1.1248 & 1.6820 & 1.6832 \\
& $\mathrm{deg} / \mathrm{s}$ & $\mathrm{deg} / \mathrm{s}$ & $\mathrm{deg} / \mathrm{s}$ & $\mathrm{deg} / \mathrm{s}$ & $\mathrm{deg} / \mathrm{s}$ & $\mathrm{deg} / \mathrm{s}$ \\
\hline Scale & 10817 & $10980 \mathrm{ppm}$ & 9124 & 9288 & 15529 & 15690 \\
Factor & $\mathrm{ppm}$ & & $\mathrm{ppm}$ & $\mathrm{ppm}$ & $\mathrm{ppm}$ & $\mathrm{ppm}$ \\
Error & & & & & & \\
\hline
\end{tabular}

Table 11 Six position direct method results (accelerometers)

\begin{tabular}{|l|c|c|c|c|c|c|}
\hline Acc & $\mathbf{x}$ real & $\begin{array}{c}\mathbf{x} \\
\text { estimated }\end{array}$ & $\mathbf{y}$ real & $\begin{array}{c}\mathbf{y} \\
\text { estimated }\end{array}$ & $\mathbf{z}$ real & $\begin{array}{c}\mathbf{z} \\
\text { estimated }\end{array}$ \\
\hline Bias & $\begin{array}{c}349.66 \\
\mathrm{mg}\end{array}$ & $\begin{array}{c}349.401 \\
\mathrm{mg}\end{array}$ & $\begin{array}{c}346.36 \\
\mathrm{mg}\end{array}$ & $\begin{array}{c}346.496 \\
\mathrm{mg}\end{array}$ & $\begin{array}{c}250.18 \\
\mathrm{mg}\end{array}$ & $\begin{array}{c}250.445 \\
\mathrm{mg}\end{array}$ \\
\hline Scale & 3916 & $3581 \mathrm{ppm}$ & $\begin{array}{c}3284 \\
\mathrm{ppm}\end{array}$ & $\begin{array}{c}2950 \\
\mathrm{ppm}\end{array}$ & $\begin{array}{c}4733 \\
\mathrm{ppm}\end{array}$ & $\begin{array}{c}4399 \\
\mathrm{ppm}\end{array}$ \\
Factor & $\mathrm{ppm}$ & & & & & \\
\hline
\end{tabular}

It can be observed from Table 10 and Table 11, performance of six-position direct method is lower than multi-position and multi-rate test methods. The estimated parameters which are determined by using multi-position and multi-rate test data are closer to actual error parameters. Also, misalignment error could not be estimated by six-position direct method. As a result, multi-position and multi-rate test method are more useful than six-position direct method.

\subsection{IMU Error Compensation (Calibration) Model Simulation}

IMU error compensation model was built by using Simulink software. Equation (5.17) and (5.18) were implemented to simulate the error compensation algorithm. Estimated deterministic error parameters was inserted into simulation blocks as calibration parameters. 
Simulation input: Raw IMU measurements. IMU error model simulation results or collected IMU data can be used as an input for IMU error compensation simulation.

Simulation output: Error compensated angular rate and linear acceleration measurements. Deterministic error effects were eliminated from raw IMU measurements but stochastic error effects could not be compensated.

Purpose of simulation: Compensation of deterministic error parameters of an IMU. The optimum bias, scale factor and misalignment were estimated according to calibration test states. As shown in Table- 8 and Table-9 estimated error parameters are not equal to actual error parameters. The difference between estimated and actual error parameters is caused by the effect of sensor bias instability and random sensor noise.

The raw data was calibrated with IMU error compensation model by using the estimated deterministic error parameters. After the calibration process, the difference between the calibrated data and the reference data (IMU error model input data) was calculated to evaluate the performance of the error compensation algorithm. This difference represents the IMU's total residual error. The magnitude of the residual errors vary depending on the difference between the actual and the estimated error parameters. Furthermore, the magnitude of bias instability and random sensor noise also affect the magnitude of residual errors.

The following figures show the changes in IMU's total residual errors according to the reference inputs. It was observed that the magnitude of total residual error increases when the magnitude of the input rate and acceleration increases. This situation can be explained with the observability of the scale factor error and misalignment error. The effect of residual scale factor error and misalignment error becomes observable at high rates and accelerations. Besides, the effect of residual bias can be observable at all rates and accelerations. As a result total residual errors include the effects of residual scale factor, bias and misalignment errors. 


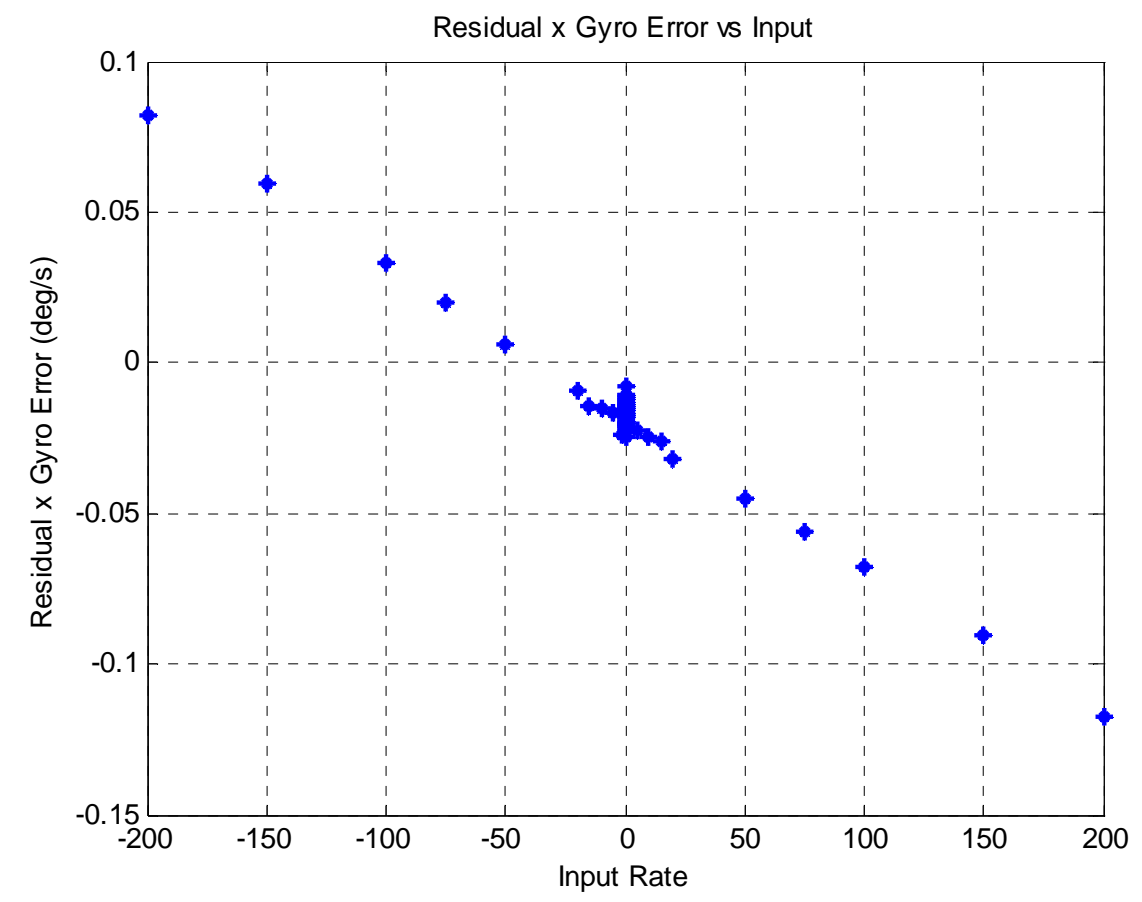

Figure $48 x$ gyro residual error distribution

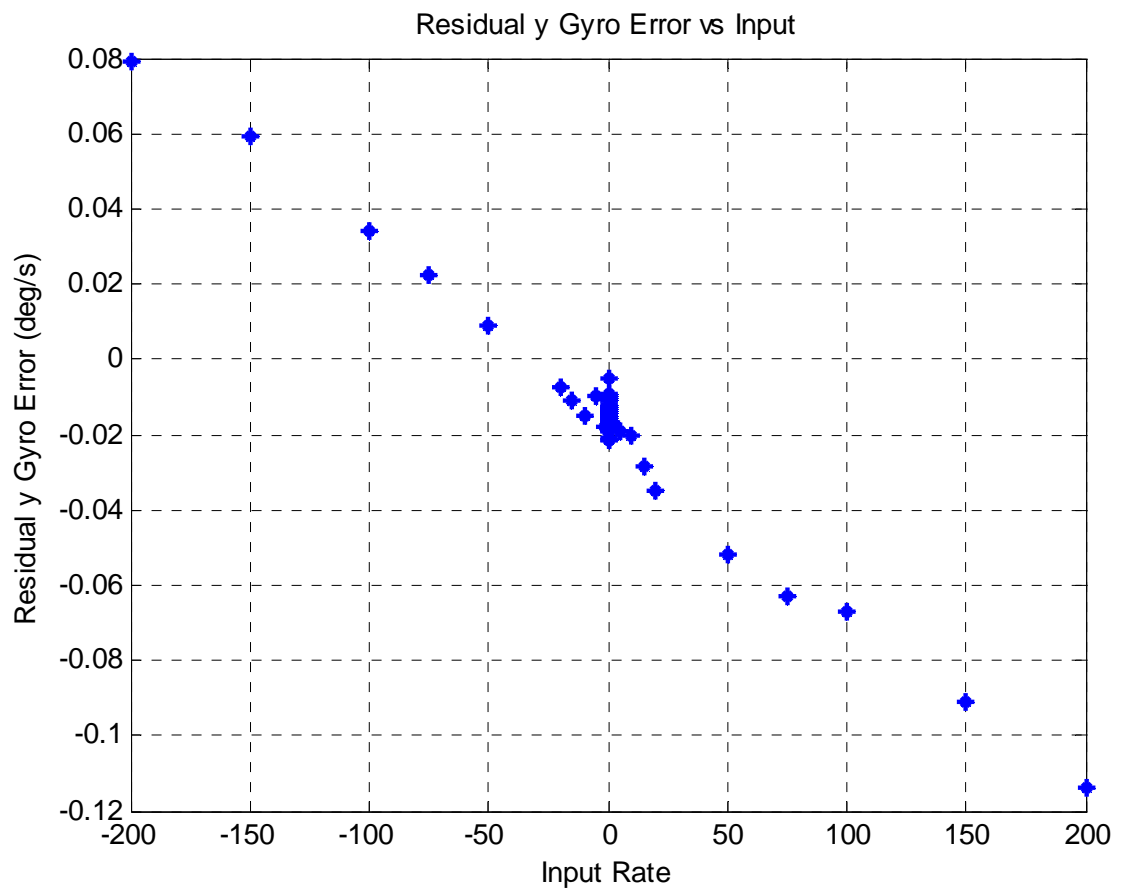

Figure 49 y gyro residual error distribution 


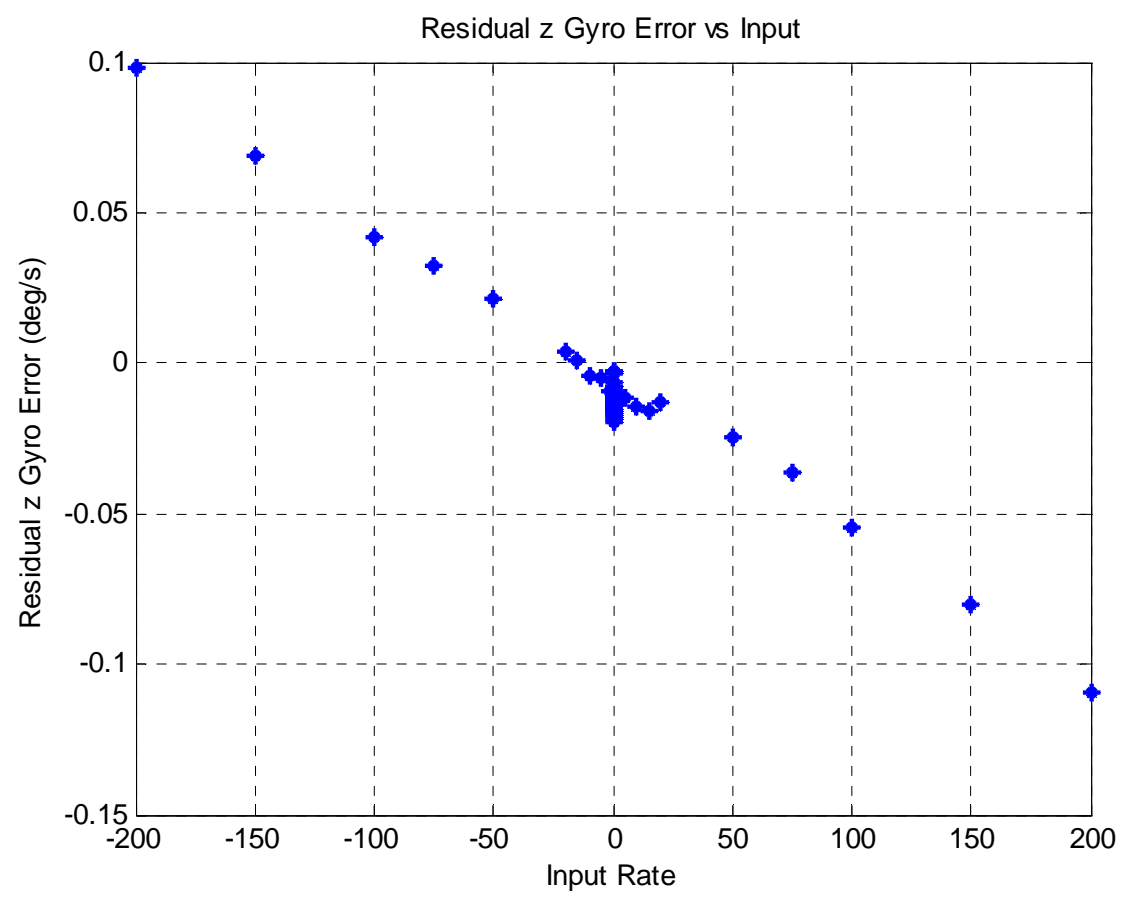

Figure 50 z gyro residual error distribution

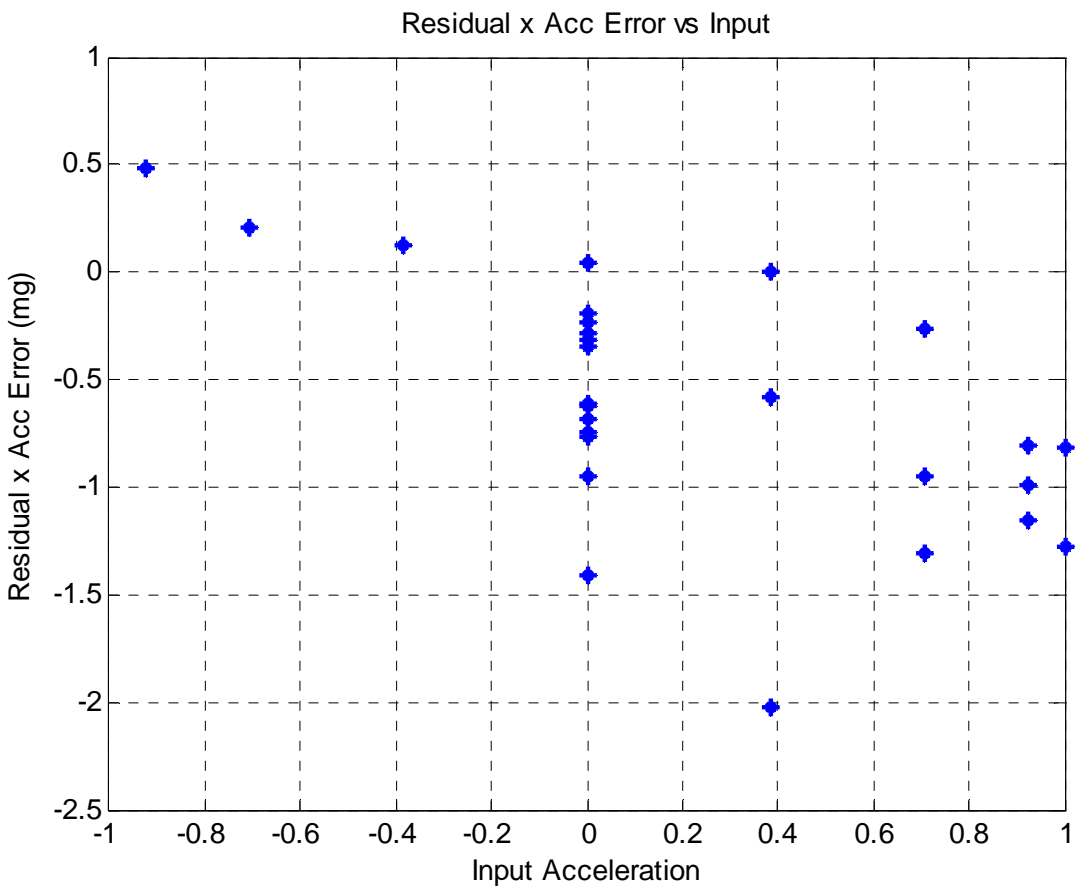

Figure $51 x$ acc residual error distribution 


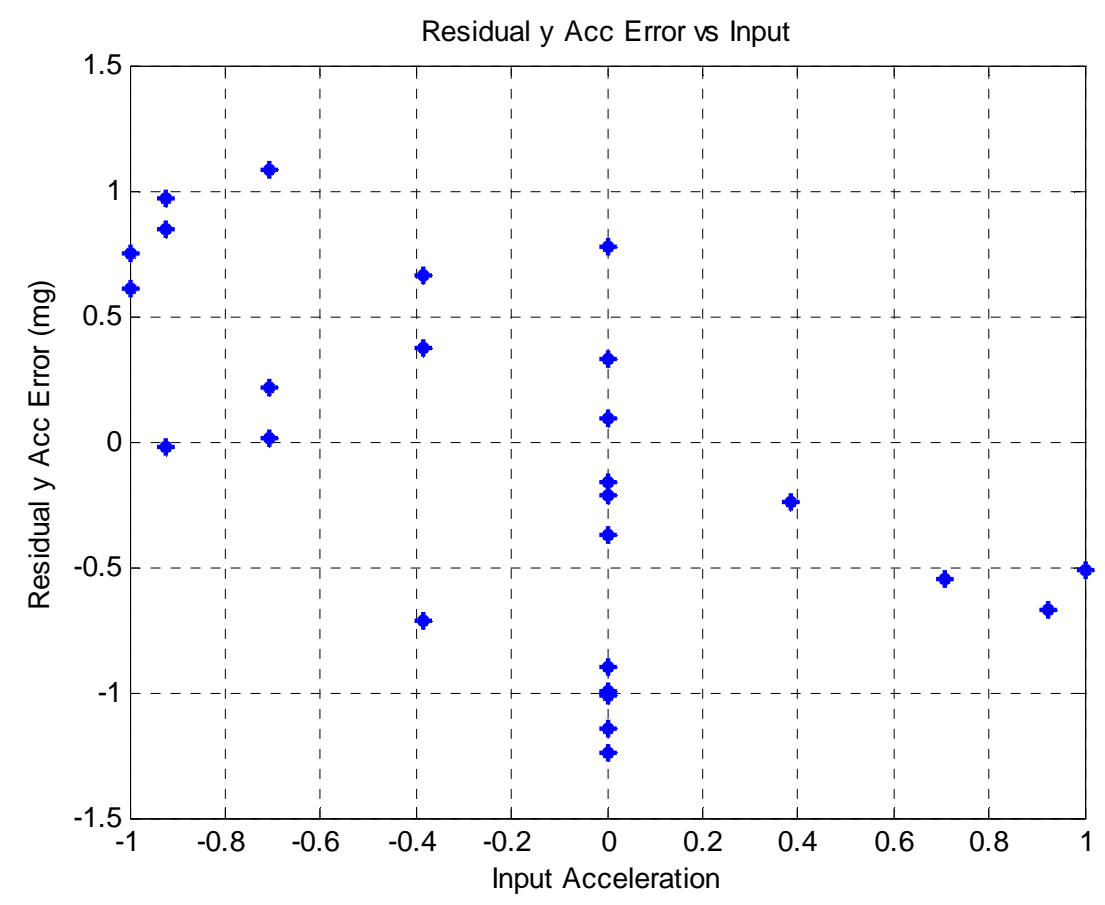

Figure 52 y acc residual error distribution

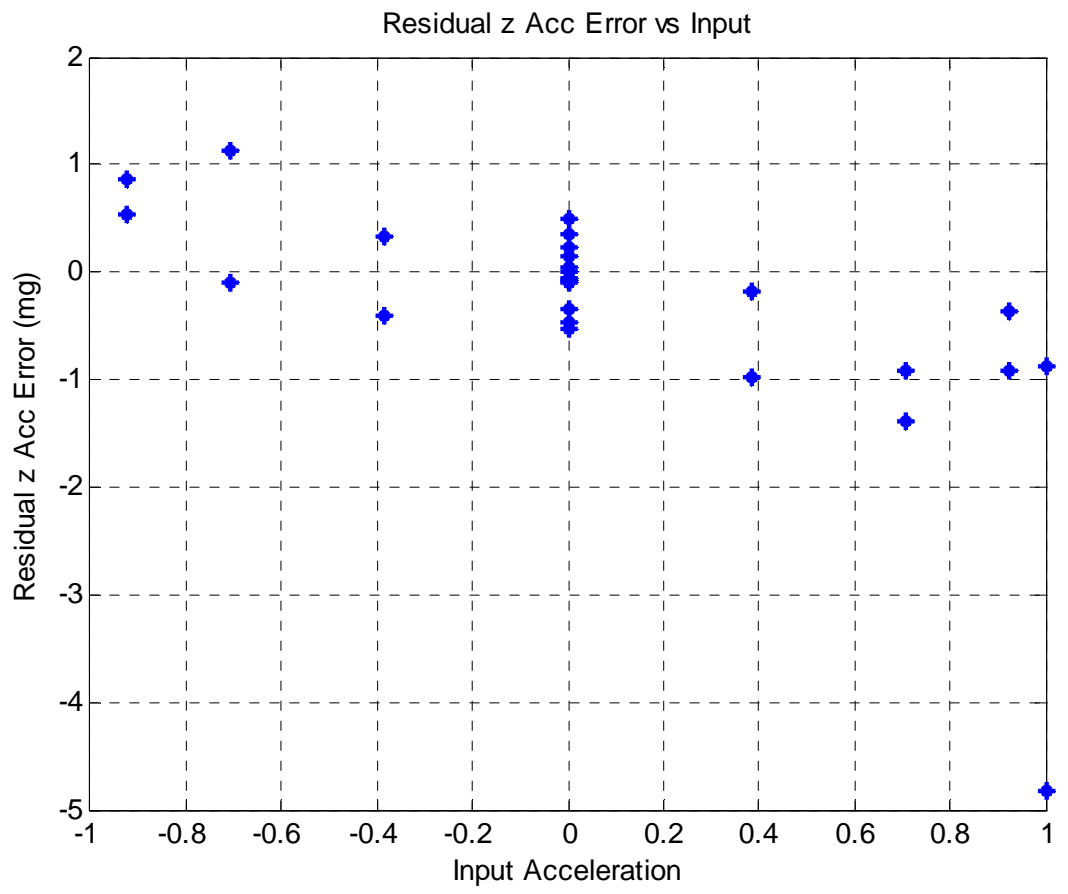

Figure 53 z acc residual error distribution 
The RMS value and the repeatability of the total residual error determine the performance limitations of an IMU. The simulated IMU's residual error RMS value and repeatability are given in Table-12

Table 12 Simulated IMU's residual error

\begin{tabular}{|c|c|c|c|}
\hline & $\mathrm{X}$ & $\mathrm{Y}$ & $\mathrm{Z}$ \\
\hline $\begin{array}{c}\text { Gyro Error RMS } \\
\text { value }\end{array}$ & $109,8451 \mathrm{deg} / \mathrm{h}$ & $107,1827 \mathrm{deg} / \mathrm{h}$ & $98,1508 \mathrm{deg} / \mathrm{h}$ \\
\hline $\begin{array}{c}\text { Acc Error RMS } \\
\text { value }\end{array}$ & $0,8190 \mathrm{mg}$ & $0,6837 \mathrm{mg}$ & $1,0323 \mathrm{mg}$ \\
\hline $\begin{array}{c}\text { Gyro Error } \\
\text { Repeatability }\end{array}$ & $95.6266 \mathrm{deg} / \mathrm{h}$ & $95.8356 \mathrm{deg} / \mathrm{h}$ & $96.3739 \mathrm{deg} / \mathrm{h}$ \\
\hline $\begin{array}{c}\text { Acc Error } \\
\text { Repeatability }\end{array}$ & $0,6080 \mathrm{mg}$ & $0,7057 \mathrm{mg}$ & $1,1307 \mathrm{mg}$ \\
\hline
\end{tabular}

The standard deviation, $1 \sigma$, of the residual errors is called as the error repeatability. The error distribution is assumed to be Gaussian. Therefore, the $3 \sigma$ value of the error repeatability indicates the maximum error limit of an IMU. [25,26]

\subsection{Stochastic Error Estimation Algorithm}

Stochastic error estimation algorithm was built by using MATLAB software. Equations (3.5), (3.6), (3.7), (3.8) and (3.9) were implemented to process the Kalman filter algorithm. Bias instabilities of gyroscopes and accelerometers estimations were observed from the outputs of this algorithm. In order to observe the effects of parameter tuning and sensor properties, the algorithm was processed under different conditions.

Algorithm input: The difference between the calibrated IMU data and the reference input.

Algorithm output: Instantaneous values of accelerometer and gyroscope bias instabilities. 
Purpose of algorithm: Estimation of stochastic error parameters of an IMU. Stochastic properties of the simulated IMU are given in Table 13. These parameters were generated accordance with real MEMS gyroscope and MEMS accelerometer specifications. Furthermore, properties of the algorithm are also given in Table 14.

Table 13 Stochastic Properties of the IMU Sensors

\begin{tabular}{|l|c|c|c|}
\hline & $\begin{array}{c}\text { Correlation Time } \\
\text { (Time Constant) }\end{array}$ & $\begin{array}{l}\text { Bias Instability } \\
(1 \sigma)\end{array}$ & $\begin{array}{l}\text { Sensor Noise } \\
\text { Density }(1 \sigma)\end{array}$ \\
\hline Gyroscopes & 15 seconds & $0.005 \mathrm{deg} / \mathrm{s}$ & $\begin{array}{c}0.0015 \\
\mathrm{deg} / \mathrm{s} / \sqrt{\mathrm{Hz}}\end{array}$ \\
\hline Accelerometers & 1 second & $0.5 \mathrm{mg}$ & 0.00027 \\
$\mathrm{~g} / \sqrt{\mathrm{Hz}}$
\end{tabular}

Table 14 Properties of the simulation

\begin{tabular}{|c|c|}
\hline Simulation Properties & \\
\hline Sampling interval $(\mathrm{dt})$ & 0.001 second \\
\hline Processing duration & 100000 iteration $=100$ seconds \\
\hline
\end{tabular}

State transition matrix, A, was calculated according to the stochastic properties of the simulated IMU and stochastic model of sensor bias instability. (Detailed information about $\mathrm{A}$ is given in Chapter 6. )

$$
A=\left[\begin{array}{cccccc}
0.9999 & 0 & 0 & 0 & 0 & 0 \\
0 & 0.9999 & 0 & 0 & 0 & 0 \\
0 & 0 & 0.9999 & 0 & 0 & 0 \\
0 & 0 & 0 & 0.9999 & 0 & 0 \\
0 & 0 & 0 & 0 & 0.9999 & 0 \\
0 & 0 & 0 & 0 & 0 & 0.9999
\end{array}\right]
$$

Initial value of error covariance matrix, $P_{0}$, was calculated accordance with equation (6.5). 


$$
P_{0}=\left[\begin{array}{ccccccc}
(0.0150)^{2} & 0 & 0 & 0 & 0 & 0 \\
0 & (0.0150)^{2} & 0 & 0 & 0 & 0 \\
0 & 0 & (0.0150)^{2} & 0 & 0 & 0 \\
0 & 0 & 0 & (0.0015)^{2} & 0 & 0 \\
0 & 0 & 0 & 0 & (0.0015)^{2} & 0 \\
0 & 0 & 0 & 0 & 0 & (0.0015)^{2}
\end{array}\right]
$$

Similarly, the measurement noise covariance matrix, $R$, was calculated by using equation (6.6). The calculated numerical value of related matrix is given below.

$$
R=\left[\begin{array}{cccccc}
(0.1422)^{2} & 0 & 0 & 0 & 0 & 0 \\
0 & (0.1422)^{2} & 0 & 0 & 0 & 0 \\
0 & 0 & (0.1422)^{2} & 0 & 0 & 0 \\
0 & 0 & 0 & (0.0255)^{2} & 0 & 0 \\
0 & 0 & 0 & 0 & (0.0255)^{2} & 0 \\
0 & 0 & 0 & 0 & 0 & (0.0255)^{2}
\end{array}\right]
$$

Finally, the process noise covariance matrix, $Q$, was calculated by using equation (6.7). The calculated numerical value of related matrix is given below.

$$
Q=\left[\begin{array}{ccccccc}
\left(2.999 * 10^{-5}\right)^{2} & 0 & 0 & 0 & 0 & 0 \\
0 & \left(2.999 * 10^{-5}\right)^{2} & 0 & 0 & 0 & 0 \\
0 & 0 & \left(2.999 * 10^{-5}\right)^{2} & 0 & 0 & 0 \\
0 & 0 & 0 & \left(6.705 * 10^{-5}\right)^{2} & 0 & 0 \\
0 & 0 & 0 & 0 & \left(6.705 * 10^{-5}\right)^{2} & 0 \\
0 & 0 & 0 & 0 & 0 & \left(6.705 * 10^{-5}\right)^{2}
\end{array}\right]
$$

Results of the algorithm (x,y,z channel gyroscope and accelerometer bias instability estimation) are given in Figure 58 to 63 . Additionally, changes in the Kalman gain and the error covariance values are presented in Figures 54 ,55, 56 and 57. 


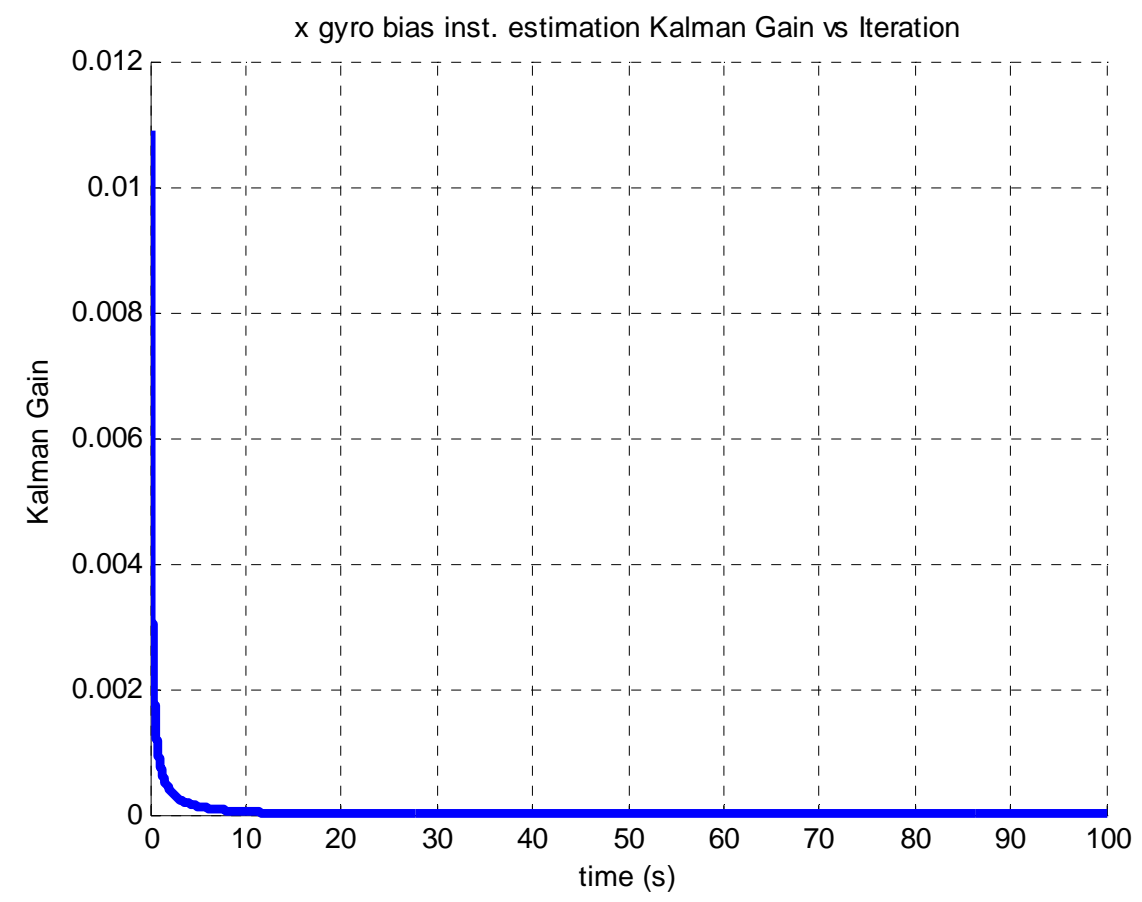

Figure 54 Gyroscope bias instability estimation Kalman gain variation

The Kalman gain variations for the $\mathrm{x}, \mathrm{y}, \mathrm{z}$ gyro bias instability estimations are identical. Therefore, only x gyro's Kalman gain variation is presented.

At the end of 10 seconds, Kalman gain, which was used in gyro bias instability estimation, approached the equilibrium. This situation shows that the estimated gyro bias instability states converged to actual bias instability. On the other hand, convergence of the accelerometer bias instability estimation happened earlier than the gyro bias instability estimation convergence. On the basis of this point, it can be said that convergence accelerometer bias instability estimation occurred before the convergence of gyroscope bias instability estimation. 


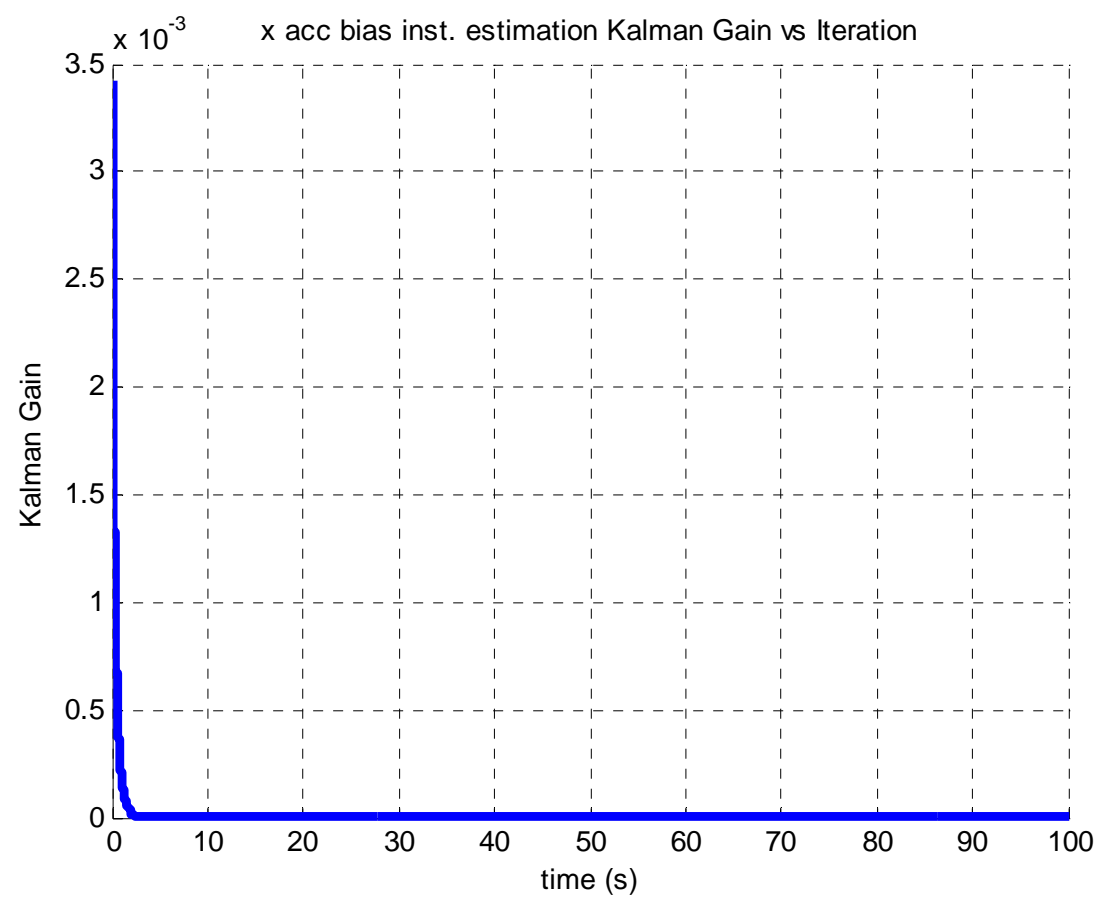

Figure 55 Accelerometer bias instability estimation Kalman gain variation

The Kalman gain variations for the $\mathrm{x}, \mathrm{y}, \mathrm{z}$ accelerometer bias instability estimations are identical. Therefore, only x acceleromreter's Kalman gain variation is presented.

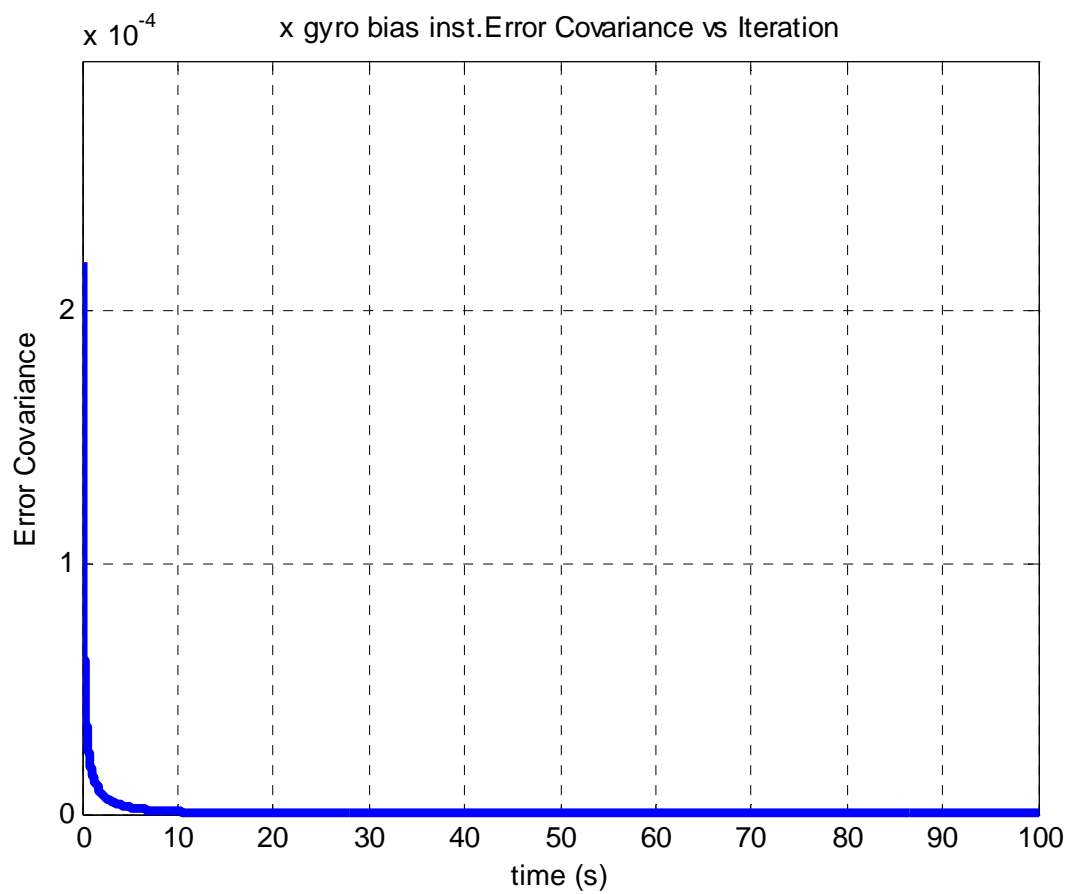

Figure 56 Gyroscope bias instability estimation error covariance variation 
The error covariance variations for the $\mathrm{x}, \mathrm{y}, \mathrm{z}$ gyro bias instability estimations are identical. Therefore, only x gyro's error covariance variation is presented.

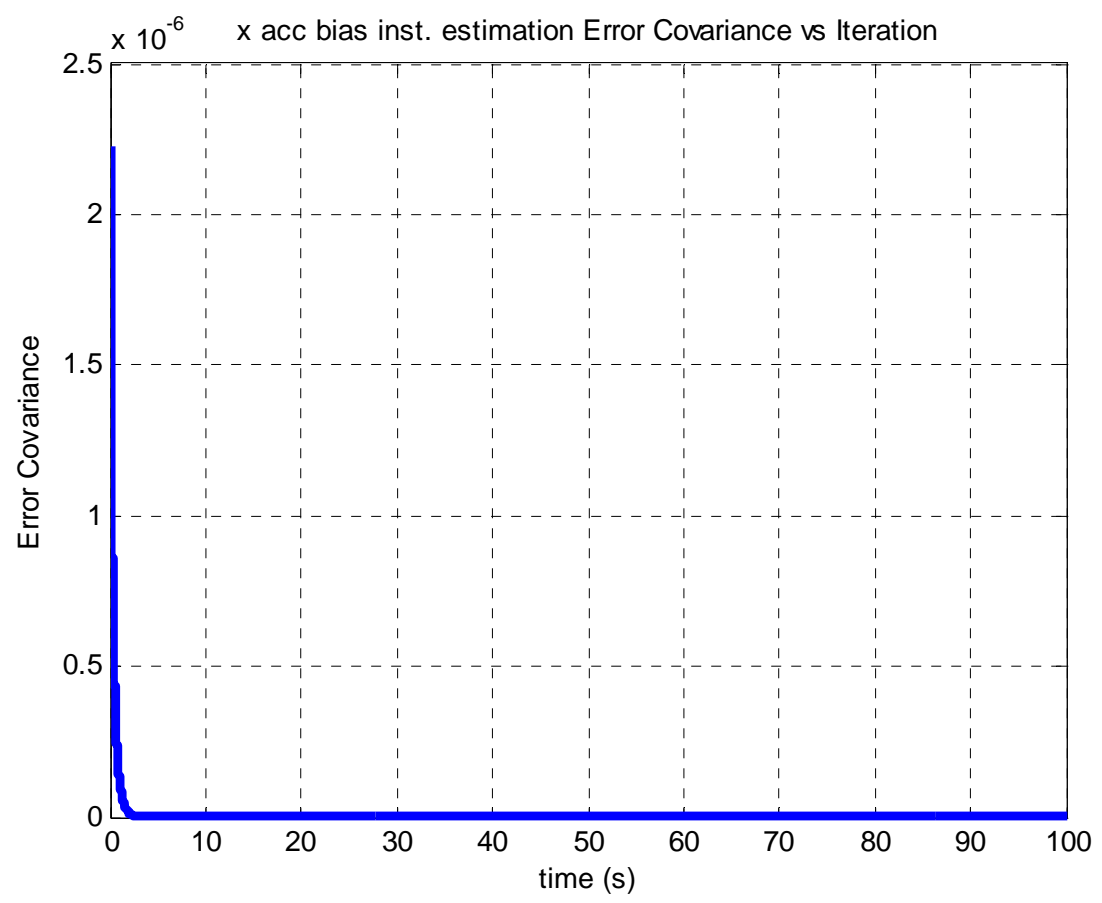

Figure 57 Accelerometer bias instability estimation error covariance variation

The error covariance variations for the $\mathrm{x}, \mathrm{y}, \mathrm{z}$ accelerometer bias instability estimations are identical. Therefore, only x accelerometer's variation is presented.

Similar to the Kalman gain convergence, accelerometer bias instability estimation error covariance approached equilibrium before the bias instability estimation error covariance. It can be also explained by more rapid convergence of accelerometer bias instability estimation. 


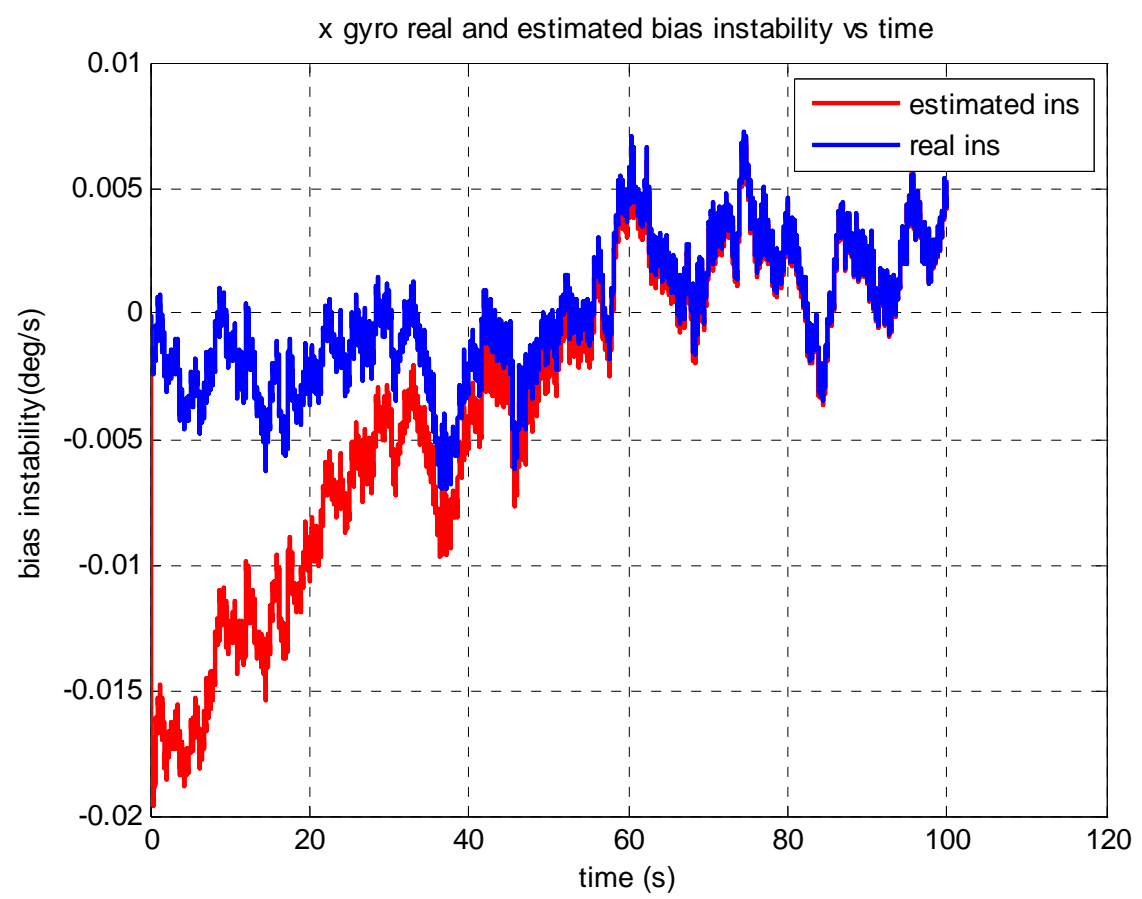

Figure 58 x gyro estimated bias instability

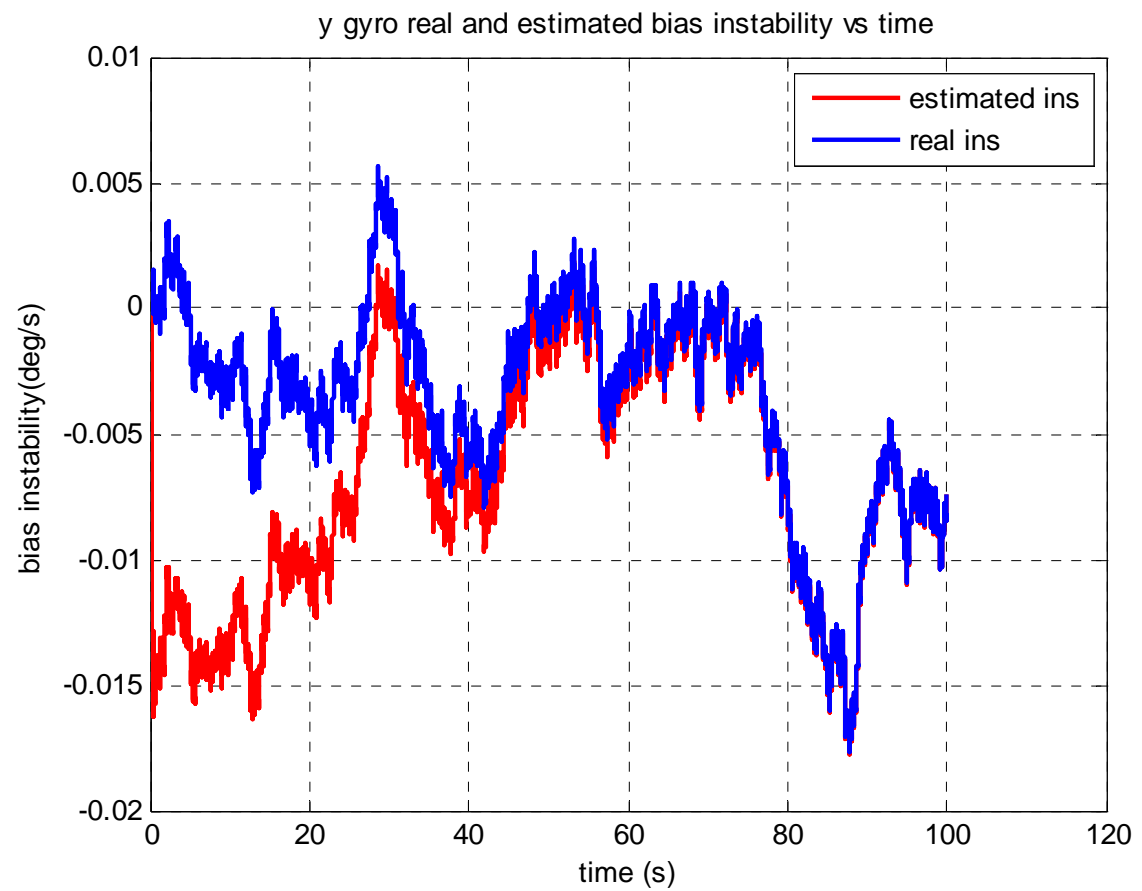

Figure 59 y gyro estimated bias instability 


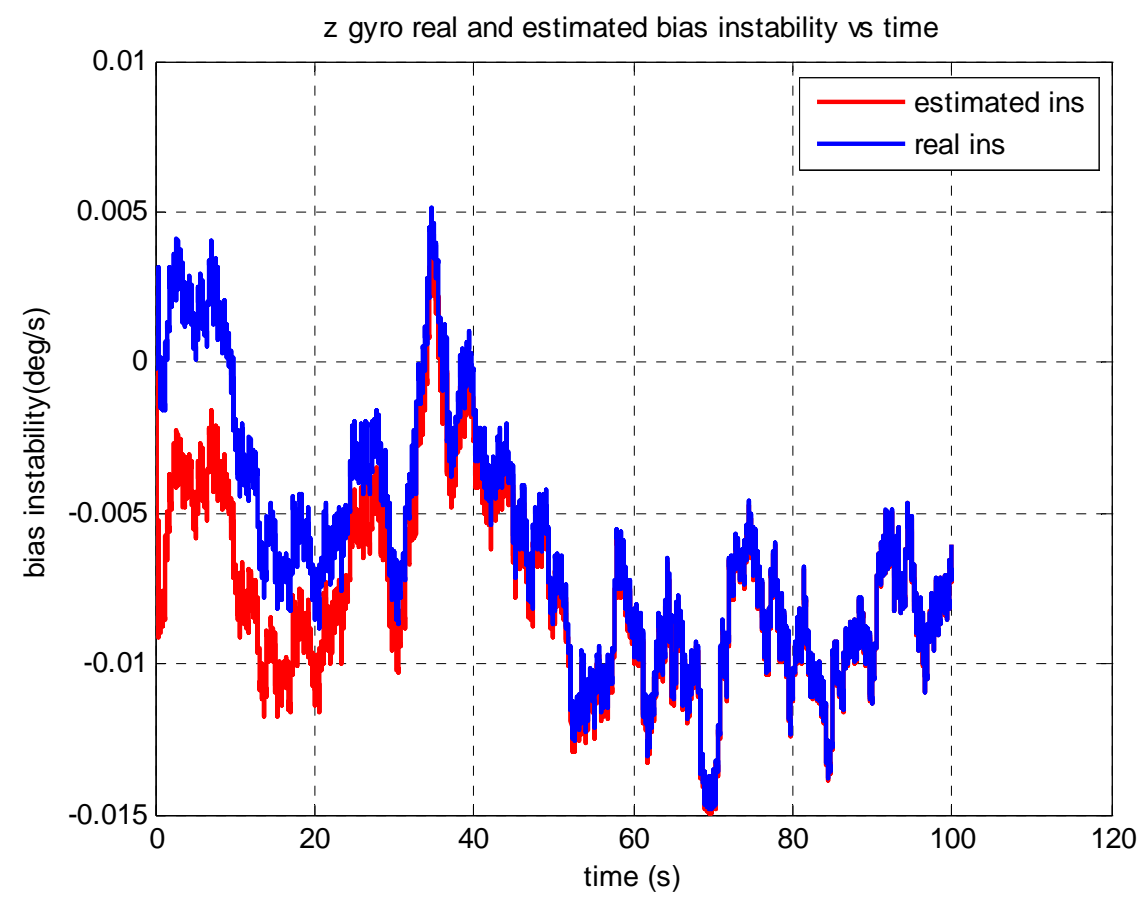

Figure 60 z gyro estimated bias instability

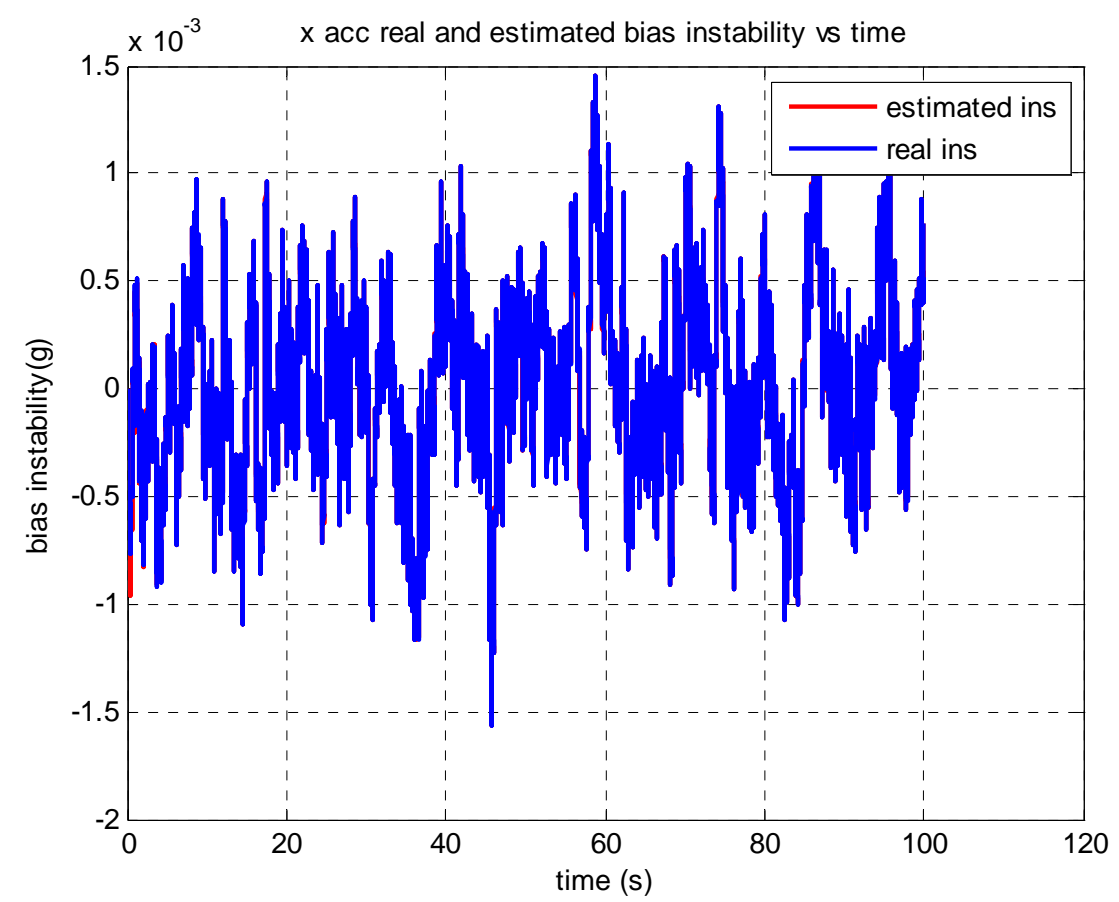

Figure $61 x$ acc estimated bias instability 


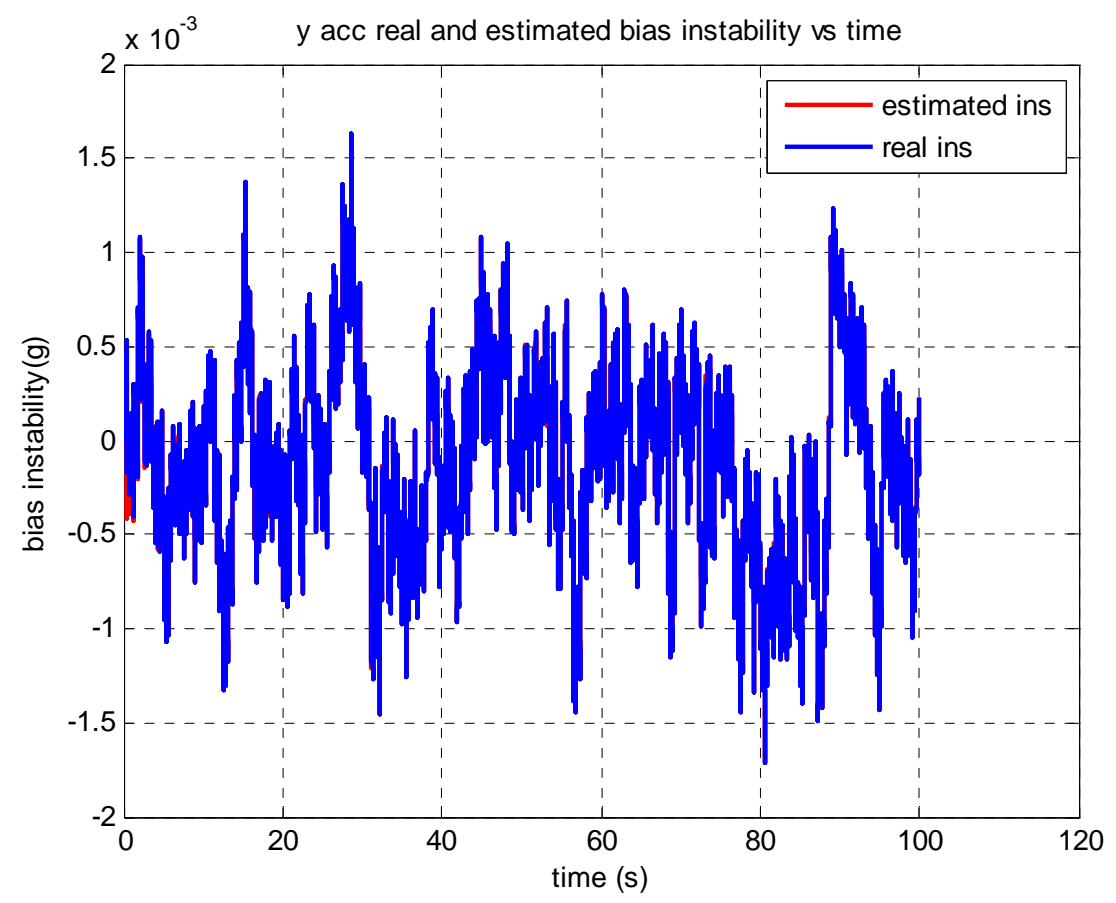

Figure 62 y acc estimated bias instability

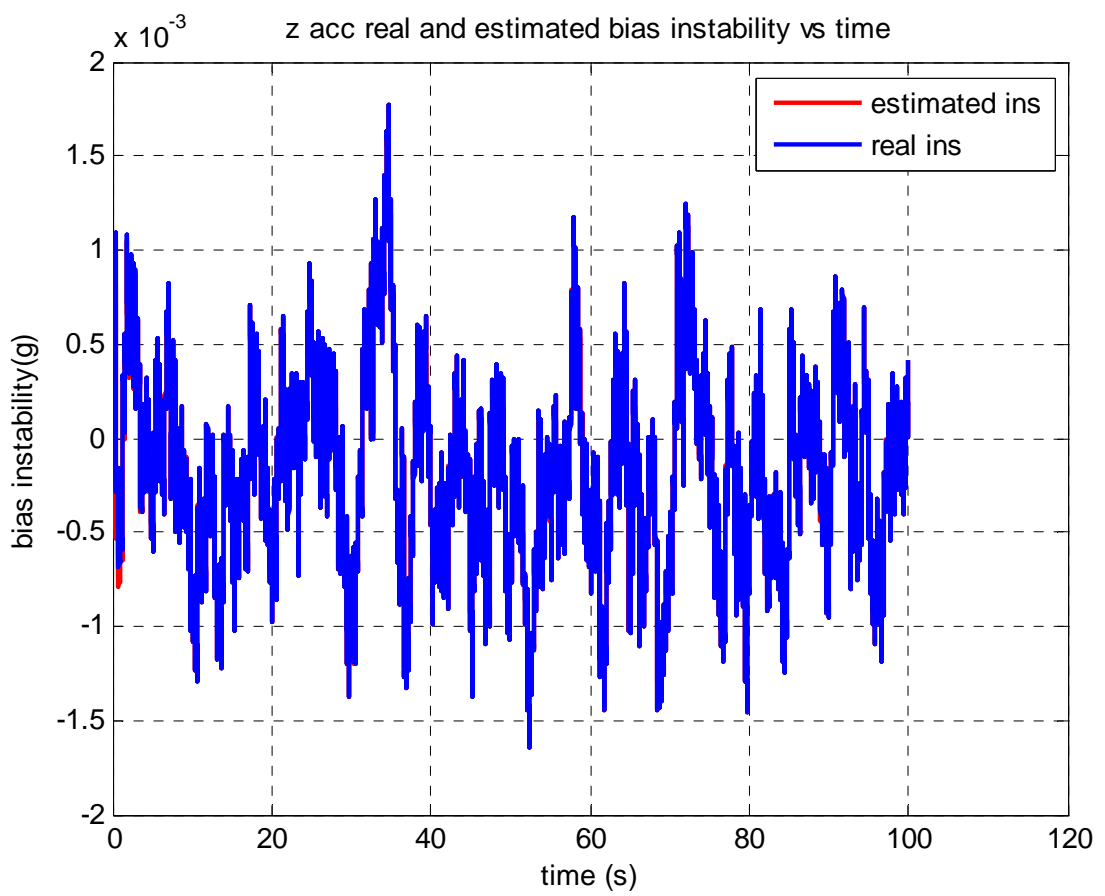

Figure 63 z acc estimated bias instability 
At the end of 4 seconds, all accelerometer bias instability estimations converged to real accelerometer bias instability values. On the other hand, convergence of gyroscope instability took 60 seconds. Gyroscope's sensor noise density and correlation time are greater than the accelerometer's noise level and correlation time. It can be observed that, the performance of estimation is directly affected by noise density and correlation time.

As mentioned earlier, random sensor noise represents the uncertainty in sensor outputs. Therefore, an increase in sensor noise level causes an increase in convergence duration. Additionally, correlation time gives information about the relationship between successive values of sensor bias instability and the relationship between consecutive values of bias instability reduces when the sensor correlation time increases. This means that, an increase in correlation time leads an increase in convergence duration.

Stochastic error estimation algorithm was processed in three different ways to observe the effects of the sensor parameters and Kalman filter components. The method which is described above, is the most basic implementation and intended to be used in practical applications. Other implementations were carried out to evaluate the effect of each parameter alone. The generated conditions for testing the effects of the parameters are listed below:

- The effect of measurement noise covariance matrix, $R$.

- The effect of sensor correlation time (time constant),

Initially, effect of measurement noise covariance matrix tuning was observed. Thus, the diagonal elements of $R$ matrix were increased and all other parameters which are listed in Table 13 and Table 14 remained the same. The revised $R$ matrix and algorithm outputs are given below: 


$$
R=\left[\begin{array}{ccccccc}
(1.422)^{2} & 0 & 0 & 0 & 0 & 0 \\
0 & (1.422)^{2} & 0 & 0 & 0 & 0 \\
0 & 0 & (1.422)^{2} & 0 & 0 & 0 \\
0 & 0 & 0 & (0.255)^{2} & 0 & 0 \\
0 & 0 & 0 & 0 & (0.255)^{2} & 0 \\
0 & 0 & 0 & 0 & 0 & (0.255)^{2}
\end{array}\right]
$$

The diagonal elements of $R$ matrix were multiplied by 10 and the algorithm was processed in this way. Results of the algorithm are given in the following figures.

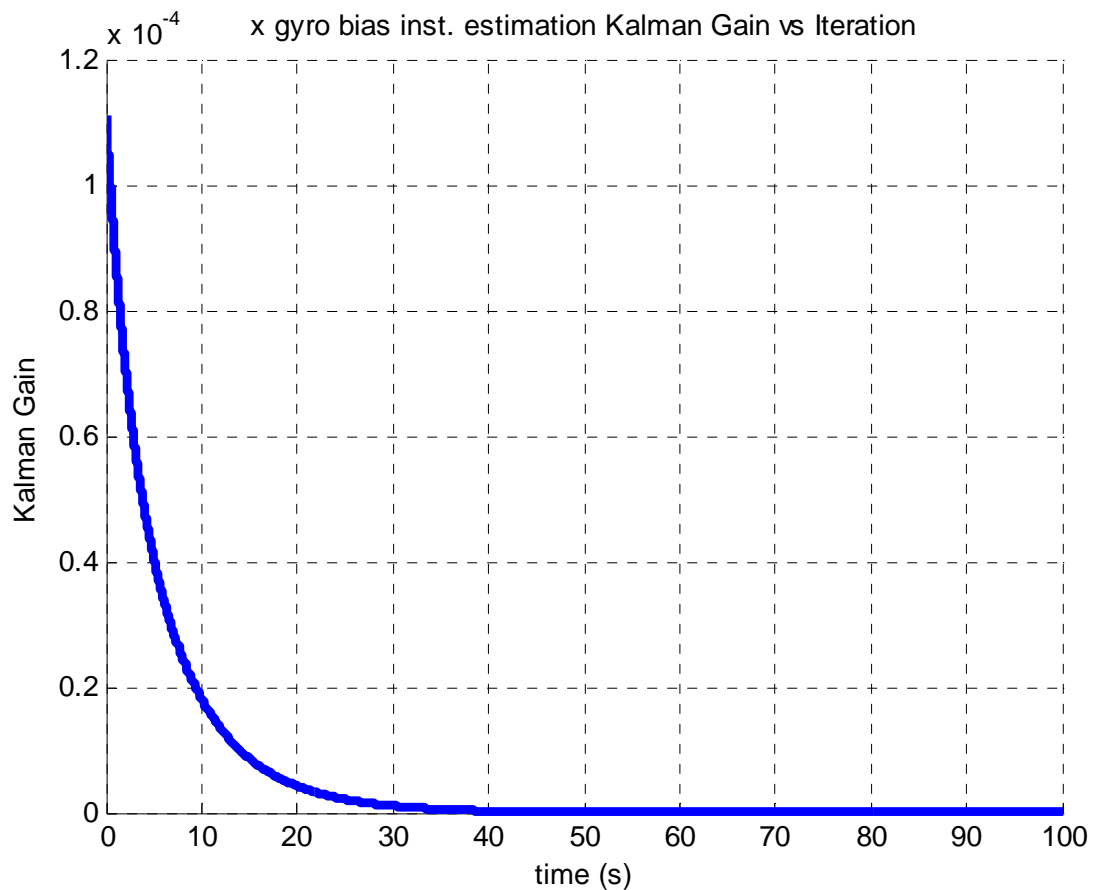

Figure 64 Gyroscpe bias instability estimation Kalman gain variation (R effect)

The Kalman gain variations for the $\mathrm{x}, \mathrm{y}, \mathrm{z}$ gyro bias instability estimations are identical. Therefore, only x gyro's Kalman gain variation is presented.

The measurement uncertainties became large with the increased measurement noise matrix. Therefore the initial value of the Kalman gain decreased, weighting the state estimates toward to their previous values[15]. 


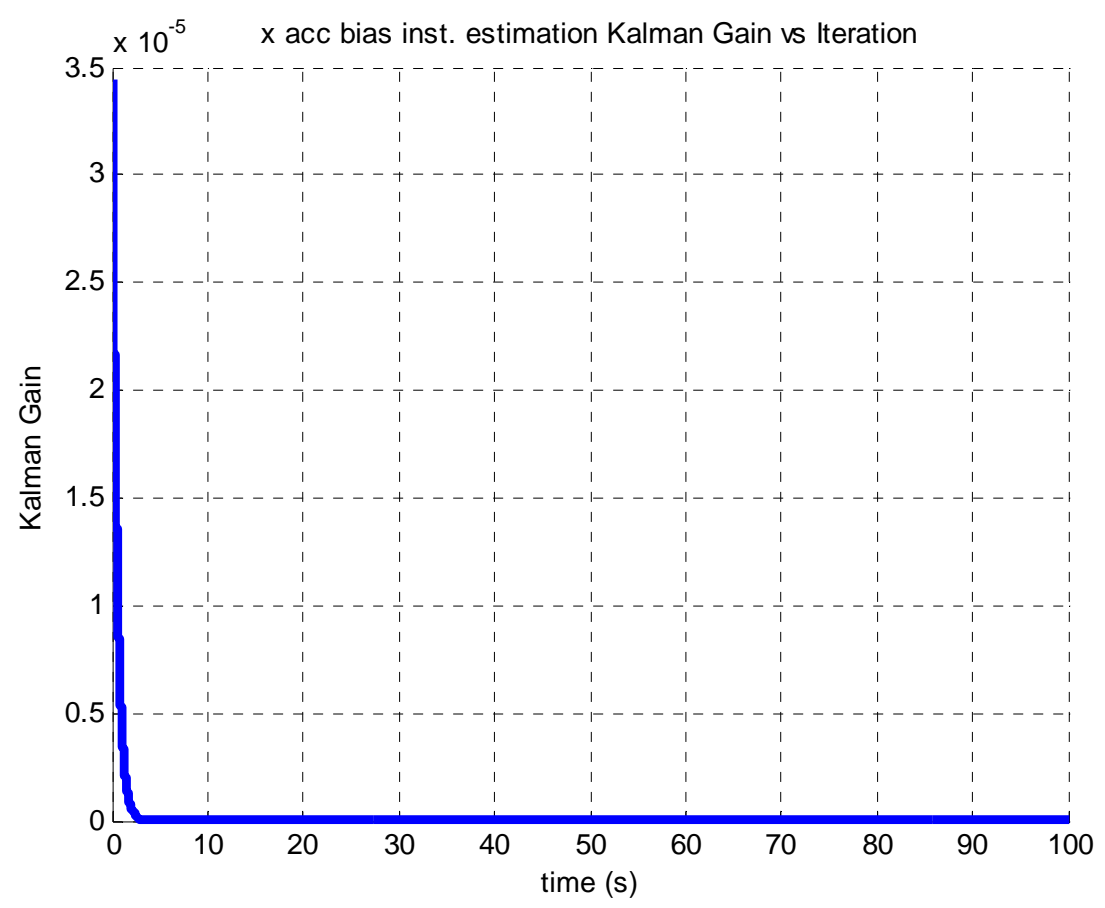

Figure 65 Accelerometer bias instability estimation Kalman gain variation ( $R$ effect)

The Kalman gain variations for the $\mathrm{x}, \mathrm{y}, \mathrm{z}$ accelerometer bias instability estimations are identical. Therefore, only x acceleromreter's Kalman gain variation is presented. 


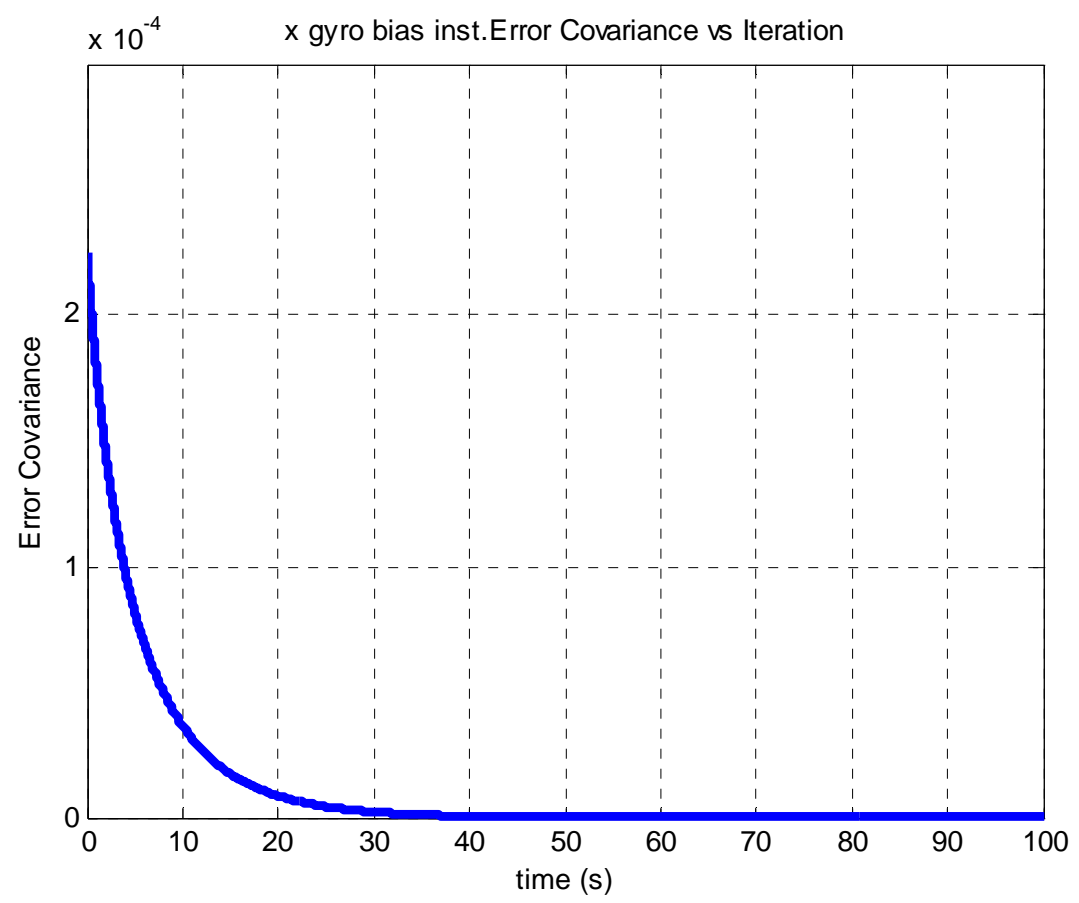

Figure 66 Gyroscope bias instability estimation error covariance variation ( $R$ effect)

The error covariance variations for the $\mathrm{x}, \mathrm{y}, \mathrm{z}$ gyro bias instability estimations are identical. Therefore, only x gyro's error covariance variation is presented. 


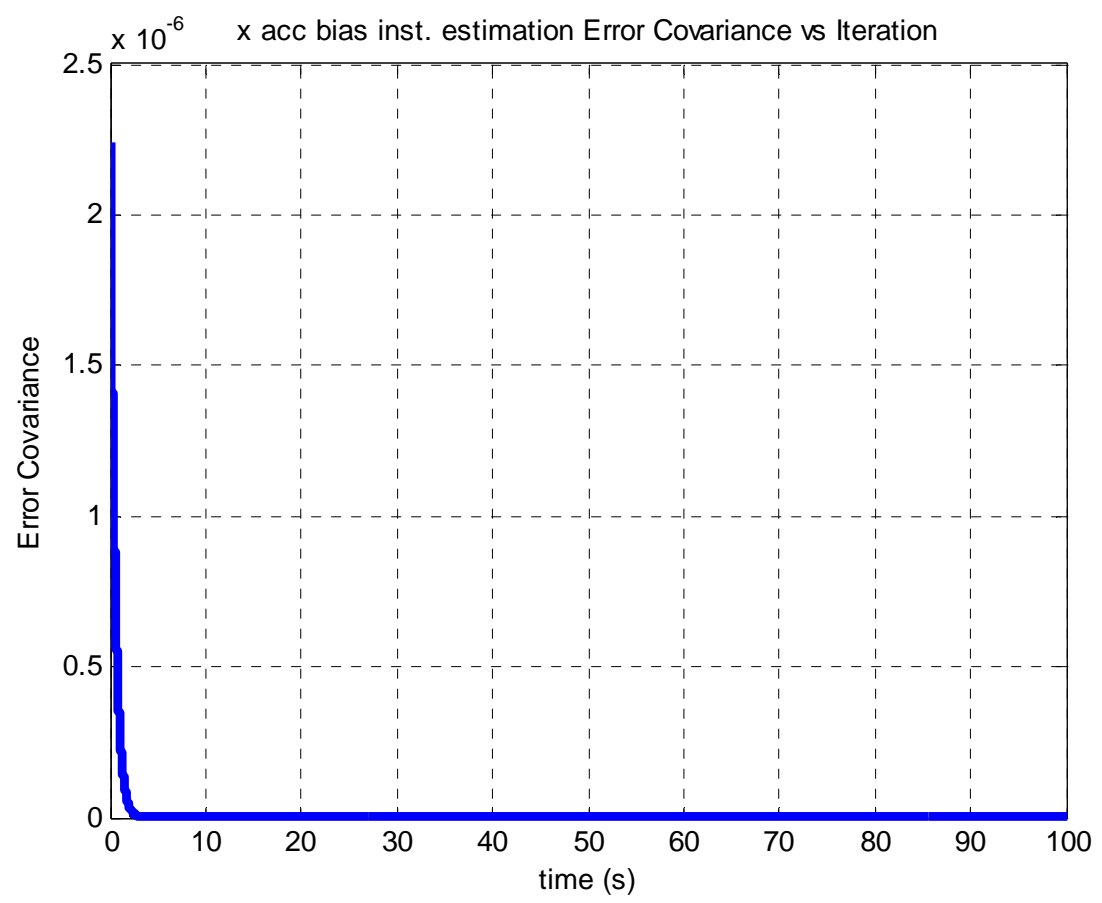

Figure 67 Accelerometer bias instability estimation error covariance variation ( $R$ effect)

The error covariance variations for the $\mathrm{x}, \mathrm{y}, \mathrm{z}$ accelerometer bias instability estimations are identical. Therefore, only x accelerometer's variation is presented. 


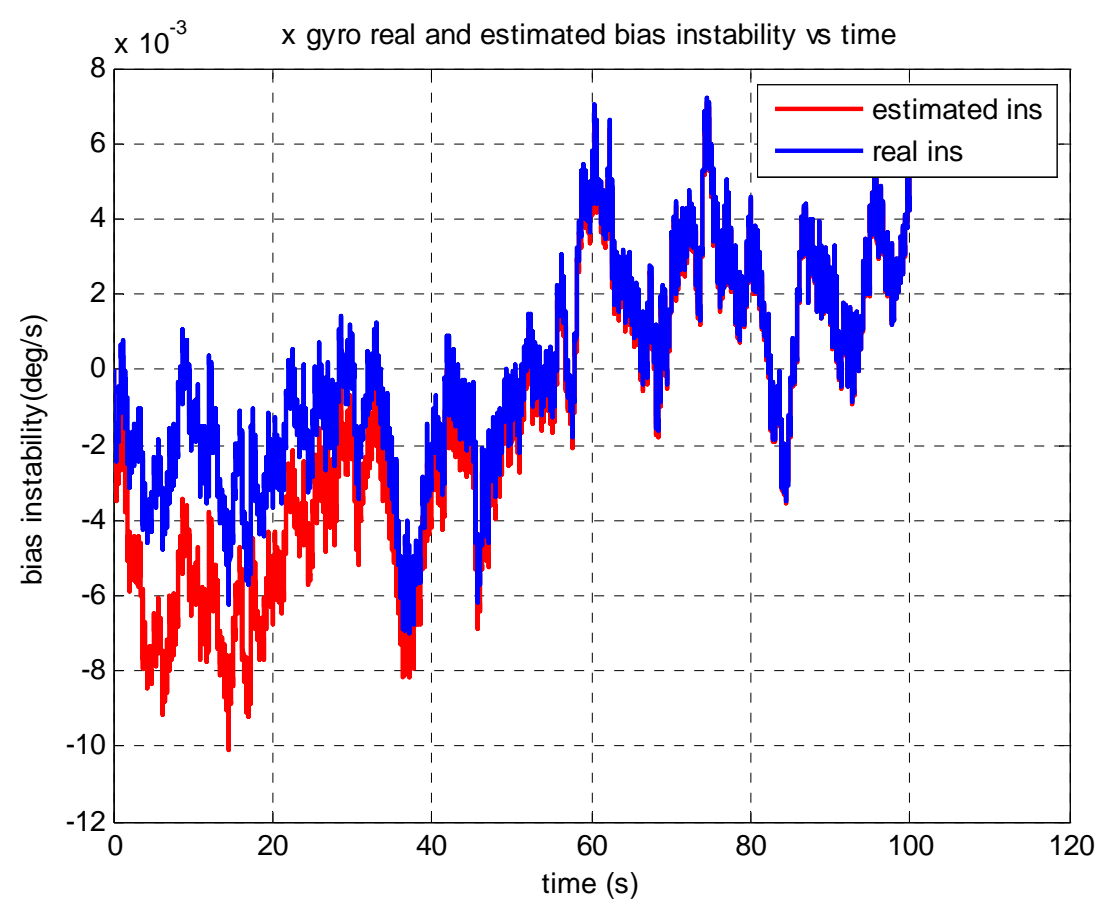

Figure $68 x$ gyro estimated bias instability ( $R$ effect)

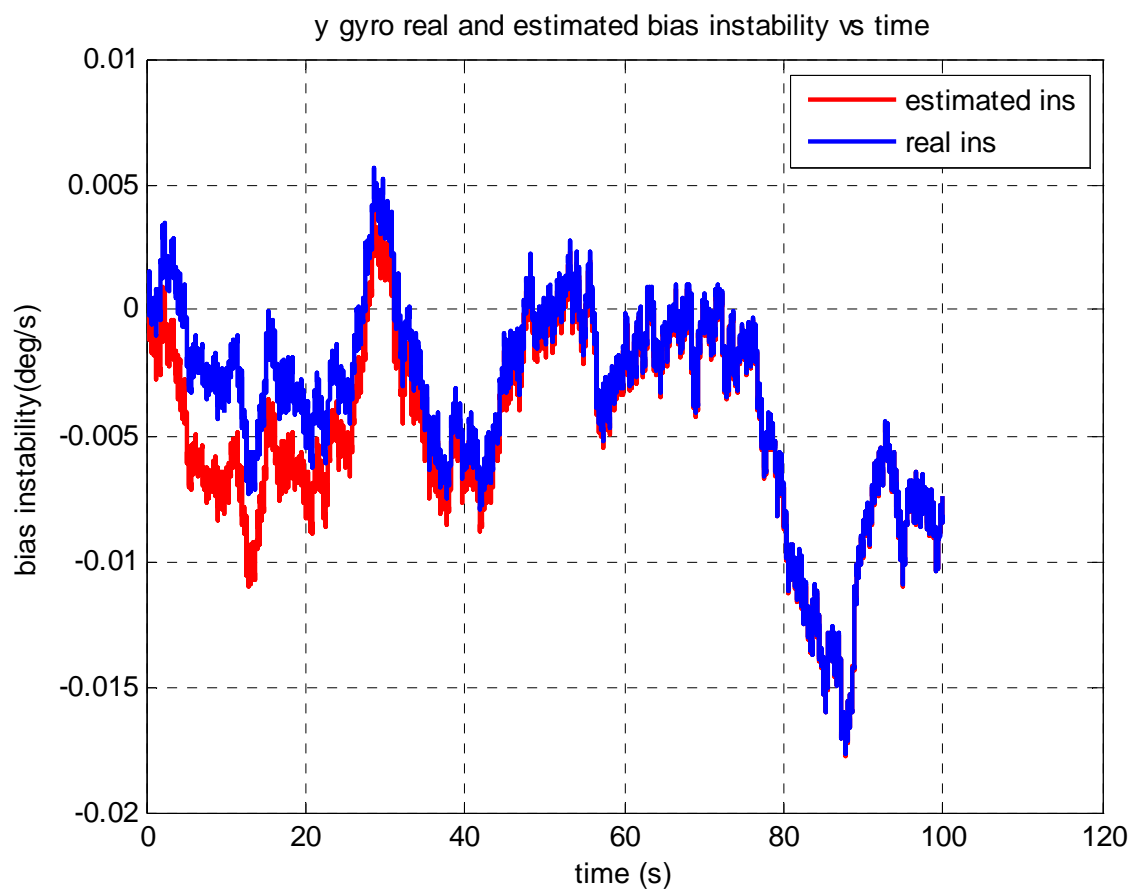

Figure 69 y gyro estimated bias instability ( $R$ effect) 


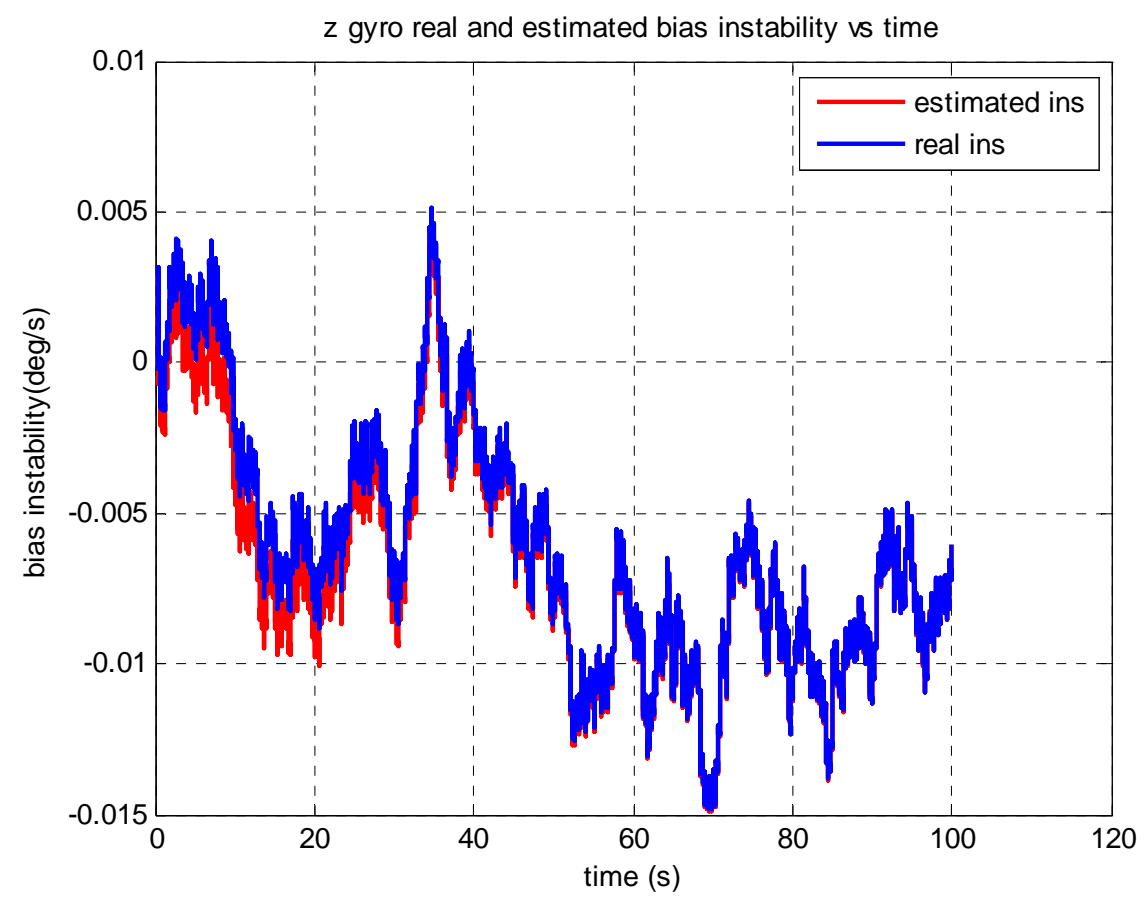

Figure 70 z gyro estimated bias instability ( $R$ effect)

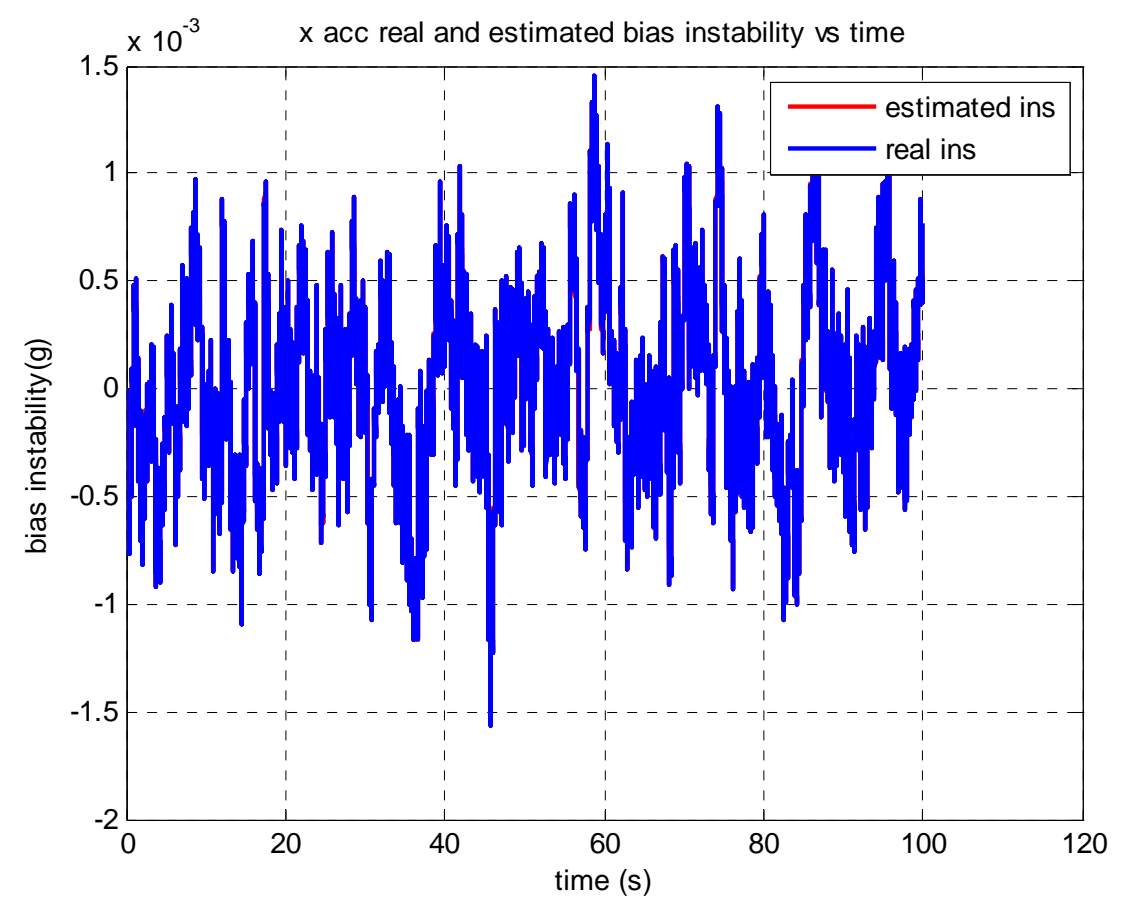

Figure $71 x$ acc estimated bias instability ( $R$ effect) 


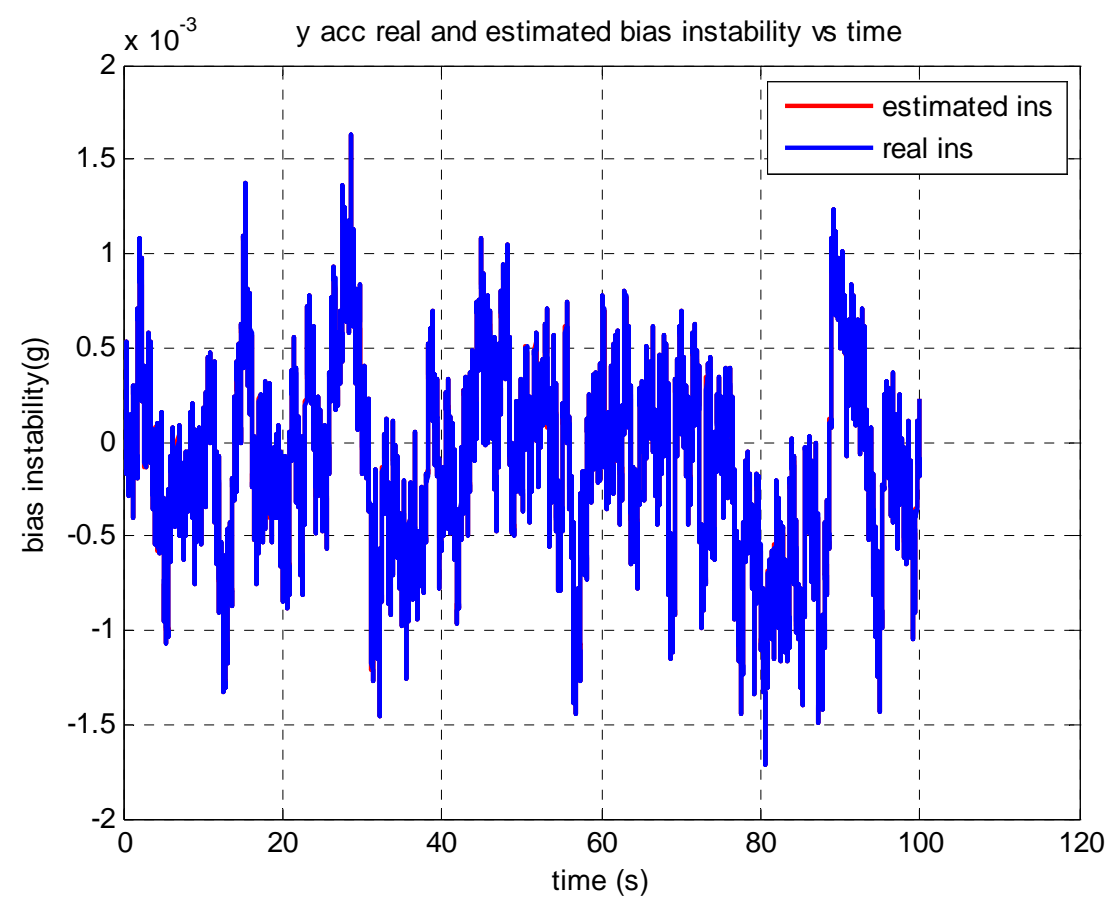

Figure 72 y acc estimated bias instability ( $R$ effect)

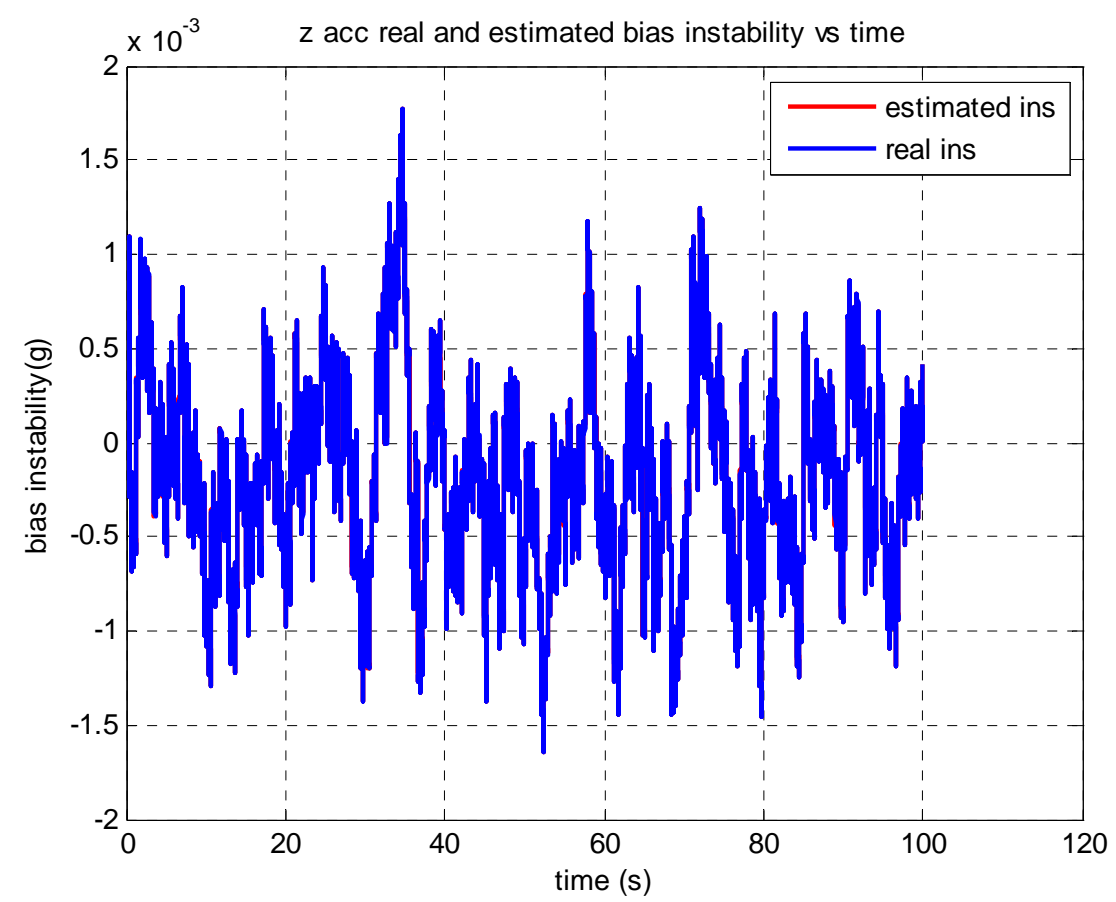

Figure 73 z acc estimated bias instability ( $R$ effect) 
At the end of 1 second, all accelerometer bias instability estimations converged to real accelerometer bias instability values. On the other hand, convergence of gyroscope instability took 40 seconds.

The uncertainties in measurements become large with the increase in measurement noise covariance. As a result of this, the Kalman gain decrease to maintain the performance of the estimation.

As obtained from the algorithm results rate of convergence increases when the measurement noise covariance increases. It means that the estimations of accelerometer and gyroscope bias instabilities can reach the true values of accelerometer and gyroscope bias instabilities more quickly. Because, increase in measurement noise covariance causes the Kalman gain to drop, weighting the state estimates more toward their previous states [15].

Secondly, the effect of sensor correlation time on the algorithm performance was investigated. In order to investigate the effect of sensor correlation time, stochastic properties of accelerometers and gyroscopes were changed. Correlation time of the accelerometers was increased from 1 second to 10 seconds and correlation time of the gyroscopes was also increased from 15 seconds to 30 seconds. Additionally, simulation duration was raised to 200 seconds. Other parameters which are listed in Table 13, Table 14 and equation (7.3) remained the same.

When the algorithm was processed for the first time, duration was set to 100 seconds. However, estimated accelerometer and gyroscope bias instabilities could not converge to real instabilities. Therefore duration of simulation was increased to 200 seconds.

The following figures show that the sensor correlation time is one of the major components for algorithm design. Because the length of correlation time directly affects the convergence time. With the increase of correlation time, occurrence of bias instability takes more time. Besides, high correlation time represents that the 
successive values of sensor outputs are weakly correlated. Therefore, the performance of the estimation algorithm decreased and convergence happened later. Consequently, it can be said that if the sensor has a high correlation time, stability performance of the sensor is good and the sensor does not need any instability estimation and correction algorithm. For example, correlation time of ring laser gyroscopes is approximately 1000 seconds and the IMUs which contain ring laser gyroscopes do not use bias instability estimation algorithm. Outputs of the algorithm are given in the following figures.

All estimated stochastic error parameters oscillates between $-3 \sigma$ and $+3 \sigma$ values of the bias instabilities. This result indicates that the estimation algorithm works properly.

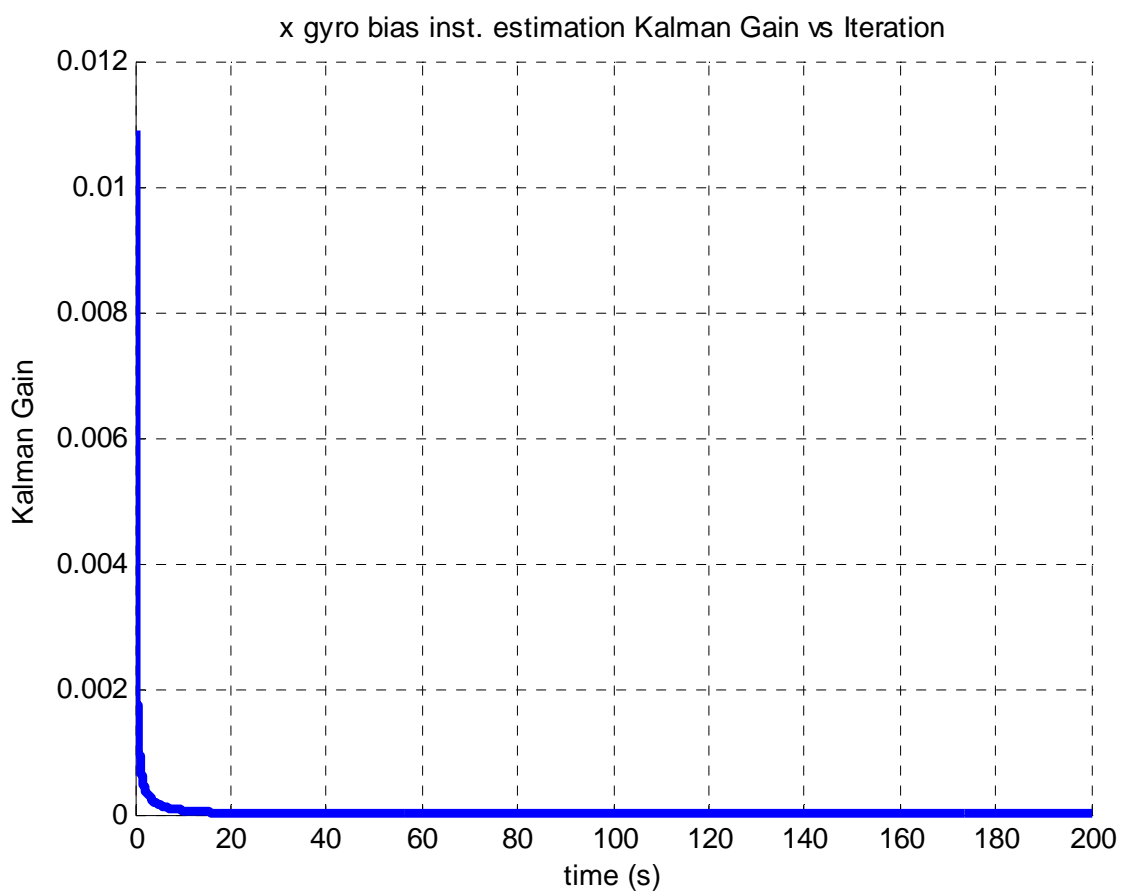

Figure 74 Gyroscope bias instability estimation Kalman gain variation (correlation time effect)

The Kalman gain variations for the $\mathrm{x}, \mathrm{y}, \mathrm{z}$ gyro bias instability estimations are identical. Therefore, only x gyro's Kalman gain variation is presented. 


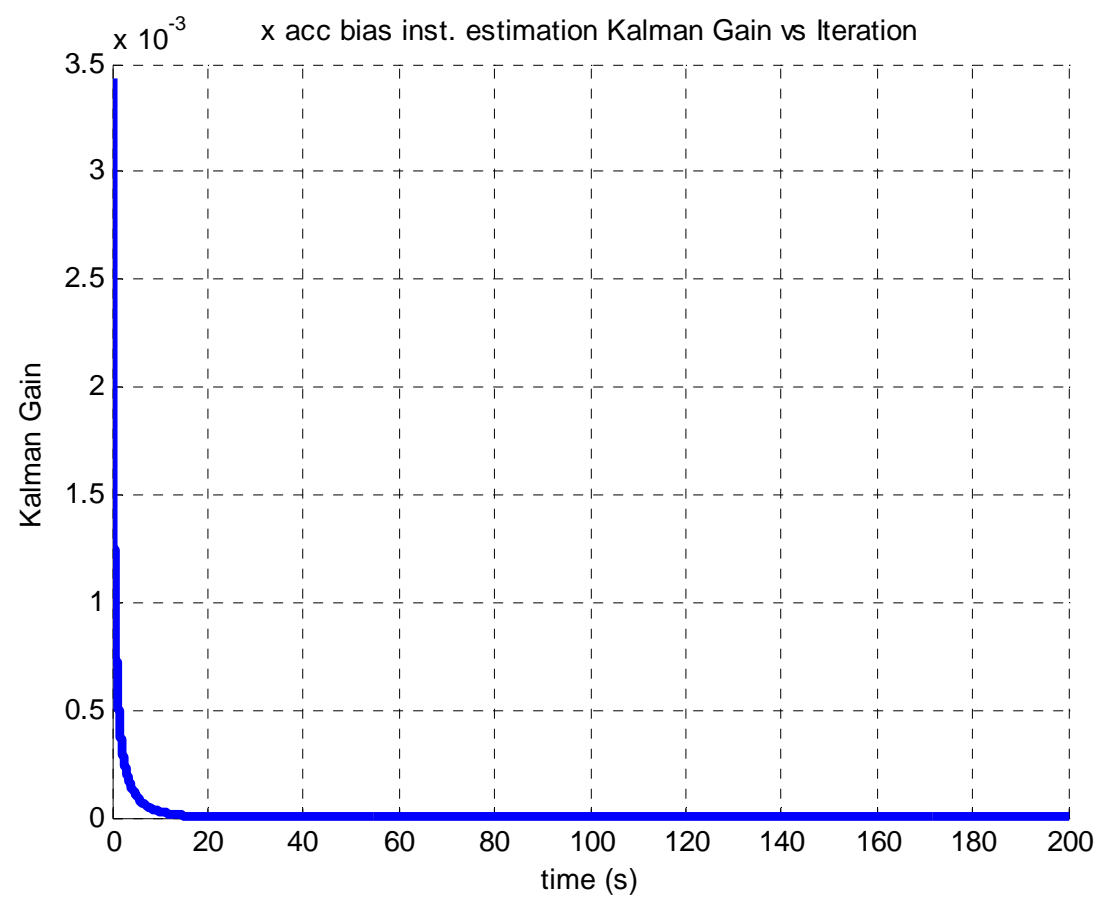

Figure 75 Accelerometer bias instability estimation Kalman gain variation (correlation time effect)

The Kalman gain variations for the $\mathrm{x}, \mathrm{y}, \mathrm{z}$ accelerometer bias instability estimations are identical. Therefore, only $\mathrm{x}$ accelerometer's Kalman gain variation is presented. 


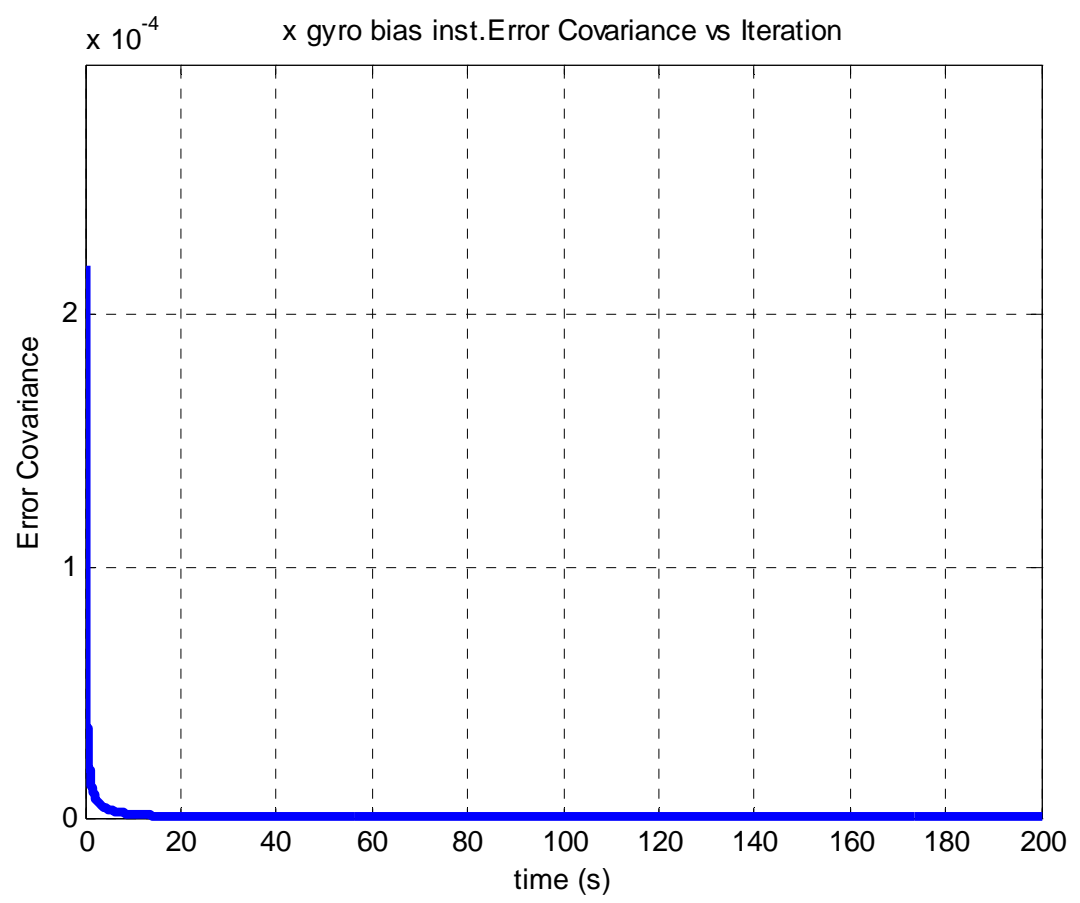

Figure 76 Gyroscope bias instability estimation error covariance variation (correlation time effect)

The error covariance variations for the $\mathrm{x}, \mathrm{y}, \mathrm{z}$ gyro bias instability estimations are identical. Therefore, only x gyro's error covariance variation is presented. 


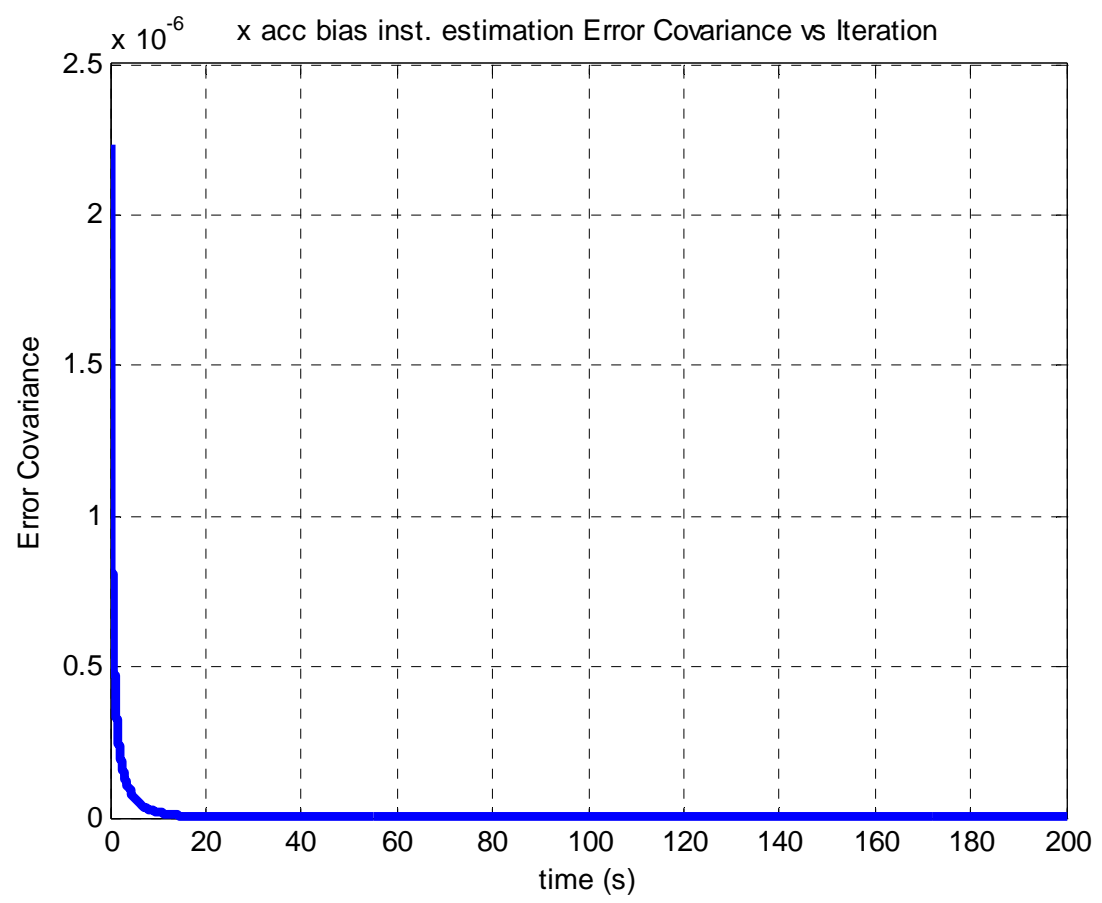

Figure 77 Accelerometer bias instability estimation error covariance variation (correlation time effect)

The error covariance variations for the $\mathrm{x}, \mathrm{y}, \mathrm{z}$ accelerometer bias instability estimations are identical. Therefore, only x accelerometer's variation is presented. 

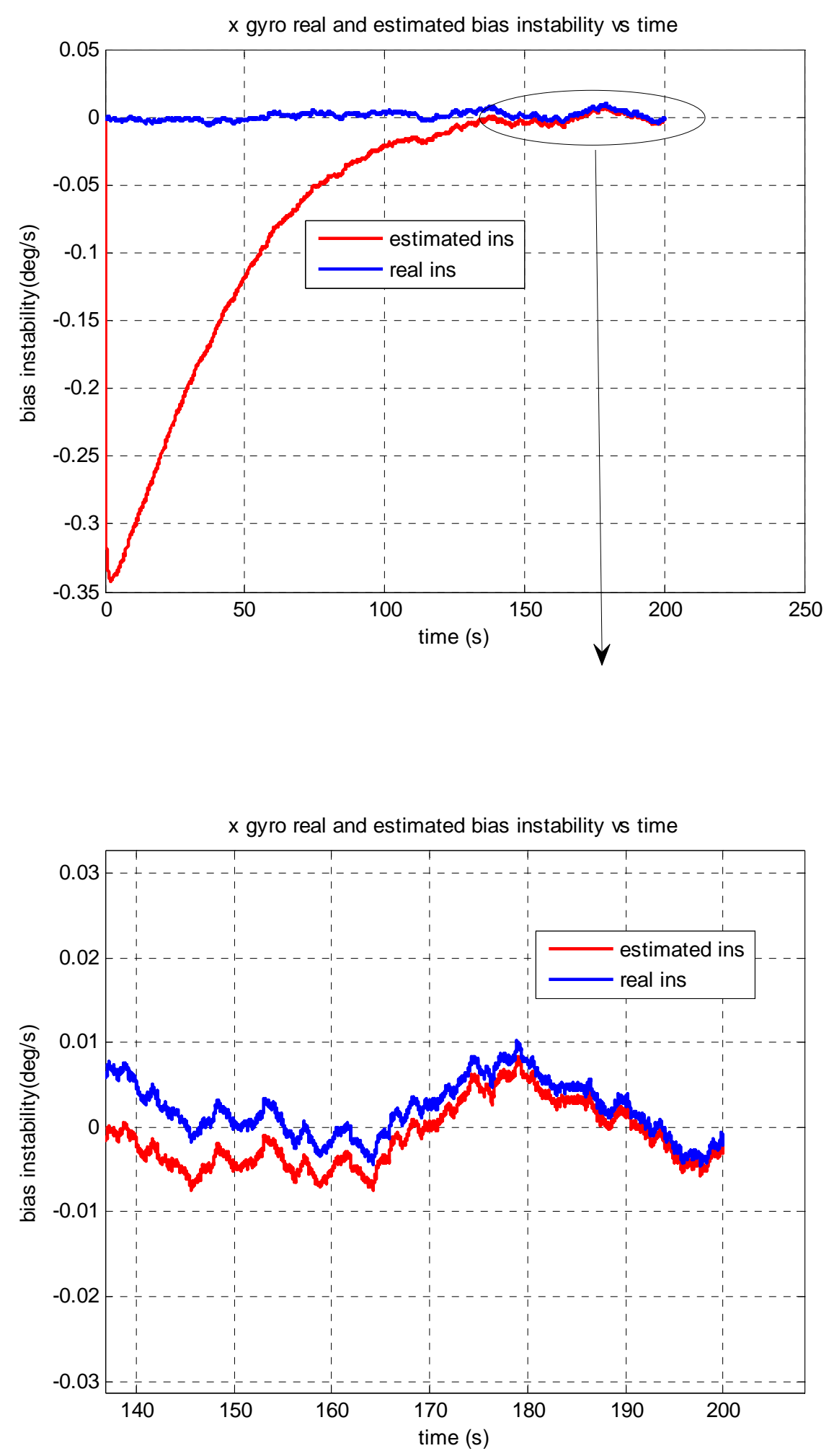

Figure $78 x$ gyro estimated bias instability (correlation time effect) 

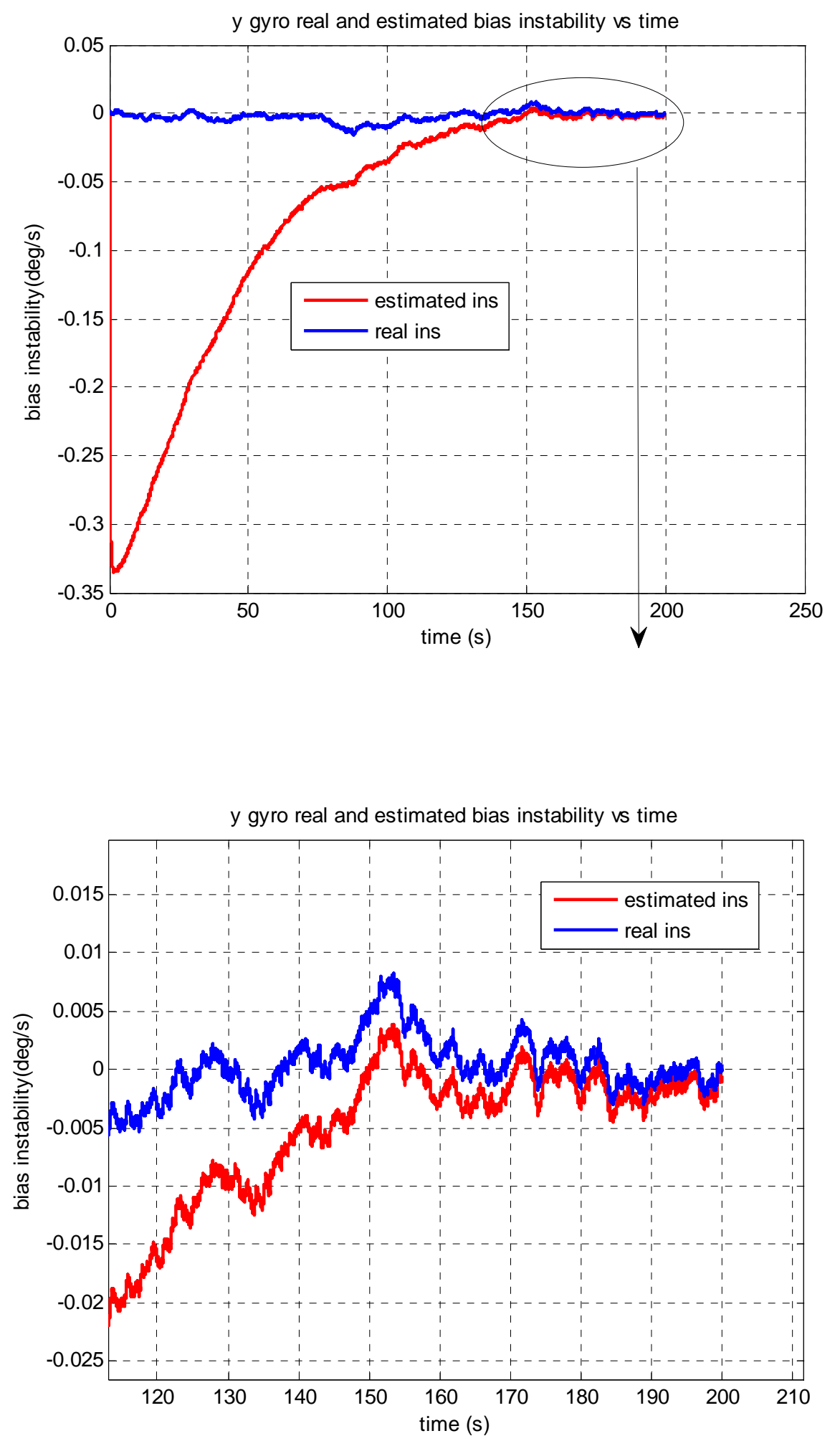

Figure 79 y gyro estimated bias instability (correlation time effect) 

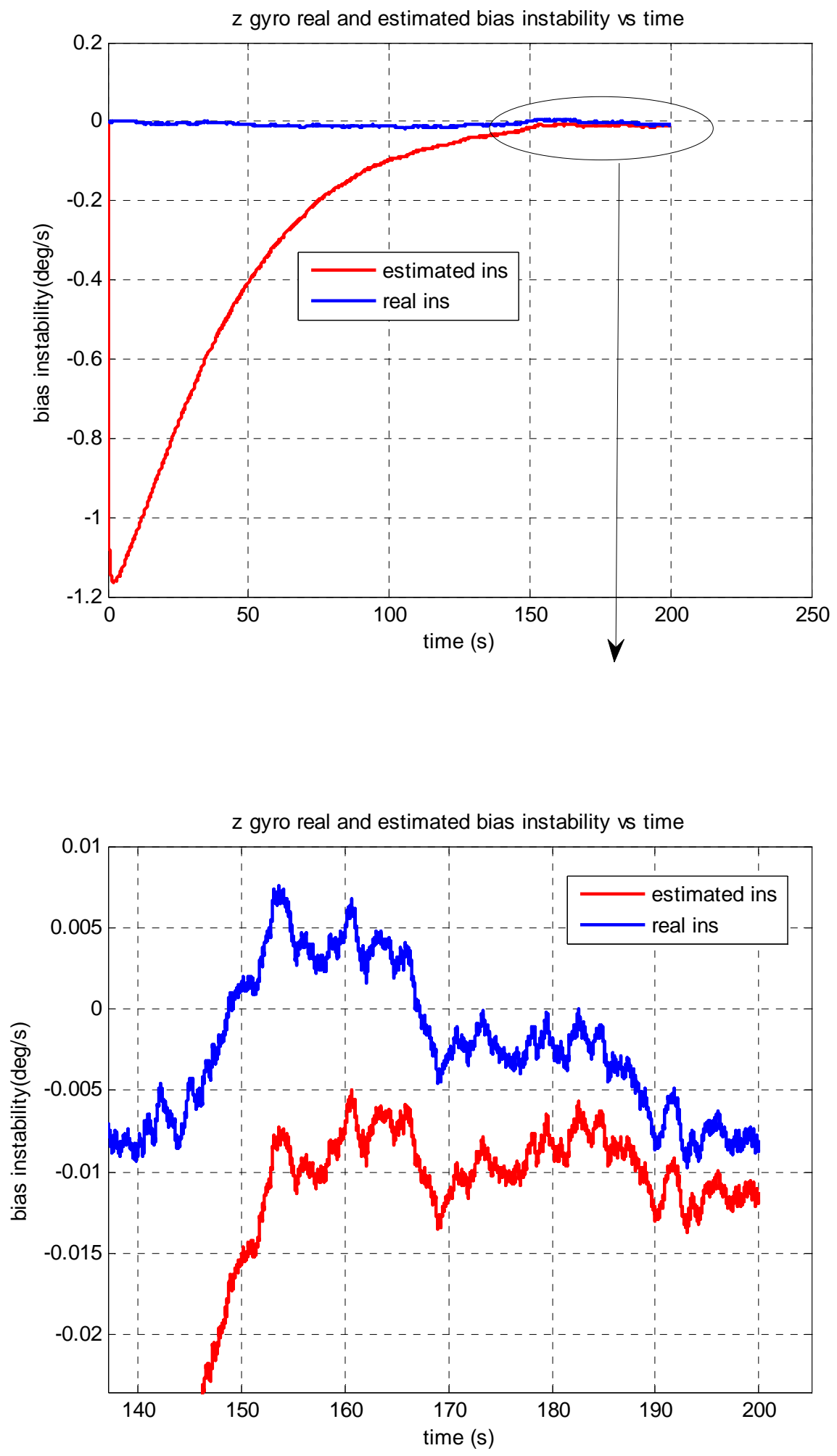

Figure 80 z gyro estimated bias instability (correlation time effect) 

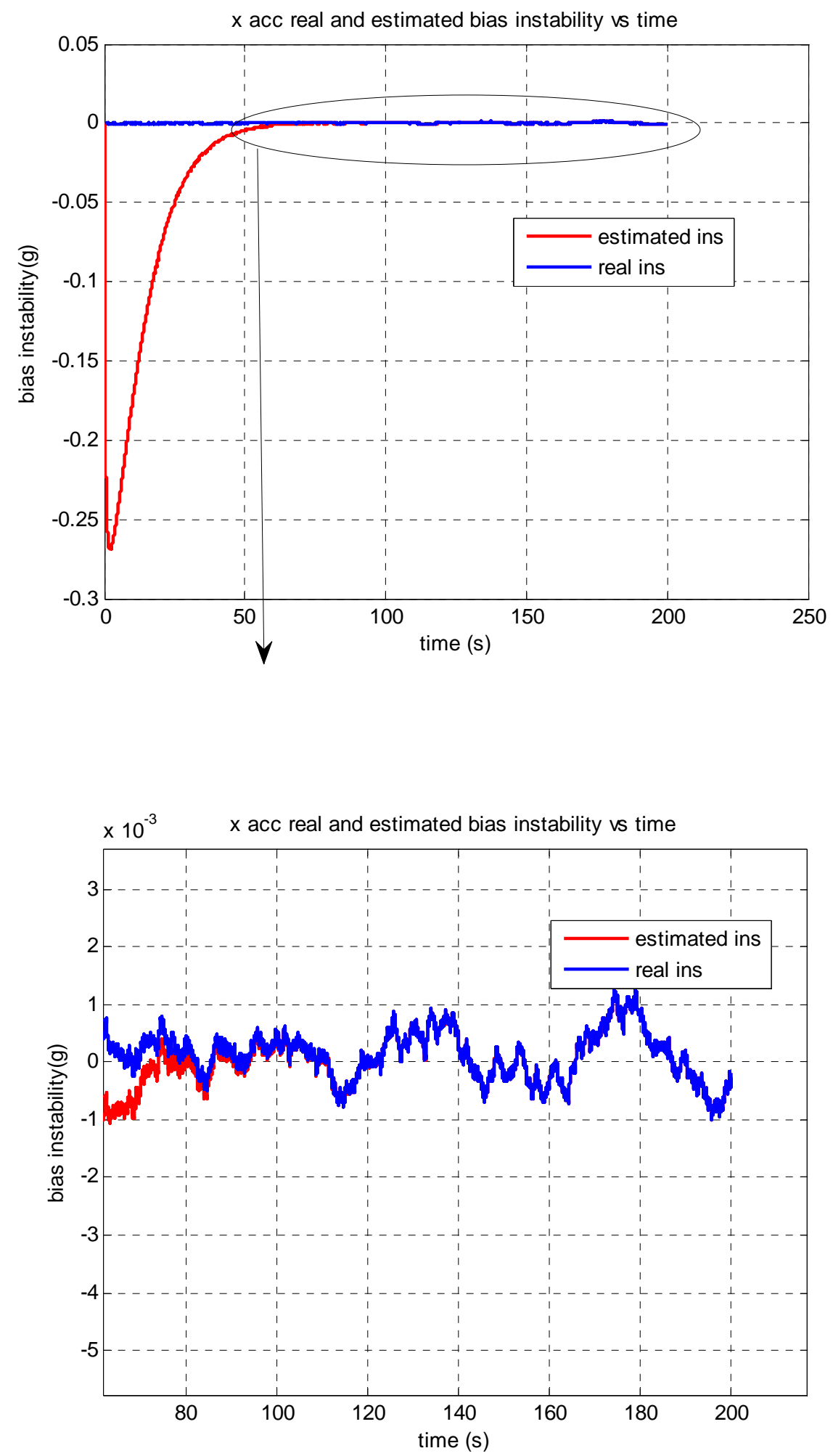

Figure $81 x$ acc estimated bias instability (correlation time effect) 

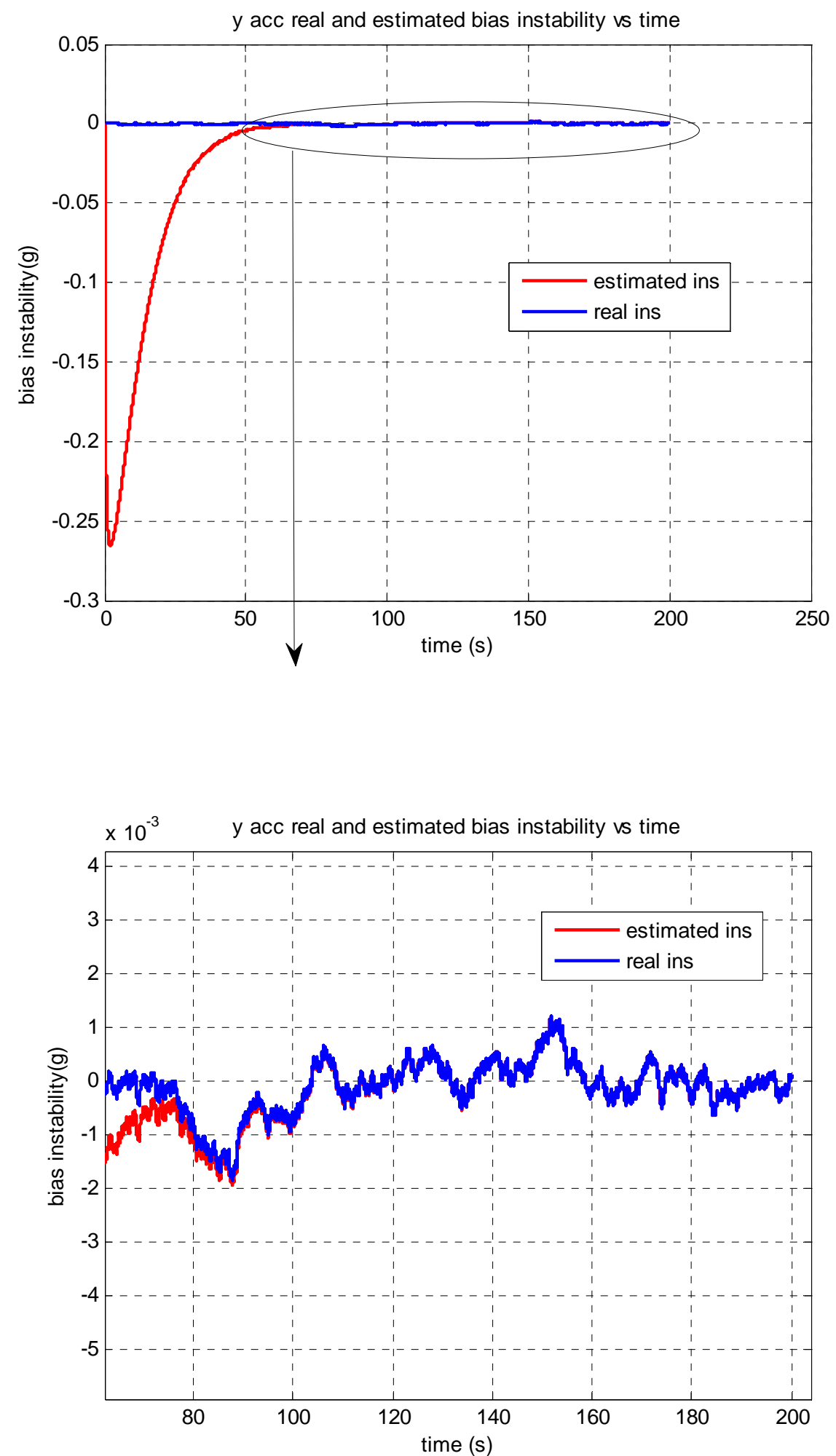

Figure 82 y acc estimated bias instability (correlation time effect) 

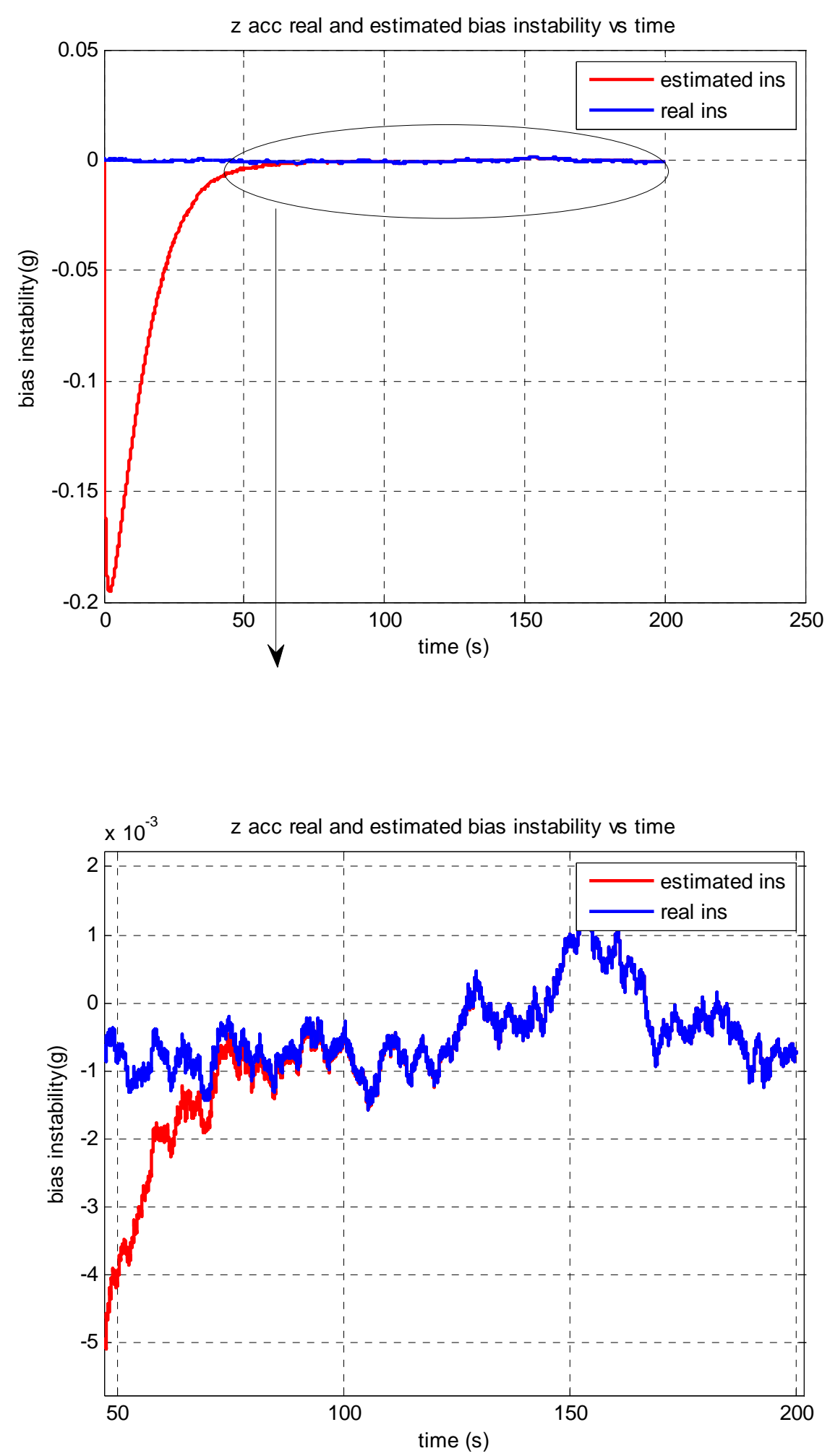

Figure 83 z acc estimated bias instability (correlation time effect) 


\subsection{Experimental Test Results}

A tactical grade IMU was used to perform real experiments. The commercial IMU which was used in the experiments contains three MEMS Colibrys MS9000.D series accelerometer and three identical ADIS16100 MEMS gyroscope. Additionally, the IMU has a processing unit for running deterministic error compensation algorithm.

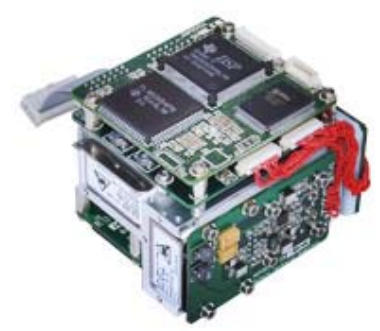

Figure 84 Representation of MEMS IMU

Multi-position static test and multi-rate dynamic test were performed respectively. The IMU data which was collected during the multi-position static test and multi-rate dynamic test were used to estimate deterministic errors of the IMU.

Acutronic 3-axis rate table was used to simulate reference position and angular rate. In addition, test computer and power supply were used in the experimental tests. Test setup is presented in Figure 85.

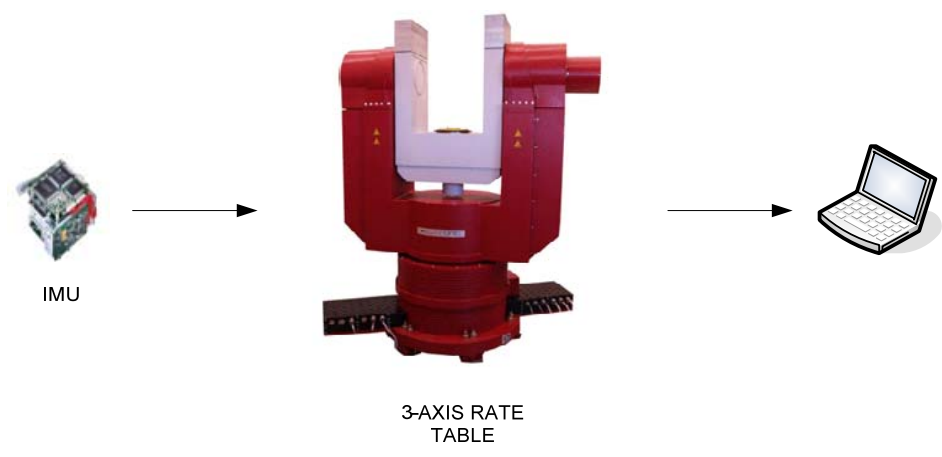

Figure 85 Experimental test setup 
Deterministic error parameters of the IMU were estimated by processing the collected test data accordingly deterministic error estimation algorithm. Estimated error parameters are given in Table-15. Error compensation (calibration) simulation was run by using estimated deterministic error parameters and calibrated IMU outputs were acquired.

Table 15Deterministic Error Estimation Results (Real data)

\begin{tabular}{|c|c|c|c|}
\hline Gyro & $\begin{array}{l}x \\
\text { estimated }\end{array}$ & $\begin{array}{l}\mathbf{y} \\
\text { estimated }\end{array}$ & $\begin{array}{l}z \\
\text { estimated }\end{array}$ \\
\hline Bias & $\begin{array}{c}1,7507 \\
\mathrm{deg} / \mathrm{s}\end{array}$ & $\begin{array}{c}0,1589 \\
\mathrm{deg} / \mathrm{s}\end{array}$ & $\begin{array}{c}1,5544 \\
\mathrm{deg} / \mathrm{s}\end{array}$ \\
\hline $\begin{array}{l}\text { G-dep bias } \\
\text { Coefficient }\end{array}$ & $\begin{array}{l}0.0365 \\
\mathrm{deg} / \mathrm{s} / \mathrm{g}\end{array}$ & $\begin{array}{l}0.0389 \\
\mathrm{deg} / \mathrm{s} / \mathrm{g}\end{array}$ & $\begin{array}{l}0.0101 \\
\mathrm{deg} / \mathrm{s} / \mathrm{g}\end{array}$ \\
\hline $\begin{array}{l}\text { Scale Factor } \\
\text { Error }\end{array}$ & $\begin{array}{l}6900 \\
\text { ppm }\end{array}$ & $\begin{array}{l}5112 \\
\text { ppm }\end{array}$ & $\begin{array}{c}10685 \\
\mathrm{ppm}\end{array}$ \\
\hline Misalignment & $\begin{array}{c}\text { xy } 9.7416 \\
\text { mrad } \\
\text { xz } 9.1504 \\
\text { mrad }\end{array}$ & $\begin{array}{c}\text { yx } 6.5824 \\
\text { mrad } \\
\text { yz } 6.4949 \\
\text { mrad }\end{array}$ & $\begin{array}{c}\text { zx } 2.9288 \\
\text { mrad } \\
\text { zy } 1.3613 \\
\text { mrad }\end{array}$ \\
\hline Accelerometer & & & \\
\hline Bias & $\begin{array}{c}262.6618 \\
\mathrm{mg}\end{array}$ & $\begin{array}{c}203.9583 \\
\mathrm{mg}\end{array}$ & $\begin{array}{c}394.0867 \\
\mathrm{mg}\end{array}$ \\
\hline $\begin{array}{l}\text { Scale Factor } \\
\text { Error }\end{array}$ & $\begin{array}{l}7771 \\
\mathrm{ppm}\end{array}$ & $\begin{array}{l}5734 \\
\text { ppm }\end{array}$ & $\begin{array}{c}13.3880 \\
\text { ppm }\end{array}$ \\
\hline Misalignment & $\begin{array}{c}\text { xy } 1.4531 \\
\text { mrad } \\
\text { xz } 2.2908 \\
\text { mrad }\end{array}$ & $\begin{array}{c}\mathrm{yx} 4.9527 \\
\mathrm{mrad} \\
\mathrm{yz} 7.9273 \\
\mathrm{mrad}\end{array}$ & $\begin{array}{c}\mathrm{zx} 1.4983 \\
\mathrm{mrad}\end{array}$ \\
\hline
\end{tabular}

Collected raw IMU data and calibrated IMU data is given in the following figures. It can be observed that the error compensation (calibration) model outputs oscillate around the reference values. 


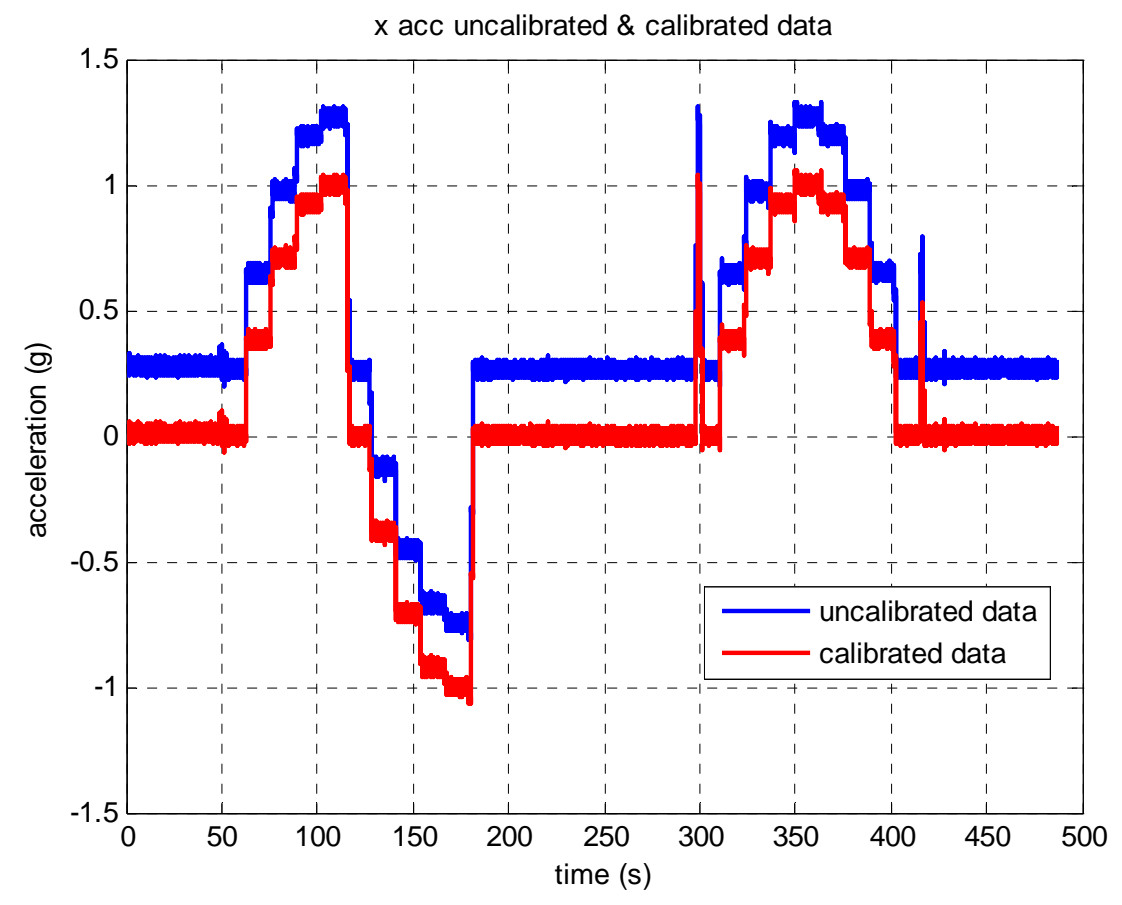

Figure 86 uncalibrated and calibrated $x$ acc output (real data)

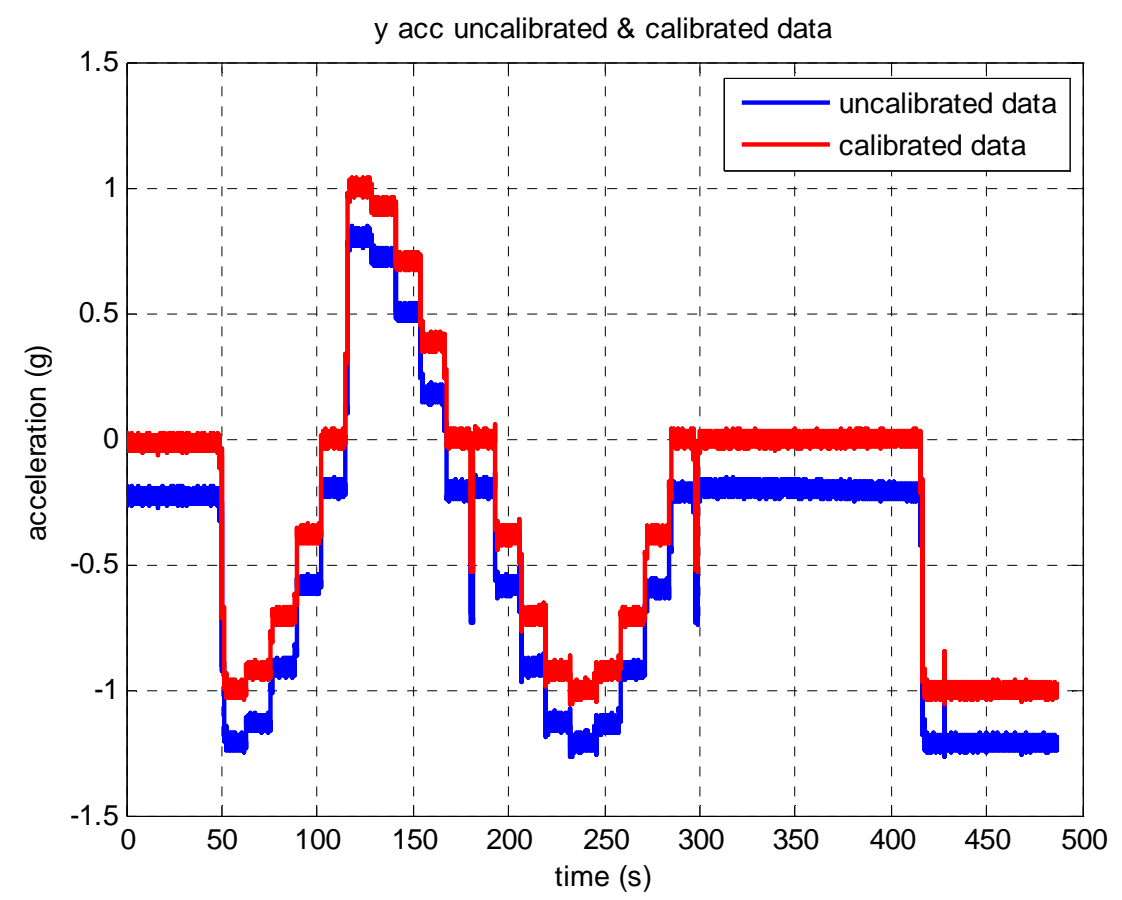

Figure 87 uncalibrated and calibrated y acc output (real data) 


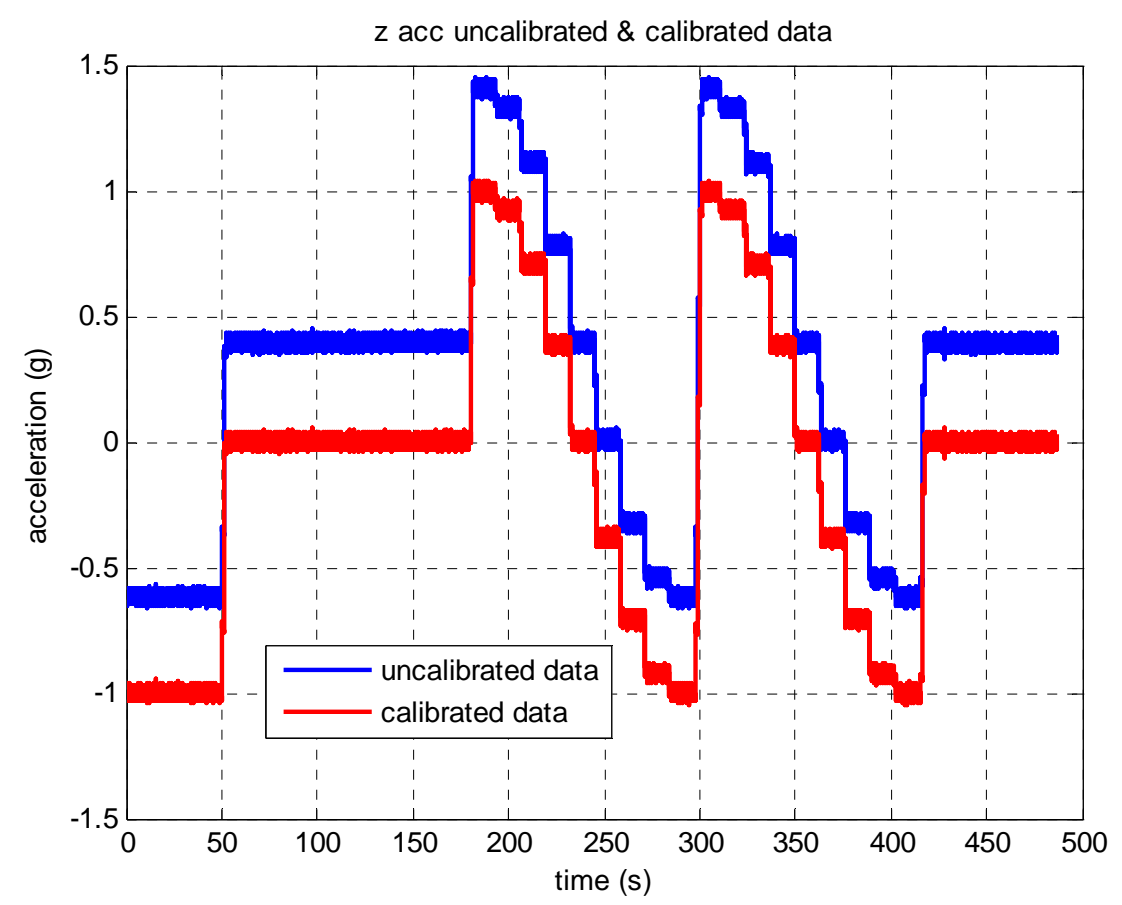

Figure 88 uncalibrated and calibrated $z$ acc output (real data)

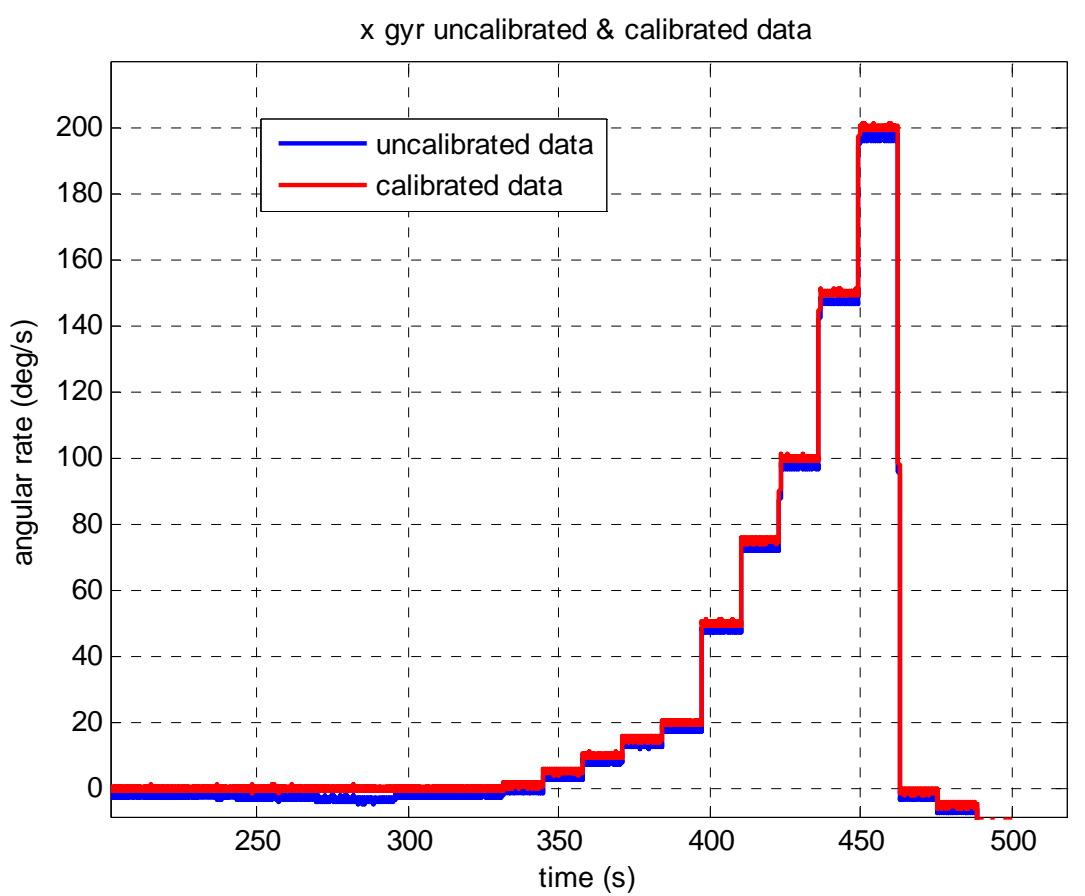

Figure 89 uncalibrated and calibrated $x$ gyro output (real data) 


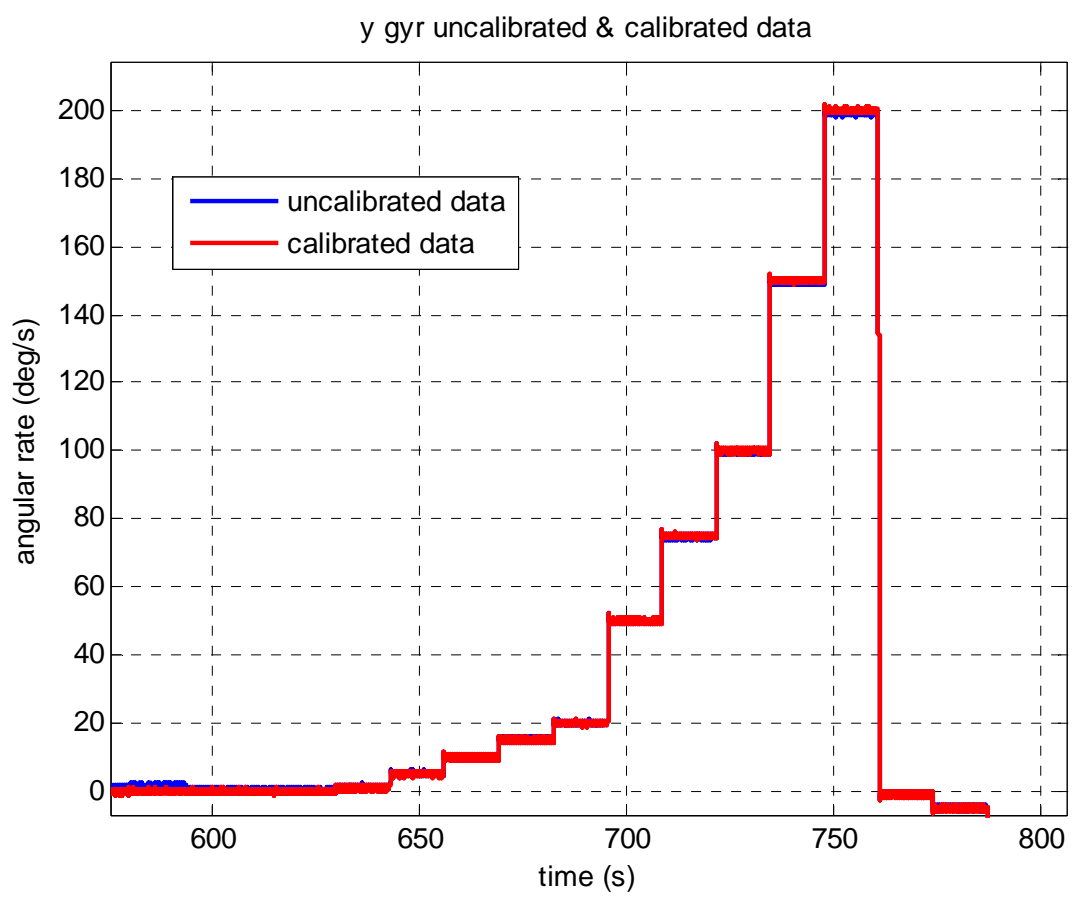

Figure 90 uncalibrated and calibrated y gyro output (real data)

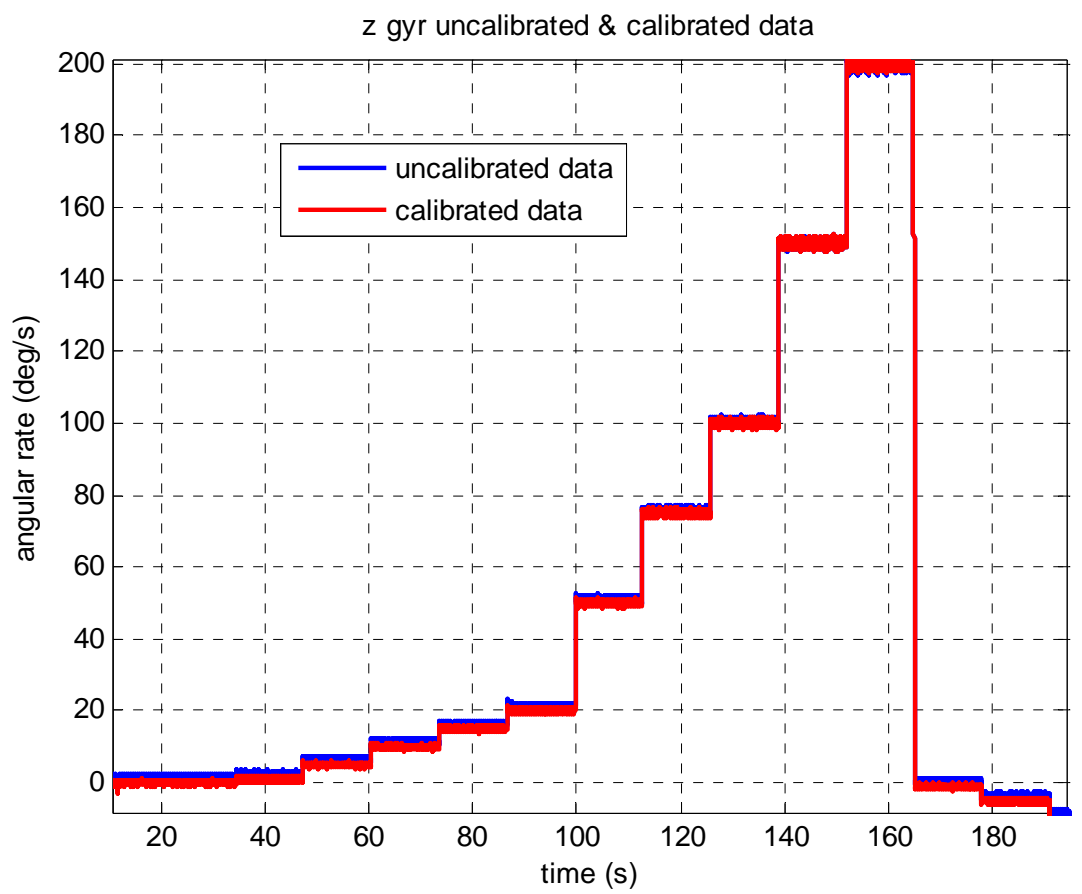

Figure 91 uncalibrated and calibrated z gyro output (real data) 
Table 16 and Table 17 summarize the total errors in the IMU sensors before and after calibration. The difference between the error levels demonstrates the importance and functionality of the error compensation model. Deterministic error estimation and compensation provided \%99 improvement in accelerometer performance. Similarly, $\% 95$ improvement was ensured in gyroscope performance.

Table 16 IMU total error before calibration

\begin{tabular}{|c|c|c|c|}
\hline & X & Y & Z \\
\hline $\begin{array}{c}\text { Gyro Error RMS } \\
\text { value }\end{array}$ & $2219.8076 \mathrm{deg} / \mathrm{h}$ & $686.9059 \mathrm{deg} / \mathrm{h}$ & $1931.243 \mathrm{deg} / \mathrm{h}$ \\
\hline $\begin{array}{c}\text { Acc Error RMS } \\
\text { value }\end{array}$ & $264.359 \mathrm{mg}$ & $204.188 \mathrm{mg}$ & $393.844 \mathrm{mg}$ \\
\hline
\end{tabular}

Table 17 IMU total error after calibration

\begin{tabular}{|c|c|c|c|}
\hline & X & Y & Z \\
\hline $\begin{array}{c}\text { Gyro Error RMS } \\
\text { value }\end{array}$ & $131.4674 \mathrm{deg} / \mathrm{h}$ & $31.8297 \mathrm{deg} / \mathrm{h}$ & $106.5706 \mathrm{deg} / \mathrm{h}$ \\
\hline $\begin{array}{c}\text { Acc Error RMS } \\
\text { value }\end{array}$ & $0.7912 \mathrm{mg}$ & $0.8465 \mathrm{mg}$ & $1.2005 \mathrm{mg}$ \\
\hline $\begin{array}{c}\text { Gyro Error } \\
\text { Repeatability }\end{array}$ & $122.2887 \mathrm{deg} / \mathrm{h}$ & $30.9998 \mathrm{deg} / \mathrm{h}$ & $80.0051 \mathrm{deg} / \mathrm{h}$ \\
\hline $\begin{array}{c}\text { Acc Error } \\
\text { Repeatability }\end{array}$ & $0.8046 \mathrm{mg}$ & $0.8612 \mathrm{mg}$ & $1.2219 \mathrm{mg}$ \\
\hline
\end{tabular}

After the deterministic error estimation and compensation, stochastic properties of the IMU were tested. These tests include gyroscope and accelerometer bias instability estimation. Results of these tests are given below. Stochastic properties of the real IMU sensors are the same as the simulated IMU. Stochastic properties of the simulated IMU are given in Table 13 The IMU data was collected in a fixed position for 120 seconds to perform stochastic error estimation test. 


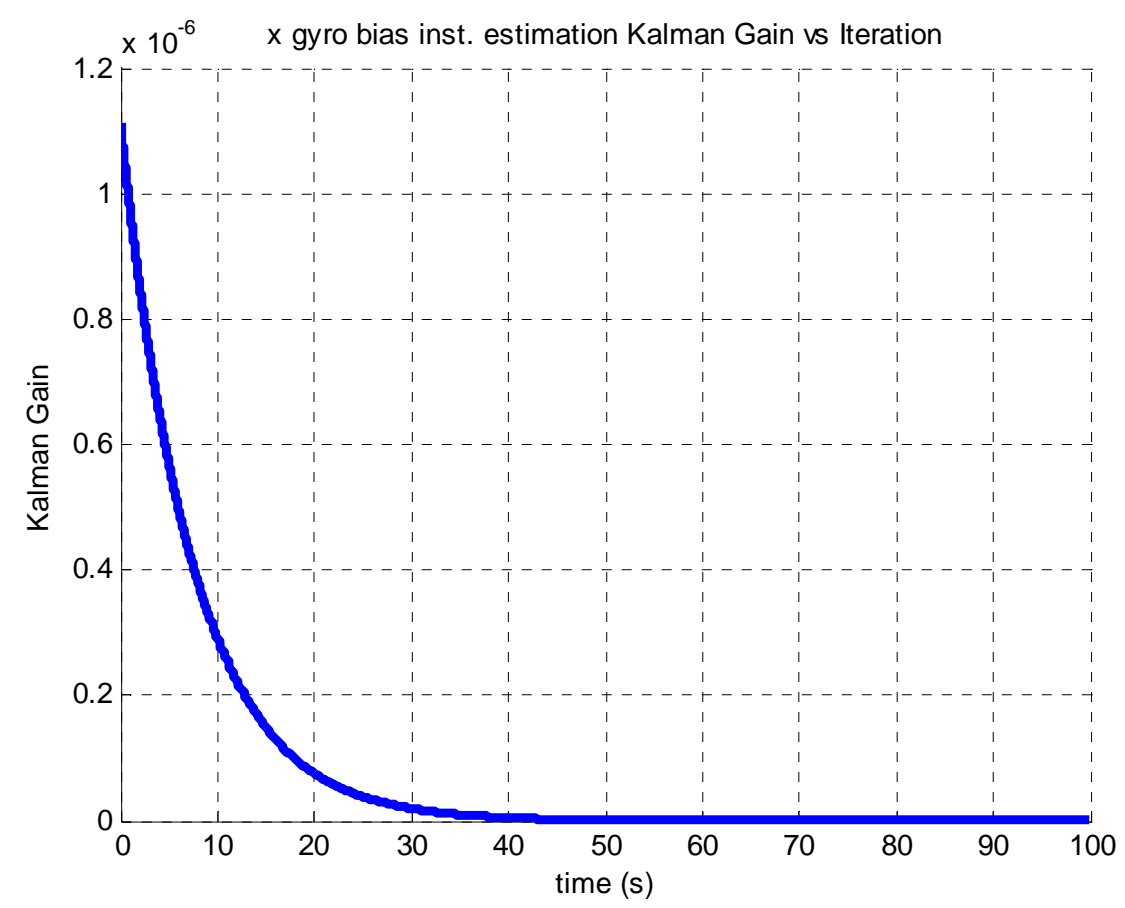

Figure 92 Gyroscope bias instability estimation Kalman gain variation (real data)

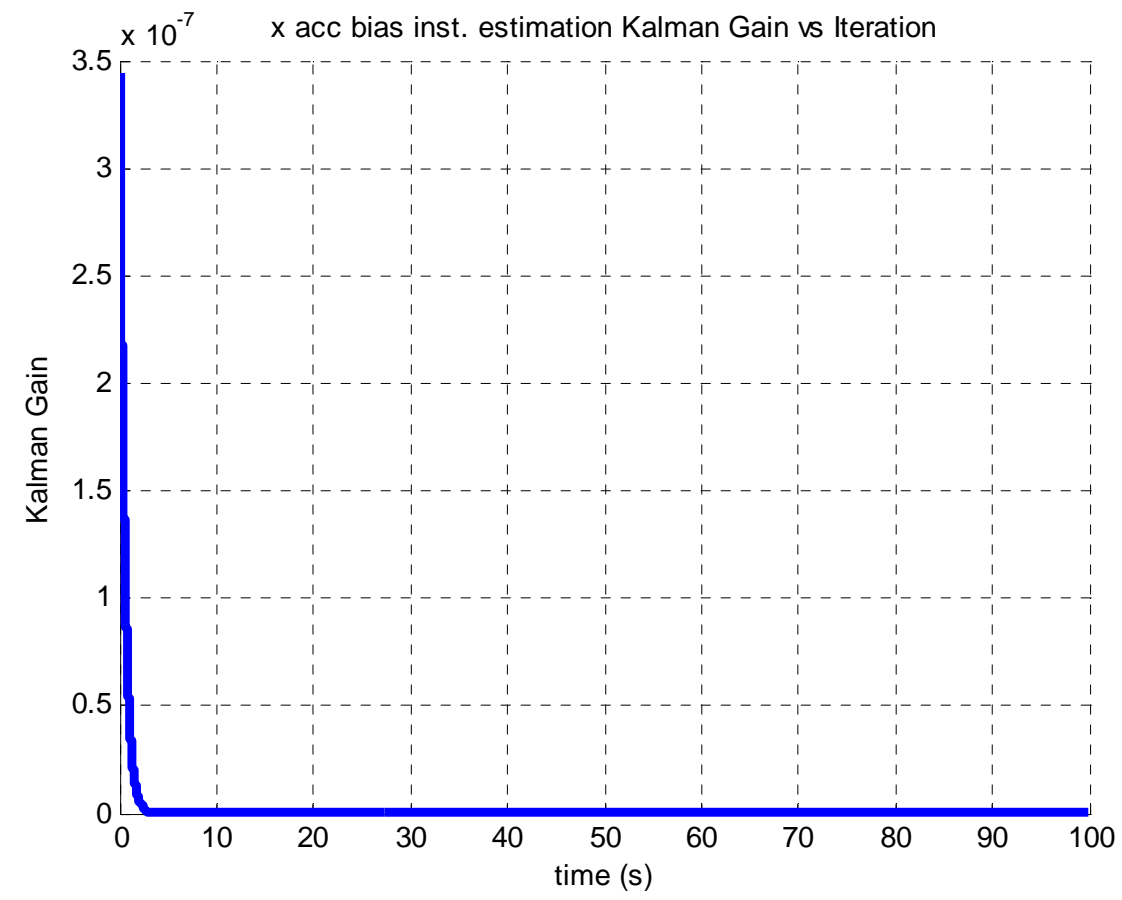

Figure 93 Accelerometer bias instability estimation Kalman gain variation (real data) 


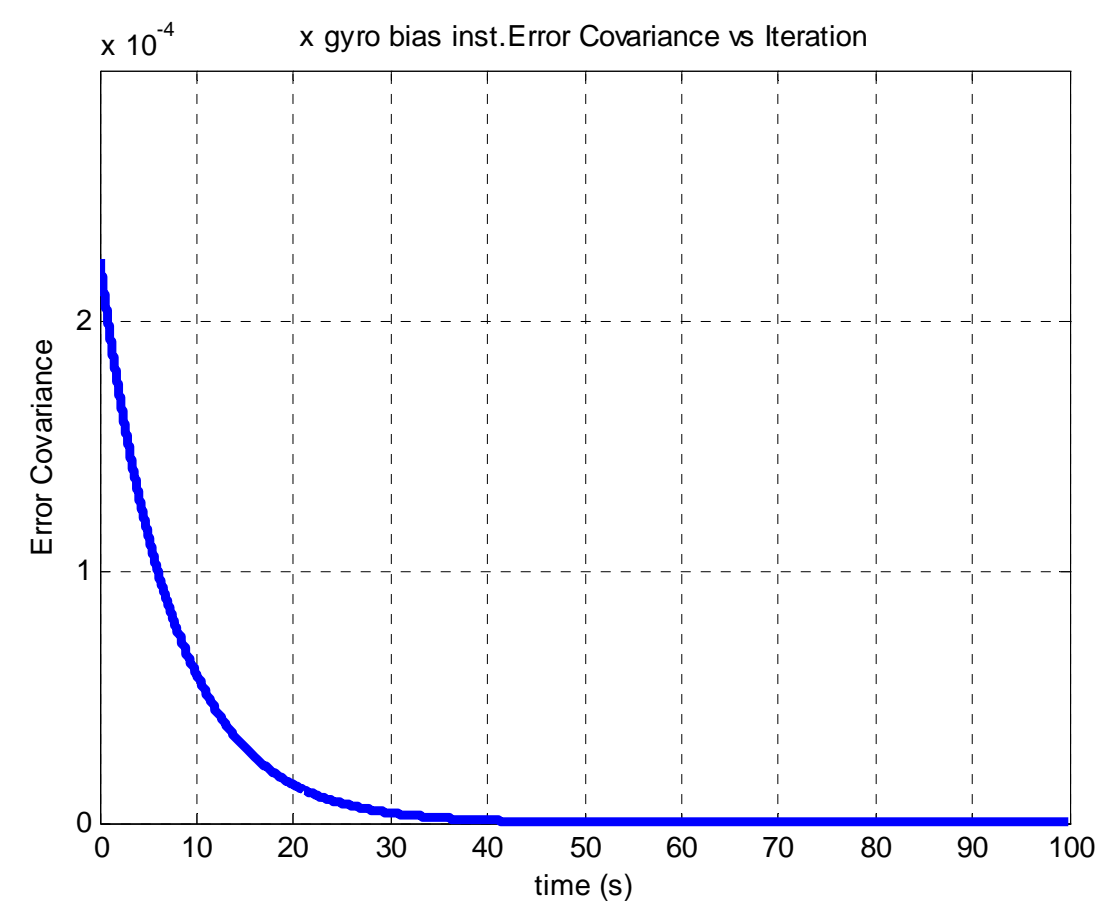

Figure 94 Gyroscope bias instability estimation error covariance variation (real data)

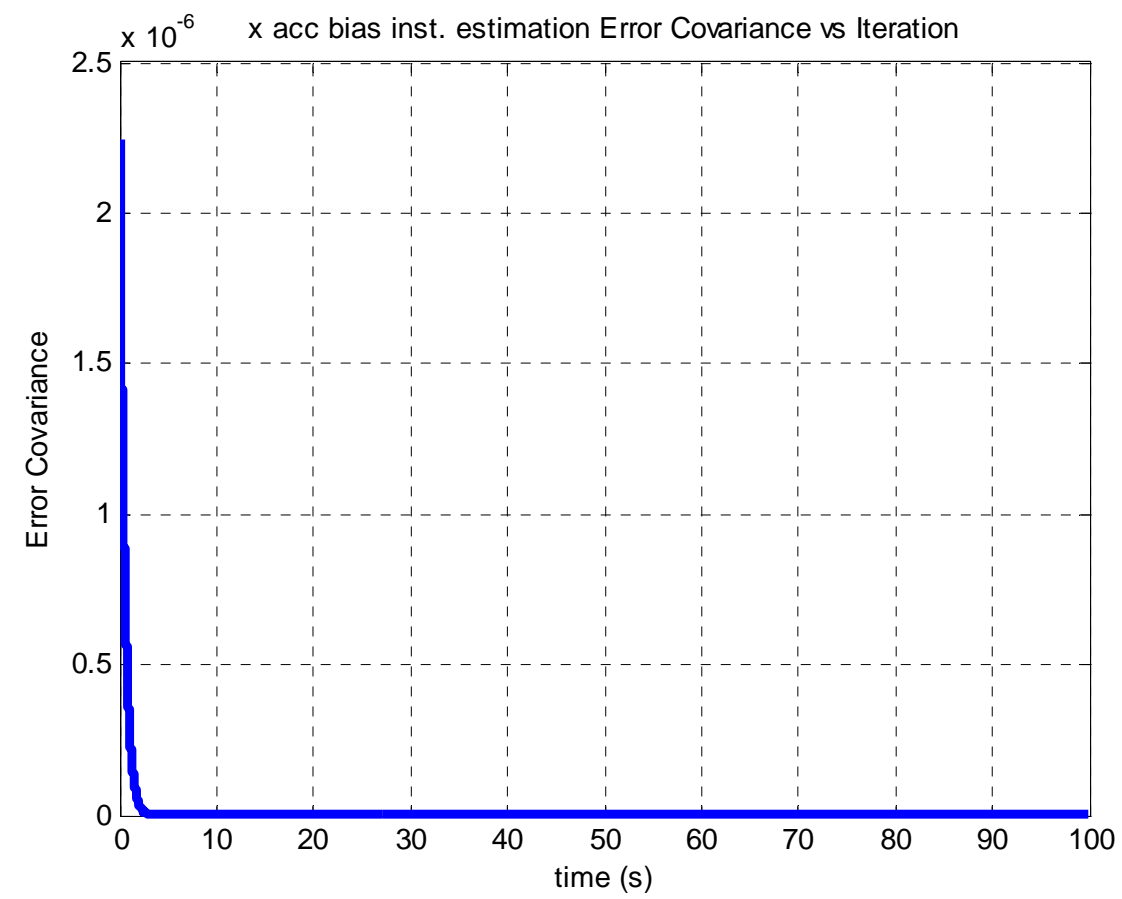

Figure 95 Accelerometer bias instability estimation error covariance variation (real data) 


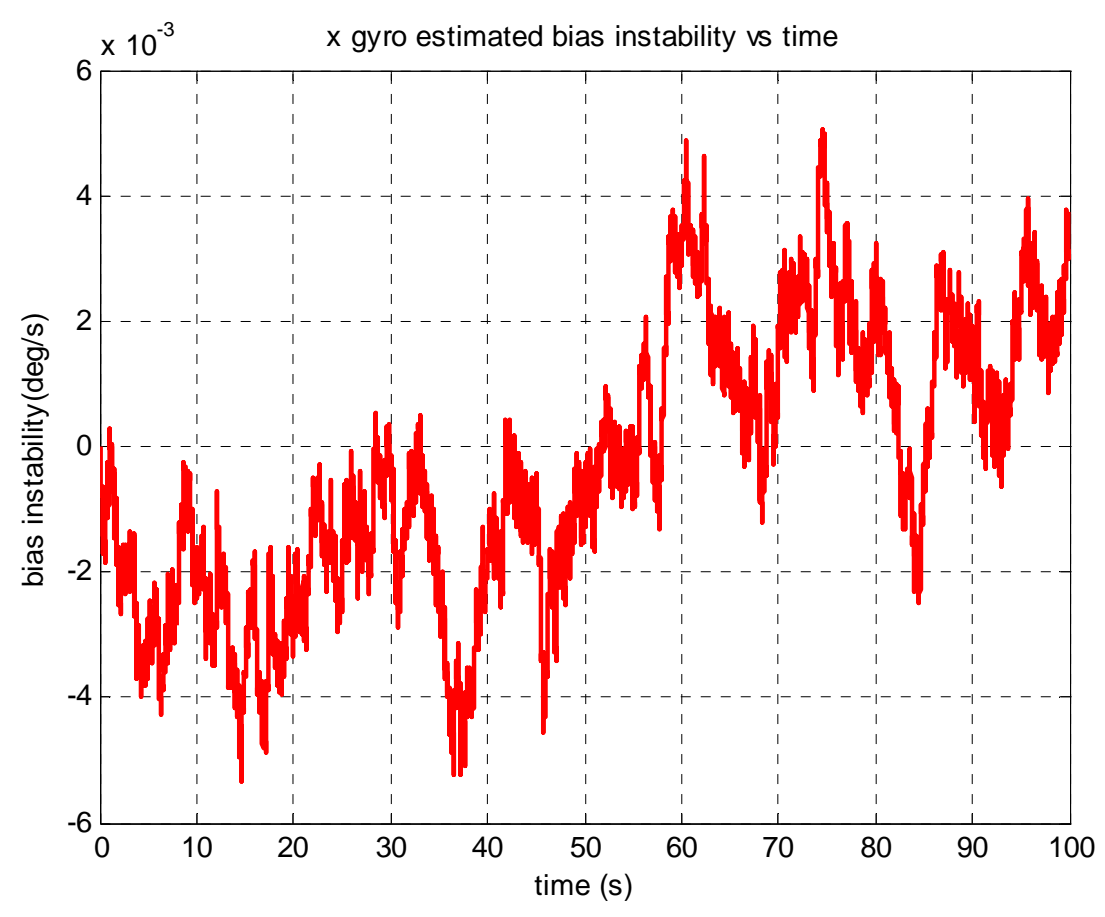

Figure $96 x$ gyro estimated bias instability (real data)

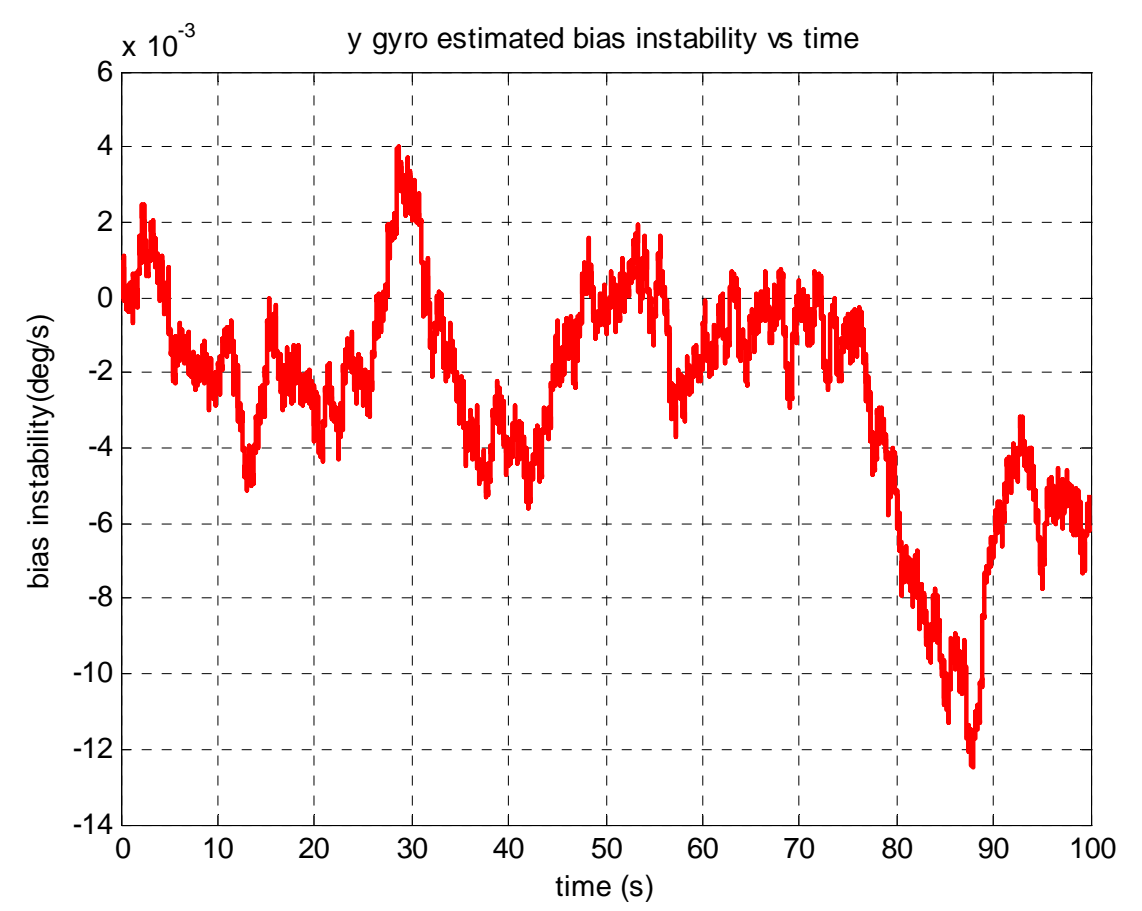

Figure 97 y gyro estimated bias instability (real data) 


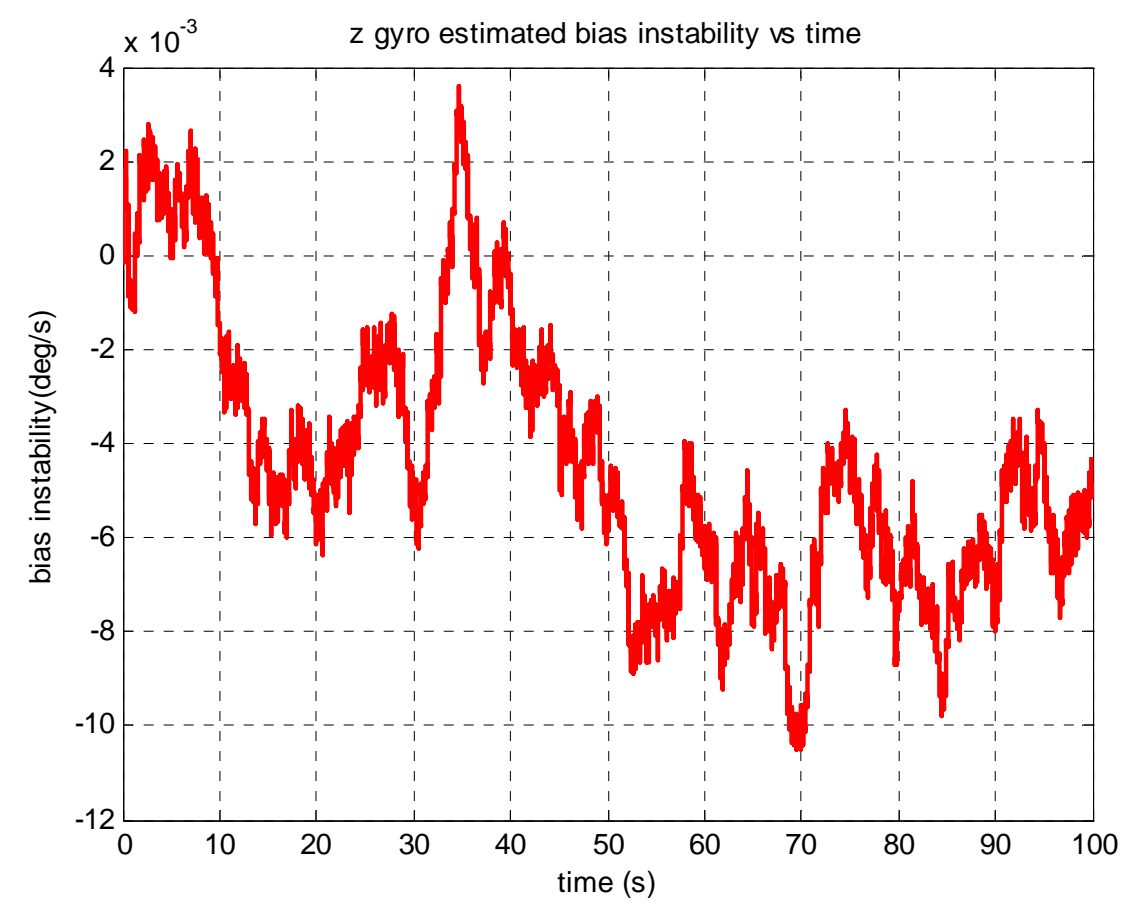

Figure 98 z gyro estimated bias instability (real data)

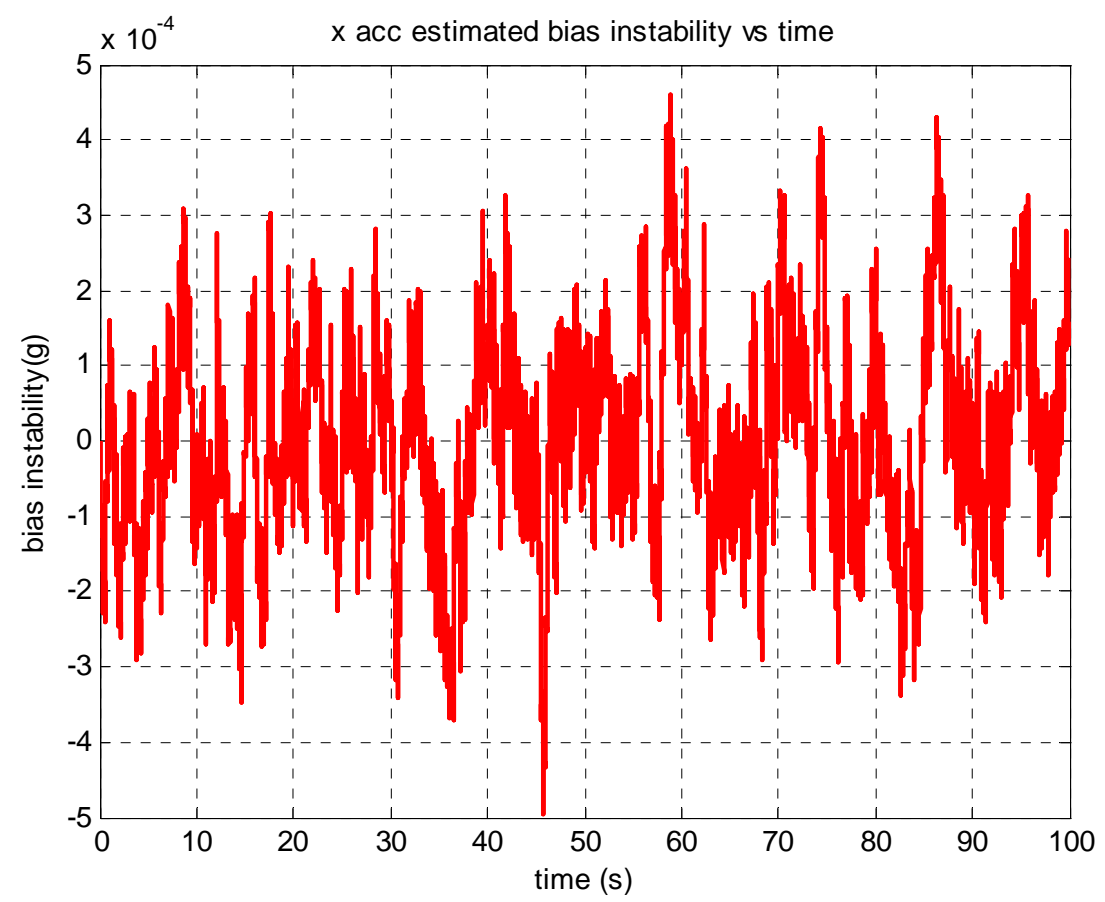

Figure $99 x$ acc estimated bias instability (real data) 


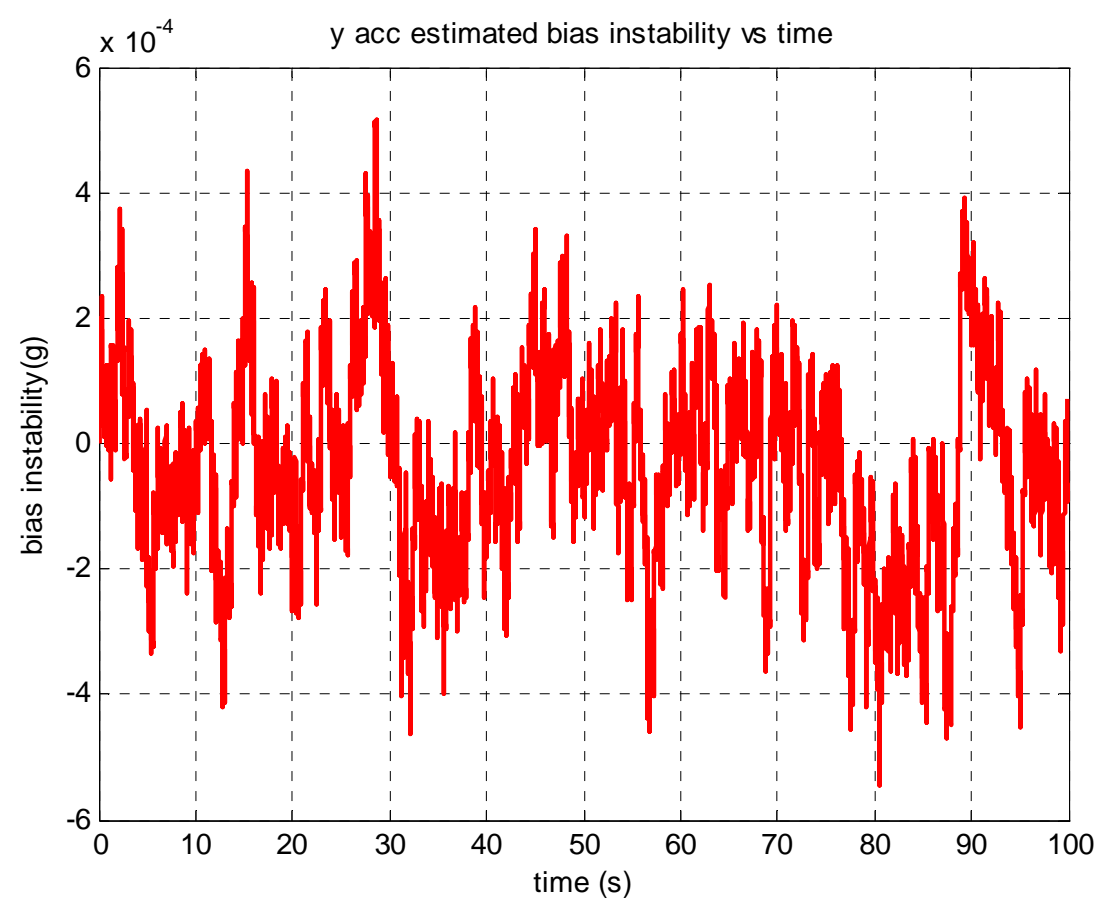

Figure 100 y acc estimated bias instability (real data)

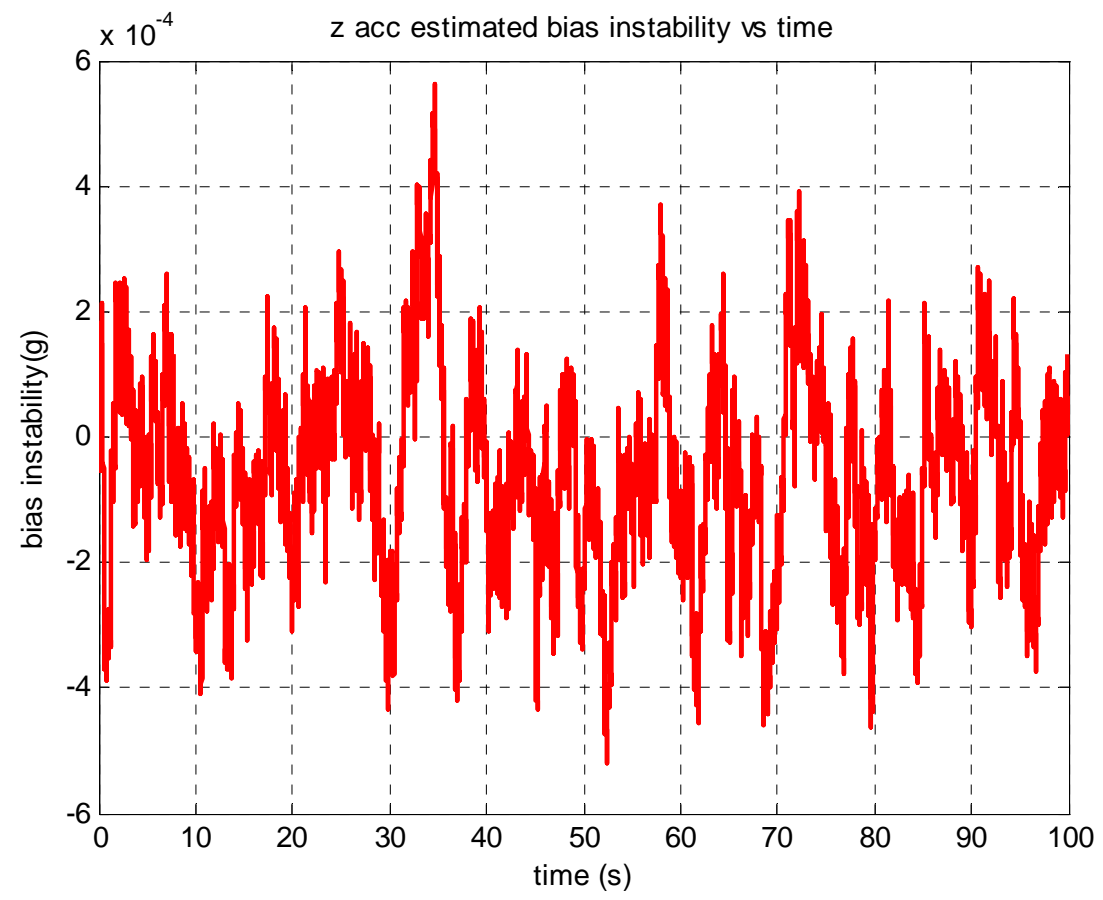

Figure 101 z acc estimated bias instability (real data) 
Stochastic error estimation algorithm was applied to experimental test data. Experimental test results are similar to simulated test results. Instantaneous values of the bias instabilities oscillates between $-3 \sigma$ and $+3 \sigma$. ( $1 \sigma$ value of the gyroscope or accelerometer bias instability is the specification of the sensor.) This results shows that performance of the estimation algorithm is consistent. Behavior of the simulation results and real experiment results are the same.

As observed from Figures 92, 93, 94 and 95 real accelerometer bias instability estimation's Kalman gain and error covariance approached before real gyroscope bias instability estimation's Kalman gain and error covariance. Correlation time and sensor noise effects can be explained as the main reason for these situations. 


\section{CHAPTER 8}

\section{CONCLUSIONS AND FUTURE WORK}

\subsection{Conclusions}

This thesis study suggests some methods for estimation of deterministic and stochastic IMU error parameters. Estimation of deterministic error parameters and stochastic error parameters were investigated separately. Firstly deterministic error estimation methods were researched. In order to determine the optimum calibration method, six position direct method and multi rate \& position method were implemented and test results were compared. According to the results, multi rate \& position method determined as the optimum method for estimation of deterministic IMU errors. After estimation of deterministic error parameters, error compensation model was designed to fix deterministic error effects. After the correction of deterministic errors, the remaining errors, difference between reference inputs and error compensation model outputs, were defined as stochastic errors and these errors were divided into two groups. First group was defined as random noise and the second group was determined as gyroscope/accelerometer bias instability. The second group errors, gyroscope and bias instabilities, were estimated by using Kalman filter algorithm. Thereby effect of bias sensor instabilities of could be corrected. The purpose of all these studies is improving the performance of MEMS IMU.

Deterministic errors are the major part of IMU errors. Therefore, deterministic error estimation and compensation algorithm design is more critical than stochastic error estimation and compensation algorithm. However, bias instability error 
compensation becomes very crucial in long-term navigation. Because of this, deterministic and stochastic error compensation should be considered together.

\subsection{Future Work}

Although, the deterministic and stochastic error compensation algorithms were developed, these algorithms are not ready to run on the processor. These algorithms must be converted to embedded code. Moreover, temperature effects on the MEMS sensors were not modeled.

As a future work, the existing algorithm can be converted to embedded code. Thereby, performance limit of the MEMS based tactical grade IMUs can be increased. Furthermore, temperature effects can be modeled and temperature effect compensation algorithm can be added to present algorithms. Besides, scale factor instability effect can be modeled and estimated by using nonlinear estimation techniques. 


\section{REFERENCES}

[1] http://www.boatsafe.com, Last access date: 25/10/2011

[2] Woodman O. J., An Introduction to Inertial Navigation, University of Cambridge, 2007

[3] D.H. Titterton, J.L. Weston, Strapdown Inertial Navigation Technology, American Institute of Aeronautics and Astronautics, 2004

[4] Averil B. Chatfield, Fundamentals of High Accuracy Inertial Navigation, American Institute of Aeronautics and Astronautics, Inc., 1997

[5] Vonk D, Inertial Measurement Unit, University of New South Wales

[6] Aggarwal, P., Syed Z., El-Sheimy N., Noureldin A. MEMS Based Inertial Navigation, 2010,

[7] NATO RTO-SET-054/RTG-30 Panel, Basic Guide to Advanced Navigation, 2003

[8] Lawrence, A., Modern Inertial Technology, Springer,1998

[9] George T. Schmidt, “INS/GPS Technology Trends” NATO RTO-SET-116 Panel, 2010

[10] Roylance L.M., Angell J.A., " A batch-fabricated silicon accelerometer" IEEE Trans. Electron Devices, vol. ED-26, pp. 1911-1917, Dec. 1979

[11] Barbour, N., et al, "Micromachined Inertial Sensors for Vehicles", IEEE Conference on Intelligent Transportation System, pp. 1058-1063,1997

[12] Kamen E.W., Su J.K., Introduction to Optimal Estimation, Springer, 1999

[13] Lewis L.F., Lihua X., Popa D., Optimal and Robust Estimation, CRC Press, 2008

[14] Welch,G., Bishop G., “An Introduction to the Kalman Filter”, 2006

[15] Groves, P.D., Principles of GNSS, Inertial , and Multisensor Integrated Navigation Systems, Artech House, 2008

[16] Marvel, D., Integration of the Global Positioning System with an INS, , 1998 
[17] Nassar, S., “Accurate INS/DGPS positioning using INS data de-noising and autoregressive model of inertial sensor errors", Geomatica 59 (3), pp.283294,2005

[18] Colibrys MS9000.D Series Capacitive Accelerometer Datasheet, 2011

[19] Kayasal, U., Modeling and Simulation of Navigation System with an IMU and a Magnetometer, METU Thesis, 2007

[20] Flenniken, W., Wall J., Bevly D.M., "Characterization of Various IMU Error Sources and Effect on Navigation Performance", Proceedings of ION GNSS, 2005

[21] El-Diasty, M., Pagiatakis S., "Calibration and Stochastic Modelling of Inertial Navigation Sensor"

[22] Ruben, E., Gyroscopes, Mechatronics Literature Survey, Utah State University, 2009

[23] http://en.wikipedia.org/wiki/Vibrating_structure_gyroscope, Last access date: $23 / 12 / 2011$

[24] Ünlüsoy, Ö., Reliability Analysis Process And Reliability Improvement of An Inertial Measurement Unit, METU Thesis, 2010

[25] Aslan, G., Saranlı, A. "Characterization and Calibration of MEMS Inertial Measurement Units"

[26] IEEE Std 1293-1998 Standard Specification Format Guide and Test Procedure for Linear Single Axis, Nongyroscopic Accelerometers

[27] IEEE Std 1431 ${ }^{\mathrm{TM}}-2004$ Standard Specification Format Guide and Test Procedure for Coriolis Vibratory Gyros 


\section{APPENDIX}

\section{FLOWCHART OF THE SIMULATION AND EXPERIMENTAL STUDIES}

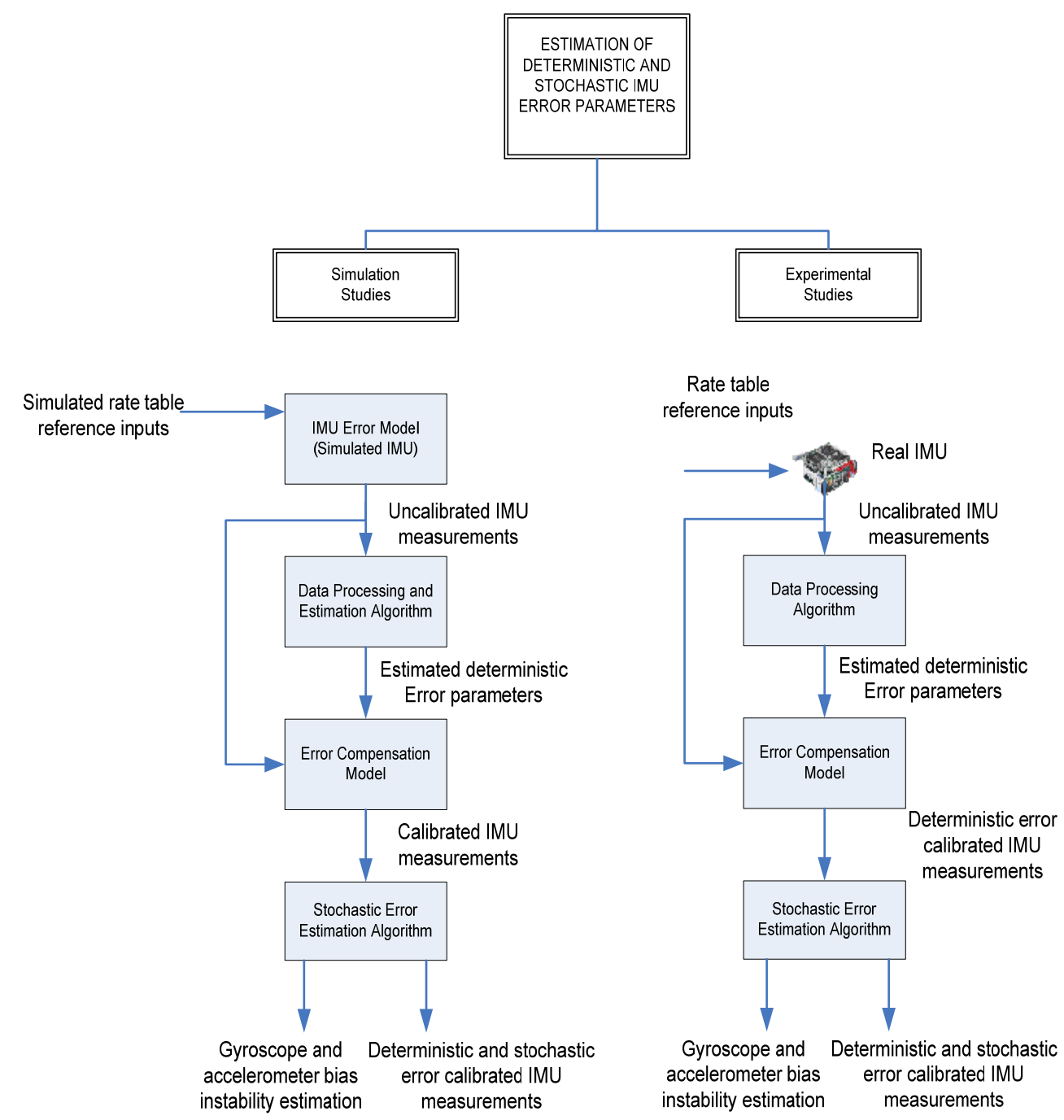

